\title{
Activities promoting personal health potentials - Development of a priority concept for German general practi- tioners' surgeries
}

Doctorate in Business Administration

\begin{tabular}{|l|l|}
\hline Student name: & Ballin, Cedric \\
\hline Student number: & S1111797 \\
\hline Cohort: & Munich, 6 \\
\hline Advisor: & Dr. Ivanna Adamson \\
& Ar. Francisca Veale \\
\hline Submitted & $\begin{array}{l}\text { A thesis submitted to the University of Gloucestershire in } \\
\text { accordance with the requirements of the degree of Doctor- } \\
\text { ate in Business Administration in the Faculty of Business'; }\end{array}$ \\
\hline
\end{tabular}




\section{Content}

$\begin{array}{lr}\text { List of tables } & 8\end{array}$

$\begin{array}{ll}\text { List of figures } & 9\end{array}$

$\begin{array}{ll}\text { 1. Abstract } & 11\end{array}$

$\begin{array}{lr}\text { 2. Introduction } & 13\end{array}$

$\begin{array}{lr}\text { 2.1 Contextual Background } & \mathbf{1 4}\end{array}$

2.1.1 Area 1: General health care management .................................................. 14

2.1.2 Area 2: Corporate health management...................................................... 15

2.1.3 Area 3: Business performance and quality in health management .............. 16

2.1.4 Area 4: Diseases and disease prevention at the workplace .......................... 16

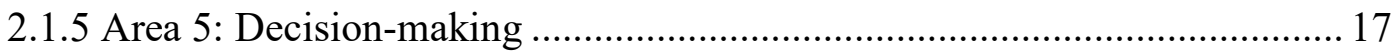

2.2 German general practitioners' surgeries and health management 19

2.2.1 The structure and size of German general practitioners' surgeries.............. 20

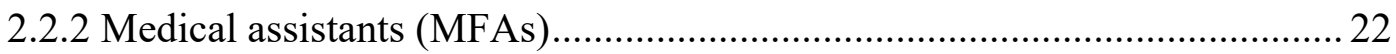

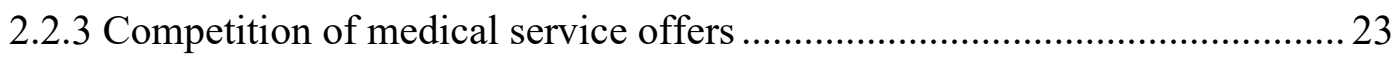

2.2.4 Area 1: General health care management ................................................. 23

2.2.5 Area 2: Corporate health management.................................................... 24

2.2.6 Area 3: Business performance and quality management ............................. 26

2.2.7 Area 4: Diseases and disease prevention at the workplace ......................... 28

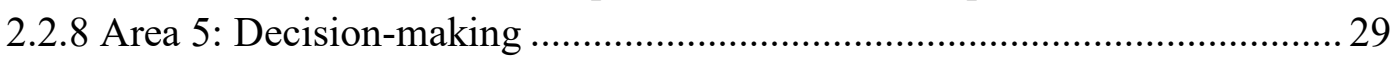

2.3 Research questions and the way to answer them 31

2.4 Overview: The chapters of this research 31

2.5 Summary 33

3. Literature review 35

3.1 Models and activities to reduce absenteeism 36

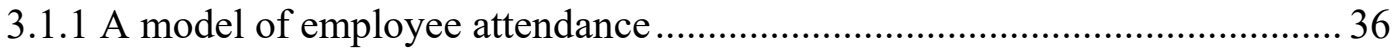

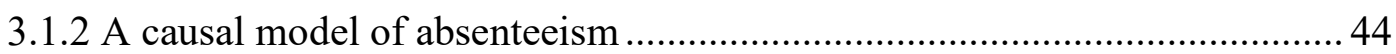

3.1.3 Peer reviewed activities promoting personal health potentials .................... 50

3.2 A framework to increase performance in micro-enterprises 59

3.2.1 Resource-based approaches for micro-enterprises ....................................6 60

3.2.2 Psychological contract and the employee relationship in SMEs ................. 61

3.2.3 The link between HR practices, psychological contract and performance ... 65 


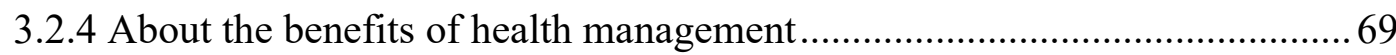

3.3 Bridging the implementation gap $\quad \mathbf{7 1}$

3.3.1 The implementation process and the psychological contract...................... 72

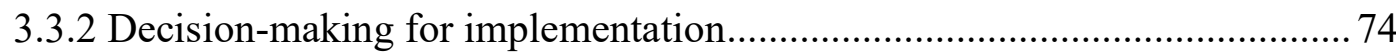

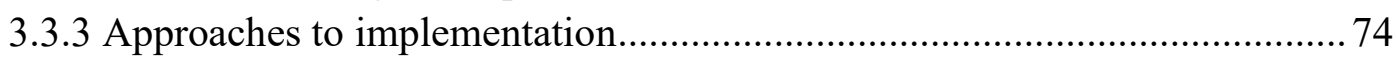

3.3.4 Deriving priorities for German general practitioners' surgeries .................. 77

$\begin{array}{lr}\text { 3.4 Summary } & 87\end{array}$

$\begin{array}{lr}\text { 4. Methodology } & 89\end{array}$

4.1 Research paradigm and research philosophy 89

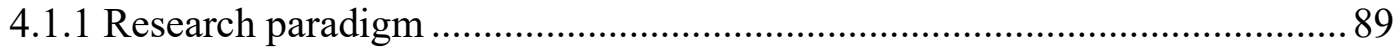

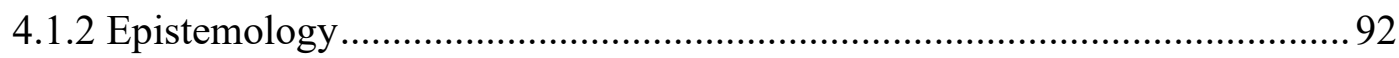

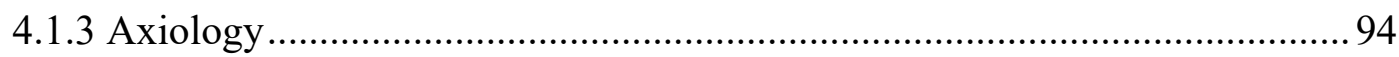

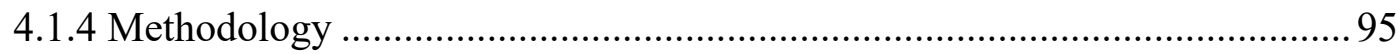

4.2 Methods for questionnaire design $\quad 96$

4.2.1 Questions about demographic characteristics .......................................... 98

4.2.2 Questions about the general practitioner's surgery ................................... 99

4.2.3 Questions about the promotion of personal health potentials .................... 100

4.2.4 Checking the usability of the questionnaire ............................................... 106

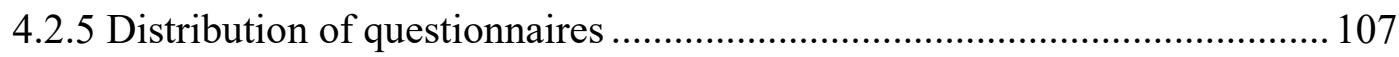

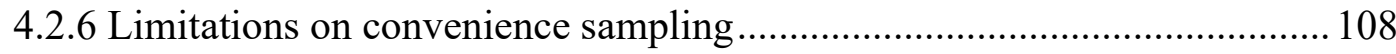

4.2.7 Hypotheses on the results of the MFA survey ....................................... 110

4.3 Methods for the development of a priority concept 113

4.3.1 Methods to solve ranking problems - problem structuring....................... 113

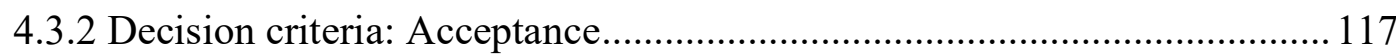

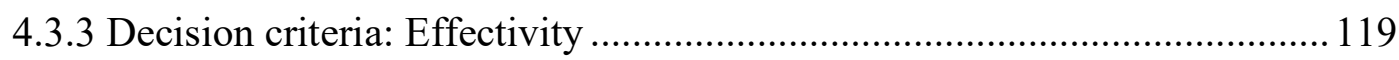

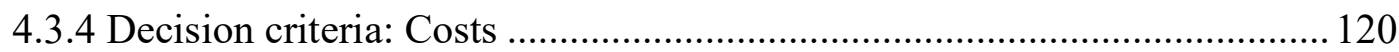

4.3.5 Software application: Parameters for M-MACBETH.............................. 122

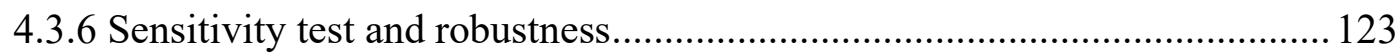

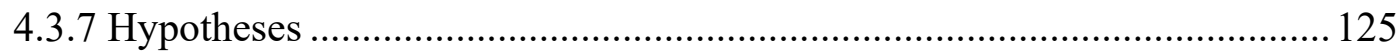

4.3.8 Methods for evaluation of implementation aspects................................. 126

$\begin{array}{ll}\text { 4.4 Ethical considerations } & 127\end{array}$

4.4.1 Data protection and protection of the participants ................................... 127

4.4.2 Anonymity and possible ethical conflicts .............................................. 129

4.4.3 Role of the researcher and ethical considerations .................................... 129

$\begin{array}{ll}\text { 4.5 Summary } & 132\end{array}$

5. Research findings of the MFA survey $\quad 134$ 
5.1 Quantitative and qualitative data from the MFA survey 134

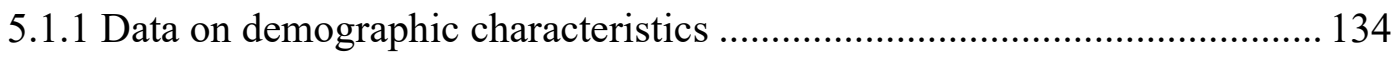

5.1.2 Data on the general practitioner's surgery ............................................. 135

5.1.3 Data on the promotion of personal health potentials ................................ 137

5.2 Preferences of the MFAs on health-promoting measures 143

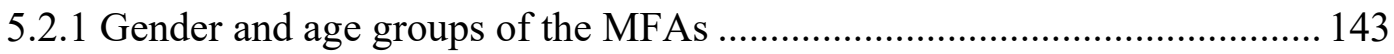

5.2.2 Experience with and willingness for health management.......................... 146

5.2.3 Preference profile to programs of movement promotion .......................... 151

5.2.4 Preference profile to programs of nutrition counselling ........................... 155

5.2.5 Preference profile to programs of vaccination .......................................... 160

5.2.6 Acceptance of further health management activities ................................ 162

5.2.7 Preference profiles to combined programs ............................................. 164

$\begin{array}{lr}\text { 5.3 Summary of results } & 167\end{array}$

6. Research findings for the development of a priority concept 170

$\begin{array}{ll}\text { 6.1 Scoring the acceptance } & 171\end{array}$

$\begin{array}{lr}\text { 6.2 Scoring the effectivity } & 172\end{array}$

$\begin{array}{lr}\text { 6.3 Scoring the cost level } & 176\end{array}$

$\begin{array}{lr}\text { 6.4 Generating a priority list } & 178\end{array}$

$\begin{array}{lr}\text { 6.5 Sensitivity and robustness test } & 181\end{array}$

6.5.1 Sensitivity versus changes in the acceptance weighting ......................... 181

6.5.2 Sensitivity versus changes in the effectivity weighting ........................... 183

6.5.3 Sensitivity versus changes in the cost level weighting ............................. 185

$\begin{array}{ll}\text { 6.6 Hypotheses on scoring } & 186\end{array}$

$\begin{array}{lr}\text { 6.7 Summary of results } & 188\end{array}$

$\begin{array}{ll}\text { 7. Discussion } & 191\end{array}$

7.1 Results of structured interviews with general practitioners 191

7.1.1 Characteristics of the interviewed general practitioners' surgeries ............ 192

7.1.2 Comparison with the priority list .......................................................... 193

7.1.3 Factors influencing productivity and performance .................................... 195

7.1.4 Implementation of health management ................................................. 196

$\begin{array}{ll}\text { 7.2 Implementation of the standard proposal } & 197\end{array}$

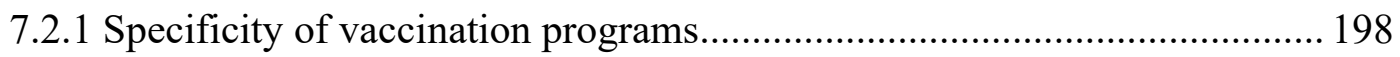

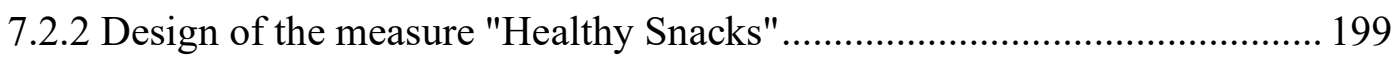


7.2.3 Design of the measure "Training at Home" ................................................ 200

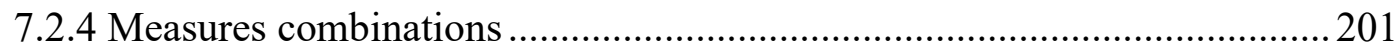

7.3 Adaptations of the standard proposal $\quad 202$

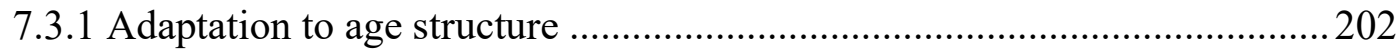

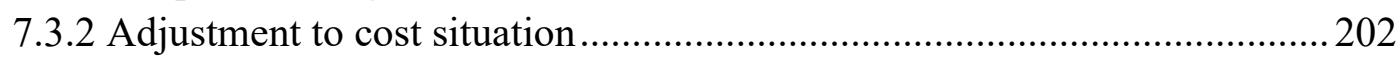

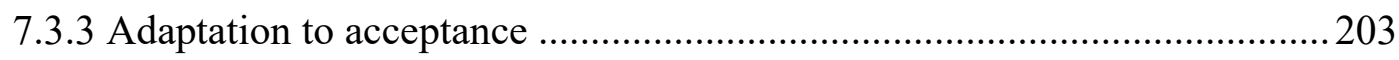

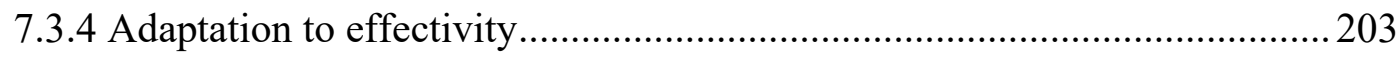

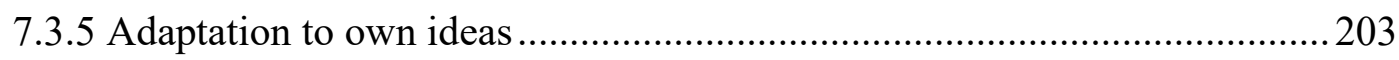

7.4 Development of a project "Health Management System" 204

7.4.1 Target system and leadership ability .................................................... 205

7.4.2 Further measures of primary prevention .................................................206

7.4.3 Further measures to reduce risk factors....................................................2. 208

7.5 Integration of the proposed concept into quality management 209

$\begin{array}{ll}\text { 7.6 Bridging the implementation gap } & \mathbf{2 1 1}\end{array}$

7.6.1 The relevance of primary prevention for MFAs …...................................2 212

7.6.2 Content-oriented and process-oriented perspectives on implementation.... 213

7.6.3 The psychological contract and leadership ability ................................ 214

$\begin{array}{ll}\text { 7.7 Summary of results } & 216\end{array}$

$\begin{array}{lr}\text { 8. Conclusions } & 218\end{array}$

$\begin{array}{ll}\text { 8.1 Contribution to empirical research } & \mathbf{2 1 8}\end{array}$

8.1.1 Health management in general practitioners' surgeries ........................... 219

8.1.2 Human resource management ......................................................... 220

8.1.3 Small-, medium-, and micro-enterprises .............................................. 222

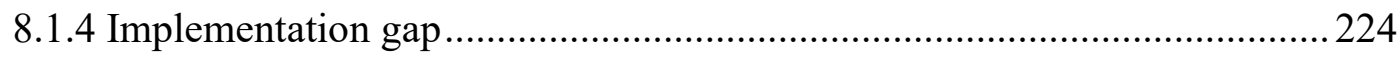

$\begin{array}{ll}\text { 8.2 Contribution to methodology } & 225\end{array}$

$\begin{array}{ll}\text { 8.3 Contribution to practice } & \mathbf{2 2 7}\end{array}$

8.3.1 Adaptation to individual stakeholder realities............................................229

8.3.2 Specializations of medical practices with MFAs ..................................... 229

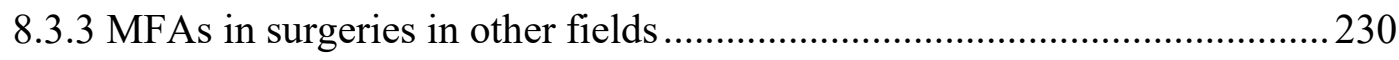

$\begin{array}{ll}\text { 8.4 Limitations of the research } & 231\end{array}$

$\begin{array}{ll}\text { 8.5 Future research } & 232\end{array}$

$\begin{array}{ll}\text { 8.6 Summary } & 233\end{array}$

$\begin{array}{lr}\text { 9. References } & 235\end{array}$ 
10. Appendices

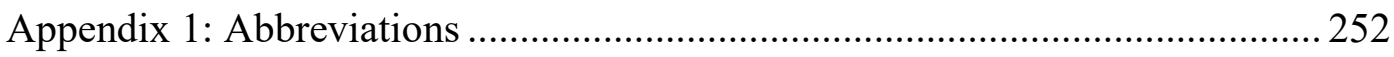

Appendix 2: Results of literature research "Personal health potentials" ............. 256

Appendix 3: Original (German) questionnaire ............................................... 259

Appendix 4: Results of the questionnaires..................................................... 261

Appendix 5: The structured interview guide for the general practitioners .......... 264

Appendix 6: Documentation of the answers in the interview guide ................... 269

Appendix 7: Data collection sheets for scoring results .................................... 283 


\section{List of tables}

Table 1: Absent days of German employees (Grobe, Dörning et al., 2011) ........................... 51

Table 2: Cross-impact matrix of the interaction of health management goals......................... 85

Table 3: The list of proposed measures to be prioritized ................................................. 117

Table 4: Scoring the acceptance of personal health promotion activities ............................... 118

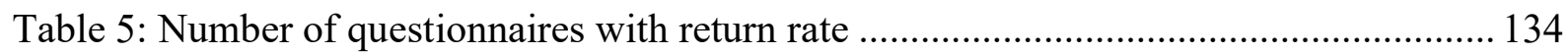

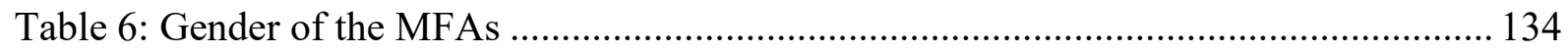

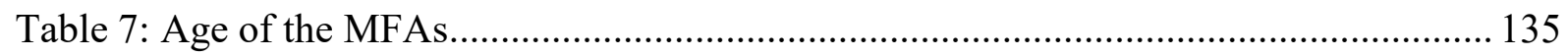

Table 8: Specialization of the doctor's office, in which the MFAs work ................................. 135

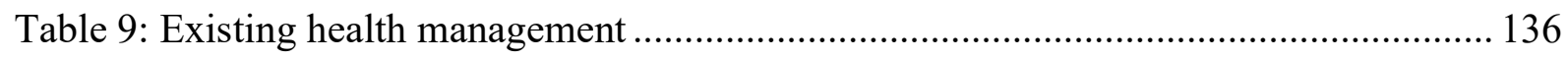

Table 10: Existing health management activities for the open question "yes"...................... 136

Table 11: Possible reasons for lack of health management for open question "no" .............. 137

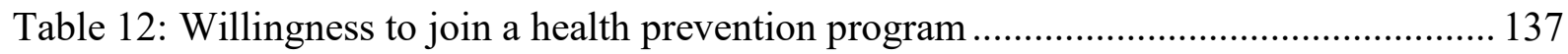

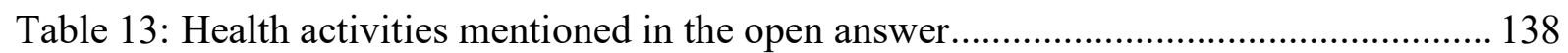

Table 14: Reasons for refusal of health activities in the open answer .................................. 138

Table 15: Choice of "movement promotion" programs total ticks ....................................... 139

Table 16: Choice of "movement promotion" programs in percentages................................... 139

Table 17: Choice of "movement promotion" programs reduced to "yes" or "no" ................ 139

Table 18: Choice of "Weight reduction and change of nutrition" - total ticks ....................... 140

Table 19: Choice of "Weight reduction and change of nutrition" - percentages .................... 140

Table 20: Choice of "Weight reduction and change of nutrition" program - reduced

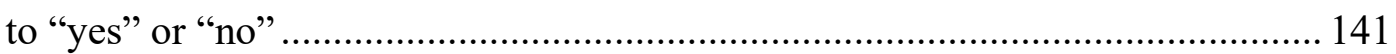

Table 21: Quantitative data on the use of vaccines in private life ....................................... 141

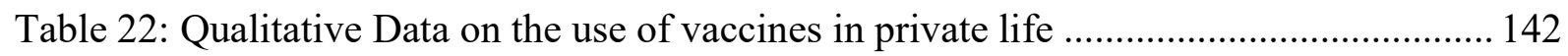

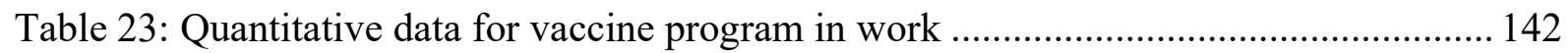

Table 24: Qualitative data for a vaccine program in work.................................................. 143

Table 25: Quantitative data on vaccines status check ......................................................... 143

Table 26: Hypothesis test: Willingness to participate is independent of age ......................... 148

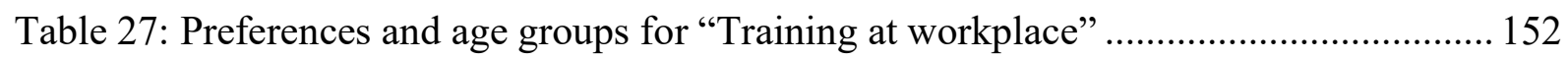

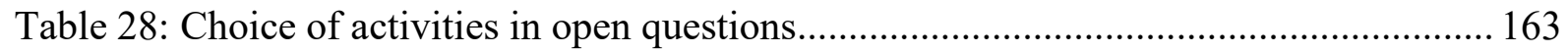

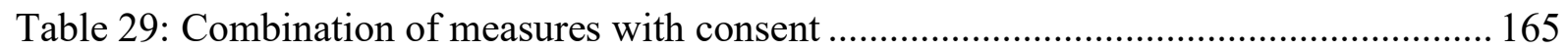

Table 30: Scoring the acceptance of personal health promotion activities ............................ 172

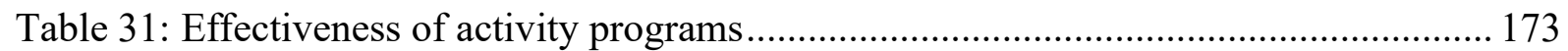

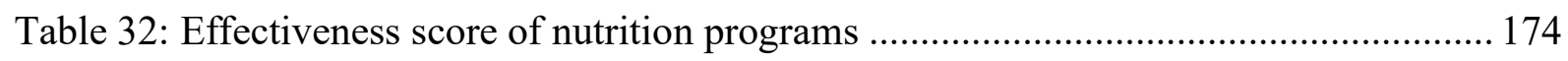

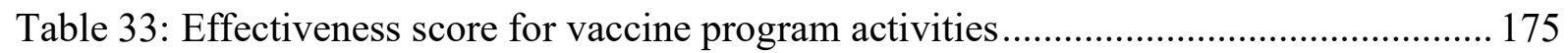

Table 34: Score of the effectivity of personal health promotion activities ............................ 175

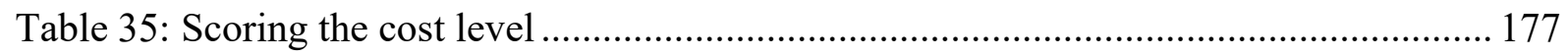

Table 36: Building the score of the costs of personal health promotion activities ................ 177

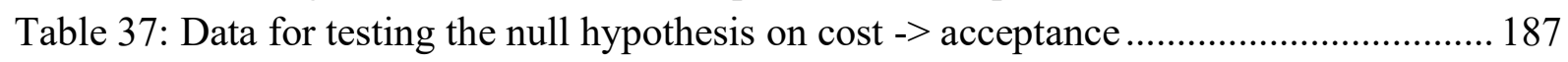

Table 38: Importance of levers for reducing absent days - extracted from the interviews with the general practitioners ........................................................ 196 


\section{List of figures}

Figure 1: The salutogenetic conception - source: Hertel, 1992 cited in Helmenstein et

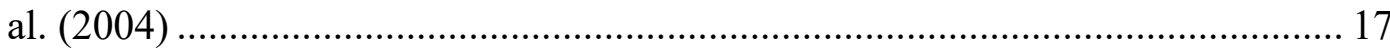

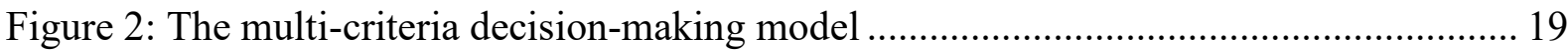

Figure 3: Self-assessment of the consultancy competence of the general practitioners in the most important primary prevention areas - source: (Regus, 2012) ............. 29

Figure 4: A model of employee attendance (Rhodes and Steers, 1978, p. 53) ....................... 37

Figure 5: Causal model of absenteeism and psychological contract.................................... 45

Figure 6: Literature search as starting point of the multi-criteria decision-making

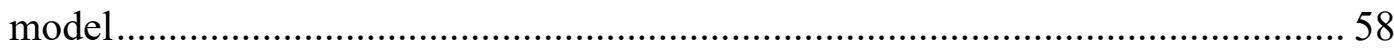

Figure 7: An HR practices - psychological contract - organizational linkage framework (Katou, 2013, p. 572 combined with p. 583)

Figure 8: The job design of MFAs fits to the vocational training (source:

Bundesinstitut für Berufsbildung BIBB, 2012 - translated by the author) 80

Figure 9: Assessment of the delegation of preventive advice to MFAs with absolute and relative frequencies; According to the frequency of the assessments "clearly sensible" and "rather sensible" - (Regus, 2012)

Figure 10: Fourfold table for identifying priorities in the control levers .............................. 85

Figure 11: Questionnaire design as part of the multi-criteria decision-making model........... 97

Figure 12: Weighting as part of the multi-criteria decision-making model......................... 114

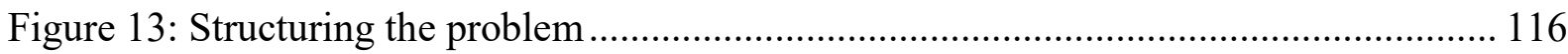

Figure 14: Weighting criteria and consistent judgements (Screenshots M-

MACBETH)

Figure 15: Options and activities (Screenshot M-MACBETH) ......................................... 123

Figure 16: Sensibility test in the multi-criteria decision-making model.............................. 124

Figure 17: Example of a sensibility analysis (Screenshot M-MACBETH) .......................... 125

Figure 18: Implementation - the last step in the multi-criteria decision-making model ........ 126

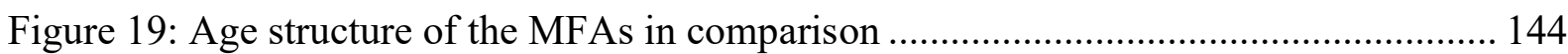

Figure 20: Different willingness to participate in health-promoting measures ..................... 149

Figure 21: Group formation with the combination "experience" and "willingness" ............. 150

Figure 22: Differing preferences between the age groups ............................................... 152

Figure 23: Preferences for the different measures of movement promotion and age

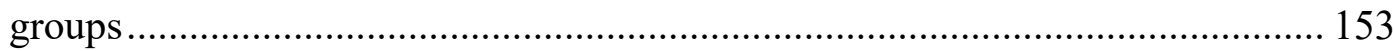

Figure 24: Evaluation of the Likert scale for all proposed activities .................................... 157

Figure 25: Preferences for the different measures of weight reduction and change of

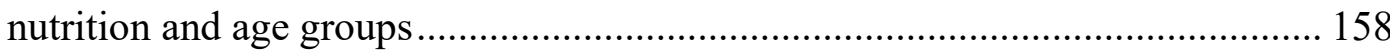

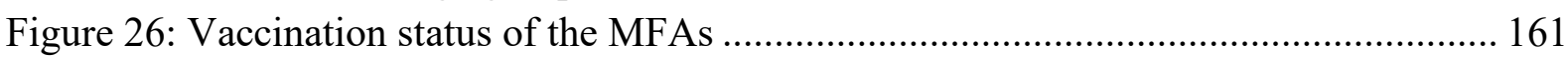

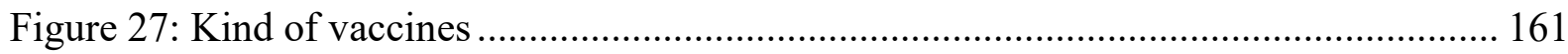

Figure 28: Approbation for measure combinations ...................................................... 165

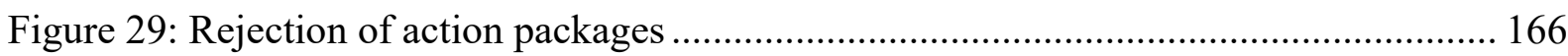

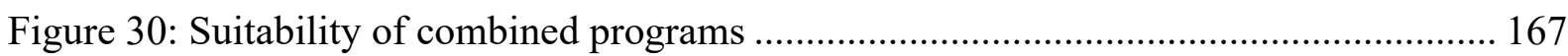

Figure 31: Multi-criteria decision-making model: Scoring the decision criteria .................... 170

Figure 32: Options and activities list (screenshot) .......................................................... 178 
Figure 33: Score as performance indicator for a certain option/activity and decision

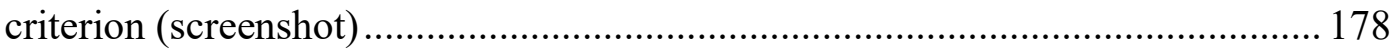

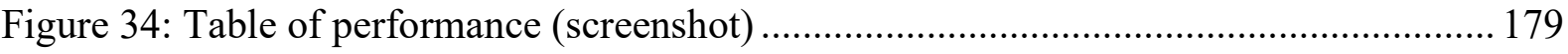

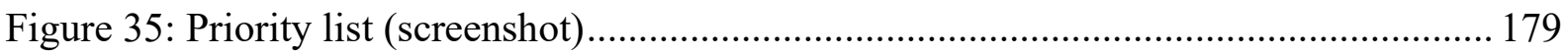

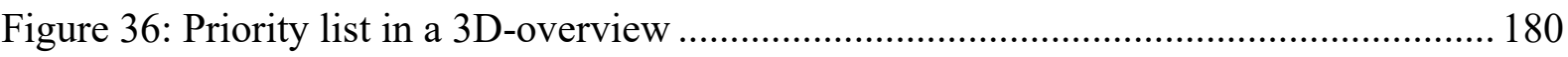

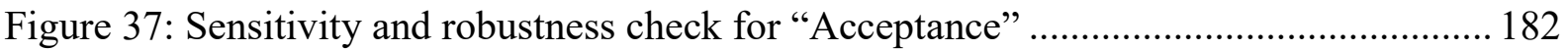

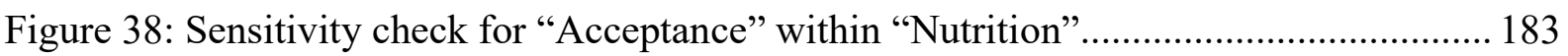

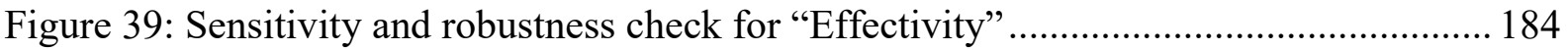

Figure 40: Sensitivity and robustness check for "Effectivity" and "Nutrition"..................... 184

Figure 41: Sensitivity and robustness check for "Costs" and "Training" ............................ 185

Figure 42: Sensitivity and robustness check for "Costs" and "Nutrition" .............................. 185

Figure 43: Scatter diagram for the correlation: Cost level - Acceptance ............................... 187

Figure 44: Scatter diagram for the correlation: Effectivity - Acceptance ............................... 188

Figure 45: The trend to healthy snacks at work (TK, 2017 - translated by the author) ......... 200

Figure 46: Operational HRM-Model for German general practitioners' surgeries ............... 223

Figure 47: The multi-criteria decision-making model for German general practitioners' surgeries 


\section{Abstract}

The focus for this research is health management in German general practitioners' surgeries. In Germany, there are over 50,000 general practitioners' surgeries with over 150,000 physician's assistants as employees. One of the main objectives of health management is the reduction of absent days due to ill health and it is important to know, which activities can be identified to reach this objective.

The overall aim of this research is to develop a concept to support stakeholders in promoting health supporting activities which facilitate personal health potentials and help improve the productivity of the general practitioners' surgeries.

The basis for the development of this concept was a systematic literature review, which indicated that health management in general could be improved by health promoting activities. However, the findings from the literature review reveal gaps in general practitioners' surgeries health promotion practices. This research aims to improve the knowledge in health management at general practitioners' surgeries in Germany with a focus on activities promoting the employees' health potentials.

The success of the proposed activities essentially depends on the acceptance by the employees. Therefore, a questionnaire about "health management" for employees of general practitioners' surgeries has been developed which used the health-promoting measures found in the literature review. The findings of these questionnaires provide quantitative data $(\mathrm{N}=194)$ on which of the health promoting activities are preferred by the employees. The findings give an insight into personal preferences for healthpromoting activities from the employee's point of view.

The findings of the employee survey were used as indicator for the acceptance of activities; the results of the systematic literature review were used as indicator for the effectiveness. As a third indicator, the costs of the discussed activities have been incorporated into the concept. These three indicators form the basis for a multi-criteria decision analysis. Results were used to build a score for generating a priority list with ten effective activities promoting personal health potentials.

The measures with the highest preferences are vaccines (94\%), healthy food at work $(67 \%)$, and training at home (61\%). Robustness of the results was tested by means of a sensitivity analysis. Vaccination is widely ranked first in the priority list. This is of importance as the employees of general practitioner's surgeries are exposed to substantial risk of infection.

Three different strategies for implementing this concept into health management in general practitioners' surgeries were developed.

The main contribution to knowledge is seen in the contribution to the methodology. It shows how different perspectives of different stakeholders can be used in a sys- 
tematic (and thus in a participatory, interaction-oriented and fair) decision-making process for health management that can be applied in other decision-making situations (e.g. hygiene or emergency management). As a theoretical contribution, the bridging of the implementation gap with the developed priority concept and its integration in an operational HRM model for German practitioners' surgeries with the psychological contract as core are discussed.

DOI: $10.46289 / \mathrm{BNGH} 1478$ 


\section{Introduction}

Health management is the central concept of this research. Health management itself is embedded in wider concepts like quality management, performance management and human resource management. These management concepts are adopted in companies and organizations of every size. This chapter introduces these concepts and their relevance for general practitioners' surgeries and how they aided the development of the research questions.

The idea for this research was developed during the practical work as a consultant, and the work with general practitioners' surgeries. The underlying idea of this approach was to use the knowledge of stakeholders within an organisation and their ideas for activities to make optimal decisions concerning employees' health management.

In Germany, a social market economy is practiced. The principle of the free market is associated with a social balance. The aim of this social and economic policy model is "... to combine free initiative with a social progress secured by economic performance based on the competitive economy." (Müller-Armack, 1976 - translated by the author). Social market economy and growing environmental issues are increasingly determining corporate social responsibility, leading to policy-specific regulations to ensure the health and well-being of all employees. This contextual background is shown in section 1. In doing so five areas related to health care management were outlined, which in varying degrees of intensity influence the daily decisions and priorities.

In section 2 these five areas are applied to the specific case of German general practitioners' surgeries. First, a description of the structure and size of German general practitioners' surgeries is given, and then the German national prevention strategy (§ 20d SGB V) and the prevention act (PrävG, 2015) as well as other health management related regulations are presented. An explanation of the significance for the employees is given.

Walter (2007) conducted a study on quality management and standardization of health management goals and procedures. In this study twelve goals for corporate health management in four principal areas are defined. This framework is used in the present research to anchor questions of health management. Walter (2007) evaluates management practices in small and medium-sized enterprises and recommends promoting the improvement of well-being and general health at work as a step towards an integrated and comprehensive health management system.

The subsection "Diseases and disease prevention at workplace" takes a closer look to this goal. There are five main groups of diseases which are arranged and classified according to the International Statistical Classification of Diseases and Related 
Health Problems (WHO, 2016) catalogue of the World Health Organisation (International Statistical Classification of Diseases and Related Health Problems, 2016). For these classifications validated statistical data is available (i.e. the number of absent days caused by the respective disease). Three of these five groups are finally used to derive concrete activities for the physician's assistants.

The last subsection applies the general decision-making model to the specifics of general practitioners' surgeries thus to assist health management decision-making and prioritising.

Section 3 of this chapter transforms the points above into research questions. And finally, the last section gives an overview of the following chapters.

\subsection{Contextual Background}

The following five areas form the context background and reflect the social, the operational, the economic, the medical and the management point of view. They are the basis for the systematic literature review in chapter 3 .

\subsubsection{Area 1: General health care management}

The idea of health care management means that the health of the population can be sustained, encouraged and managed through social and social policy measures. This also includes political measures which affect health management for the whole society. The political rules change regularly, influenced by different stakeholders. They are subject of a comprehensive social security code of the German government legislation (SGB I-XII, which is the social security and welfare legislation).

There are two issues that have emerged in recent years: the demographic change and the German national prevention strategy. The latter arises from the so-called Prevention Act (PrävG, 2015), which is a law on strengthening of health promotion and prevention. The intention is to develop measures to prevent diseases (prevention), and to promote health and early detection.

The demographic change in Germany consists of an older society and older employees in companies. The present retirement age is still rising (OECD; Pensions at a Glance 2011: Retirement-income Systems in OECD and G20 Countries Complete Edition, 2011). The rate of absent days of older employees (50 or more years) evaluated on the basis of the figures of the German statutory health insurances tends to be higher than the one of younger employees (Brussig and Ahlers, 2007). Therefore, companies have to care more about the health of their employees to keep the absence caused by sickness as low as possible.

The Prevention Act (PrävG, 2015) extends and modifies the SGB as it picks up on the living environments (schools, day-care centres, businesses, nursing homes) of the population and develops coordinated concepts tailored to these environments ("set- 
ting approach"). Physicians can make recommendations for prevention and inform about services from providers as adult education centres, sports clubs and fitness centres. The health insurance companies fund prevention courses, if the courses fulfil the quality standards of the statutory health insurance for $\$ 20$ SGB V.

\subsubsection{Area 2: Corporate health management}

While general health care management is the responsibility of the entire society, Corporate Health Management focuses on health and health promotion at work.

Occupational health protection deals with the effects of work on the health of workers. The aim is the prevention of work-related health problems and the prevention of occupational diseases. The statutory basis is the German Occupational Safety and Health Act (ArbSchG, latest amendment 2015) by which the employer is obliged to take the necessary measures of occupational health and safety, monitor their efficacy and, if necessary, adapt them ( $\$ 3$ ArbSchG). The work must be designed in such a way as to avoid a threat to life and to physical and psychological health and to minimize the remaining risks ( $\$ 4(1)$ ArbSchG). It is distinguished between prevention of the circumstances and behavioural prevention.

The prevention of circumstances deals with the working environment and working conditions. The employer has to assess the dangers emerging from handling hazardous substances, exposure to noise as well as the risks of physical and psychological stress and to eliminate or reduce these by appropriate measures.

Behavioural prevention aims at the behaviour of the individual employee. Information on health hazards and health-promoting behaviour is intended to prevent diseases.

The German Act on Occupational Health and Safety Professionals and other occupational safety and health professionals (ASiG, last amended in 2013) regulates the appointment of occupational physicians, safety engineers and other specialists in occupational safety. Big organisations and companies with more than 250 employees have their own occupational physicians who work fulltime for the company. An organisation with more than 51 employees has to engage an occupational physician. For smaller companies there are special regulations according to the regulations of the German Social Accident Insurance (DGUV). According to this, the entrepreneur with less than 51 employees after a motivation and information training can limit the safety-related care by an external freelance specialist for work safety or intercompany services to self-determined needs.

The management of health and safety measures - i.e. planning, organization, implementation and control - is not a voluntary entrepreneurial initiative but based on legal requirements. Only when it comes to the health-promoting design of jobs, workflow, structures and organization is it justified to speak of a company's health management. 


\subsubsection{Area 3: Business performance and quality in health management}

With the introduction of an occupational health management system, it soon became clear that good health, work satisfaction and a pleasant working climate could be success factors for more quality, higher performance and improved competitiveness. As a further goal of the company's health management, it was also desirable to prove the success of the business by means of suitable key indicators according to Badura, Greiner et al. (2008).

Several big sized companies as stated by Webendörfer (2011) started their own health management programs independent from governmental initiatives. The use of health management can lead to a competitive advantage. Following Maar, Fricker et al. (2011) professional health care management is likely to benefit for all organisations regardless of their size.

But other scientific findings on effects of workplace health promotion interventions are contradictory. Some researchers (Kallestål eds, 2004; Ozminkowski et al., 2002; Pelletier, 1993 - cited in Larsson, Landstad and Vinberg, 2009) cannot confirm a relationship between investments in workplace health promotion and performance improvements; others (Aldana, 2001; Jensen and Roos, 2005 - cited in Larsson et al., 2009) see strong correlations between health-related activities and productivity.

The number of absence days has proved to be the most important indicator for measuring the effect of health management measures according to Grobe, Dörning et al. (2011). In Germany validated data of absence days is provided by health insurance companies.

\subsubsection{Area 4: Diseases and disease prevention at the workplace}

This area shows the medical view of occupational health management. A distinction is made here between the disease-causing, symptomatic view (pathogenesis) and the health-promoting view (salutogenesis). According to Helmenstein, Kleissner et al. (2004) the term salutogenesis was introduced by Antonovsky (1997) as opposed to the classical concept of pathogenesis which deals with trying to treat diseases and the causes. Whereas salutogenesis is based on the principle of "staying healthy despite stress and strain" and the prevention of ill-health, pathogenesis is more concerned with "suffering from stress and strain" and the treatment of ill-health. While the classic treatment model only seeks the absence of disease, the wellness model sees the complete well-being as a goal of health promotion.

The salutogenetic conceptions have significantly influenced the conceptions of prevention. While the classical treatment model focuses on the risk factors that cause disease (left part of Figure 1), the wellness model also involves the recognition and strengthening of health-enhancing factors and resources (left and right part of Figure $1)$. 


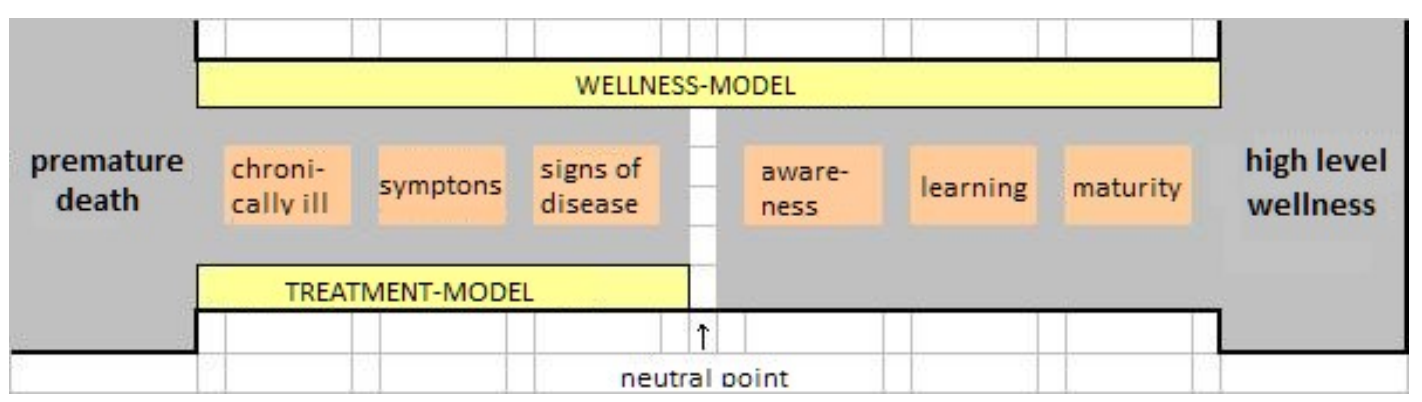

Figure 1: The salutogenetic conception - source: Hertel, 1992 cited in Helmenstein et al. (2004)

For this research, diseases which most frequently cause absent days according to health insurance statistics (Grobe, Dörning et al., 2011) are distinguished as follows:

- Musculoskeletal disorders

- Mental disorders

- Diseases of the respiratory system

- Injuries at the workplace

Fields of action and prevention relevant for the promotion of health and well-being at the workplace are distinguished according to the guidelines of the German government health insurance (Handschuch, Schreiner-Kürten et al., 2015):

- Nutritional behaviour

- Physical movement

- Coping with stress

- Drug prevention

It is characteristic for all four fields of action that they interlock health-promoting work organization and health-promoting work and lifestyle.

While treatment of diseases is largely regulated, preventive measures are based on voluntary action of both the employer and the employees. Before integrating preventive measures into long-term health management, it is recommended to examine each of them with regards to effectiveness and degree of participation and acceptance.

\subsubsection{Area 5: Decision-making}

Many measures such as nutritional counselling, physical movement or even vaccinations can be taken by companies to prevent illness and thus improve their health management (Sockoll, Kramer et al., 2006). These measures vary in effectiveness, expense and acceptation by the employees. 
As a rule, all proposed measures improve health management. However, resources are generally limited since not all measures can be implemented and conflicting objectives exist. For example: Should measures that are unpopular and inexpensive but effective - e.g. avoidance of alcohol abuse - rather be promoted than measures that are popular, but more expensive but may not be effective - e.g. yoga classes in the lunch break? But objectives cannot only lead to conflicts, they can strengthen each other and be complementary, also. Thus, an increase in physical mobility often leads to a reduction in stress levels.

Decision-making means to choose between evaluated alternatives. Alternatives, i.e. all measures for health promotion, are compared with each other and evaluated from the point of view of the different decision criteria acceptance, effectiveness and cost level. The ranking of the individual alternatives is used to recommend a priority implementation.

The process of decision-making is the core of this research. All collected theories and data are used to construct a concept, which can be used to optimize and prioritize the health management in line with the relevant objectives.

Figure 2 shows a multi-criteria decision-making process and visualizes how the whole research will lead to a concept for the development of a priority list. Following Roy (1981), cited by Ishizaka and Nemery (2013), development of a priority list is a typical ranking problem: "The options are ordered from best to worst by means of scores or pairwise comparisons, etc. ..." (Ishizaka and Nemery, 2013, p. 3).

First step in the process is to develop the decision criteria. For this, it is necessary that there is a great participation of the stakeholder; especially it is important that the employees who are important stakeholders are included. The stakeholders should also be involved in defining the weighting, which determines how relevant a decision criterion is for the achievement of the goal. To determine the extent to which an objective has been met, it is necessary to determine how this is to be measured or, if measurement is not possible, which indicators display the degree of goal achievement.

The right branch in Figure 2 is concerned with the development of alternatives and options. The first step is to generate a vast range of alternatives to allow the scope for action and decision-making being as complete as possible. In the next step, the alternatives are reduced to a manageable quantity by determining their feasibility. What remains are realistic alternatives. Finally, the extent to which a realistic alternative meets each decision criterion is evaluated by means of measurements or indicators.

The result of the assessment of each alternative for each decision criterion represents the attractiveness and performance in terms of the specific criterion - the ranking of alternatives regarding the specific decision criterion. If this scoring is combined with the weighting of the decision criteria, the overall scoring of the alternatives arises - or in the words of Ishizaka and Nemery (2013): a list of priorities. 
Elaboration of the decision criteria<smiles>CC1CC1</smiles>

Weighting of the decision criteria

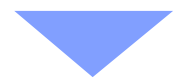

Measurability and indicators of the decision criteria
Creating a range of alternatives

Development of the realistic alternatives

Evaluating each alternative with the decision criteria

Generating a priority list

Sensibility analysis

and robustness analysis

Implementation

(resp. Customizing)

Figure 2: The multi-criteria decision-making model

Finally, the sensitivity analysis allows to observe how changes in weighting the decision criteria affect the order of priority. Using the robustness analysis, one can determine how long the order of priority determined remains valid, considering uncertainties in the weighting as shown by Ishizaka and Nemery (2013) and Zangemeister (2014). Implementation resp. customization of the decision-making model means that the practical approach is made transparent for the user and enables him to make changes on one's own, e.g. for additional decision-making criteria or new alternatives.

The next section transfers the previously described model to health management decisions in general practitioners' surgeries.

\subsection{German general practitioners' surgeries and health management}

In the following, German general practitioners' surgeries and their specifics are presented against the contextual background presented above with a focus on decisionmaking for health management. 
Responsible for the implementation of a health management system in German general practitioners' surgeries is normally the owner him/herself - the general practitioner. He/she is the general manager and the medical expert in one person. The physicians are not trained and experienced in management decision-making. Like comparable micro-enterprises they need highly standardized procedures and recommended activities. These standards should cover the entire process from identification of activities, the implementation of health improvement programs and the practical part for the day-to-day work, therefore the decision-making process itself is a key part of the research questions.

\subsubsection{The structure and size of German general practitioners' surgeries}

General practitioners' surgeries are an integral part of the German health care system. The individual physician's practice is usually an economic enterprise, which is led by a self-employed physician, as a rule the legal owner and sole holder. However, he/she has to practice under the legal framework and is accountable for his/her actions under the German law.

Such a freelance activity - as other scientific, artistic, teaching or educating professions - is not a trade under German law (GewStG §2). Income earned through medical activities is subject to income tax (EstG §18). In contrast to other freelancers, however, medical services provided by the physician are exempted from value added tax (UstG $\S 1)$. The uniform regulations of approval in all federal states regulate the objectives, content and the duration of the training as well as examinations and approval as a physician ( $̈$ ApprO $\S 1$ ). Physicians cannot be held liable for the success of their therapies and actions, but they can be sued for damages in the event of culpable, unauthorized action (BGB $\S 823$ ). In Germany, the practice of medical treatments is reserved only to approved physicians. Some special activities (such as blood sampling for laboratory tests) can also be carried out by medical assistants (MFA) (see below).

Each physician is a mandatory member of a medical chamber. As a public-law corporation, medical chambers are the bearers of the professional self-administration of the medical profession. The Medical Chambers are organized according to federal states. The Federal Chamber of Physicians is the working group of the provincial chamber. The physicians are a member of the state chamber in which they carry out their medical activity. Among the tasks of the seventeen medical chambers are the promotion of quality assurance measures and the organization of the training of medical assistants (MFAs).

In 2015, in Germany there were around 370,000 working physicians in total, $46 \%$ of which female. 150,000 are working with outpatients; about 120,000 of them were licensed for a physician's surgery. In total, there are some 54,000 general practitioners' surgeries. In addition, there are about 7,000 paediatric doctors' surgeries; the remaining approximately 59,000 surgeries are specialist surgeries. Following the phy- 
sician monitor on average, the general practitioner is fifty-five years old (infas, 2016, p. 55). The proportion of women is $44 \%$ (infas, 2016 , p. 56). The average general practitioner's surgery has 3.5 fulltime-jobs (infas, 2016). In the physician monitor there is no statement about the age structure and gender of the employees.

In the physician's office, patients are received, advised and treated. The patients are divided into so-called public health patients and private patients.

\subsubsection{Public health patients}

The public health patients belong to the statutory health insurance (GKV) or other insurance (pension, unemployment, accident, and nursing insurance) of the German social security system. In contrast to the institutions of statutory health insurance, private health insurance funds (PKV) are not public-law corporations, but private insurance companies which reimburse their clients, the private patient, according to the underlying insurance contract and tariff.

The statutory health insurance is a compulsory insurance for all employees who are not classified as insurance-free or who are not otherwise entitled to insurance in case of illness. For the treatment of public health patients, the doctor requires an authorization, which means that he belongs to one of the 17 Associations of Statutory Health Insurance Physicians (KVs), which, according to the social act (SGB), perform the contractual care of all insured persons of the GKV (§ 77). The residence permissions for physicians who wish to participate in the health care system are established by means of an authorization committee. They are limited and are divided into quotas oriented to requirements. In Germany, statutory patients can choose between more than 200 health insurance companies. The health insurance companies conclude contracts with the KVs, which are binding for the individual doctor. Physicians do not charge the individual patient or a single health insurance, but instead they are obliged to a collective agreement for the respective $\mathrm{KV}$ area in which the total remuneration has been agreed on. The distribution, billing and questions of profitability and practice are the subject of medical self-administration within the framework of the funded associations (KVs). The benefits to be provided within the framework of the statutory health insurance are precisely defined in the social act (SGB) and remunerated by the health insurance funds as described above. In addition to these compulsory benefits, which equal for all health insurance companies, health insurance funds can offer supplementary benefits within the framework of elective rates.

Almost every physician is licensed for social health insurance (SHI). The proportion of private patients in general practice is around $8 \%$. This is supported in a study by infas (2016): From October to December 2015, general practitioners' surgeries had an average of 150 private patients compared to 1,615 SHI-patients (p. 49, 50). In contrast to this low percentage of $8.5 \%$ the share of revenues of private patients (including private supplementary offers) is around 13\% following (infas, 2016). 


\subsubsection{Private patients}

In the case of private patients, the physicians directly charge the patients. The patient then usually passes the invoice onto his private health insurance (PKV), which assumes the expenses within the agreed scope of the insurance contract (tariff). This is regulated in the Insurance Contract Law (VVG §192). In Germany, physicians are not entitled to free pricing. They are tied to the physicians' fees schedule (GOÄ). Since the GOÄ offers a certain billing margin (up to 3.5 times the rate of charge) the treatment of PKV patients is more lucrative than that of GKV patients. Private services also include the so-called Individual Health Services (IGeL) - these are medical services which must not be covered by the health insurance funds because they go beyond what is considered to be adequate, appropriate and economic care according to $\S 92 \mathrm{SGB} \mathrm{V}$.

\subsubsection{Medical assistants (MFAs)}

In addition to the doctor, the so-called medical assistants (MFA - until 2006: doctor's receptionist) work in the physician's office. They are in the focus of this research.

MFAs carry out tasks of medical assistance (e.g. blood sampling, injections, etc.) and take care of the practice organization (appointment agreement, billing, correspondence, recipe printing, patient call, etc.). Since 2006, MFAs undergo a three-year apprenticeship in the dual system (doctor's office and vocational college). Contents and implementation are regulated by a training regulation and an educational framework (KMK, 2006) and a framework curriculum (KMK, 2005). The "Certified Specialist for Outpatient Medical Care" as well as the "Certified Specialist in Health Care and Social Work" are available as further regulated training courses.

Although MFAs are among the largest occupational groups in outpatient care, there are hardly any studies on workload and health hazards. In a qualitative study, VuEickmann and Loerbroks (2016) conducted 26 depth interviews and published the results under the title "In the doctor's office nothing works without us": "A high workload (e.g., patient emergencies, personal shortcomings), frequent interruptions (e.g., by telephone, urgent concerns) and multitasking (especially at the reception of a physician's surgery) were found as occupational stressors. The social aspects of the profession (such as dealing with people, positive feedback from the patients), the different activities (e.g., office activity, patient contact) as well as the medical activities were identified as occupational resources. In addition, MFAs are strongly dissatisfied with their low salary and often lack recognition by employers and society." (translated by the author).

The latest current health report (BMG: Health system facts, 2016, p. 97) shows that 678,000 persons work as doctors' receptionists and assistants, of which 417,000 are medical assistants (MFAs). Of these, an estimated 194,000 work in general practitioners' surgeries, with $98.3 \%$ of MFAs are being females. The average age is 39.3 years. 


\subsubsection{Competition of medical service offers}

At first glance, the market for medical services appears to be regulated. From the perspective of the general practitioners' practice, however, the following competitors exist:

- Group practices and practice sharing (18\%): generally, offer a wider range of products and can organize resources more economically.

- Specialists' surgeries (21\%): concentrate on their specialist area, can standardize their work processes better and spend less time on economical not so interesting house visits. In addition, the number of patients treated on average per day is significantly lower.

- Medical care centres (15\%): bundling of medical specialists for different fields allows a wide range of services and high competence. Lean organization may bring economic advantage.

In brackets, the percentage share of private revenue following infas (2016) is shown as an indicator of competitiveness (GPs - 13\% - 2.2.2.1). The decision for a certain practice, especially in the case of non-compulsory preventive services, is strongly influenced by friendliness of the MFAs.

\subsubsection{Area 1: General health care management}

The focus of this research is on the free-practicing general practitioners with their own practice. The name "general practitioner" indicates the holistic approach that he/she provides the health care regardless of the age, sex, illness, organ of the patient, and, if necessary, passes it on to a specialist. As a rule, the general practitioners in the German health care system are, in addition to the pharmacies, the first point of contact in medical matters. From the point of view of health management, this research is not concerned with the purely medical services, but only the so-called preventative services, which are appropriate to prevent diseases, and which have a beneficial effect on health.

As described in Regus (2012), the prevention services are divided into two categories. The first category is the so-called health screening (secondary prevention) for the early detection of diseases such as, for example, blood glucose, cholesterol, blood pressure, etc. They are not the subject of this research since they cannot be attributed to health management as purely medical activities. This study focuses on the second category, the so-called behavioural health risks (primary prevention), such as dietary advice or smoking cessation. A third category is tertiary prevention, which is also not the focus of this study. Tertiary prevention is aimed at preventing the consequences of a disease or its progress (Schwartz and Walter, 2003, p. 189).

„In many respects, the physicians are in a favourable position for a commitment to behavioural prevention (primary prevention): their comprehensive knowledge of the 
patient and his/her social living conditions, as well as the possibility of long-term care, increase the success prospects of consultation. "(Regus, 2012, p. 29ff - translated by the author). On the other hand, the doctor often lacks the time for the consultation. Only $9 \%$ of the physicians surveyed gave a clear "yes" to the question about sufficient temporal resources according to (Regus, 2012). Most physicians are willing to delegate measures of primary prevention to MFAs. This is of importance for this research:

- If primary prevention is carried out by the MFAs themselves, the likelihood that health management will improve in one's own general practitioner's office is increasing since it is assumed that counselling other persons also has a repercussion on their own health behaviour.

- As described in subsection 2.1.1 (General health care management), primary prevention measures can also be provided by other organizations than physicians' offices within the framework of the prevention law. If the MFAs gain competence in this area, the range of services offered by the individual general practitioner's surgery can be expanded and a competitive advantage (professional competence) can be achieved.

- Within the context of the setting approach of the national prevention strategy (2.1.1) primary prevention services are also to be provided in places other than physician's practice. If the MFA accepts the delegation of primary prevention as her task, the services of general practitioners' practices can also be provided by the MFAs in other places (e.g. nursing homes, schools, etc.). This would also be compatible with the training and training measures mentioned above.

- As stated at the beginning of this section, GPs are obliged to attend house visits. A part of these home visits, especially when primary prevention is combined with measures of secondary and tertiary prevention, can then also be exercised by the MFAs to relieve the general practitioner.

These points are directly related to the research question because they influence the preference for certain preventive measures:

- Which of these measures are already practised and which are preferred by the staff of the general practitioners' surgeries?

\subsubsection{Area 2: Corporate health management}

Following the recommendation of the European Union Commission (2003) the typical German practitioner's surgery is classified as micro-enterprise: Less than $10 \mathrm{em}-$ ployees and maximum revenue or total assets of two million Euro. On average, the individual general practitioner's surgery has 3.5 full-time employees (infas, 2016, p. 
47). The small size frees the employer, namely the doctor, but not to design the work in such a way as to avoid a threat to life as well as to physical and psychological health and to minimize the remaining risks (2.1.2). But in contrast to large companies, general practitioners' surgeries are not obliged to engage an occupation physician or to use professional occupational health care services.

If a doctor decides to introduce a health management system, often from the idea of an exemplary function, they are by no means as much resources available as in larger companies. To act economically, the use of standards and patterns is recommended by Walter (2007). She proposes the following objectives as a standard for small and medium-sized enterprises:

- Development and permanent anchoring of the management system

- Strengthening the social capital and human capital

- Improvement of well-being and health

- Improvement of productivity, quality and profitability

Walter's proposal was designed for small and medium-sized enterprises with the idea of high-level standardization. Especially for small and medium-sized enterprises, the standardization idea is of immense importance, since "the standard setting helps to improve the intra- and interprofessional cooperation in occupational health management and thus the effectiveness and efficiency in occupational health management has increased " (Walter, 2007, p.130 - translated by the author). The concept's viability has been evaluated in several small and medium-sized enterprises, and since there is no comparable standard for micro-enterprises, Walter's approach to general practitioners' surgeries has been adopted. This was even more obvious to general practitioners' surgeries since the standard was designed as a guideline. "The term 'guideline' deliberately expresses the orientation towards the concept of medical guidelines. " (Walter, 2007, p.129 - translated by the author).

Regarding the size, a concession was made: A general practitioner's surgery does not have a sprawling management system, so that the establishment of operational policy conditions and the structure of the framework conditions are essentially the person of the physician. However, they should not see health management as a one-time activity, but rather an usual permanent management procedure (Schirrmann, 2017).

The social capital and the human capital of a medical practice are essential factors that influence work satisfaction and motivation. Starting with job design, the design of the workflow, teamwork and the development of a vision, and personal health potentials are striven for. The improvement of well-being and health includes both increasing psychosocial well-being, improving physical health, and reducing risk factors. At the last point, a purely medical risk factor, such as risk of infection or radiation exposure, and the general risk factors such as high blood pressure and obesity are distinguished for a general practitioner's surgery. The purely medical risk 
factors are not the subject of this research, since they are regulated in labour protection legislation. The improvement of productivity, quality and profitability will be dealt with in the next subsection.

Usually, the first step in the introduction of a health management system would be to build up and establish permanently a management system, and then the creation of social and human capital through job and workflow design. For general practitioners' surgeries, however, this study suggests that the first step is to promote personal health potentials. This is explained in more detail in the section "Deriving priorities for general practitioners' surgeries" (cf. 3.3.3) and by the in-depth interviews with physicians.

Transferring the concept for the goals and results of a health management system designed for small and medium-size enterprises to micro-enterprises the following research questions arise:

- What interactions should the general practitioner (as owner) consider if he/she wishes to set priorities for the introduction of a health management system?

\subsubsection{Area 3: Business performance and quality management}

The Balanced Scorecard (BSC) has proven itself as a tool for measuring the performance of organizations following Kaplan and Norton (1996). To assess performance, the organizations are examined in four distinct aspects:

- Human resource perspective

- Quality and process perspective

- Customer perspective

- Financial perspective

The human resource perspective is the main starting point for this research. It covers the qualifications and the competencies of the MFAs as well as their motivation, commitment and work satisfaction. The parameters of the quality and process perspective are largely defined in the Quality Management Guidelines (QM-RL) for SHI-accredited physician published by G-BA. The Federal Joint Committee (G-BA) is the highest decision-making body of the joint self-government of physicians, dentists, hospitals and health insurance funds in Germany. The primary goal is to achieve a permanent increase in quality in patient care. In addition, quality management should also help to increase the satisfaction of all parties involved in the process; In general practitioners' practice, those parties involved in the process are the MFAs. Within the next three years (until 2019), the SHI-accredited physician is obliged to introduce and refine methods and tools for increasing the structure, process and result quality (Bundesanzeiger, 2016, p. 12). The customer perspective in a 
general practitioner' surgery is the patient's perspective. While the quality management guidelines mainly focus on medical quality (e.g. therapy success, error prevention, hygiene measures, etc.), patient satisfaction is more likely to be attributed to the practice organization, waiting times and workflows. A prominent level of patient satisfaction suggests more private patients (cf. 2.2.1), whose share improves the $f i$ nancial perspective. There are fixed regulations for the financial framework of a general practitioner's practice. These apply to both the income and the cost side. As a result, profitability can only be substantially increased by the productivity or proportion of private patients.

The general environment in which the author operates, confirmed by numerous discussions with general practitioners outside this research, reveal the overall relationships are as follows: Primary prevention measures are part of human resource management. First, a reduction in missing days is expected. In this way, they directly affect productivity thus the financial performance. If the number of absent days increases, more efforts must be made for the same service (e.g. remuneration of substitutes). Conversely, fewer absent days lead to a higher productivity (higher patient throughput). An increase in the absenteeism also has a negative effect on quality and processes. If the absent days are compensated, for example, by increased use of colleagues, the error probability increases because the workload of other team members increases and thus the risk of inaccurate treatment increases as stated by Gehring and Schwappach (2014). There is also less time for patient information and enlightenment, a quality aspect that is explicitly required by Quality Management Guidelines (QM-RL) for SHI-accredited physician following G-BA (Bundesanzeiger, 2016). Absent days directly affect the customer satisfaction, which means patient satisfaction: Waiting times are prolonged, the agreement of treatment appointments is impaired, and the patients do not find their usual environment. This is a situation that is particularly difficult in the case of sick people.

The performance-relevant reduction of absent days is directly related to the research question:

- What research studies indicate a strong link between measures of health promotion and a direct result in reduction of absenteeism?

But not only the missing days, but also other indirect, performance-relevant effects of the promotion of personal health potentials should be considered. As a rule, primary prevention measures also lead to more satisfied and motivated employees, a better working environment and a lower level of stress (Bruch and Kowalevski, 2013). However, the prerequisite here is that the employees, namely the MFAs, are involved in the planning of the measures and accept them. Again, these interrelationships are taken up in the afore mentioned research question: 
- Which of these measures are already practised and which are preferred by the staff of the general practitioners' surgeries?

A detailed discussion of the performance relationships beyond the primary prevention measures can be found in chapter 7 "Discussions".

\subsubsection{Area 4: Diseases and disease prevention at the workplace}

Already in subsection 2.1.4 "Diseases and disease prevention at the workplace" the diseases, which mainly lead to absent days, were presented. In addition, it has been shown which health-promoting measures may help to prevent these diseases. This section deals with the specific features in the medical practice, as described, for example, in the 'Round table' publication (Runder Tisch Hannover a, 2014) or Steinert and Wittmann (2010). Compared to other services in the non-medical sector, the activity in medical practices is as follows:

- Higher risk of infection (aero gene infections, contact and lubrication infections)

- More skin diseases (frequent hand washing and wearing of protective gloves strain the skin, so-called dampening work)

- Higher risk of injury, e.g. by so-called needle-stick injuries (Hepatitis B and C, HIV)

- Higher risk of contact with hazardous substances (medicines, disinfectants, bio-waste, ...)

Except for the first point (infection risk), all measures are not part of the health management in a strict sense. They must be treated according to the statutory rules for occupational health and safety. Therefore, the prevention measures referred to in subsection 2.1.4 need not be specifically extended from the point of view of a general practitioner's surgery. However, disease prevention by vaccination is of importance in medical practice. The risk of infection is particularly high through contact with the patients. Infectious diseases are a good example of the fact that one and the same disease can contribute to both absenteeism and presentism. Whereas absenteeism means to be absent from the workplace caused by illness, presentism means to be present at the workplace despite illness (Steinke and Badura, 2011). The consequences of presentism are often worse than those of absenteeism. If e.g. employees with flu go to work, the risk of infecting other employees and patients increases, they are limited in performance and productivity and finally they endanger their own health in the long term.

Compared to other non-medical service companies, general practitioners' surgeries have the advantage that, as a rule, the know-how for primary prevention is in the home. Figure 3 shows the results of a study on the role of GPs in primary prevention 
(Regus, 2012). According to this, the physicians $(\mathrm{N}=274)$ particularly value their own consultancy competence for two of the prevention areas selected in subsection 2.1.4: physical exercise and nutritional behaviour. Over $80 \%$ value their consultancy skills on "physical exercise" with "very good" or "good". Almost $80 \%$ have the same self-assessment on "nutritional behaviour".

This self-assessment of the general practitioners should not be overlooked when drawing up a prioritized approach to health management.

Within the prevention measures nutrition and physical activity there are different forms and variations. So, physical activities may be carried out directly at the workplace or in the gym. In chapter 2 "Literature Review" the different forms and corresponding studies are recorded. The diverse sizes of organizations and companies must be considered, too.

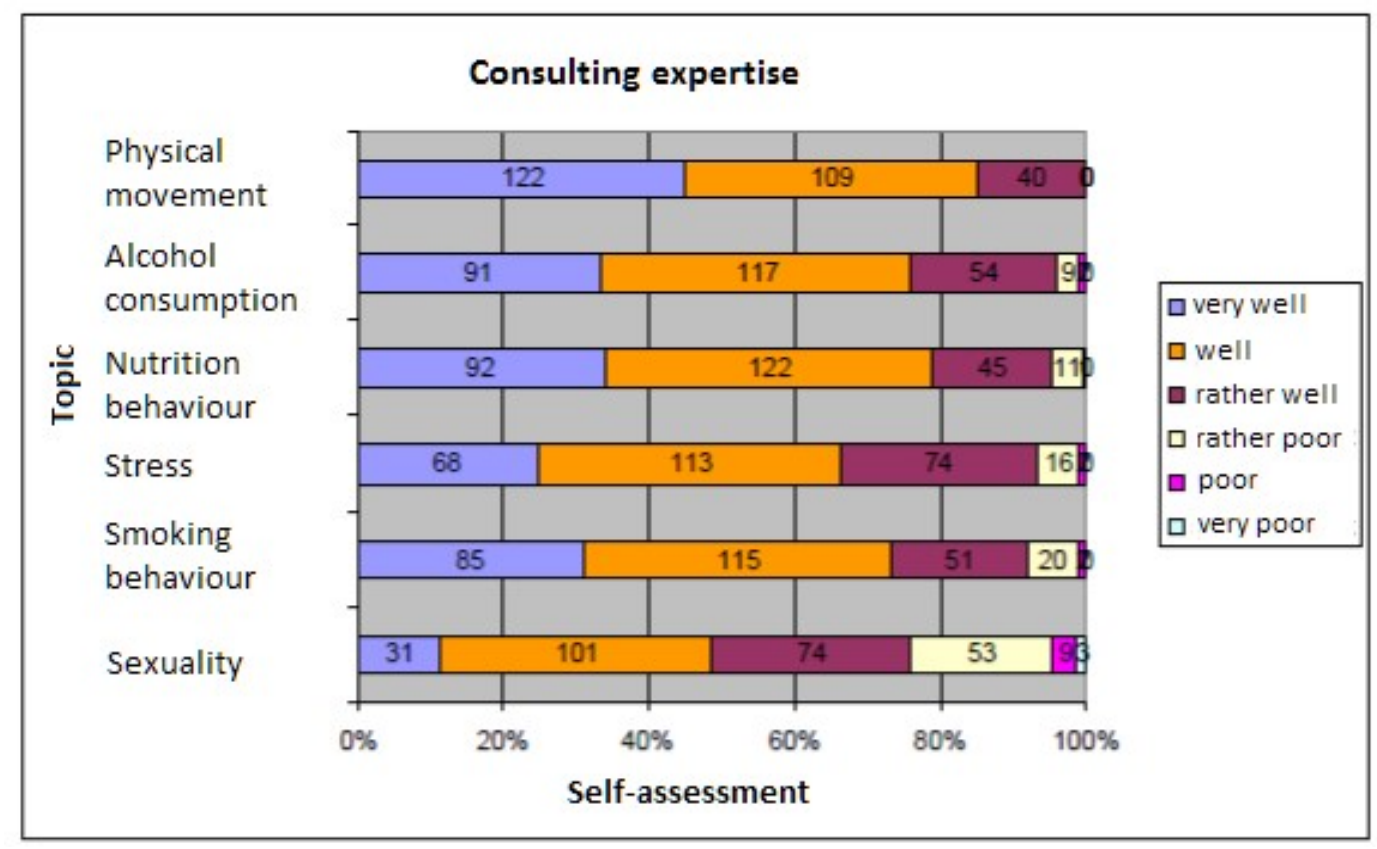

Figure 3: Self-assessment of the consultancy competence of the general practitioners in the most important primary prevention areas - source: (Regus, 2012)

\subsubsection{Area 5: Decision-making}

Decision-making itself is a part of the day-to-day activities for physicians' practices. One of the typical decision-making situations is the decision to become selfemployed and decide to establish a practice. While the founding the medical and in the further course of operating the office, location, investment, financing and personnel decisions are to be taken. And in the exercise of the medical activity itself, decisions for diagnosis and therapy belong to the workplace. 
Much less experience has general practitioners' surgeries in long-term strategic decisions such as marketing activities, leadership concepts and, indeed, health management as part of human resource management. For a decision-making procedure to be used in a general practitioner's surgery, it should meet the following requirements:

- Low expenditure on time and easy to handle. General practitioners, working in solo practice, have already the highest workload with an average of 53.6 hours per week compared to other practice forms. For the guidance of the practice team remain only 1.6 hours per week, for other activities 2 hours per week following (infas, 2016).

- Well-founded, transparent, evident and suitable be integrated into the guidelines for prevention (GKV-Spitzenverband, 2014).

- Little bureaucracy: presently physicians and MFAs have to deal with 21 legal regulations and statuary requirements for employee and patient protection (Runder Tisch Hannover b, 2014).

- Standardized but adaptable both to the physician's own decision criteria and alternatives for measures.

The proposed procedure as outlined in the decision- making model shown in Figure 2 could fulfil the requirement by elaboration of the priorities list for health promotion activities and human resource management. The elaboration of the priorities list follows the multi-criteria decision-making model shown in Figure 2. The author proposes, that the most important decision criterion is the acceptance of the measures by the MFAs, followed by the cost forecast and the assessment of effectiveness. The great number of possible primary prevention measures are analysed individually and, by using exclusion criteria, compressed to the realistic and manageable number of ten alternatives.

In the last step, however, the implementation, there is a specific feature that should be considered. As described in subsection 2.2.5, general practitioners are obliged to carry out quality management. In this step of the decision-making model, it is therefore necessary to examine whether the health management of the general practitioner's surgery should be embedded into this concept or whether it should be designed as a "stand-alone concept" with an interface to quality management.

A central issue for the general practitioners is the question of how, with their tight time budget and the high workload, they can develop a viable concept for primary prevention within the framework of mandatory quality management, which can also be implemented quickly and meets expectations to a company health management. This includes the reduction of absences and possible performance improvements through the concept. This expectation is the focus of the concept of priorities to be developed. 


\subsection{Research questions and the way to answer them}

The following research questions derived from the issues identified and are discussed in the sections above:

1. What research studies indicate a strong link between measures of health promotion and a direct result in reduction of absenteeism?

2. Which of these measures are already practised and which are preferred by the staff of the general practitioners' surgeries?

3. On what basis can the implementation of these activities be prioritized by a multiple criteria decision-making model, which considers relevant stakeholder perspectives?

4. How can the procedure of decision-making be adapted to the specific conditions of an individual general practitioners' surgery?

5. What approaches should the general practitioner (as owner) consider if he/she wishes to set priorities for the introduction of a health management system?

The first question is answered mainly by a systematic literature review, which not only reveals the relevant primary prevention studies, but also examines them in an overall concept that provides a link between HR practices such as health management and organizational performance. The identified areas of promoting personal health potentials (primary prevention) are broken down to concrete and practicable proposals, which are used in a questionnaire for an employee attitude survey to answer the second question. The third question regarding priorities is answered by means of standardized multi-criteria decision software. The results from the literature analysis, the MFA survey as well as the interviews with physicians are considered. The sensitivity and robustness analysis provide a first approach to develop adaptive possibilities of the model assumptions for practice-specific conditions and thus to answer the fourth question. In answering the last question, the interviews with the physicians play a crucial role by discussing the extent to which the proposed health management measures can be integrated into a quality management concept.

\subsection{Overview: The chapters of this research}

The chapter Literature review contains three main topics: absenteeism, performance, and implementation. Models and activities to reduce absenteeism are discussed. They show determinants of absenteeism and open up a deeper understanding. A comprehensive literature review identifies the health management measures, in particular those of primary prevention, for which a reduction in absence is provable. Next section is about embedding the targeted prevention measures as a HR practice in a theoretical framework that discusses the relationship between HR practices - psychological contract - organizational performance, especially in general practitioners' surgeries. The priority concept as a result of a decision-making process and its references to 
the psychological contract are discussed as an essential prerequisite for implementation, so that the expected reductions in absences and hoped-for performance increase may take place.

The chapter on Methodology focuses on the multi-criteria decision-making model presented in Figure 2. While in the literature search the greatest possible variety of alternatives was chosen, the alternatives for a general practitioner's surgery are focussed to ten measures, the success of which can be substantiated. These measures are used as core items of the MFA survey.

Firstly, a questionnaire for the MFAs was developed using the multi-criteria decision-making model for acceptance of measures of promotion personal health potentials (primary prevention) i.e.: the cost structure and the expected effectiveness of the measures are elaborated. This forms the prerequisites for creating a list of priorities. For this purpose, a number of software products are available. M-MACBETH (Measuring Attractiveness by a Categorical Based Evaluation Technique) was decided upon most suitable. Finally, a semi-structured questionnaire was developed for an in-depth, face-to-face interviews with general practitioners.

In the chapter Research Findings of the MFA Survey, the results of the evaluation of questionnaires are presented, analysed and interpreted. The questionnaire consists of three parts. Part 1 is about demographics, Parts 2 is about the general practitioner's surgery, Part 3 is about delving into the issue of health management. In addition to the descriptive evaluation of measure preferences, hypotheses about relationships are tested for significance, such as: "Have MFAs of general practitioners' surgeries with existing health activities other preferences than those without?", "Do the preferences of MFAs with up-to-date vaccination status differ from those MFAs with an incomplete vaccination status?". The different preventive measures allow groupings and give arise to questions such as "What is the share of MFAs with low/high movement preference with low/high nutritional preference?" or "Is there a correlation between the preference for movement and that for healthy nutrition?".

The findings of the MFA survey would form a basis for the introduction of health management activities, as the acceptance and the resulting motivation are decisive for the success. In the chapter Research findings for the development of a priority concept, costs and effectiveness are added to this employee perspective as further stakeholder interests. An assessment of the expected costs and an assessment of the effectiveness are therefore made for each of the ten recommended measures. With these inputs, a priority list is created in accordance with the multi-criteria decisionmaking model presented in Figure 2. A sensitivity analysis and a robustness check are carried out with the aid of the M-MACBETH software before an implementation. This is done to check whether the order of priorities varies with a change in weighting (sensitivity) or with possible differences in assessment (robustness).

The Discussion starts with the last step of the multi-criteria decision-making model, the implementation. The concept proposed as a standard is discussed regarding its 
adaptability to the individual general practitioner's surgery. Physicians were interviewed regarding three basically different approaches for the implementation of this concept onto the management system of general practitioners' surgeries. Recommended measures can be realised in form of single initiatives, as a first step to an introduction of health management or as part of the existing quality management. Based on the "target system of health management in general practitioners' surgeries", the contribution of the goals of the proposed measures will be discussed. Likewise, the results and their anchoring in the theoretical framework HR Practices - psychological contract - organizational performance are discussed. The framework itself will be extended by current developments in the field of MFAs and the reorientation of general practitioners' surgeries.

The chapter on Conclusions starts with a presentation of the contribution to knowledge in the categories empirical research, methodology and practice about German general practitioners' surgeries. It also mentions the limitations of this study. Furthermore, for the developed concepts it is shown to what extent they can be transferred to other work areas of MFAs and which transfer possibilities are considered for use in SMEs.

\subsection{Summary}

The aim of this first chapter was to break down a general view of health management on the environment of a general practitioner's surgery, focusing on the specific concerns of primary prevention of MFAs. It was pointed out that the measures for primary prevention in the vocational and professional field of MFAs play a special role in comparison to almost all other fields of work and activity. They can be part of the competence of the MFAs and be perceived by them.

Thus, promoting health as a component of health management can make a significant contribution to the performance of a general practitioner's surgery. This was demonstrated and clarified by the four perspectives of the Balanced Scorecard (Kaplan and Norton, 1996).

It was also shown which measures are fundamentally suitable for disease prevention and which specific features such as an increased risk of infection and contamination exist for MFAs. Due to the small size of general practitioners' surgeries, illnesses can lead to significant disruptions of the practice and even endanger the safety of patients.

To be able to select and prioritize possible measures systematically and transparently, a multicriteria decision making model was introduced. In the following, it serves as a common thread that gradually leads to the development of a priority concept. Five central research questions are related to this model.

Which measures might be expected to reduce sick leave days - one of the possible main goals of health management - is the topic of the following chapter. It not only 
offers a systematic literature search, but also arranges the results in relevant target systems and process models. 


\section{Literature review}

In this thesis, the author sets out to develop a priority concept of activities promoting personal health potentials for German general practitioners' surgeries. It is a health management measure that is implemented in a micro-enterprise and should lead to a reduction of absenteeism and an increase in performance. From these points of view, the existing literature is reviewed.

Section 1 "Models and activities to reduce absenteeism" explains the general theoretical framework and background. The central importance of motivation was early analysed by Rhodes and Steers (1978) in their "model of employee attendance." It examines numerous other influencing factors, including health, its impact on attendance, and thus the performance. The author has therefore decided to use this model for a fundamental discussion of the conditions in German general practitioners' surgeries. The modified model of Rhodes and Steers, the "causal model of absenteeism" (Brooke, 1986), is particularly important for MFAs, as it sees primary prevention not just as a way to improve health status and thus reduce absenteeism, but also takes into account changes in role understanding and work organization circumstances. As part of health management, the last sub-section focuses on primary prevention measures that lead to a demonstrable reduction in absenteeism and which can be used by general practitioners' surgeries that do not have any HR departments and capacities.

The targeted measures to promote personal health potentials are common HR practices. Section 2 "A framework to increase performance in micro-enterprises" examines the impact of HR practices on organizational performance. The focus is on SMEs and within this group on micro-enterprises. Looking for HRM theories for SMEs, one quickly realizes that this area is under-researched and, because of its many peculiarities, creates difficulties for common HR theories. Atkinson (2005, 2008) therefore recommends personnel contract theory by Rousseau (1989) as a suitable framework that can be used for analysis regardless of size. The examples given in the literature focus on small and medium-sized enterprises. This section looks at how in a micro-enterprise, such as the physician's surgery, introducing primary prevention measures can influence components of the psychological contract. To discuss the overarching employment relationship, a more comprehensive framework linking HR practices, psychological contract, and performance is used (Katou, 2013). This framework, which uses a confirmatory factor analysis, identifies the variable "maintain high level of attendance" - known from the Rhodes and Steers model (1978) - as the main driver of employee's transactional obligations, so that general practitioners' surgeries has a promising approach here.

The transfer of HR practices from theory into practice is recognized as the "implementation gap" by many HRM researchers. This area, subject of Section 3, is also under-researched. Considerations for the fulfilment of the psychological contract as 
well as the development of the priority concept and its implementation are contributions to closing this gap. The basis for this is participative decision-making concept and a standardization proposal for health management in SMEs developed by Walter (2007).

\subsection{Models and activities to reduce absenteeism}

The Rhodes and Steers model (1978), which will be presented at the beginning of this section with reference to MFAs, combines, as stated by Brooks (1986, p. 346), two forms of absenteeism in one model, voluntary and involuntary absenteeism. It is assumed that the determinants of attendance motivation correspond to voluntary absenteeism, while the ability to attend is more associated with involuntary absenteeism.

The focus on Brooke's "causal model of absenteeism" (1986) as a modified model of Rhodes and Steers, which is discussed in sub-section 2 also with reference to MFAs, is on voluntary absenteeism, which is discussed in its dependence on the factors of satisfaction, job involvement, commitment and health status as well as alcohol involvement. In the relationship between health status and (voluntary) absenteeism, the mental aspect of health and well-being is therefore more important.

Involuntary absenteeism, which is caused primarily by physical disease, is treated in the third sub-section. Based on the symptoms of these diseases, which contribute the most to absences in Germany, measures are researched which demonstrate a reduction in absence in the context of health management and primary prevention. These measures form the basis for the data collection for the MFA survey.

\subsubsection{A model of employee attendance}

The management of absences and attendance, a core theme of this work, is an important part of the performance management, which in turn is a sub-area of human resource management. As a theoretical background, Rhodes and Steers' model of attendance is used (Rhodes and Steers, 1978). Model terms are marked in italics below.

The process model of the Employee Attendance offers numerous general advantages despite its age. There are a few reasons that justify this:

- From the point of view of the authors of the standard work on "Human Resource Management" is the process model of Rhodes and Steers: „... in our view this is the most useful of the process models" Torrington, Hall et al. (2017, p. 292).

- It is a process model, or in other words a dynamic model, since feedback is present. The authors themselves describe this property as a "cyclic nature of model" Rhodes and Steers (1978, p. 30). 
- The elaboration of the correlations was based on 104 empirical studies, so that a valid starting point existed. In the course of time, many studies have been conducted, which investigate individual relationships for individual sectors.

- With reference to this research should be stated: Measures of health management are ultimately measures for the improvement of Employee Attendance - (Hedges, 1973) and (Hill and Trist, 1955) cited in Rhodes \& Steers (1978, p. 22). In order to carry them through, they must be accepted by employees as stakeholders. In order to increase the likelihood of successful acceptance, knowledge of Personal Characteristics, including personal preferences, is required. The latter are the subject of this work within the framework of the MFA surveys and form the basis for important research hypotheses on the preference and decision model, e.g. "The advocacy (or rejection) of health management programs is age-independent." or "Do MFAs particularly prefer cost-intensive (or effective) measures?"

- With the factors Attendance motivation and Satisfaction in the model, two employee attitudes are introduced, which play a central role in fulfilling the psychological contract, which is discussed in the next section.

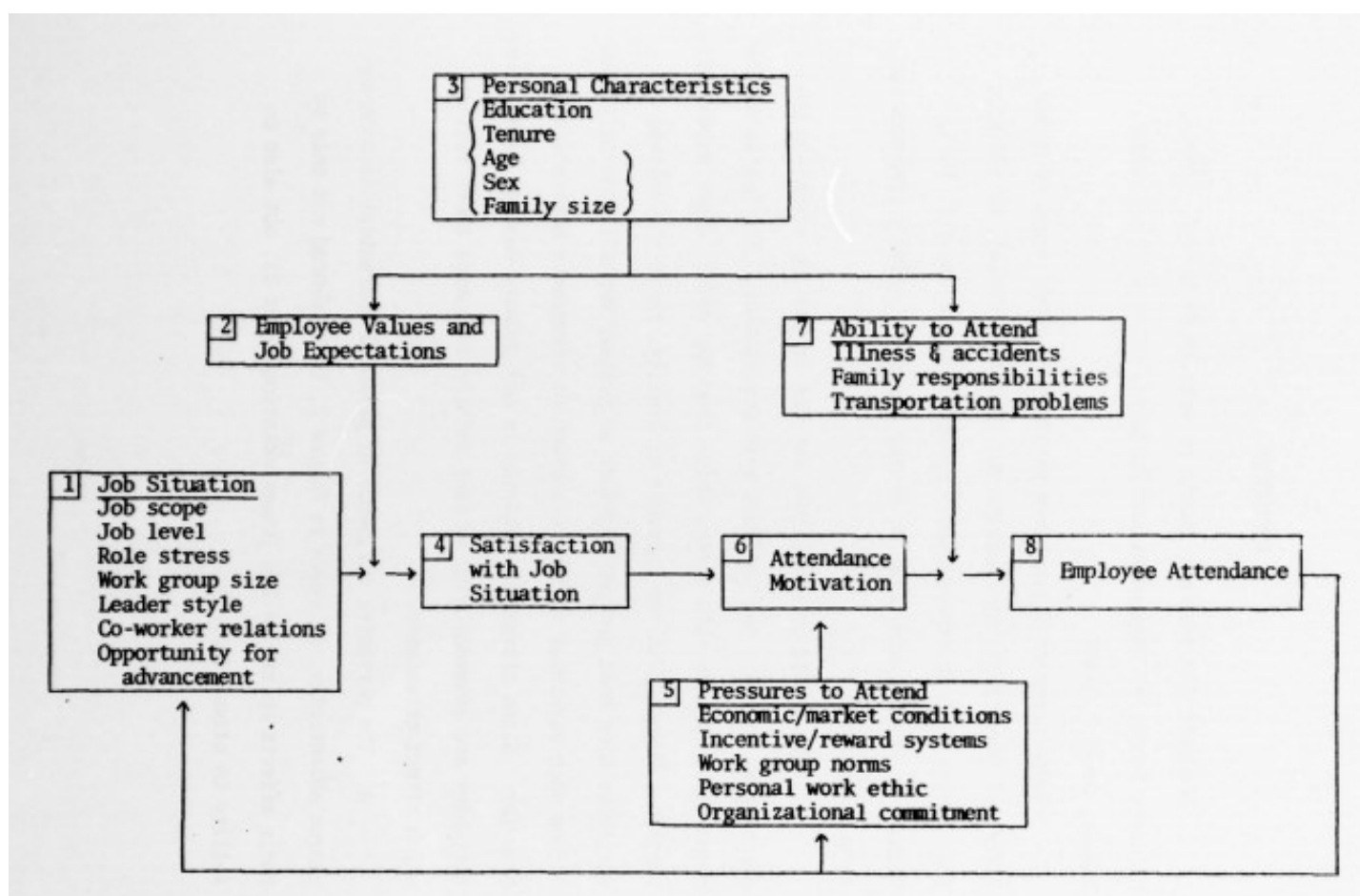

Figure 4: A model of employee attendance (Rhodes and Steers, 1978, p. 53)

\subsubsection{Structure and content of the model}

The structure of the so-called process model is shown in Figure 4. The influencing factors and elements presented there are highlighted below in italics and provided with the corresponding text box number. Essentially, the model serves as an interdis- 
ciplinary explanatory model of the influences on Employee Attendance [8]. The two basic assumptions on which the model is based are, on the one hand, the motivation to take up work and, on the other hand, the opportunity to accomplish that intention, that is to appear at the workplace.

Attendance motivation [6] is identified as the main factor influencing Employee Attendance. It determines the degree of Employ Attendance, provided the employee has the Ability to Attend [7]. The variable Ability to Attend, more precisely its subvariables Illness \& accidents, Family responsibilities, and Transport problems, control the relationship between Attendance Motivation and the current Employee Attendance. The Ability to Attend, on the other hand, is seen as dependent on Personal characteristics [3], under which the attributes Education, Tenure, Age, Sex and Family size are subsumed.

Attendance Motivation is seen as dependent on the Satisfaction with Job situation [4] as well as on internal and external constraints (Pressure to Attend [5]). Satisfaction with Job situation as an intermediary between Job situation [1] and Attendance Motivation is of great importance. Job situation includes the sub-variables Job scope, Job level, Role stress, Workgroup size, Leader style, Co-worker relations, and Opportunity for advancement. Their influence on Satisfaction is controlled by the Employee Values and Job expectations [2], which in turn depend on the aforementioned Personal Characteristics.

Pressure to Attend is understood to be Economic/market conditions, Incentive/reward system, Work group norms, Personal work ethic, and Organizational commitment. They directly influence Attendance Motivation, at least the assumption in the model, in which rather extrinsic motivational factors are addressed.

The dynamic character of the process model is characterized by two overlapping cycles. On the one hand, there is the "fast" cycle Attendance -> Pressure to Attend -> Attendance Motivation -> Attendance, which is to be illustrated by the example of the Reward system: A performance premium is not paid out, this reduces motivation and which in turn leads to more absences, which then leads again to the reduction of performance premiums. The "slow" cycle Attendance -> Job Situation -> Satisfaction -> Attendance Motivation -> Attendance is illustrated by the example of Role stress: Work overload leads to dissatisfaction and thus to a decline in motivation. This leads to outages of some employees, which again leads to further workload for the other employees.

One can ask whether such a relatively simple model can do justice to the complexity of social and human behaviour and whether it can withstand empirical scrutiny. This would go beyond the scope of this work. Here, the ordering and structuring character of an explanatory model is in the foreground on the basis of which the connections in the introduction of primary prevention measures in German general practitioners' surgeries are to be discussed. 


\subsubsection{Applying the model to general practitioners' surgeries}

\section{$\underline{\text { Job Situation [1] }}$}

As explained in subsection 2.2.4, the practicing of one of the measures gives the MFA the possibility to extend her tasks, i.e. the MFA, accompanied by advanced training, can take on the tasks of providing advice on primary prevention. I.e. the $J o b$ Scope may be extended in the sense of job enrichment:" ... the basic theoretical rationale behind such findings is that increasing Job Scope increases the challenge and responsibility experienced by an employee who, in turn, leads to more positive $J o b$ Attitudes (box 4) [Satisfaction with Job Situation]. These attitudes then become translated into an increased desire to participate in what is perceived to be more desirable work activities (box 6) [Attendance Motivation]. Support for this interpretation can be found in Hackman \& Oldham (1976), Indik (1965), and Porter and Lawler (1965)." (Rhodes and Steers, 1978, p. 7)

The peer reviewed activities promoting personal health potentials developed in this section foster the emotional well-being, as will be shown in section 3, so that the Role of Stress is reduced. This applies in particular to measures for movement (sports and fitness), since they are regarded as stress-reducing.

Discussion of Leader Style should be preceded by the fact that in the medical profession there is not a thorough understanding that the leadership of a physician's office is essentially also a management task that is subject to many influences: "Personnel management in medical practices is influenced by the ever-changing economic, technological, legal and social conditions of the practice environment, as well as of specific influences that are based on the structure of the public health system, higher expectations of patients and increased needs of the practitioners. The impact of these environmental factors is increasing, which is particularly true of the pace of technological progress, the trend towards increased qualification of employees, and the ever-increasing legislation in terms of employment, social and tariff law. It is therefore important to include, in addition to the traditional personnel work, important, fundamental success factors of modern personnel management in a doctor's office." (Frodl, 2016 - translated by the author). - Leadership is one of the most effective factors in the goal of health management for general practitioners' surgeries. On the one hand, there is the direct influence of the Leader Style on emotional well-being "... these findings indicate that the behaviour of the leader is a more immediate impact on affective responses to the job situation than on absenteeism itself. "(Rhodes and Steers, 1978 , p. 10) as well as the typical factual situation for physicians' surgeries that work organization and job design are usually determined by the physician. According to Bruch and Kowalevski (2013) the role model function and behaviour in conflict situations play an essential role.

Co-worker Relations - The team in a physician's surgery is easy to grasp, as stated in subsection 2.2.5 does it consist of average 3.5 fulltime MFAs. Failures due to illness have a particularly detrimental effect. The burden on the colleagues can be signifi- 
cantly reduced by means of measures of the primary prevention, so that, in addition to the direct effect on the absence quotas, an indirect effect occurs over the team and thus the operating climate.

\section{Employee Values and Job expectations [2]}

The key aspect of the MFA is that you need to be aware of your responsibility as a responsible person in a health care, to be responsible for personal health, and to be open to primary prevention. The decision to have a helping job shows that "to help others" is an important concern that strongly affects Satisfaction with Job Situation.

This expectation is quite in contrast to the results of the study "Evaluation of job satisfaction of practice staff and general practitioners: an exploratory study" (Goetz, Campbell et al., 2011). In the assessment of ten factors which characterize Job Satisfaction, the mean value for the question of "Recognition for work" is a scale of between 1 (extremely dissatisfied) and 7 (extremely satisfied) only 5.41 (Goetz et al. 2011, Table 2). It is thus clearly below the mean of 5.95 and is the second worst of the ten factors ( $\mathrm{N}=2634$ non-physician staff) following to "Income" with a mean of 4.79.

In the conclusions to the above study is therefore required: “... However, in both cases [physicians and non-physician staff], this was offset by the perception of poor income. The findings of this study will be helpful for further activities to improve the working conditions of GPs and non-physician staff from different perspectives. Nonphysician staff should be supported in their role implicating recognition and appreciation for performance in practice.” (Goetz et al., 2011, p.137)

\section{Personal characteristics [3]}

In the generic model of Rhodes and Steers (1978), Education is called a major influencing factor. This applies in particular to MFAs, whose identity is founded in their professional activities and who are largely oriented on the professional profile of the Employee's Value and Job Expectations: "MFAs are the first point of contact in practice; they receive patients and make appointments. Weighing, measuring, dressing, blood sampling - you take care of the patients. During the examinations, you assist the physicians, document the treatment procedures and record them in the patient's file. MFAs are not only active at the reception and in the examination room, but also work in the laboratory. The use and maintenance of the medical instruments/devices belong to the workday. In the patient talk, you teach about the pre-and post-care and motivate to healthier life. MFAs are the core of the practice: They have organizational skills, ensure a smooth practice and manage administrative tasks (for example, performance reports). In addition, MFAs organize team meetings and organize the use of staff in practice." (Forum Berufsbildung, 2017 - accessed 2017/09/29, translated by the author) 
Regarding the Age factor in the generic model of Rhodes and Steers (1978), the study "How psychosocial factors affect well-being of practice assistants at work in general medical care? A questionnaire survey" of (Goetz, Berger et al., 2015) confirms that age has a significant influence on Satisfaction with Job Situation and general health (Illness and Accidents).

Family size - this factor is important for the MFAs under two aspects: on the one hand, relatively many of the working conditions are part-time jobs. According to Goetz et al. (2011, Table 1) MFAs work per week 26 hours. The full-time quota is calculated according to Gavartina et al. (2013) only $44 \%$ at $\mathrm{N}=586$. This is an effective way to organize family life. On the other hand, the study of Goetz, Berger et al. (2015, Table 4) shows that the "work-privacy conflict" has a major influence on "burnout" and "thinking about early retirement", factors which in turn influence the Satisfaction with Job Situation of the MFAs.

\section{Satisfaction with Job Situation [4]}

The studies by Goetz et al. (2011), Gavartina et al. (2013), and Goetz et al. (2015) confirm the assumptions of the Rhodes and Steers model (1978) on the determinants of the Satisfaction with Job Situation. These studies do not consider the Job Level, the Work Group Size, and the Opportunity for Advancement.

The overall satisfaction of the MFAs is according to Goetz et al. (2011) at a value of 5.95 on the scale $1-7$ at $\mathrm{N}=2634$ as well as 5.74 according to Gavartina et al. (2013) at $\mathrm{N}=586$. But there are also studies, which fear the precarization of the MFAs. E.g. Kathmann and Dingeldey ((2013), who examine the high fluctuation rate and possible exit options.

\section{Pressure to Attend [5]}

„While satisfaction with the job situation thus apparently represents a major influence on attendance motivation, the relationship is indeed not a perfect one. Other factors can be identified which serve to enhance attendance motivation, probably in an additive fashion (Garrison Mochinsky, 1977; Ilgen Hollenback, 1977; Nicholson et al., 1976), these variables are collectively termed here "pressures to attend" and represent the second major influence on the desire to come to work. These pressures may be economic, social, or personal in nature and are represented in figure 4 by box 5" - (Rhodes and Steers, 1978, p. 19)

According to Kathmann and Dingeldey (2013, p. 12), the Economic/Market Conditions are ambiguous: "What is the attractiveness gain or loss of the called occupational field, the results are ambivalent: on the one hand, the increasing number of employees as well as the increased share of older physicians' assistants can be interpreted as a gain in the attractiveness of the profession. On the other hand, there are also indications for a loss of attractiveness in the form of falling training figures and a high exit rate. Likewise, for a reduced attractiveness of the MFA's profession, this 
occupational group fell from the $5^{\text {th }}$ place to $7^{\text {th }}$ place in the ranking of the apprenticeship trades in 2006 (BIBB, 2011)." The current ranking of the apprenticeship is the $6^{\text {th }}$ place $(\mathrm{BIBB}, 2016)$

Incentive/Reward Systems hardly play a role for MFAs. In any case, gratuities on a voluntary basis for dedicated events (e.g. company anniversary, Christmas money, special benefits) would have to be mentioned. Overtime, Sundays and public holidays are usually governed by collective bargaining agreements or labour regulations. From this point of view, promotions of health as a voluntary service are of importance, since they are not formally incentives, but are understood as such.

The working team in general practitioners' surgeries is not large, but this does not affect the importance of Workgroup Norms. In Goetz (2011, Table 4), for example, item "Colleagues and fellow workers" of the 2,634 non-physicians interviewed confirmed the highest degree of satisfaction (6.18 on a scale of 1-7), higher than all other ten satisfaction indicators.

The Personal work ethic often moves in the tension between work-overloading and the "oath of the Hippocrates". The latter idealizes an obligation to optimal treatment in any case, which today is no longer legally tenable, but nevertheless has a considerable influence on the work ethic of the physicians and their staff. In "The hardeveryday life in a general practitioner's office - discrepancies in outpatient medical care" Falk (2010) explains: "Equality and respect for others and their work determine the working environment in practice. Here each patient is taken seriously and accepted according to the obligation to treat, although the practice comprises a total of about 5,000 patients. Approximately 1,000 patients are treated each quarter. For example, the doctor could not accept new patients "since the practice is already fully utilized". However, she insisted on the oath of Hippocrates, which requires the duty of treatment for ethical reasons. "(Falk, 2010, p. 9 - translated by the author).

\section{Attendance Motivation [6]}

MFAs in general practitioners' surgeries are, like many medical and health care professionals, intrinsically motivated. In contrast to this, the MFA's profession has only a low estimate in the society in the sense of Maslow and is associated with precarious working conditions (Kathmann and Dingeldey, 2013), so that according to Herzberg, the hygiene factors are already lacking. Measures to promote health in the general practitioner's surgery represent, in the sense of the incentive-contribution theory, a possibility to promote the performance potential. Depending on the design, the incentives can be both material (e.g., taking over cost of a fitness course) as well as an intangible nature (e.g. recognition of the employee's competence in the field of health promotion).

All the factors presented so far influence the Attendance Motivation. Changes in the influencing factors such as, changes in the work organization, leadership style, etc. 
have a direct or indirect effect on the motivation, and this is one of the two main factors that promote Employee Attendance and absence.

\section{Ability to Attend [7]}

While a high or low Attendance Motivation does not necessarily have the effect that the employee does not come to work, the second main factor - the Ability to Attend is immediate, direct, short-term and inevitable.

The Family Responsibilities must be seen in physicians' surgeries from the point of view that the women's quota is high. Despite changing roles, the family commitments are still common among the women, who tend to care for sick children and relatives and thus have higher absenteeism.

Family Responsibilities and Illness and Accidents have a gatekeeper function. "The most prominent gatekeeper variable is one's health. While sick employees typically do not come to work, it does not necessarily follow that healthy employees will attend. Instead, other factors (e.g., attendance motivation) serve to influence a healthy person's attendance behavior." (Rhodes and Steers, 1978, p.27) This research focuses on the promotion of personal health potentials of MFAs. In the Rhodes and Steers model (1978), one link is Personal Characteristics -> Illness and Accidents -> Employee Attendance. According to the investigations of Goetz et al. (2015), one should regard the "Satisfaction with life" as one of the essential influencing factors that characterize the Personal Characteristics. This, in turn, is influenced by the proposed health promotion measures (primary prevention). In this respect, the work also contributes to the knowledge in addition to the Rhodes and Steers (1978) model.

\section{Employee Attendance [8]}

Since in the Rhodes and Steers (1978) model the health-promoting measures to increase the Employee Attendance also influence Attendance Motivation, primary prevention measures always have a double effect:

- They reduce the disease-related absence

- They improve the motivation and thus increase the motivation-related presence

And the better, the more the preferences of the individual employee are considered and the MFAs are involved in decision-making; because as Goetz et al. (2015) in section "Evaluation of job satisfaction and organizational attributes" state, the involvement of employees in decision-making is the most important organizational factor in the Satisfaction with Job Situation: „Nevertheless 'decision-making' was rated the highest with a mean of $3.95(\mathrm{SD}=0.64)$ showing that practice assistants thought that the practice works as a team concerning decision-making and the development of improvements regarding the quality of care within the practices". 


\subsubsection{A causal model of absenteeism}

In section 3.1.1, the Rhodes and Steers (1978) model of employee attendance was discussed and applied to the occupational field of MFAs in general practitioners' surgeries. Brooke (1986) proposes in his article "Beyond the Steers and Rhodes Models of Employee Attendance" modifications and extensions as they emerge from a literature review, from which a causal model of absenteeism is derived. In this section, the model is examined and discussed in more detail. The following aspects are guiding:

- Unlike Rhodes and Steers (1978), the causal model focuses on absence, rather than attendance, and thus on a topic that is often associated with measuring the success of health management measures. As in the previous section, the path of HR practices in general to the performance of an organization becomes more transparent, the path of organization variables to the performance sub-aspect absence is illustrated here.

- The fulfilment of the psychological contract, as developed in the next section as a success factor for SMEs (Atkinson, 2008), is confirmed from the perspective of the author with this model.

- The aim of this research is, inter alia, to identify measures to promote personal health potential. The next section examines those measures that have been proved to reduce absenteeism. As the main effect of the introduction of measures of primary prevention foremost improving the health status of employees is expected. But as with any intervention, side-effects, repercussions, and long-range effects must be considered, which can additionally increase the expected results, but also jeopardize them. The relationships of the causal model of absenteeism are therefore included in the cross-impact analysis for the implementation of health management.

- The causal model of absenteeism helped to develop and prepare the structured interviews with general practitioners to reflect the results of the employee survey on the priority concept.

- The causal model is explained with reference to work-scientific studies of the occupational field of MFAs. The results of them are examined and compared with the model variables.

\subsubsection{Structure and content of the model}

The following figure shows the model proposed by Brooke (1986, p. 350); the light blue background was highlighted by the author with reference to the "HR practices psychological contract - organizational performance linkage framework" (Katou, 2013) especially in SMEs (Atkinson, 2008). Terms used in the model are written in italics below. Compared to the Rhodes and Steers (1978) model, it first stands out that the target is now Absenteeism and not attendance. Absenteeism is determined by exogenous variables such as Kinship Responsibility and endogenous variables such as Health Status. Endogenous variables are affected by other endogenous variables, 
such as Satisfaction influences Health Status, or are affected by exogenous variables, such as Role Overload on Health Status. In the model, all variables that are not input from another variable are considered exogenous. They are all grouped on the left side of the picture.

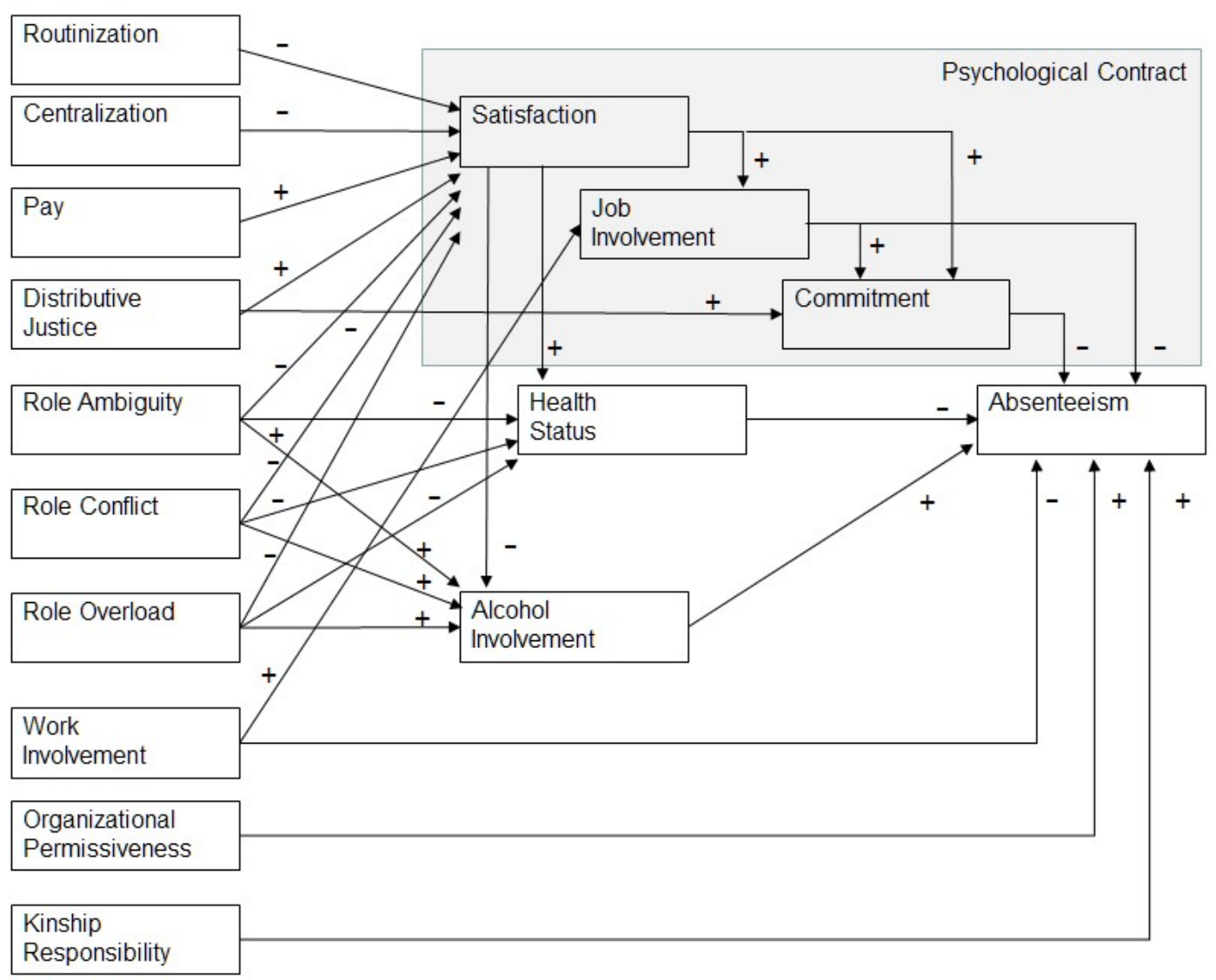

Figure 5: Causal model of absenteeism and psychological contract (source: Brooke (1986, p. 350)-extended by the author)

The relationships between variables are represented as arrows in a path diagram. The arrow means strengthening the source (arrow start) leads to the strengthening of the target (arrowhead). Thus, more Pay leads to more Satisfaction. So, an arrow marked with "+" means: The more ... the more ... and the less ... the less (direct effect). Arrows marked with "-" have an inverse direction. If there is less Role Overload, the Satisfaction increase. Conversely, more Role Overload leads to less Satisfaction. In the case of opposing relationships, the less ... the more ... and the more ... the less (indirect effect).

"The ordering of exogenous and endogenous variables, and the direct and indirect effects depicted in the path diagram reflect an integration of the predominant empirical relationships regarding each concept which have been reported in the literature." (Brooke, 1986, p. 348). Compared to the Rhodes and Steers (1978) model, all variables are operationalized in questionnaire form based on work by Price (1972), Price (1977) and Price and Mueller (1981), Price and Mueller (1985). In addition to the clarification of concepts by variables further variables were added that were relevant, 
but not yet represented: Job Involvement, Distributive Justice and employee Involvement with alcohol.

The endogenous variables Satisfaction, Job Involvement, and Commitment form part of the content of the psychological contract (Rosseau, 1989; Atkinson, 2008; Katou, 2013). This emphasis highlights the dual impact of primary prevention measures, the main theme of this research. On the one hand, these measures directly affect the Health status and on the other hand, these measures influence the variable Satisfaction of the psychological contract, which in turn also affects the Health status.

It also highlights the central importance of the psychological contract. All presented exogenous variables, except for Organizational Permissiveness and Kinship Responsibility, affect the content of the psychological contract. As in the HR practices - performance framework of the next section (Figure 7, it forms quasi the core, the "heart" or the "motor" of the causal model. According to the author, the variables Organizational Permissiveness and Kinship Responsibility also influence the variables of the psychological contract in micro-enterprises, relationships which are not contained in the original model.

\subsubsection{Applying the model to general practitioners' surgeries}

In the following, the exogenous model variables and the related impact relationships regarding their significance for MFAs are discussed.

\section{$\underline{\text { Routinization }}$}

Routinization is the degree to which the cycle of tasks in a job is repetitive (Perrow, 1967 - cited in Brooke, 1986). In the study by Gavartina et al. (2013), this exogenous variable is most likely associated with "Amount of variety in job". With an average of 5.49 on a scale of 1 to 7, this factor ranks second for the MFAs. The factor for $J o b$ Satisfaction, which can also be associated with Routinization, is "freedom of working method". At the same scale, it reaches a value of 5.20 (rank 6), so that in the overall picture, Routinization should not be a disabling factor for Job Satisfaction. This is confirmed in a comparable study with a similar result by Goetz et al. (2013)

\section{Centralization}

To apply this variable to an investigation of MFAs' workplace seems at first inappropriate, as work general practitioners' surgeries are, as stated, sole proprietorships and unaffected by centralization considerations. In fact, another notion of concept is underlined: "Centralization is the degree to which worker power is differentially distributed within an organization" (Hall, 1982 - cited in Brooke, 1986, p. 351). The concentration of all organizational power in a single individual would reflect a maximum degree of centralization. "In the study by Gavartina et al. (2013), this exogenous variable is most closely linked to the "Amount of responsibility". With an average of 5.38 on a scale of 1 to 7, this factor ranks third in the MFAs. This is confirmed in a comparable study with a similar result by Goetz et al. (2013). 
$\underline{P a y}$

For MFAs, the biggest downside seems to be here. This impression runs through all studies. In Gavartina et al. (2013), the factor "Income" is by far the poorest factor influencing Job Satisfaction (3.89 on a scale of 1 to 7 - 9th place). This is confirmed in a comparable study with a similar result by Goetz et al. (2013). Further confirmations of this relationship for the occupational field MFA (especially in medical practices) can be found in Höhl (2013), Kathmann and Dingeldey (2013, 2014), so that low pay can be regarded as the main cause of Dissatisfaction in the job.

\section{Distributive Justice}

Distributive Justice is the degree to which rewards and punishments are related to performance inputs into the organization (Homans, 1961 - cited in Brooke, 1986, p. 352). This concept gives the degree to which they are responsible for their contributions and efforts on behalf of the organization. The model hypothesizes that Distributive Justice has direct effects on Satisfaction and Commitment, and indirect effects on absenteeism through these intervening variables (Brooke, 1986). There are no indications of these relationships in the studies on Job Satisfaction, Involvement and Commitment of MFAs. Although an online survey of 596 MFAs by Höhl (2013) suggests that in $23 \%$ of MFAs with under-tariff pay and another $20 \%$ with a lower-pay subgroup, there are inconsistencies in Distributive Justice, but that is at best the condition of the variable and not its impact on Satisfaction or Commitment.

Kathmann and Dingeldey (2014, p. 242) states in addition: "Research results indicate that employees in the labour market [MFAs] are not perceived as being at risk. Only low pay is considered as a problem. - Since it is to be assumed that, before it comes to the change of employer or career change, the Satisfaction and the Commitment significantly decrease, the results of the evaluated research project can certainly be seen as confirmation of these relationships in the causal model of absenteeism.

\section{$\underline{\text { Role Ambiguity }}$}

Role Ambiguity is the degree to which role expectations are unclear (Rizzo, House, \& Lirtzman, 1970; Locke, 1976 - cited in Brooke, 1986, p. 352). In Goetz et al. (2013), this exogenous variable was examined in addition to other organizational factors with the item "Responsibilities within the practice team are clear". In 1,145 respondents, this factor was rated 4.26 on a 1-5 scale (ranked 1 of 4 characteristics). An indication that the MFAs have little ambiguity regarding role expectations.

This is confirmed by a survey result by Goetz et al. (2015) confirming "role-clarity" as the second most important psychosocial factor, which is considered by the MFAs as essentially fulfilled. ( 81.6 on a scale of $0-100, \mathrm{n}=586$ ). The negative correlation shown in the causal model is also confirmed indirectly. Higher "role-clarity" means less Role Ambiguity and thus higher Job Satisfaction. 


\section{$\underline{\text { Role Conflict }}$}

The influence of Role Conflict on Health Status is described in Goetz et al. (2015) by finding a negative correlation between "role-conflict" and "burnout" as components of Health Status in the MFA survey. Similarly, the negative correlation between Role conflict and Job Satisfaction in the causal model is underpinned.

\section{$\underline{\text { Role Overload }}$}

The "Hours of work" most closely associated with this variable is found in the study by Gavartina et al. (2013) with 5.34 in midfield. This is confirmed in a comparable study with a similar result by Goetz et al. (2013).

The influence of Role Overload on Health Status is described in Goetz et al. (2015) supported by showing a positive correlation between "cognitive stress symptoms" as a component of Role Overload and "Burnout" as components of Health Status. Likewise, the negative correlation between Role Overload and Job Satisfaction in the causal model is underpinned. Also, the factor "quantitative demands", as a sub-item of Role Overload, is confirmed by this study by a negative correlation.

\section{$\underline{\text { Work Involvement }}$}

This exogenous variable primarily affects the Job Involvement and directly Absenteeism. In the studies by Gavartina et al (2013) and Goetz et al. (2013) this variable corresponds to the factor "Opportunity to use abilities". The average value of 5.26 resp. 5.77 leads to rank 4 or 5 . This can be seen as confirmation that the MFAs are employed according to their abilities. This is also in line with the BIBB study (2012) on job description and job design for MFAs, which will be explained later.

Goetz et al. (2015) used the Copenhagen Psychosocial Questionnaire (COPSOQ) for evaluating psychosocial health and work. The 586 MFAs surveyed rated the "Meaning of work" as the second most important psychosocial factor, which is crucial for motivation and positively correlated with Job Satisfaction and Job Involvement. Brooke (1986) citing Kanungo (1982) presents "Work Involvement is a normative belief in the centrality of the work role in one's life". Related to this point of view, the COPSOQ-item "Satisfaction with life", which is also correlated with Job Satisfaction is relevant. This factor as a component of Work Involvement also has a correlation to the endogenous variable Health Status, which is thus confirmed as well as the relation Job Satisfaction and Health Status.

\section{Organizational Permissiveness}

This exogenous variable is explained in Brooke (1986) as follows: Organizational Permissiveness is the degree to which Absenteeism is accepted by an organization (Parsons, 1956). The central idea of this concept is "frequent absence without consequence" (Oberman \& Rainer, 1983). An organization or subunit in which members are able to take unscheduled days off easily, or in which numerous casual absences 
result in little or no apparent adverse consequences would be highly permissive toward absenteeism (see Morgan \& Herman, 1976; Dalton \& Perry, 1981). This concept seems to be implied by the "leader style," "incentive/reward system," and "work group norms" variables of the Rhodes and Steers (1978) model.

There are no explicit references to this variable and its relation to others in the available studies on the MFA occupational area. If one refers to the "Leader style" hint, then Goetz et al. (2015) derive a relatively high importance of the "Quality of leadership" (65.9 on a 0-100 scale) and recognize a connection to Job Satisfaction. However, it can be assumed that the management of Absenteeism by the physician as owner manager is only a partial aspect of the "Quality of leadership".

\section{$\underline{\text { Kinship responsibility }}$}

The primary focus of Kinship Responsibility is on parental responsibility for the care of children or other dependent relatives who reside within the local area (Brooke 1986, p. 353). In the study by Goetz et al. (2015), the psychosocial factor "WorkPrivacy-Conflict" does not attach as much importance to the MFAs as a job demand. However, the statistical evaluation confirms a strong correlation to "Burnout" and thus a negative correlation to the Health Status. It is interesting to note that this factor is given nearly twice as much importance by full-time MFAs as part-time MFAs (35.7 vs. 18.0).

\section{Other factors influencing Job Satisfaction and Health Status}

In the study by Gavartina et al. (2013) on the occupational field MFA, the German version of the "Warr-Cook-Wall (WCW) job satisfaction scale" developed by Warr, Cook et al. (1979) was used to determine influencing factors. Six of the nine influencing factors are considered in the causal model of absenteeism (Brooke, 1986). The missing influence factor "Colleagues and fellow workers" is mentioned as the most important factor in terms of satisfaction. In addition to income, the factor "Recognition for work" most likely contributes to dissatisfaction. This should not be seen unrelated to leadership abilities in general practitioners' surgeries, especially as this factor, according to regression analysis in the exploration study by Goetz et al. (2011) is the strongest contributor to job satisfaction (followed by "Colleagues and fellow workers." Also, the factor "Physical working conditions," ranked 7th out of 9, should be motivating for leading a general practitioner's surgery.

The study by Goetz et al. (2015), which is essentially based on the job demandscontrol model, shows the psychosocial factors "Emotional demands" and "Demands for hiding emotions" as further factors that are of importance for MFAs and are not considered in the causal model. Both are negatively correlated with the Health Status and the Job Satisfaction, but from the perspective of the MFAs they are not considered as relevant (47.9 and 44.9, respectively). 


\subsubsection{Peer reviewed activities promoting personal health potentials}

The causal model of absenteeism presented in the previous subsection (Brooke, 1986) as a revised Rhodes and Steers model (1978) shows the health status as one of the most important factors influencing involuntary absenteeism. "Researchers have endorsed the direct effects of health status on absenteeism (Muchinsky, 1977). However, illness is widely recognized as the cause of absenteeism (Paringer, 1983, Hedges, 1973, 1975, 1977), accounting for from one-half to two-thirds of all employee absence (Miner \& Brewer, 1976) "- Brooke, 1986, p. 354. While in the causal model of absenteeism satisfaction, role ambiguity, role conflicts and role overload are identified and documented as endogenous determinants, this subsection deals with (exogenous) measures and activities that promote personal health potential and help prevent illnesses in the workplace. For them, it is examined which peer reviewed articles document a reduction of absence. At the same time, they serve as a basis for interviewing employees regarding their preferences.

This relationship is the focus of the first research question:

1. What research studies indicate a strong link between measures of health promotion and a direct result in reduction of absenteeism?

The area of health management is broad and there are many different studies, with a focus on different branches, diseases, organisations, countries and people - 26,332 results were found for literature search with the key word "health management".

For a systematic review, sources from England and Germany were used. The resources for the overview in England have been the Ebsco database and additional for British doctoral theses EThOS - Electronic theses online service.

For German theses, the German National library (DNB) was used which has an additional service for the research of doctoral theses DissOnline; including all theses from Germany and Switzerland which are available online. The first database was Ebsco followed by the DNB, EThOS and DissOnline.

The research used different kinds of sources, with an emphasis on peer reviewed academic journals or articles:

1. Academic articles, theses and papers

2. Academic textbooks

3. Specialists' conferences and interviews with experts

The search of ,health management” in all databases provided the starting point. The focus was on studies that are positioned within the service sector. After this broad search, the search was narrowed down to disease prevention at the workplace.

The search for constraints on SMEs and the exclusion of big companies was one option. During the research, several options were used. If necessary and meaningful, 
the region was restricted. The focus was on general practitioners' surgeries within Germany, if there were insufficient results the research was focused on SMEs in general.

\subsubsection{Search area: Disease prevention at the workplace}

An important measure for the level of health management is the number of absent days. Therefore, the next step in the literature search was to find data on the absent days in German general practitioners' surgeries or SMEs including information about what disease caused them. The need of efficient activities for improving the health management is important for SMEs because they must use the resources which are most effective.

The health insurance system in Germany provides yearly figures about absent days. In Germany 69,820,682 people are in the national health insurance (BMG, 2011)

The BARMER GEK, one of the national health insurance companies, has published a study including absent days in 2010. The "Health report 2010" (Grobe, Dörning et al., 2011) gives a German wide overview about absent days linked with the kind of disorder and it is broken down on every federal state. It has no figure about the size of the companies the employees work for.

Additional the IGA report (Sockoll, Kramer et al., 2006) which is a governmental study of the scientific evidence from 2000 to 2006 concerning healthcare management in companies, gave a detailed view on the reasons for absent days.

The "Health report 2010" (Grobe, Dörning et al., 2011) defines four main reasons for absent days:

\begin{tabular}{|l|l|}
\hline Disorder & $\begin{array}{l}\text { Absent days (2010 per employee } \\
\text { in average) }\end{array}$ \\
\hline Musculoskeletal system disorders & 3.34 \\
\hline Mental disorders & 2.44 \\
\hline Diseases of the respiratory system & 2.00 \\
\hline Injury & 2.00 \\
\hline Total & $\mathbf{9 . 7 8}$ \\
\hline
\end{tabular}

Table 1: Absent days of German employees (Grobe, Dörning et al., 2011)

The average absent days and the recommendations from IGA report (Sockoll, Kramer et al., 2006) has been the basis for the next steps in the literature search. The IGA report (Sockoll, Kramer et al., 2006) identified several interventions at the workplace, and proved that they are useful to reduce absent days. Disease prevention at the workplace is the central control parameter for reducing and steering the absent days. This data has the disadvantage that it is not divided into different sectors or branches like industry or service.

For a more conclusive research the different areas of disease were structured following the IGA report (Sockoll, Kramer et al., 2006). Combined with "Health report 2010" (Grobe, Dörning et al., 2011) about the absent days, there were five main top- 
ics on which the further literature search was focused on. Every topic had its own sub-items. These topics cover the whole disease at the workplace. The goal of health management is to prevent the possible disease, therefore studies with a focus on aftercare have only been considered if there was also an influence on the prevention. The sequence of the points was based on the number of absent days caused in average at this area by (Grobe, Dörning et al., 2011). It starts with the most and goes down to less. The last point is the general health and well-being at the workplace and related disease. The absent days are structured after ICD10 code, which is used by the health insurance in Germany.

1. How to prevent musculoskeletal disorders?

2. How to prevent mental disorders at the workplace?

3. How to prevent diseases of the respiratory system at the workplace?

4. How to prevent injury at the workplace?

5. How to promote general health and well-being at the workplace?

For each of the five main topics, Appendix 2 contains the peer-reviewed articles found in the literature review as a tabular overview. The results were given a relevance note and a brief reference to the potential benefits of this research. In the following, only the articles that have been rated as "relevant" or "partly relevant" are discussed.

\subsubsection{How to prevent musculoskeletal disorders?}

The study "Workplace interventions to prevent musculoskeletal and visual symptoms and disorders among computer users: a systematic review" (Brewer et al., 2006) found hints that interventions in the office could help to reduce absent days caused by musculoskeletal disease: "An important message to all stakeholders is that the current state of the peer reviewed literature provides relatively few high quality studies of the effects of office ergonomic interventions on musculoskeletal or visual health.“ (Brewer, Van Eerd et al., 2006)

The article "How we prevent musculoskeletal disorders in the workplace" (Melhorn and Gardner, 2004) gives an example for the caused costs for the national economy of the USA. It is not focused on SME and not in the defined region. „In 1999, a National Academy of Sciences study found total costs to be more than US dollar 1 trillion per year and concluded that effective prevention of workplace pain through active intervention is not only possible, but results in a significant cost saving for the employer, while reducing the disability experienced by the employee." (Melhorn and Gardner, 2004)

The governmental study of the IGA (Bräunig, Haupt et al., 2015) includes systematic research for the area general promotion of general health and well-being at the work place. Abraham and Graham-Rowe ((2009) found out that programs for more physical activities which are developed after 1997 and include pedometer help to improve health management. The focus on movement with a pedometer is four times as effec- 
tive as other activities, which was confirmed by the systematic research of Dugdill et al. (2008).

In his journal article "Integration of Short Bouts of Physical Activity into Organizational Routine: A Systematic Review of the Literature." (Barr-Anderson, 2011) found out that "short activity-bouts" during work time brings a moderate but significant effect.

\subsubsection{How to prevent mental disorders at the workplace?}

Bräunig, Haupt et al. (2015) realized that in the last years the area of mental disorders is the fasted growing disease. There is a lot of attention in the articles, but this is not equally reflected in studies or peer reviewed papers.

The "Improving responses to depression and related disorders: Evaluation of an innovative, general, mental health care workers training program." (Graham, Julian et al., 2010) found some moderate evidence for the effect program for mental health prevention, but it is focused on Australia and it could not be identified if the prevention would fit the German job profiles.

There were no articles, which consider the linkages between mental disorder and prevention. At a German congress of occupational medicines, the theme mental disorder burnout was discussed very intensively. The search in Ebsco showed as a result 116 articles for "prevent burnout ", but they are focused on job profiles like nurses or teachers, and therefore they are not directly useful for the service sector in general. In terms of burnout, the professional situation of teachers or nurses is not comparable to MFAs. Teachers have to deal almost continuously with a large, present number of students, MFAs are usually dealing with individual patients. Nurses perform heavy physical work (e.g. patients into another beds) and usually have shift work (day and night duty).

\subsubsection{How to prevent diseases of the respiratory system at the workplace?}

The area of respiratory provides extremely many studies. It is a well-explored area of human medicine. The number of results which are linked with occupational medicine was not very high.

The article "Cost-benefit analysis in occupational health: a comparison of intervention scenarios for occupational asthma and rhinitis among bakery workers." (Meijster, van Duuren-Stuurman et al., 2011) is focused on the bakery sector but a part of it is how to develop and implement strategies for the health management which is an enlightening link to the BSC, "Translating Strategy into action" (Kaplan and Norton, 1996). This result fitted in the area of performance measure for further research.

„This study provides important insights for developing effective intervention strategies in the field of occupational medicine. Use of a model-based approach enables 
investigation of those parameters most likely to impact on the effectiveness and costs of interventions for work related diseases. Our case study highlights the importance of considering different perspectives (of employers, society and employees) in assessing and sharing the costs and benefits of interventions.“ (Meijster, van DuurenStuurman et al., 2011, p. 745).

However, there were no results for the service sector and the prevention of illness of the respiratory system.

\subsubsection{How to prevent accident and injury at the workplace?}

The German law has detailed information how accident and injury could be prevented at the workplace. This German law for occupational medicines, security engineers, occupational health and safety practitioner was issued 1973 and is the framework for what organisations in each size must do to avoid occupational accidents.

Additional to the law there is a system of seventeen professional associations in Germany, the federal work insurance (BG). These (governmental) insurances under public law provide for each of the branch services concentrated on accident prevention. Every organisation has to insure their employees in one of these BGs.

They do intensive research and very broad activities in the area of accident prevention and every company has access to the support of the BG.

\subsubsection{How to promote general health and well-being at the workplace?}

This is a reservoir for several themes concerning health. There is a connection to the workplace, but it was difficult to narrow it down. Here the sub-items suggested in the IGA report were used (Sockoll, Kramer et al., 2006) combined with the top reasons for absenteeism (Grobe, Dörning et al., 2011).

These sub-items were broad; the research gave an overview of the existing abstracts and showed studies, which proved the influence from promotion of general health management to the absent rate of employees. The study "Health-related behaviours and sickness absence from work" (Laaksonen, Piha et al., 2009), which is a result for the next search point physical activity, evaluates the connection between absent days and health-related behaviours and found out that decreasing of smoking and relative weight can help to provide important advantages in work. This can lead to reduce sickness and reduce absent days.

\subsection{Physical activity}

The search for 'physical' returned no result. But there is a link between sport and overweight, which has an influence on the absent days as documented by (Laaksonen, Piha et al., 2009) but this connection is only indirect. To find relevant results the search word 'physical activity' was replaced by 'sport' and by this way twelve relevant articles has been found. 
One of these twelve articles in the search results was linked with occupational medicine and the health management in an organisation: "How do sports and dietary restraint relate to overweight-associated absenteeism?" - (van Strien and Koenders, 2010). The other eleven articles were not related to health management.

In the article "Health-related behaviours and sickness absence from work" (Laaksonen, Piha et al., 2009) describe that overweight, behind smoking is the second most factor for absent days. Therefore, a study, which proves that health management programs can lead to reduced weight, seemed to be insightful.

In their study "How do physical activity, sports, and dietary restraint relate to overweight-associated absenteeism?" (van Strien and Koenders, 2010) strengthened this link. Health management programs, which focus on promoting the physical activities, combined with other actions could reduce overweight of their employees.

"Physical activity and sports acted as moderator variables in that they both attenuated the positive relationship between overweight and/or obesity and absenteeism. Restrained eating also moderated the relationship between overweight and absenteeism. Remarkably, the degree of overweight was more strongly related to augmented absenteeism in people with higher levels of restrained eating." (van Strien and Koenders, 2010)

Remarkable of this study was the result, that there is no mandatory influence from physical activities to weight loss and reduced absent days: "To reduce absenteeism in overweight employees, it may be beneficial to focus on stimulating physical activity and not on interventions aimed at promoting dieting (restrained eating)." (van Strien and Koenders, 2010).

The points physical activities, nutrition and weight control were strongly cross-linked with each other and there were few studies, which have analysed these correlations combined with occupational medicine or health management.

\subsection{Nutrition and weight control}

The identified studies did not give good information about a connection between weight losses and reduced absent days.

In the study "Worksite environmental interventions for obesity control: an overview" (Lemon and Pratt, 2010) evaluated the impact from psychosocial work stressors on the body mass index.

In these results, no study evaluates the linkage between healthy nutrition and the absenteeism rate in an organisation. The study „Smoking and high relative weights were most strongly associated with sickness absence "(Laaksonen et al., 2009) shows that being overweight has a comparable bad influence on absent days like smoking.

The study „Effectiveness and economic benefits of workplace health promotion and prevention" (Sockoll, Kramer et al., 2006) is not in the result list of this point. How- 
ever, it provides worthwhile findings concerning nutrition. According to World Health Organisation (Engbers, 2007) workplace programs for improving eating habits are generally accompanied by positive effects, even though they are rather moderate. Nevertheless, they can influence the employee's consumption of fruit, vegetables, and fat, as well as their intake of dietary fibres significantly as shown in controlled studies. Products and other informational strategies can encourage the sale and consumption of healthier food and thus a healthier food selection of the employees during working hours. Matson-Koffman, Brownstein, Neiner and Greaney (2005) state that there is strong evidence in this context.

On the other hand, the results of studies on the effectiveness of incentives to improve eating behaviour are contradictory. If they are implemented in addition to informational strategies, they might be more effective. "Hardly any intervention was tested for its effect on physiological outcomes, which would be much more convincing. Therefore, the authors of the two latter reviews see an urgent need for conducting better evaluation studies, as well as developing innovative prevention methods for modifying the work environment beyond the simple strategies used up to now." (Sockoll, Kramer et al., 2006)

In the "Worksite-based weight loss programs: a systematic review of recent literature." Benedict and Arterburn (2008) found that moderate short-term weight reduction can be achieved.

\subsection{Smoking cessation and tobacco control}

The first step of the literature search showed 14.152 peer reviewed articles. If the search is restricted to a reference to occupational medicine, seventy articles remain.

In their study "Occupational Risks Associated with Cigarette Smoking: A Prospective Study" (Ryan, Zwerling et al., 1992) evaluated the linkage between smoking employees and a negative impact to the organisation. "...Our study shows that cigarette smoking is associated with adverse employment outcomes after controlling for a number of possible confounders ..." (Ryan, Zwerling et al., 1992).

The study "Health-related behaviours and sickness absence from work" by (Ryan, Zwerling et al., 1992) shows that smoking and a high weight are often the first and second reasons for sickness absence. That means that programs, which lead to less smoking employees, can reduce the absence rate. "Smoking and high relative weight were most strongly associated with sickness absence, while the associations of other studied health-related behaviours were weaker. The associations were stronger for medically confirmed sickness absence spells for which heavy smoking and obesity more than doubled the risk of sickness absence in men and nearly doubled in women ... "(Laaksonen et al., 2009, p. 840).

In a Cochrane study Cahill et al. ((2015) found out those programs against nicotine work better if they are in a group or with a professional face-to-face meeting. Self- 
study materials do not work. No advantage through a competition but through teamwork.

\subsection{Programs for alcohol prevention}

The database search in Ebsco returned three results.

A study from Sweden (Holmqvist, Hermansson et al., 2008) give an insight into the effectiveness of health management; it could be improved through making existing structures more effective „... Both the physicians (72\%) and nurses (90\%) said that the knowledge about counselling techniques to use when alcohol-related symptoms are evident was the most important facilitator to increased intervention activity. Conclusions: OHS professionals usually discuss alcohol-related issues with their patients. Nonetheless, they are interested in gaining further education and knowledge in this respect. The study results indicate that OHS is an important setting for alcohol prevention"

The study „Preventing high-risk drinking in youth in the workplace: A web-based normative feedback program“ (Doumas and Hannah, 2008) found out that a webbased feedback program could help young working adults prevent drinking.

The study „Does job satisfaction mediate the relationships between work environment stressors and employee problem drinking" (Berger, Sedivy et al., 2008) analyses the link between alcohol prevention, the workplace and employees drinking problem. ,... Several study variables of interest were found to be associated significantly and directly with an employee's problem drinking status. Implications for workplace alcohol prevention are discussed“" - (Berger, Sedivy et al., 2008)

\subsection{Programs against infectious diseases}

There are two kinds of prevention before infectious diseases, the prevention for companies, which have no contact to infected people, and organisations like hospitals. Some hospitals have special care departments for high-risk disease like Ebola. The health management approach with prevention is focused on the normal day-to-day business. Generally, it is not fixed that there is a higher risk for employees in the medical sector. Several general practitioners' surgeries do not have more contact with people with an infectious disease. Some of them have more contact to clients, which have an infectious disease. However, around infectious disease prevention the German Federal Centre for Health Education (BZgA) has its focus on health education and health promotion. Following the BZgA there are two principal areas, the hygiene, and vaccines.

The database search in Ebsco for 'vaccines' returned nineteen articles. Six articles from them are not in the table (Appendix 2), because they were newsletters published in journals, which were not useable in this literature search. 
Following the study "Occupational vaccination of health care workers: Uptake, attitudes and potential solutions" (Institut für betriebswirtschaftliche Analysen, 2012), vaccines and especially the regular flu vaccines are important for health management and specially for people who work in the health sector. "Improving influenza immunization uptake in healthcare workers" (Preece, 2012) supports this opinion with a focus on the flu vaccine. Both do not separate employees in hospital and general practitioners' surgeries.

\subsubsection{Conclusion to literature search}

The findings on the search area "Disease prevention" of this subsection provide an evidence-based approach to answer the first research question for the specific measures to be investigated in primary prevention for MFAs:

1. What research studies indicate a strong link between measures of health promotion and a direct result in reduction of absenteeism?

Although the studies found provide some valuable information on the design and the challenges of health management, it is remarkable that no specific studies are available on the employment situation of an economically not insignificant profession, the MFAs (417,000 persons cf. 2.2.1). Regarding the guiding model for multi-criteria decision-making, the first steps, highlighted in Figure 6, with the literature search were completed.

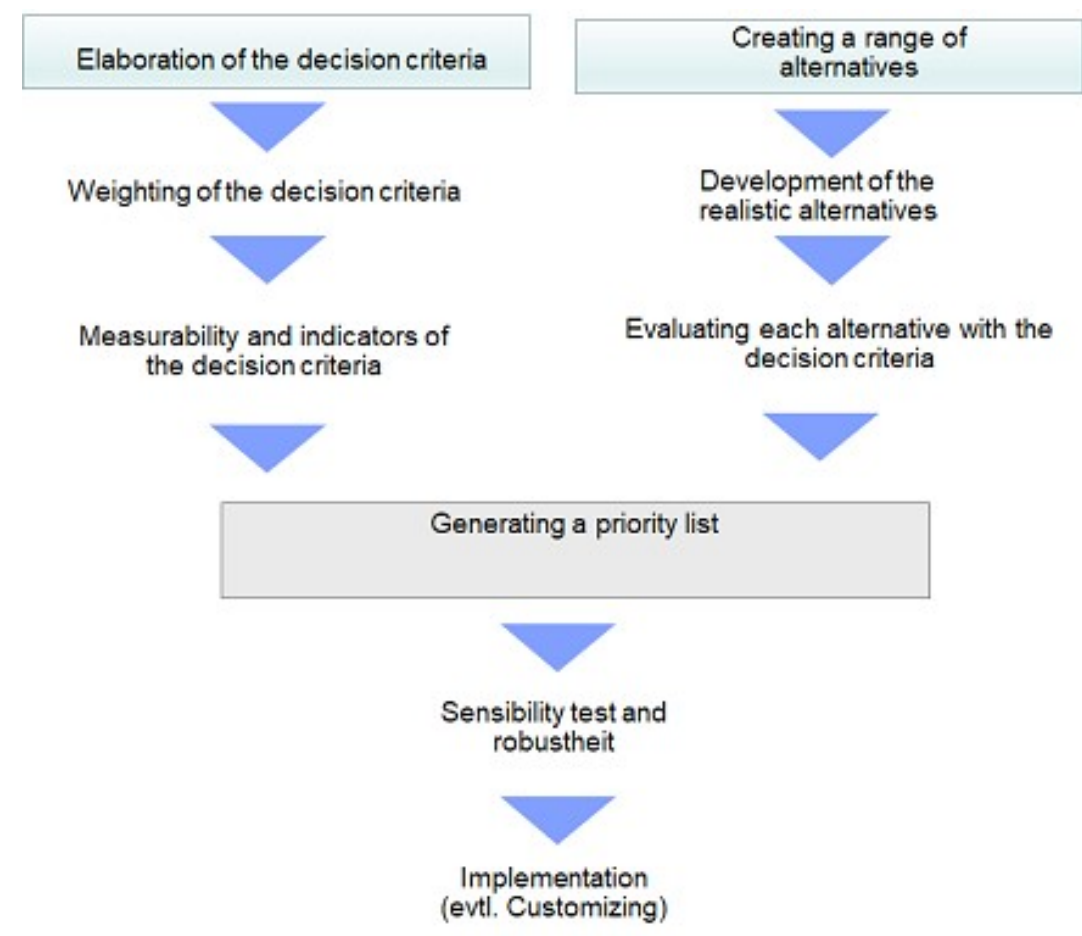

Figure 6: Literature search as starting point of the multicriteria decision-making model 
The next chapter will explore the different methodologies that might be considered as suitable and workable for the investigation and the design of a questionnaire for activities of primary prevention.

\subsection{A framework to increase performance in micro-enterprises}

Activities to promote personal health potentials, which are the focus of this work, can be classified in a comprehensive understanding as measures of human resource management (HRM). HRM itself can be understood in the broadest sense as "all those activities associated with the management of people in firms [organizations] ... HUMAN resource management (HRM), the management of work and people towards desired ends, is a fundamental activity in any organization in which human beings are employed. It is not something whose existence needs to be elaborately justified: HRM is an inevitable consequence of starting and growing an organization. While there is a myriad of variations in the ideologies, styles, and managerial resources engaged, HRM happens in some form or other." (Boxall, Purcell et al., 2008, p. 1). However, there is no real consensus about the practices of a coherent HRM system, and certainly not a unified understanding or theory about the relationships between HRM and performance. Boselie, Dietz et al. (2005, p. 5) point out in their article "Commonalities and contradictions in research on human resource management and performance" that HRM is "... in its broadest sense, concerned with the selections that organizations make from among the myriad of policies, practices and structures for managing employees ". In the narrower sense, "HRM is conceptualized in the form of carefully designed combinations of methods for improving organizational effectiveness and hence better performance outcomes, as with Wright and McMahan's definition (1992, p. 298): " the planned HR deployments and activities intended to enable [an organization] to achieve its goals "(see also Delery and Doty (1996, p. 805))."

In the following, the link between primary prevention measures as a specific HR practice and an increase in general practitioners' surgeries performance as a specific form of organizational capacity against this theoretical background is examined.

Common to all theories is that they assume that HR practices increase performance and have focused on this relationship in recent decades like Huselid (1995), Becker and Gerhart (1996), and Wright and Boswell (2002). Likewise, it is assumed that "the idea that effect of HRM on organizational performance mainly goes through people." (Peccei et al., 2013, p. 16). Differences arise from the explanatory approaches that are attempted to make the so-called "Black box" (Boxall, Purcell et al., 2008) to a "white box" - or in other words: "Unlocking the Black Box" (Messersmith, Patel et al., 2012). In explaining how the black box works, different attitudes and their influence on behaviour are examined. The consideration of the respective environment and organizational structures also takes place in different ways. Initially committed to resource oriented HRM - e. g. (Arthur, 1994), (Wood and de Menezes, 1998), (Paauwe and Richardson, 1997), (Patterson, West et al., 
1997) - job satisfaction, motivation, and trust were increasingly seen as key determinants (Guest, 1998) (Boxall and Macky, 2007), as well as for instance involvement (Wood and Wall, 2007). Additional approaches also consider that happiness and the closely related health and well-being have a significant impact on behaviour and performance (Atkinson and Hall, 2011).

\subsubsection{Resource-based approaches for micro-enterprises}

In a first step, the author sought to decide which theoretical approach would be most likely to be used to justify, investigate and explain primary prevention as part of health management as an HR-practice and its influence on performance. Before doing so, some important peculiarities should be considered:

- In contrast to countries like the UK or the USA, which mainly employ qualified nurses in general practice care, the situation in Germany is different (Goetz et al. 2013). General practitioners' surgeries are an integral part of the German health care system. The individual physician's practice is usually an economic enterprise, which is led by a self-employed physician, as a rule the legal owner and sole holder. In total, there are some 54,000 general practitioners' surgeries. The average general practitioner's surgery has 3.5 fulltime jobs according to infas (2016). In addition to the physicians, the so-called medical assistants (MFA) work in the physician's office. In total in Germany there are 417,000 MFAs. Of these, an estimated 194,000 work in general practitioners' surgeries. These GP practices have similar working conditions, situations and employment relationships. Other working environments of MFAs such as community, specialist medical practices, medical care centres (similar to the UK NHS) and hospitals are not focused in the theoretical considerations.

- From a functional point of view, German general practitioners' surgeries are according to the current EU classification micro-enterprises, since they have fewer than 10 employees. They do not have their own HR department or an organizational structure.

- Measures of primary prevention and general health management measures should be understood as "two-dimensional concept comprising both personal well-being and work-well balance" (Galabova and McKie, 2013). They affect both the private sphere and the professional environment.

If one keeps these circumstances in mind, a universalist and or contingency approach are ruled out. They do not consider the size of an organization as one of the key factors. The universalist approach (Boxall and Macky, 2009) excludes this almost by definition, since it assumes that the success of HRM strategies results from a more or less "right" application of a specific HR practice. The approach to HPWS practices in SMEs is made more difficult by the fact that the starting point for the development of HPWS was the analysis of Japanese high-quality production systems in the 1970s and 1980s as pointed out by Bartram (2005). There is hardly any room in the HPWS 
for the informal practices, which are often a competitive advantage of SMEs (Barney, 1991). Following Kinnie, Hutchinson et al. (2005) the difficulties for SMEs in the contingency approach with the "best fit" approach lie in the assumption that there is a business strategy from which a contingent HR policy can be derived. For example, Bartram (2005) points out that SMEs are unlikely to develop an organizational strategy.

A possibly promising approach for SMEs is provided by resource-based theories. As resources, whatever you mean by them, may well depend on the size of your organization, the theories that are most appropriate for them seem most appropriate for micro-enterprises. According to Marin-Garcia and Tomas (2016) in HRM literature the AMO model is widely accepted to explain the linkage between human resources practices and performance. Following Becker (1992); Appelbaum, Bailey et al. (2000), Boxall and Purcell (2003) the AMO model is an integrative approach, considering both resource-related perspectives, and complemented by sociopsychological components. The AMO model can be seen as a further development of the Rhodes and Steers model (1978) discussed in the previous section. At first glance, the AMO model looks promising. But a more detailed literature review, such as those done by Marin-Garcia and Tomas (2016) in their study "Deconstructing AMO framework: A systemic review", reveals other factors must be taken into account for a more comprehensive approach."

An overview of other factors that go beyond the AMO model as a resource-based approach and influence the HRM performance relationship can be found in Peccei et al. (2013, p. 19). Of particular interest for this research are the paths between HRM practices resp. systems and organizational performance with resources "Employee well-being" and "Employee relationships and coordination".

\subsubsection{Psychological contract and the employee relationship in SMEs}

The fact that the success or the lack of success of HR practices for health management does not depend so much on the way in which they are structured in terms of content brings social and organizational psychological considerations into the discussion. The main question is what influences the HR attributions and thus the employee relationship. The bow is stretched wide. It ranges from the level of trust between employer and employee, historical aspects, management intentions and motivations to legal regulations and trade union organization. "The core point is that, once again, it may not ultimately be possible to gain a proper understanding of the effect of HRM on employee experiences, attitudes and well-being in isolation from the wider organizational, institutional and historical context within which HR systems are embedded and operate." (Peccei et al., 2013, p.28)

The literature search to get an impression of the employee relationship in general practitioners' surgeries and their context is quite unproductive. The topic of human resource management (HRM) in general practitioners' surgeries is unexplored, if you 
expand the topic to HRM in micro-enterprises, you will come across with Mattare, Shah et al. (2013, p. 22) to the description also applicable to general practitioners' surgeries "Since microenterprises - with five or less employees - are the smallest unit possible for a small business, the HR blueprints of these companies are hardly developed and there are no professional HR managers on payroll. At the microenterprise unit level of business, the owner wears the HR manager hat, along with many other hats." At the same time, the outsourcing of HR tasks is not worth mentioning $(<1 \%$ at $\mathrm{n}=959$ ). HR practices in small companies lack information. Two assumptions are typical. On the one hand, it is believed that small business HRM is simply an operational "downsizing" of HR practices in larger companies (Gilbert \& Jones, 2000; Katz, Aldrich, Welbourne, \& Williams, 2000; Kerr \& McDougall, 1999 - cited in Mattare et al., 2013), on the other hand, it is believed that small companies themselves practice a homogeneous system of HRM. In fact, there is as much diversity as there is in larger companies (Mattare et al., 2013).

As Wilkinson (1999) notes, literature on employment relations in SMEs tends to present two ideal types of small business: the "small is beautiful" scenario and the "bleak house" scenario.

- The "small is beautiful" view assumes that there are largely harmonious working relationships. At least insofar as conflicts are not openly resolved. Work is done in a "family atmosphere" characterized by good communication, low bureaucracy, high flexibility and low potential for conflict. The owner is seen as a devoted family head.

- The "bleak house" view (Sisson, 1993) draws the picture of a rather highly authoritarian head of the family, who does not tolerate any contradiction and thus does not cause conflicts. The employees are apparently satisfied, because nobody complains or dares to complain. High flexibility is perceived as a missing system of regulations and orderly procedure. It is seen as the cause of instability.

Between these polarizing perspectives, which certainly also occur in German general practitioners' surgeries, there are many different shades of different forms of human resource management. It is particularly true of medical practices that, according to Wilkinson (1999), is attributed to most employers: "Thus order is seen as the norm and management prerogative is seen as right and not to be challenged: what is good for business is good for employees. Given that many owners define the small firm as their own creation, it is perhaps not surprising that employees should be seen as subordinate to the owner's view of what is best for the firm." (Scott, 1989). Informality and the lack of control systems are justified by the fact that especially small companies enable open communication and the "family atmosphere" promotes building of trust.

This makes it clear that the introduction of health management is not so much a question of appropriate management practices but relies on mutual and reciprocal trust. The emergence and maintenance of trust, in turn, is the subject of the psychological 
contract, which may lead to a better theoretical understanding of the link between HR practices and organizational performance.

Before the psychological contract and in particular its significance for SMEs is discussed in more detail, it should be pointed out that in its counterpart, the legally established employment contract for micro-enterprises such as medical practice in Germany special rules apply. These give the psychological contract a particularly high importance. These rules include employee representation, dismissal protection and occupational health and safety obligations. These regulations place the MFAs in a weaker position in the protection of their interests compared to employees in larger companies.

In "An exploration of small firm psychological contracts", (Atkinson and Cuthbert, 2006) state that a "single typology of small firm relationships" is not possible. From the author's point of view, this is all the truer for micro-enterprises that are even more heterogeneous than the three small firms investigated by Atkinson, with an average of 77 employees per company. The model of a psychological contract she developed can be adopted as a framework through which to reflect nuanced understandings of the employment relationship. In Atkinson (2005) she argues that current approaches are over-generalized and dominated by size determinism. Assuming, like Marlow (2002), that informality is an enduring feature of SMEs, it is clear that an equally informal "mechanism" (Atkinson, 2005) as the psychological contract is promising for the explanation of employment relationship.

The concern of the psychological contract goes back to Rousseau (1989, p. 123): „The term psychological contract refers to an individual's beliefs regard the terms and conditions of a reciprocal exchange agreement between that focal person and another party. Key issues here include the belief that a promise has been made and a consideration offered in exchange for it, binding the parties to some set of reciprocal obligations." Specifically, in the context of the employment exchange relationship employees keep their promises if employers also keep their promises according to Coyle-Shapiro (2002), Coyle-Shapiro and Kessler (2003). "Reciprocity" constitutes a core component in the psychological contract, meaning that employees positively respond to favourable behaviour from their employers (Rousseau \& McLean Parks, 1993). In cases where employees believe that employers have broken their given promises, this will have a negative effect on employee promises fulfilment CoyleShapiro and Kessler (2000).

Characteristic of the approach is, according to the author, that it focuses on the dynamics of the employment relationship. In the course of this, the dynamics of reciprocity are not primarily influenced by the actual, factual fulfilment, but by what is perceived. Following Whitener (1997) in the psychological contract there is a gradual development of the employment relationship, which, in the opinion of the author in a microenterprise, is a permanent change process due to the proximity of the owner and the employee. With each fulfilment or non-fulfilment of the obligations and 
expectations, the complex and finely knit network of relationships changes and raises or lowers the level of trust. Due to the mutual conditionality creates a dynamic, which is characterized by feedback loops.

In the case of an actual or even supposed breach of the psychological contract, employees may experience behaviour such as withholding information, more mistakes, leaving the organization or frustration, fear and mistrust. (Torrington, 2017, p.331).

Referring to the psychological contract for attendance or absence, structural equality is a common feature of the Rhodes and Steers model (1978). The "cyclic nature" of the model is reflected in the escalation cycle of the psychological contract, as the following example shows: The expectation of the two MFAs to the owner physician is the fair treatment (obligation) of both. Suppose that an MFA is frustrated by an unjustified representation scheme, especially since, in her opinion, this has happened several times before. She takes the next opportunity to miss caused by "illness" for two days. Since this seems pretextual, but formally cannot be objected, the employer considers the promise "be honest" (Obligation) as not fulfilled. The absences lead to significant disruptions in practice, which in turn lead to new misunderstandings (unfulfilled expectations) and further disturbances by blaming all parties involved.

Under the aspect of absenteeism reduction, a comparison of the Rhodes and Steers model (1978) with the psychological contract reveals that the latter avoids a weakness of the Rhodes and Steers model. This runs the risk of seeing absenteeism primarily in the individual responsibility and decision-making power and thereby personalizing it. This is evident, among other things, from the fact that the "Personal Characteristics (of the employee)" in the model (cf. Figure 4), is the only element that is not influenced by others. By considering of the (equivalent) "Personal Characteristics of the employer", it becomes clear that he/she, too, may be responsible for the job situation, which then leads to possible absences.

The partners in the psychological contract are the general practitioner on one side and the MFAs on the other. The actions of the physician as agent of the psychological contract largely correspond to one of the management styles exploitative or benevolent authoritative resp. consultative or participative system (Likert, 1967). As owner manager your own autonomy and independence are of importance to the general practitioner. Influenced by the doctor's actions are satisfaction, commitment and motivation.

In the literature on the psychological contract, a distinction is made between transactional and relational contracts. Transactional contracts involve short-term, specific, and monetary in nature beliefs such as competitive wage rates and performancebased pay. Relational contracts involve long-term, less specific, and monetary and non-monetary in nature beliefs such as job security, loyalty, training and development, career development, commitment and trust (Cavanaugh \& Noe, 1999; Robinson, Kraatz \& Rousseau, 1994; Rousseau, 1990 - cited in Katou, 2013, p. 572). 
The Model presented by Atkinson (2005) suggests that transactional obligations within the content of the psychological contract will be predicated upon cognitive trust and distributive justice, whereas relational obligations will be predicated upon affective trust and interactional justice. Cognitive trust can be seen as a trust that is purely rational and calculated, while the affective trust has social relationship and mutual concern (Atkinson, 2005, p. 44). Distributive justice (Deutsch, 1985) is the perceived fairness of outcomes employees receive, drawing on equity theory (Adams, 1965) as a dominant framework; and interactional justice (Bies and Moag, 1986) is the quality of interpersonal treatment received at the hands of the decisionmakers (cited in Atkinson, 2005, p. 39).

According to this categorization, measures of health management in SMEs belong to the relational obligations associated with affective trust and interactional justice. Based on the fact sheets of the CIPD recommendation (2008), these are employerside expectations associated with promises and outlooks of "A pleasant and safe working environment" and "An attractive benefits package". On the employee side these are "Develop new skills and update old ones" and possibly "Maintain high levels of attendance", whereby the latter also partly includes aspects of the cognitive trust.

The above statements show that the question of the effectiveness of health management measures focuses on the mutual fulfilment of the psychological contract. Slightly generalized, according to the author, the psychological contract could be the missing link between HRM and performance.

\subsubsection{The link between HR practices, psychological contract and performance}

Focussing on the fulfilment of the psychological contract at the centre of the analysis of the employment relationship is the central idea of Katou (2013). Her framework, as it will be presented, sees the author as fundamental to HR management measures in SMEs.

Compared to the initially mentioned AMO model, the author believes that this framework has not only a theoretical foundation, but also a statistical validation. The purpose of the study at that time, on which the framework is based, was to examine the extent to which the relationship between HRM and organizational performance changes as a result of an economic crisis, here the financial and economic crisis in Greece (Katou, 2013, p.570). For this purpose, a model is proposed that combines two systems: the psychological contract system and the HR practices system. Figure 7, which summarizes two mappings of the original contribution (Katou, 2013, Figure 1, p.572 and Figure 2, p. 583), shows the overall context.

Before explaining the framework in more detail, it is important to draw attention to a context that is especially relevant to small businesses. Unlike, for example, Tzafrir (2005, p. 1616), which is commonly believed, organisational performance does not 
derive better performance, but rather behaves the other way around. ",.. the negative factor loadings of size item reveal that organizational performance does not follow the economies of scale rule, ..." " (Katou, 2013, p. 584). However, one must put the statement into perspective, since the study was only examined for organizations with more than 20 employees (a total of 177 interviewed organizations with an average of 152 employees and an average of 5 respondents per organization).

In the centre stands, framed by a broken line, the Psychological Contract Fulfilment. Linked to this is the hypothesis that Employee Attitudes mediate the relationship between Employer Promises Fulfilment and Employee Promises Fulfilment. According to Katou (2008), the Employee Attitudes were measured with the characteristics Satisfaction, Commitment and Motivation. As indicators for the Employer Promises Fulfilment ten items of the psychological contract fact sheet of the CIPD (2008) were taken over. McDonald and Makin (2000) distinguish between transactional and relational factors. In the figure, the two transactional factors are highlighted in grey, the remaining eight relational factors have no emphasis. In the same way, the ten indicators for Employee Promises Fulfilment (3 transactional and 7 relational) are presented. As suggested by Coyle-Shapiro and Kessler (2000, p. 912 ff.), not the actual fulfilment but the perceived fulfilment has been questioned. The indicators were ordered according to the confirmatory factor analysis carried out by Katou (2013). The indicator with the higher explanatory value, corresponding to the factor loading, is further to the left. For reasons of clarity, the factor loadings have not been included in the figure. Thus, the indicator for transactional factors with the highest explanatory value for the fulfilment of the Employee Promises is Maintain high levels of attendance (0.57), in the fulfilment of the Employer Promises it is An attractive benefits package (0.59). In the case of the relational factors for the Employee Promises with the highest explanatory value, these are Uphold company reputation (0.59) and Show loyalty to the organization (0.59); at the employer promises they are Interesting tasks (0.61) and Feedback for performance (0.61).

The statistical analysis of the correlations confirms with high (standardized) correlation coefficients the relationship Employer Promises Fulfilment on Employee Attitudes (0.94) as well as the relationship of Employee Attitudes to Employee Promises Fulfilment (0.86). Employee Attitudes indicators are almost equivalently relevant satisfaction (0.67), commitment (0.64), motivation (0.65). This confirms the hypothesis on the functioning of the psychological contract system at a high level of significance ( $\mathrm{p}<0.01$ ). The Employee Attitudes form the heart, as Boxall and Purcell (2003) put it, or the epicentre, as Katou (2013) puts it, of the psychological contract system.

If one as the author follows this hypothesis, it is to be assumed that also in German general practitioners' surgeries the Employee Attitudes strongly influence the fulfilment of the Employee Promises and that they themselves are strongly influenced by the fulfilment of the Employer Promises. Introducing health management, and promoting personnel prevention activities, could create Opportunities for training and development and Opportunities for promotion on the part of the employer. In addi- 
tion, an A pleasant and safe working environment could be supported. This would not only improve the transactional conditions for Fulfilling Employee Promises, but also the relational factors of Loyalty to the organization, Uphold company reputation, and Develop new skills and update old ones. If the physician, as an employer, also make use of the opportunity to delegate primary prevention tasks to the MFA, he would promote the factor that has the greatest impact on employee Satisfaction, Motivation and Commitment, namely, Interesting tasks.

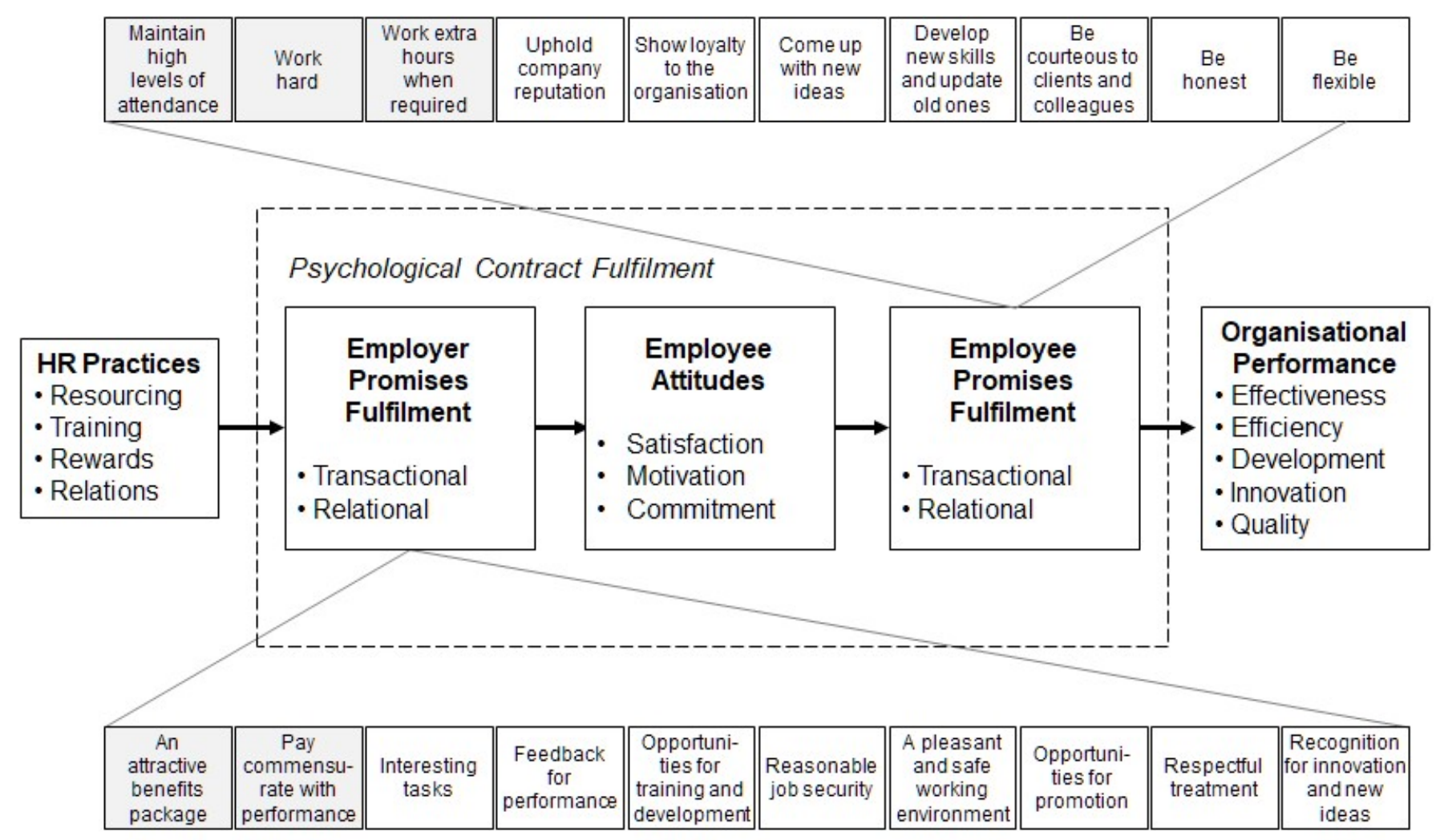

Figure 7: An HR practices - psychological contract - organizational linkage framework (Katou, 2013, p. 572 combined with p. 583)

From the order of sorting the transactional factors, it becomes apparent that action on the factors Pay commensurate with performance and Maintain high levels of attendance should come first. As Atkinson (2005, p. 51) points out, the transactional factors are to be seen as hygiene factors in sense of Herzberg (1959). This means that first, the consistency of the transactional factors must be present before positive effects can be expected through relational factors. Katou (2013, p. 586) confirms this view by comparing the standardized coefficients, stating, "Therefore, our findings support the view that transactional aspects of the psychological contract must be addressed (Millward and Brewerton, 1999), because transactional aspects drive relational aspects due to their cause and effect association (Pate et al., 2003). " 
The second system in the framework, the HR Practices system, is hypothesized to mediate the relationship between HR Practices and Organizational Performance through the Psychological Contract Fulfilment. This means that the HR Practices via the Employee Attitudes influence the Organizational Performances. This is confirmed by high standardized correlation coefficients between HR Practices and Employee Promises Fulfilment (input) and between Employee Promises Fulfilment (outputs). The hypothesis was reinforced in the study by Katou (2013) by testing the direct connection between HR Practices and Organizational Performance, i.e. without the interposition of the psychological contract, and rejecting it for lack of significance. "This means that better HR practices will produce superior psychological contracts, which by incorporating healthier employee attitudes will improve organizational performance"(Seeck and Parzefall, 2010) (Seeck \& Parzefall, 2010).

The interviewed HR Practices consisted of 12 items that were developed by Armstrong (1996), and grouped into four areas: Resourcing (recruitment, selection, flexible work arrangements), Development (work design, performance appraisal, training and development), Reward (compensation, promotion, incentives) and Relations (participation, involvement, communication). Each of the components of employee Rewards is capable of producing psychological contract by establishing the belief that organization's employees worth their long-term salaries (Gomez-Mejia, 2004). Good employee Relations produce positive feelings to employees who accordingly keep their promises to organizations (Robinson, Kraatz et al., 1994). Among the HR Practices with the highest impact on Employee Promises Fulfilment are promotion (rank 3) and participation (rank 5).

Although HR Practices do not explicitly mention health management and prevention, it can be assumed that they are also considered a Reward (promotion) by the general practitioner and thus strengthens the fulfilment of Employee Promises. If, for example, the development of preference profiles increases the participation rate among the MFAs, the promises of the personal contracts can also be fulfilled more easily.

Organizational Performance is measured by the indicators Effectiveness (0.61), Innovation (0.60), Development (0.58), Quality (0.53) and Efficiency (0.48). In brackets the standardized coefficients. After that, Effectiveness is most strongly influenced by the fulfilment of Employee Promises.

The specific items constituting the Organisational Performance construct include: Effectiveness - if the organisation meets its objectives; Efficiency - if the organisation uses the fewest possible resources to meet its objectives; Development - if the organisation is developing in its capacity to meet future opportunities and challenges; Innovation - for products and processes; and Quality - per cent of products of high quality, adopted from Delaney and Huselid (1996).

The link from Employee Promises to Organizational Performance especially for general practitioner's surgery or health management is only discussed in a few studies. In his "Socio-technical System Analysis and Assessment in Medical Practices" 
by Majumdar (2004), all aspects of performance are addressed holistically, but the Psychological Contract remains outside; at best, the work-organization cooperation with the assistant, the former name for the predecessor profession of MFAs, is discussed. One focus is more on the management skills and especially the selfmanagement of the physician. A distinct connection is made by Szecsenyi et al. (2011), evidencing a clear link with patient satisfaction, a component of Quality management, quasi from the centre of the Psychological Contract, employee Satisfaction. Kathmann and Dingeldey $(2013,2014)$ show that breaches and violations of the Psychological Contract, such as precariousness pay and job insecurity, can lead to performance impairments. Moeller and Babitsch (2017) emphasize the importance of prevention and health promotion for MFAs $(n=43)$ and derive from this a sustainable further education concept for the workplace.

Overall, the theoretical framework proposed by Katou (2013) can contribute to a better understanding of the people and performance link by embedding one system (psychological contract system) into another more comprehensive system (HR practice system). Through the reciprocal fulfilment of promises, but also through the nonfulfilment of promises as well as concrete breaches of contract and unfulfilled expectations, the history of the organization is expressed. This creates, in the author's view, a complex dynamic that is difficult to control, which makes it difficult and perhaps completely impossible to illuminate the initially mentioned "black box" and turn it into a "white box" - maybe the box is more like a kaleidoscope.

For the aspired priority concept with the promotion of personal health potentials of the MFAs in German general practitioners' surgeries the framework offers the possibility to locate health management in a holistic concept for practice management. The health promotion measures themselves constitute an HR Practice that allows the physicians to increase Satisfaction, Commitment and Motivation in fulfilment of their promises from the Psychological Contract. In doing so, employees can increase the Organisational Performance of their general practitioner's surgery, in terms of Efficiency and Quality, in fulfilling their part of the Psychological Contract. The next section is therefore about the implementation.

\subsubsection{About the benefits of health management}

"It [well-being] represents a broad bio-psychosocial construct that includes physical, mental and social health" (CIPD, 2016, p. 4). The initiative to act and promote personal health potentials in general practitioners' surgeries could, as Greasley, Edwards et al. (2012) express, be a "win-win" situation that meets both employers' and employees' organizational interests.

As far as physical health is concerned, as Marsden and Moriconi (2009) conclude "There is now a substantial body of research indicating that the kind of policies used by PFW [a British company] to improve employee health and promote more healthy life-styles are potentially beneficial to organisations. ... Some aspects of people's 
life-styles are particularly associated with higher absence rates, including smoking, heavy drinking, drug abuse, and lack of exercise." In the author's opinion, this statement should also apply to general practitioners' surgeries and is a reason why, in a first step, in health management measures, primary prevention to improve physical health is first recommended. Especially when it comes to physical well-being, the "win-win" situation at the primary prevention measure becomes apparent with influenza vaccination. It has been shown to significantly reduce absence days and leads to the worker avoiding physical discomfort associated with any flu.

The connections become more problematic regarding the mental and social wellbeing. Although the following statement from (Greasley et al., 2012, p. 444) refers to call centres, it can be applied to medical practices where the task can certainly be classified as stressful: Baldry, Taylor et al. (2006, p. 252) conclude from their research on call centres that "when a task which is itself stressful" is performed in an "unsupportive" work environment that requires extensive interaction with technology above recommended levels, "a reduction in employee health and well-being must inevitably occur". This illustrates that the value of the resource human capital lies not only in physical well-being, but also in mental and social well-being and their interplay. Here it becomes apparent that, depending on circumstances, HR practices can also lead to "win-lose" situations. If it becomes obvious to the employee and transparent that, for example, his or her health should only be promoted so that it is even more efficient in the sense of the organization, this will sooner or later have a lesser effect on motivation and decreasing engagement.

A more detailed analysis of the role of employee well-being in the HRMorganizational performance relationship was provided by Peccei et al. (2013). Based on two perspectives identified by Peccei (2004) on the HRM-WB-OP relationship, the so-called "optimistic" and "pessimistic", and a comprehensive literature review by Van De Voorde, Paauwe et al. (2012) they discuss three scenarios of the HRMWB-OP relationship:

- Weak mutual gains: This simple win-win situation scenario results and improvements to well-being as well as organizational performance for an HR action. But these results are not interrelated; they are to be considered in isolation from each other.

- Strong mutual gains - As with the weak mutual gains' scenario, HRM affects both $\mathrm{WB}$ and $\mathrm{OP}$, in addition to a reinforcing effect, in that $\mathrm{WB}$ itself has a positive effect on OP.

- Conflicting outcomes - This is a simple win-lose scenario where HRM proves useful for organizational performance, but at the same time, it is detrimental to the wellbeing of the employee. This model suggests that performance increases are due to exploitative HR practices or that there are conflicting goals. 
Health management measures are likely to lead, at least as a rule, to weak mutual gains. Since they also influence the factor "job satisfaction", they lead to an increase in customer satisfaction, at least in the professional field of MFAs, as shown in Szecsenyi et al. (2011), a double effect can be expected, i.e. strong mutual gains. However, primary prevention measures specifically for MFAs should not be overlooked as leading to more responsibility. As stated in Regus (2012), and Babitsch and Von Moeller (2017), this may lead to a change of tasks. The MFA takes over tasks such as for example vaccinating, with medical content. In discussion is whether this achievement is specially rewarded. In any case, delegating tasks from the physician to the MFA increases their responsibilities, and this, in turn, is often perceived as an increasing pressure to perform and stress, which can lead to conflicting outcomes.

Nishii, Lepak et al. (2008) draw attention to the fact that the success of health and well-being measures depends not so much on their content, but on the managementphilosophy recognized by the employee. In the typology they propose, management philosophies can be attributed to service quality, cost reduction, employee wellbeing, exploiting employees, or union compliance (Nishii, Lepak et al., 2008, p. 509). Depending on the perceived management philosophy, the practices are differentially successful. Quality and well-being attributed ones are more associated with an increase in employee satisfaction and commitment (strong mutual gains), while cost or exploitation attribution tends to reduce employee satisfaction and commitment (conflicting outcomes).

\subsection{Bridging the implementation gap}

While in the previous sections the theoretical conditions under which HR practices could be successfully used to reduce absenteeism and to increase performance are discussed, this section focuses on the transfer of HR practices from theory into practice. Considerations for the fulfilment of the psychological contract as well as the development of the priority concept and its implementation are contributions to closing the gap. The basis for this is a participative decision-making concept and a standardization proposal for health management in SMEs developed by Walter (2007). Finally, it is explained and clarified why in German general practitioners' surgeries the HR practice of primary prevention should be implemented first, ahead of other conceivable activities such as reorganization of work or change of job design.

The framework presented in the previous section (Katou, 2013) helps to improve the theoretical understanding of the link between HR practices and possible performance enhancements. The focus is on content considerations. For application oriented HRM research, however, questions of implementation, that means questions about the process of introduction, are becoming increasingly important. Torrington et al. (2017, p.80) note that intended strategies, policies and HR practices are not implemented as planned, which may lead to disruption and impairment of the targeted performance improvements. Biggs, Abel et al. (2011, p.169) refer to Knight, Cowling et al. (2006) 
and state: "The implementation crisis in conservation planning is one of conservation's greatest challenges. Although conservation planning is a social process that encompasses assessment, planning, and sustained management of areas important for achieving conservation goals, conservation planning approaches continue to emphasize ecological over social considerations." Wang, Zhu et al. (2009, p. 63) consider the "implementation gap" in addition to "apperception gap" and the "system gap" to be one of the key influencing factors in the formation process of employee satisfaction. The implementation gap itself, they see as "caused by the system constituted has not been truly implemented." These and other gaps are closely related to the psychological contract.

In this section, therefore, the psychological contract and the decision-making once again are broached, as they seem to be central both for the HR process itself and for the implementation process. Behind every implementation is a decision-making process. If the employee is involved in this process and realizes why the decisions fall as they are, the implementation is smoother.

\subsubsection{The implementation process and the psychological contract}

The HR-PC-OP framework presented in the previous section (Katou, 2013) provides a first important implementation suggestion with Atkinson's (2005) note on hygiene factors. First, the mutual promises on the transactional factors of the psychological contract must be fulfilled before performance increases can be achieved through increased satisfaction, motivation, and commitment. Health management relies more on the relational components of the psychological contract. From this point of view, its implementation in a general practitioner's surgery, where the transactional conditions are not correct, where precarious pay and marginal workloads are present, is doomed to failure.

Another aspect that pertains to the fundamentals of the psychological contract should be noted. No matter what is implemented, after implementation, the organization is different than before. If it is not clarified beforehand whether the employee wants to apply the psychological contract to the newly developing organization, in the figurative sense of the contract extended, the desired changes, even if the transactional conditions are right, are also hardly feasible. If the change causes the employee to work for an organization for which he did not originally serve, it is all too quickly perceived as a breach and violation of the psychological contract with all its negative consequences. It is not without reason that Oreg, Michel et al. (2013) point to the critical importance of the workers' perspective. An aspect that should be considered, for example, when - as in Mergenthal, Beyer et al. (2013) explained - MFAs are to be increasingly used in home-centred care (VeraH) due to their improved primary prevention skills, which will then be used for home visits. This argument is in line with Marsden and Moriconi (2009, p. 5), who notes: "For example, an emphasis on improving health may also signal to employees that their employers wish to change the 
psychological contract. Equally, it may be hard for an employer to change the psychological contract without emphasising the value of employee health."

The employee's question about the "why" (Nishii, Lepak et al., 2008) or, more generally, "sense-giving", raises an aspect of the implementation process that, although not a direct part of the psychological contract, has a lasting effect on its fulfilment. It is the question of the credibility of the employer and his/her motivations associated with the introduction. Shipton, Sanders et al. (2015) found that where employees perceived that HR practices were implemented with an eye to their needs and aspirations, they were more inclined to report positive attitudes and to behave in a way conducive to the achievement of organisational goals. Nishii, Lepak et al. (2008) draw attention to the fact that the success of health and well-being measures depends not so much on their content, but on the employer's management-philosophy and the motivation behind recognized by the employee. These questions have a special significance for a physician's practice, as described in more detail in Majumdar (2004), because there is a constant balance between the medical claim of healing and commercial interests.

As with all primary prevention measures, the role model of the physician is also important as it enhances the previously mentioned credibility. The leaders of a general practitioner's surgery need to take personal responsibility and a role model here (Gerlach (2001) cited in Amend (2005, p. 73); Bruch and Kowalevski, 2013). Although the role model function is not a direct matter of the psychological contract, it is obvious, at least in a general practitioner's surgery, that primary prevention can hardly be successfully implemented if the responsible physician itself interferes impair with personal health potential.

The last note addresses another fundamental implementation problem. Legal and psychological contracts essentially relate to the professional environment. Primary prevention, however, affects both the private and the professional environment. CSR is increasingly recognizing and accepting that companies are responsible to society and employees for "healthy" jobs. But there remains the question of whether it can derive from the claim to interfere in the private life of the employee, or whether this recognized as a breach and violation of the psychological contract. Primary prevention measures are predominantly life-style measures and can be regarded as an intervention in the work-life balance. In order to avoid misunderstandings on this point, measures for employee participation and involvement should be taken at an early stage for the implementation in order to identify possible potential for conflict as well as preferences and to include these in the decision.

The last point outlines the theme of the second research question.

1. Which of these measures are already practised and which are preferred by the staff of the general practitioners' surgeries? 
The result is a preference profile with the proposed measure based on the evaluation of survey results, the MFA survey.

\subsubsection{Decision-making for implementation}

"To them [the employees] this means that, "more effort should be devolved to finding out what managers are thinking and why they make the decisions they do." (Becker and Gerhart, 1996, p. 794 - cited in Kinnie et al., 2005, p. 10). This quote makes a good transition to the actual decision-making process. It is assumed that the more transparent the decision criteria, the weighting, relevance and evaluation, the more acceptable a decision becomes. The aim is to develop a concept of priorities that, in the sense of the psychological contract, can be understood by both the employer and the employee and, if necessary, adapted to the specific situation. The related research questions are:

2. On what basis can the implementation of these activities be prioritized by a multiple criteria decision-making model, which considers relevant stakeholder perspectives?

3. How can the procedure of decision-making be adapted to the specific conditions of an individual general practitioners' surgery?

The decision-making process itself is a rather methodical question and is therefore dealt with in more detail in the chapter "Methods". From the framework, the psychological contract as a stakeholder perspective yields both the employee view and the employer view. The employee view flows in via the results of the preference profile as a decision criterion for acceptance, since a successful implementation without acceptance can hardly succeed. The employer view is represented by the decision criterion costs. Organizational performance as part of the framework results in the third decision criterion, effectiveness.

The result of the multi-criteria decision-making model is a list of priorities with suggested primary prevention measures proposed to the general practitioner's surgery for implementation. This creates the basis for an efficient implementation that can take advantage of standardization.

Since "one size does not fit all" (Kinnie et al., 2005), starting points for adaptations are shown that may change the sequence shown in the priority concept.

\subsubsection{Approaches to implementation}

The introduction of activities promoting personal health potentials can take different forms. The chapter "Discussions" investigates three approaches that build on each other. The first sees the promotion of personal health potential as a more or less unique, situational action. The setting is: The general practitioner is convinced of the correctness of the concept and now plans to implement it as quickly as possible in his 
own practice without any further implications. The second setting sees the standard proposal on primary prevention as a first key step towards a more comprehensive and ambitious doctor's health management project, based on the recognition that employee health can be a key success factor for the practice and, in the longer term, not to be underestimated represents a competitive factor. From a holistic view of practice management, health management can be embedded in a more comprehensive quality management system. These approaches are the subject of the research question:

5. What approaches should the general practitioner (as owner) consider if he/she wishes to set priorities for the introduction of a health management system?

In order to reflect the outcome of the MFA survey and the established priority concept, structured qualitative interviews were conducted with selected general practitioners.

The necessity and the benefits of introducing a health management system have been proven in several studies. There is a regular report from the Government on the German health management. The IGA report by Sockoll, Kramer et al. (2006) provides a good overview of the health management activities in general. In the "Health Management in Small and Medium-Sized Enterprises - Results of a Representative Survey" Zelfel, Alles et al. (2011) showed that one third of the German SMEs do not have any health management and that there is a need to improve the health management in German SMEs and that the health management is not established in German SMEs. The smaller the enterprises the less health and well-being programs are found. Small and medium-sized enterprises have a need for consultation in cases of illness or health prevention. However, there is not yet an organised structure available for getting advice.

Implementation means integrating a subsystem into a more comprehensive system. However, standards based on experience and proven concepts are helpful to bridge the implementation gap especially with SMEs, who often cannot afford to learn the hard way. This is presented here for the field of health management.

Standardization can contribute to closing the implementation gap. This applies in particular to measures of quality management. "One of the mainstays of TQM is standardization in Japanese understanding. There must be clear standards for every work process - as a necessary tool for permanent review and improvement of quality. At the same time, standards are not formulated for every single work step, but merely standardized and therefore measurable. The task of the management is to set the standards and to ensure that all employees work according to these standards. It is also the task of the management to create the conditions for constantly questioning existing standards and replacing them with new, better standards. "Imai (2002, p. 126f.) cited in Walter (2007) - translated by the author. 
Especially in the medical field, standardization has become established in the form of guidelines. According to Timmermans and Berg (2003) they serve as the basis for good results for the contents of medical work and the concrete actions of physicians. Standardization supports a systematic approach and provides a benchmark for assessing progress in the introduction, assessment, establishment and stabilization of management activities. Under stakeholder aspects, standardization helps to improve the transparency and traceability of decisions and coordination measures. By allowing standards to be applied independently of organization-specific issues and goals, they offer time and cost advantages, helping to reduce the implementation gap.

For the standardization of health management, Walter (2007) has developed a target system for SMEs, which can also be used as a guideline for general practitioners' surgeries. As demonstrated in the next section, it also allows the prioritization of health management measures.

Following Walter (2007, p. 217) four major goal areas can be identified for the standardization and implementation in small enterprises in the sense of a balanced scorecard (Kaplan and Norton, 1996). Each major goal is divided into three subgoals:

- $\quad$ Development and permanent anchoring of the management system (1)

- Development and optimization of employment policy requirements

- Design and optimization of structural and planning framework

- Execution and optimization of core processes

- $\quad$ Strengthening the social and human capital (2)

- Health promoting work organization (2.1)

- Health promoting organizational design (2.2)

- Promotion of personal health potentials (2.3)

- $\quad$ Improvement of well-being and health (3)

- Increasing emotional well-being (3.1)

- Improvement of physical health (3.2)

- Reduction of risk factors (3.3)

- Improvement of productivity, quality and economy (4)

- Improvement of performance and productivity (4.1)

- Improvement of quality and customer focus (4.2)

- Cost improvement (4.3)

These goals are used in the following subsection to discuss the target system and its interdependencies. In doing so, the respective sub-goals were taken over for prioritization - except for the first goal, the development and permanent anchoring of the management system (1). Because the management system consists of the general practitioner, the main success factor is the leadership ability of the physician. She/he defines the company policy, manages the planning framework and monitors the es- 
tablished processes in the framework of Quality Management Guidelines (QM-RL) for SHI-accredited physician (G-BA, 2016).

This research contributes to the standardization of measures to achieve the sub-goal 2.3 "Promotion of personal health potentials (primary prevention)". The aim of this work is, among other things, to provide decision-makers with a standardized and well-grounded concept for achieving goals in such a way that the stakeholder perspectives integrate employees, effectiveness and costs.

An important issue for the implementation is the finding that the goals and objectives are not independent of each other. They can complement each other or conflict according to Zangemeister (2014). For a promising implementation, it is therefore important to identify those goals that have the highest possible reinforcement effect. For example, improving physical health (sub-goal 3.2) is usually associated with an increase in emotional well-being (sub-goal 3.1) and a reduction in health risk factors (sub-goal 3.3) and vice versa.

\subsubsection{Deriving priorities for German general practitioners' surgeries}

The previous sections are focused on "Promotion of personal health potentials". But this is just one possible starting point and one sub-goal to establish a comprehensive health management system. This subsection is about depicting the previously described dependencies in the target system and then providing a systematically derived and theoretically sound recommendation for the implementation priorities. At the same time, it justifies why the priority concept presented in this research focuses on primary prevention as a first implementation step and not on other areas of a health management system such as work organization or job design.

Methodically the derivation of priorities is based on a cross-impact matrix in which the reciprocal influence of the goals is evaluated as proposed by (Vester, 2009). As suggested by (Gomez and Probst, 1997), the dependencies are used to find out the changes with the largest control lever; the so-called "steering abilities". Following a suggestion by (Wilms, 2012), a priority concept for the implementation can be created.

The contexts developed in this subsection are taken up in the chapter "Discussion" and help to answer the research question:

5. What approaches should the general practitioner (as owner of the practice) consider if he/she wish to set priorities for the introduction of a health management system?

In addition, this subsection provides concepts on which the qualified interviews with physicians will rely on.

Content of the derivation of priorities is based on Walter's goal system (2007) and supplemented with aspects of the causal model of absenteeism (Brooke, 1986), the 
revised Rhodes and Steers model (1978) presented at the beginning of this subsection. To illustrate the relationship to Walter's goal system (2007), the goals and subgoals are highlighted in italics.

\subsubsection{Leadership ability}

If one follows Walter (2007), the development and permanent anchoring of the management system is of central importance for the establishment health management in SMEs. Only if the topic of health management is permanently present and incorporated in all important planning and organizational decisions can health management succeed. Transferred to the general practitioner's surgery, this means a high standard of the general practitioner's leadership ability. Regardless of the management style, it is by no means self-evident that in the general practitioner's surgery, beyond the legal requirements, the creation of a health-promoting environment is more successful than in other sectors. Skills relevant to leadership, motivation, work organization and health management are usually not taught in the education and training of primary care physicians. In the following it is shown to what extent the literature review can be used to derive relationships between the leadership ability and other goals.

The direct influence of the owner's leadership abilities on an improvement of productivity, quality and economy is well documented. Frodl (2016) sees the core tasks of practice management as reducing costs and improving efficiency. The relationship between leadership ability and cost improvement is presented in Köper, Möller et al. (2009) and Amend (2005). The improvement of quality and customers focus depends mainly on the ability of the physician to lead the patient. He/she is obliged, as pointed out by Köper, Möller et al. (2009), Marcks (2012) and Amend (2005), to ensure the quality standard and the customer orientation of the surgery. The exemplary role of "healthy" leadership demonstrated by Bruch and Kowalevski (2013) has an impact on personal health potentials (prevention). Thus, the physician does not appear particularly credible if he / she maintains a personal lifestyle that cannot just be described as beneficial to health - especially since this will not be hidden because of the proximity of the MFA.

The factors 'organisational permissiveness' and 'kinship responsibility' identified in Brooke's (1986) causal model of absenteeism (see section 3.1.2) are directly related to the relationship leadership ability and improvement of productivity, quality and economy. It corresponds to the idea of an "absence culture" (Johns \& Nicholson, 1982; Nicholson, 1977 - cited in Brooke, 1986, p. 353), and can be considered empirically proven for the 'organisational permissiveness' (Seatter, 1961, Rhodes and Steers, 1981; Winkler 1980, Popp and Belohlav, 1982 - cited in Brooke, 1986, p. 353). Regarding 'kinship responsibility', especially for MFAs, it is important to note that this leadership aspect is of importance, as part-time work is very important, as described in subsection 3.1.1, and women's income is essentially ancillary, subordinated to family interests if necessary. The factor 'distributive justice' in Brooke's (1986) model can also be attributed to leadership ability. However, it does not direct- 
ly affect productivity, quality or economy, but indirectly impacts emotional wellbeing, job design and work organization.

Evaluating the implications of leadership ability under the aspect of a psychological contract (Atkinson, 2008; Katou, 2013) and its fulfilment, as discussed in section 2, the transactional promises refer directly and clearly to productivity and economic goals. Quality and customer focus are paramount in relational promises such as 'reputation, loyalty and courteous'. Employees' expectations are largely based on emotional well-being and work organization (e.g., 'pleasant and safe working environment').

\subsubsection{Strengthening the social capital and human capital}

In addition to the leadership quality of the physician, the content of the vocational training of the medical assistant (MFA) are crucial for a health enhancing job design. The occupational profile is very wide and holistically and thus meets the requirements of the principle of harmony: task - request - responsibility must stand in line. Following the BIBB survey (Bundesinstitut für Berufsbildung BIBB, 2012) (Federal Institute for Vocational Education and Training), the general job design of the MFAs seems to be alright: $87 \%$ of the MFAs confirm in the survey that working is adequate to their vocational training; compared to $77 \%$ for all the professions (Figure 8).

Improvements for a health enhancing job design directly affect the organisation of work. The link to the organisation of work is addressed at Berger et al. (2008), Larsson, Landstad et al. (2009), and Amend (2005). An example of how job design can affect the work organization is participation. "Participation is another success factor dealing with work health promotion and organizational development." (Juran, 1989, cited in Larsson et al., 2009, p. 55). Employees who are involved in the design of work organization, better control generally the work processes and respond in flexible, if there are deviations from the standard. An improved health enhancing work organisation in turn promotes the emotional well-being (Graham et al., 2010; Larsson et al., 2009), as stress-producing and conflict-inducing procedures are avoided.

There are many direct references from a health enhancing job design to the emotional well-being. Work overload and mental underload, unsatisfactory task allocation, low self-realization, pay and work schedules are referred as tags to indicate the link (Graham et al., 2010; Saari, 1981; Berger et al., 2008; Hausknecht et al., 2008; Larsson et al., 2009). This link seems to be the reason for Hausknecht's statement: "The lowest amount of absenteeism which found among work units where job satisfaction and organizational commitment were both high." (Hausknecht et al. 2008, p. 13)

A health enhancing work organization directly affects emotional well-being (Graham et al., 2010; Köper et al., 2009; Larsson et al., 2009). 82\% of the physicians questioned by Amend (2005, p. 96) see in the practice organization a significant im- 
provement potential. $78 \%$ see in the foundations of the work organization and time management improvement areas in the work spectrum of the employees.

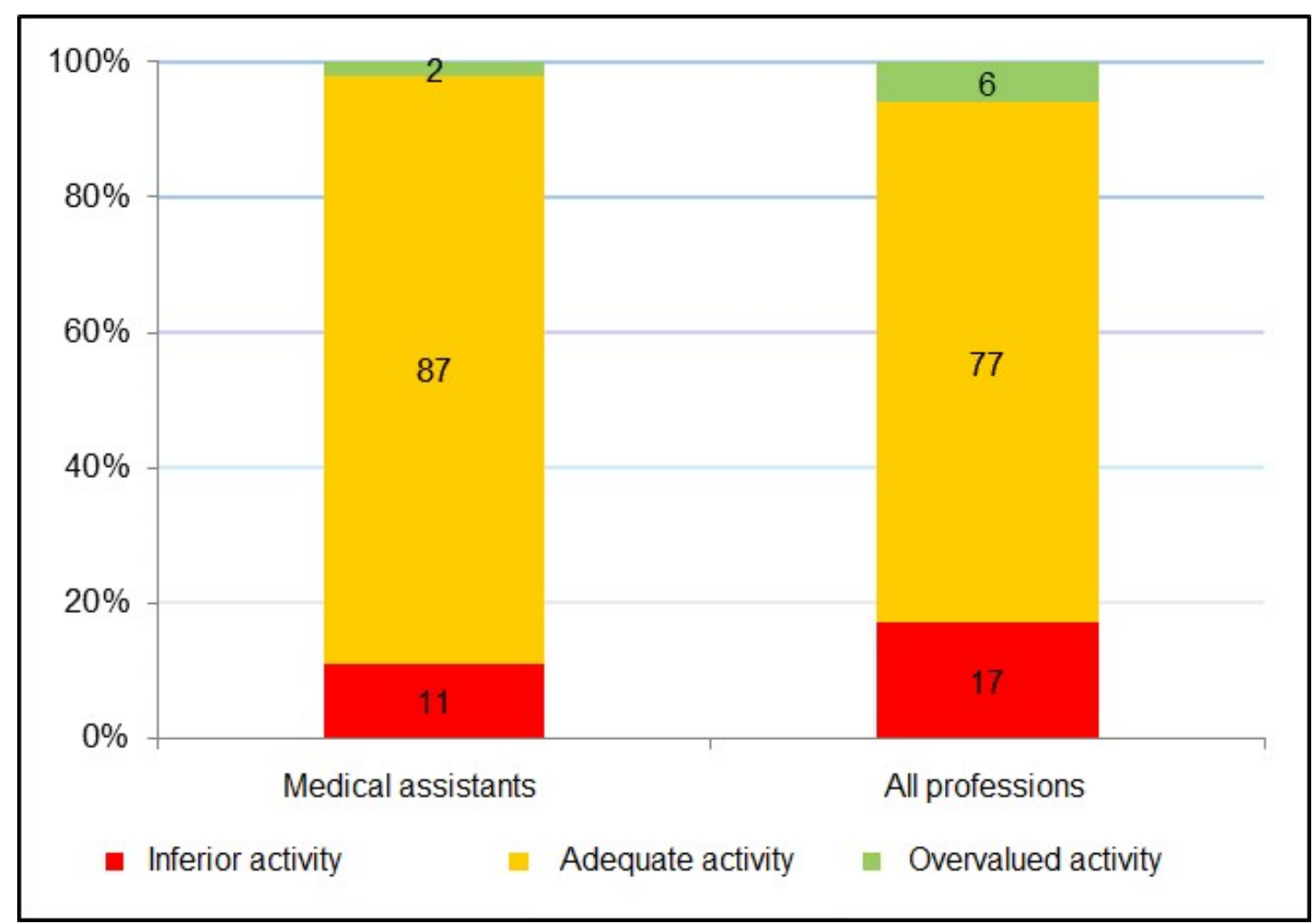

Figure 8: The job design of MFAs fits to the vocational training (source: Bundesinstitut für Berufsbildung BIBB, 2012 - translated by the author)

In subsection 2.1.4, the main measures for the promotion of personal potentials (prevention) have already been presented: Nutritional behaviour, physical movement, coping with stress, drug prevention and vaccination to strengthen and build up the defence and immune system. They affect the physical as well as the emotional wellbeing as stated by Handschuch, Schreiner-Kürten et al. (2015). Prevention measures that influence physical health are described in detail in Brewer et al. (2006); Laaksonen et al. (2009); Little et al. (2015); Vagholkar et al. (2008), Köper et al. (2009), Bräunig (2015), and Saari (1981) demonstrate the influence on emotional well-being.

All the relationships shown so far, apart from an exception, are directly evident from the literature search. No robust studies could be found for the relation between personal health potentials (prevention) and quality and customer focus. However, this is due to the special characteristics of a general practitioner' surgery as described in subsection 2.2.7 (Diseases and disease prevention at the workplace). The physicians estimate their own consulting expertise not only for the prevention measures (Figure 3) but are also ready and willing to delegate measures to the MFAs. At least in consultation with nutritional behaviour, physical movement, about $80 \%$ of the physicians consider a delegation to the MFAs (Figure 9) to be "clear" or "rather" meaningful. Concerning smoking, only a small majority considers the delegation to be mean- 
ingful. "In the case of alcohol consumption, stress and sexuality, on the other hand, this is less sensible at all. On the topic of sexuality, significantly more male physicians advocate the delegation of counselling as female physicians". (Regus, 2012 translated by the author). In the field of vaccination, the Prevention Act created the prerequisites for the MFAs to be able to perform this physical (and thus operational) intervention.

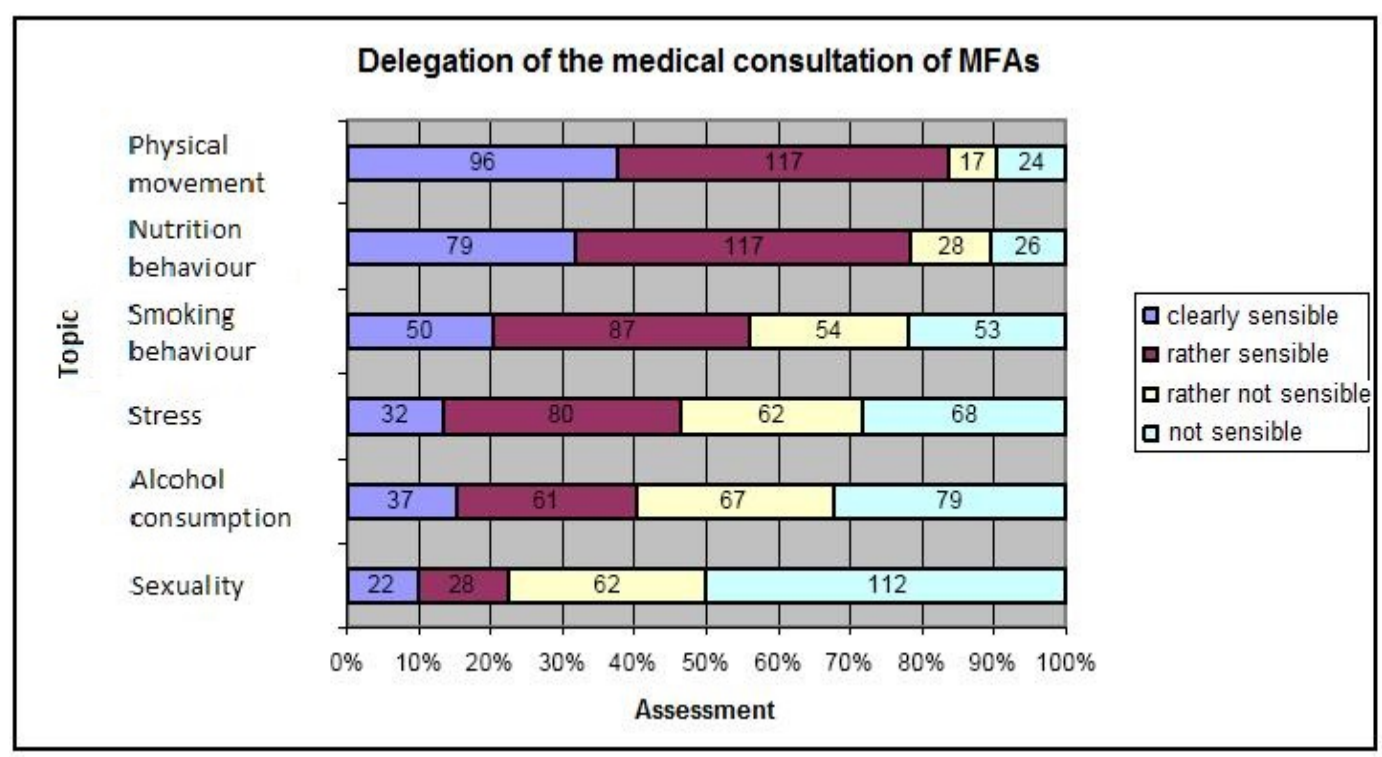

Figure 9: Assessment of the delegation of preventive advice to MFAs with absolute and relative frequencies; According to the frequency of the assessments "clearly sensible" and "rather sensible" - (Regus, 2012)

By taking on advisory tasks for prevention, the MFA contributes to the quality and customer orientation of the general practitioner's surgery. From this point of view, the impact of personal health potentials (prevention) to the quality and customer focus must be weighted high. An MFA, who herself makes preventive measures, makes this with a higher competence and thus she increases the quality of the surgery.

In Brooke's (1986) causal model of absenteeism (see section 3.1.2), the factors 'work involvement' and 'role ambiguity, conflicts and overload' belong to the target area of strengthening the social and human capital. 'Work involvement', defined as a normative belief in the centrality of the work role in one 's life (Kanungo, 1982 - cited in Brooke, 1986), not only has a direct effect on reducing one's absence, but also indirectly in multiple ways. On the one hand, it increases the involvement of the job, which in turn leads directly to the reduction of absence and, indirectly, also leads to a reduction of the commitment by reducing the commitment. (Goodale, 1973, Ilgen \& Hollenback, 1977, Rabinowitz \& Hall, 1977, Kanungo, 1982). Expressed in the categories of Walter (2007), there is a strong influence of 'work involvement' on job design and work organization, which continues via emotional well-being. 
The role-based factors have a direct impact on work organization and job design in Brooke's (1986) improved Rhodes and Steers (1978) model. Moreover, they directly affect the emotional well-being as well as the physical health. A special emphasis is placed on the factor 'alcohol involvement'. It leads in the target model of Walter (2007) to an increase in the risk factors. "Despite these empirical links between highlevel alcohol involvement and absenteeism, and the common wisdom of most experienced supervisors and managers, organizational scholars have almost totally ignored the potentially important explanatory power of this variable." (Brooke, 1986, p. 355).

As stated in Atkinson (2005), the employer is the "contract holder". This property means the power of disposition over social and human capital., a key area of health management according to Walter (2007). The "currency" of the contract, according to Atkinson (2005), is trust, which, according to Katou (2013), in the psychological contract results in an increase in satisfaction, motivation and commitment and the associated emotional well-being.

\subsubsection{Improvement of well-being and health}

This goal includes physical health and social and psychological well-being (emotional well-being) as well as potential risk factors. First of all, the dependencies of these sub-goals should be analysed.

A first step will focus on the causes of health risks. Here, roughly speaking, physical health and emotional well-being must be distinguished. Both affect the health risk factors. In their studies Abraham and Graham-Rowe (2009); Dugdill, Brettle et al. (2008); Laaksonen, Piha et al. (2009); Van Strien and Koenders (2010); Köper et al. (2009); and Vu-Eickmann et al. (2017), show how an improvement in physical health leads to a reduction in risk factors. Saari (1981); Hausknecht et al. (2008); and Vu-Eickmann et al. (2017), confirm that the improvement/deterioration of emotional well-being also leads to the reduction/increase of risk factors. Both factors themselves influence each other. Physical health promotes emotional well-being. The resulting cycle is escalating - physical pain quickly leads to a bad mood. A bad mood affects the social contacts, which then do not pity, which in turn increases the pain ("shared suffering is half the sorrow"). This and similar contexts are explained in Saari (1981) and Vu-Eickmann et al. (2017), and by Majumdar (2004, p. 114) it is integrated into the socio-technical system of physician's surgery: "to look at the atmosphere" is regarded by many doctors as basic management behaviour. A second important cycle is addressed: The interaction between leadership ability and emotional well-being of practitioners. The challenge is to keep stressors and motivators (resources) in balance. Details to the cycle contain Graham et al. (2010); Saari (1981); Köper et al. (2009) and Vu-Eickmann et al. (2017).

In the case of competing goals, the effect has an inverse direction. If there are fewer health risk factors, such as described in subsection 2.2.5, the performance and 
productivity increase. Conversely, more health risk factors lead to performance and productivity impairments.

The relationship of risk factors and cost improvement is also an inverse one. Higher health risks lead to more deficiencies and thus to a worsening of the cost situation. Several studies confirm this relationship: Grobe et al. (2011); Sockoll et al. (2006); Dugdill et al. (2008); Meijster et al. (2011); Laaksonen et al. (2009); Van Strien and Koenders (2010); Köper et al. (2009); Hausknecht et al. (2008); Vu-Eickmann et al. (2017); Walter, Plaumann et al. (2011). Thus, risk factors and performance are interrelated in a twofold way: Once directly and indirectly through the cost improvements.

The impact of risk factors on leadership ability has an inverse direction, too. Risk factors lead to disturbances, fluctuations, and regulatory impediments which can complicate successful management behaviour. The more risk factors, the greater the risk of failure that affects the physician's ability to lead. To deal with this connection and the frequently incapacity of physicians with "incidents" and to handle personnel development decisions, Majumdar (2004) shows in his analysis the management and management behaviour of general practitioners.

The impact analysis in Brooke's model of absenteeism (1986) confirms the relationship between emotional well-being and physical health. 'Satisfaction' as a partial aspect of well-being improves the 'health status'. In occupational health literature, there is widespread evidence that low job satisfaction precedes reduced physical and mental health. (House, Wells, \& Landerman, 1979; Jenkins, 1971, 1976; Kasl, 1973; Locke, 1976; Martin \& Schermerhom, 1983; Sales \& House, 1971 - cited in Brooke, 1986). It has also been shown that dissatisfaction is associated with increased alcohol consumption (Trice \& Roman, 1978, Trice, 1980, Markowitz, 1984 - cited in Brooke, 1986).

The impact analysis of the psychological contract, as shown in Figure 7 confirms the relationship between HR practices and performance (Katou, 2013). Since health management is one of the HR practices that increases physical health and emotional well-being, it can be assumed that a relationship with the increase in performance is also confirmed here (via reduction of the risk factors).

\subsubsection{Improvement of productivity, quality and profitability}

The three sub-goals of this area influence each other as follows: An improved quality and customer focus, according to Meijster et al. (2011) and Amend (2005), promotes the performance and productivity of the surgery. So Marcks $(2012$, p. 7) recognizes that all levels of an organization on the quality management are involved and responsible, but the management must promote it and live it as a role model. "Quality is clarity and intelligent leadership" (Müller and Schwarz (2010, p. 114) - cited in Marcks (2012, p. 7). The leaders of a general practitioner's surgery need to take personal responsibility and a role model here (Gerlach (2001) cited in Amend 2005, p. 
73). The reduction of costs, that means the cost improvement, leads directly to an increase in performance and productivity.

An improvement in the cost situation, which is usually associated with an increase in performance pressure, has a negative effect on emotional well-being due to the resulting "cost pressure". (Goetz et al., 2015)

The last-mentioned compound in the causal model of absenteeism (Brooke, 1986) is not further discussed. There, 'pay', a component of the cost situation, is hypothesized as a determinant of 'satisfaction', an indicator of emotional well-being. However, there is only one relationship assumed in the sense that an increase of 'pay' leads to an increase of 'well-being'. The side effect, which leads a higher pay in the form of pay and rewards also to a higher pressure to perform, i.e. a deterioration of mental health, remains unmentioned.

An analysis of the psychological contract using the contract promises fulfilment indicators as shown in the framework of Figure 7 (Katou, 2013), reveals no further crossimpacts to be considered.

\subsubsection{Deriving priorities from the literature review and cross-impact matrix}

The literature review of the previous sections is used to build the cross-impact matrix as a table as follows (see table 2). In the first column and in the first line are the goals of health management proposed by Walter (2007). The direct influence is entered in the respective table field: 0 - none, 1 - low, 2 - medium, 3 - strong). Thus, the entry " 0 " in the line "Leadership ability" and the column "Reduction of risk factors" means that neither in the literature search itself, nor in the causal model of absenteeism (Brooke, 1986) or in the psychological contract framework (Katou, 2013), there is an indication that changes in "Leadership ability" have a direct impact on "Reducing risk factors" (but indirectly through emotional well-being or improving physical health). A small influence ("1") was entered if a literature, model or framework note was found. Accordingly, the influence strength " 2 " and " 3 " were awarded. A " 3 " was awarded only if a direct relationship was documented in the literature, as well as in the causal model and in the physical contract framework.

The derivation of priorities from the cross-impact matrix is carried out according to Vester (2009) using active and passive sums. The active sum (AS) is the sum of all direct influences, or in other words, the row sum - highlighted in light green in the table. The passive sum (PS) is accordingly the number of incoming effects - or in other words, the column sum - highlighted in light yellow in the table. The active sum shows how much changes in the element affect the other elements in the overall system. An activity sum shows the force of influence. The passive sum, on the other hand, shows the influence of an element by other elements (influenceability). 


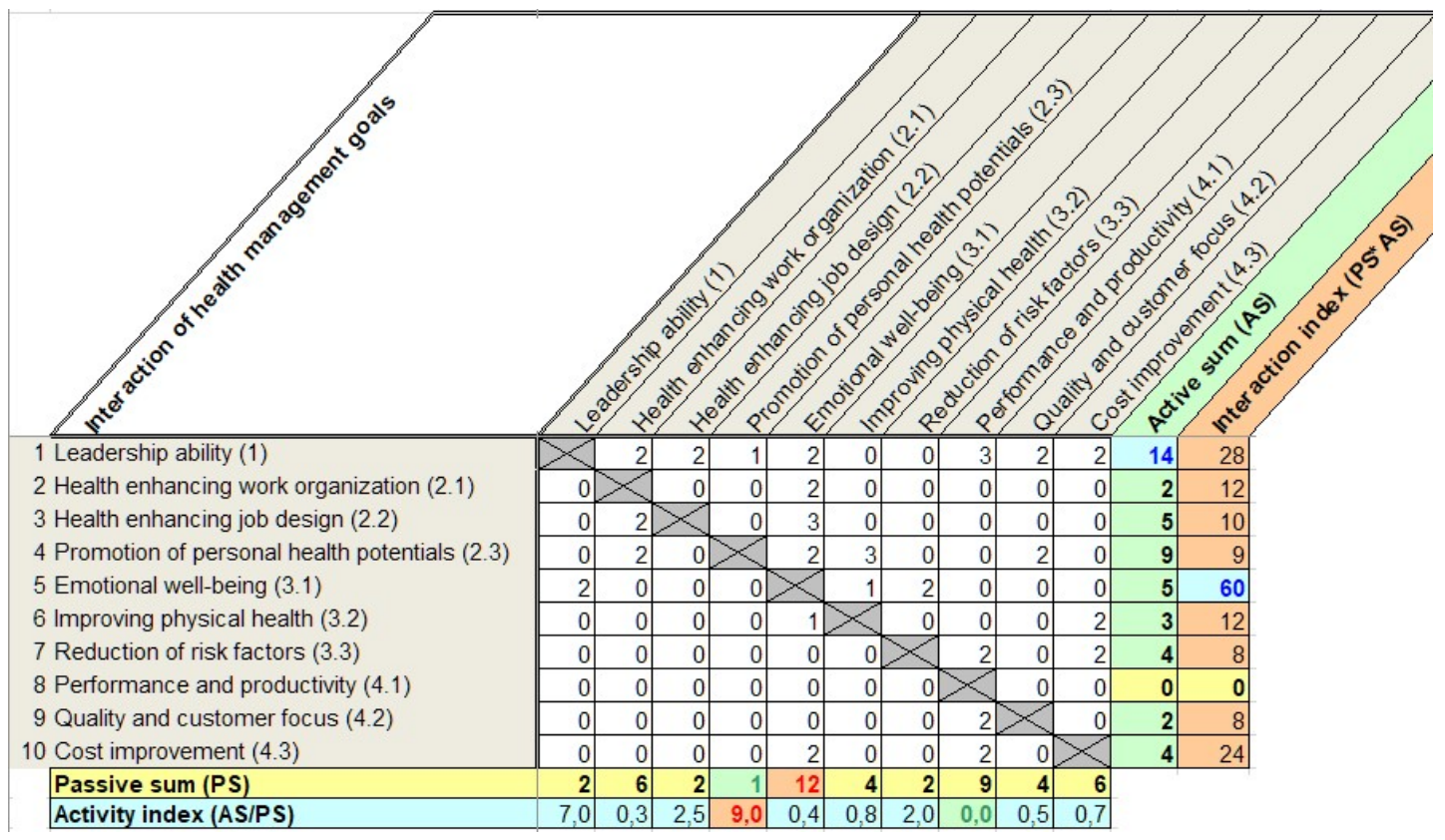

Table 2: Cross-impact matrix of the interaction of health management goals

The element with the largest active sum (14) is clearly "Leadership ability" - highlighted in the table by blue writing - followed by "Promotion of personal health potentials" (9). The highest passive sum (12) clearly shows "Emotional well-being" highlighted by red lettering - followed by "Performance and productivity" (9).

The active and passive sums are used to find out the changes with the largest control lever; the so-called "steering abilities" (Gomez and Probst, 1997) For this purpose, the elements are arranged and grouped in a fourfold table from which a priority list can be derived (Wilms, 2012).

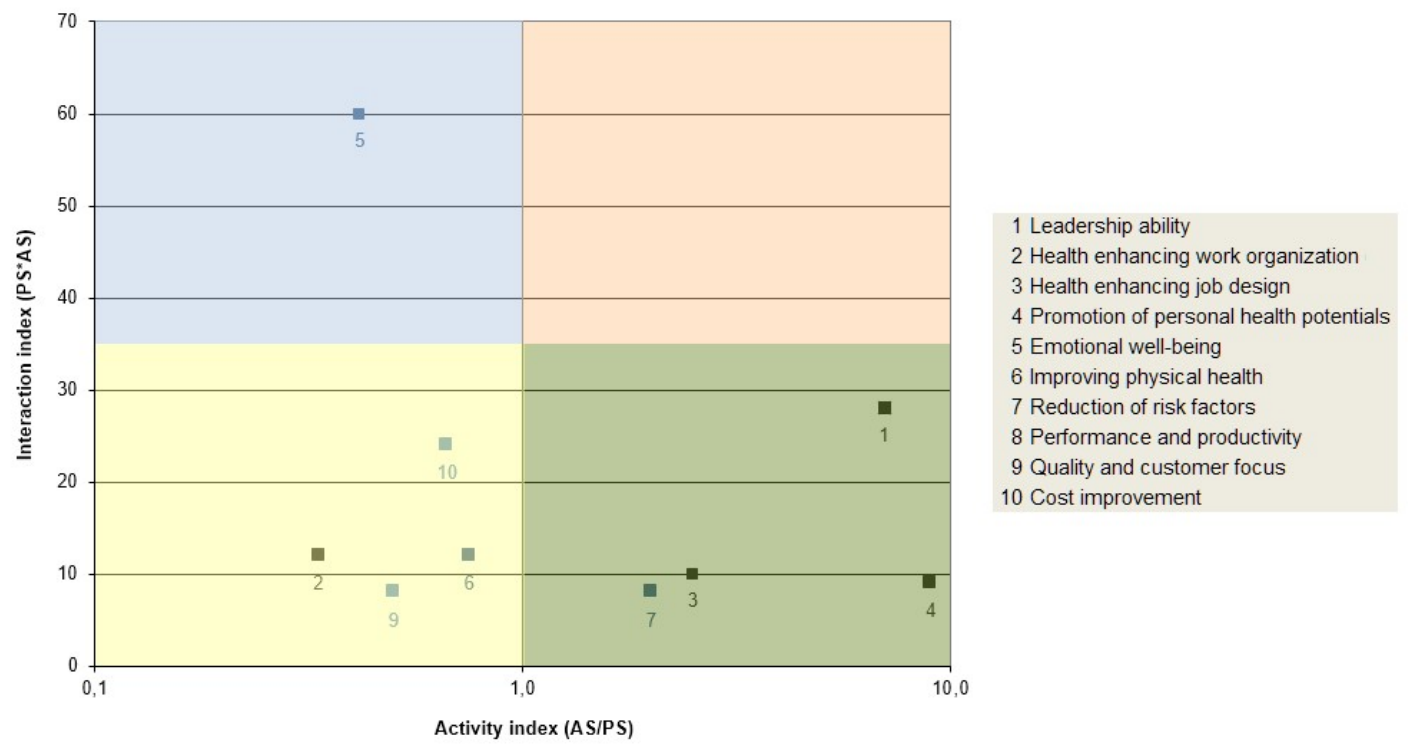

Figure 10: Fourfold table for identifying priorities in the control levers 
For this purpose, two additional key figures per element are determined in addition to the active and passive sum, the activity index and the interaction index. The activity index (AS / PS) is the quotient of the active sum (AS) and passive sum (PS). If it is greater than 1, this means that the influencing strength outweighs. In the above table, the activity index is shown as a light blue line. The interaction Index (AS * PS) is the product of active and sum. It shows how strongly the element is integrated into the dynamic and network. In the above table, the interaction index is shown as an orange column.

Following Vester (2009), Gomez and Probst (1997), and Wilms (2012) for evaluation purposes, the activity and interaction index are arranged in a fourfold table as in the figure above. In the horizontal direction, the fourfold table shows the strength of the influence. The further to the right an element is, the greater its activity index, an indicator of the influence strength. In the vertical direction, the fourfold table shows the interaction index from the top downwards. The further down an element is, the lower is its interaction index. If low or high intensity of influence are combined with low or high interaction, four subpanels are obtained for the grouping of elements. The name of an element is identifiable by its number in the list on the right-hand side. This classification leads to a classification of the elements which is relevant to the action (in brackets the colouring in the fourfold table):

- Passive: The element affects below-average other elements and is also influenced below-average by other elements (low - low / yellow).

- Critical: The element has an above-average influence on other elements and is also above-average influenced by other elements (high - high / red).

- Buffering: The element influences no other elements or only in a very small degree. On the other hand, it is highly influenced by other elements (low high / blue).

- Active: The element influences others in a strong way, but itself it is not or only in a small degree influenced by the others (high - low / green).

Particularly promising changes should be made with a predominantly active element (coloured green in the fourfold table). In the health management system of a general practitioner's surgery, this would be the leadership ability, the personal health potential of MFAs (prevention), a health enhancing job design and the reduction of risk factors.

Within the active elements, the so-called main or steering levers, the promotion of personal health potential is the goal with the strongest side-effects. Measures to promote personal health potentials (prevention) at the same time support other health management goals; this is the reason why in the framework of an integrated health management system for general practitioners' surgeries, this research focuses on improving the personal health potential of MFAs. 
The second priority would be to improve leadership ability. But because a general practitioner who is interested in implementing a health management system for his practice can hardly be recommended to improve his or her own leadership ability first, this should not be the first step. Compared to promotion of personal health potentials (prevention), measures to improve leadership ability also have the disadvantage that side effects and repercussions must be expected, the effects of which are more difficult to control.

If the proposed prevention measures are not convincing and the physician does not want to further develop their own leadership qualities, measures for health enhancing job design would still be worth considering. The same applies to measures that directly affect the reduction of risk factors, such as ergonomic workplace design.

\subsection{Summary}

In this chapter, primary prevention in German general practitioners' surgeries was discussed using the following models, concepts and theories:

- A model of employee attendance (Rhodes and Steers, 1978)

- A causal model of absenteeism (Brooke, 1986)

- Recommendations for implementing a company health management system in SMEs (Walter, 2007)

- An exploration of small firm psychological contracts (Atkinson, 2008)

- A HR practices - psychological contract - organizational performance linkage framework (Katou, 2013)

Based on these models and related literature, a cross-impact matrix was created from which an order of steering levers was derived. These are:

- Promotion of personal health potential of MFAs (prevention)

- Improvement of the leadership ability of general practitioners

- A health enhancing job design

- Reduction of risk factors

For the strongest lever, the prevention, in answering the questions

- How to prevent musculoskeletal disorders?

- How to prevent mental disorders at the workplace?

- How to prevent the respiratory system at the workplace? 
- How to prevent injury at the workplace?

- How to promote general health and well-being at the workplace?

peer-reviewed articles have been evaluated for the preparation of the employee survey.

The literature review found that the following areas can be considered as little explored (under-researched):

- Human resource management in micro-enterprises

- Psychological contract in micro-enterprises

- Health management in general practitioners' surgeries

- Implementation of health management measures 


\section{Methodology}

This chapter will discuss the researcher's approach to find answers to his questions about the thesis, as well as aiming to develop a suitable decision-making tool which enables physicians who have employees to decide which activities are valuable to improve the health management of their medical office.

At first, the author's research paradigm will be discussed, followed by the ethical considerations, which are of importance and relevance to the research, the planned research questions, the questionnaire design, the way in which the targeted group of employees could be reached, the sampling, the role of the researcher, and how the results will be analysed and weighted. Furthermore, the role of the researcher and ethical considerations with regard to potential risk of 'confirmation bias' as well as issues of 'socially desirable' responses will be discussed.

The results of the literature reviewed are secondary quantitative data concerning illnesses and absent days, which are the basis for calculating the influence from health of employees to the profitability of organisations. Additionally, there are studies, which explore the view of the employee and their experiences linked to company success.

\subsection{Research paradigm and research philosophy}

\subsubsection{Research paradigm}

A paradigm is a shared world view that represents the beliefs and values in a discipline and that guides how problems are solved (Schwandt, 2001).

In the following, I will try to see occupational health management among four different paradigms proposed by Burrell and Morgan (1982, fig. 3.1). Each of the four paradigms stands alone and creates separate ways to analyse organizations and societies. The four paradigms result from the combination of two dimensions. On the one hand there is the dimension Objectivism vs. Subjectivism (nature of science) and on the other the dimension change vs. stability/regulative (nature of society).

An objectivist's view of the world assumes that social entities (such as hierarchies, departments, groups, customers, ...) exist in reality, they are "objective" present and have structures and processes that support their adaptation and / or goal achievement. They exist independently of the respective actors and are in their views external units to which them faces themselves as objects and in which they move.

A subjectivist's view of the world assumes that social phenomena arise from the perceptions and successions of actions of social actors. Therefore, they do not exist objectively / real, rather they are individual constructs of individuals, groups or organi- 
zations. Similar phenomena, situations and interactions are perceived differently and lead to different reactions.

According to Kelemen and Rumens (2008, p. 22), the dimension of the "nature of society" is best understood through the following two questions: "Is society (or the ideal of society) a stable, orderly and coherent entity (an output)? Or is it always in a state of flux and transformation (a process), on its way to being constituted but not quite there?"

If the first question is affirmed by the researcher, it can be assumed that the regulative perspective is taken. The researcher is looking for explanations of how and according to which rules a social unit is organized and what gives it stability. The affirmation of the second question marks the radical change. This perspective is more about recognizing how change is happening and what makes the transition from one state to another. The dynamics and process orientation are in the foreground.

The combination of the dimensions "nature of science" and "nature of society" leads, according to Burrell and Morgan (1982), to four prototypical research paradigms.

Radical humanist: - subjectivist and change-oriented - Researchers who see this paradigm as a model for their actions have a process-oriented view that places the individual's own world view at the centre. The subject is seen in all facets holistically in a complex structure of action. Occupational health management would be seen as a process that takes into account all factors influencing individual health and personal well-being, including out-of-work and family life, biography, social environment, genetic disposition. Health and well-being themselves are understood as processes dominated by psychosomatic, salutogenetic and alternative medicine. The more situational recommendations for action to promote health potentials have to be highly adaptable and flexible, they "bring the individual from where it stands." Decisions are more emotional and sometimes show irrational characteristics.

Radical structuralist: - objectivist and change-oriented - This research paradigm, too, is committed to a process-oriented view. The world view is characterized by seeing also organizational units and social units as living organisms, comparable to biological systems, where change and adaptation processes are an integral part of the basic structures that are also designed for these requirements. Structures and processes are objectively given; they must be "only" recognized, understood and applied by the researcher. The general orientation is the learning organization, which ensures, as in a cell renewal process, that the organism survives permanently. For occupational health management, this means that the concept of health extends beyond the individual to "healthy leadership". Not only the physical, mental and social well-being of the individual is seen as the basis of organisation's performance, but involvement of health and well-being in a more comprehensive process of adaptation and change. 
Interpretivist: - subjectivist and statically, regulatively oriented - A research with this paradigm focuses on the corporate and organizational culture. In order to understand this, which can hardly be grasped objectively, the researcher has to make tangible the daily interactions and their meaning. Often this can only succeed if the researcher incorporates her/himself into organizational life in order to facilitate a better interpretation. This is the only way to know which rules (and rituals) determine the daily flow of activities. Following this guiding principle, this means for the occupational health management, the challenge to develop a "health and well-being culture" and to maintain it permanently.

Functionalist: - objectivist and statically, regulatively oriented - Researchers who develop theories according to this paradigm see, at least from their point of view, the world as "it is" (Kelemen and Rummis, 2008, p.23), insofar they are objectivistic. Using quasi-scientific methods, they try to recognize regularities (natural laws) in social units and derive rationally based recommendations for organizing them. Saunders et al. (2012) see this paradigm as the most commonly used in business and management research. The guiding principle is to understand organizations as complex machines, which are initially seen as a black box and then, thanks to research, become a white box. Occupational health management complies with this paradigm in the interest of achieving an overall goal. The "Health Competency", which is based on occupational medicine, organizational psychology and sociology, is used to investigate recommendations for action to maintain and improve the performance of the entire organization.

If one follows the statements of Burrell and Morgan (1982, fig. 3.1) then the paradigms are "... mutually exclusive. They offer alternative views of social reality, and to understand the nature of all four views of society. They are contradictory, being based on at least one set of opposing meta-theoretical assumptions. They are alternative, in the sense that they can operate in different paradigms sequentially over time, but mutually exclusive, in the sense that one cannot operate in more than one paradigm at any given time in time, since in accepting the assumptions of one, we defy the assumptions of all the others ". Following this reasoning, I decided to build up the research as a functionalistic researcher.

This paradigm seemed to me to be the most appropriate for the upcoming problem to develop a priority concept for health promoting measures for German general practitioners' surgeries. With "rational explanations offer solutions to rational problems", Saunders et al. (2012, p. 142) outline the attitude that I found to be the most appealing compared to other paradigms, and which most corresponds to my personal organizational and managerial understanding. The literature analysis (Chapter 3), which only allowed measures with demonstrable results for further development, and the elaboration of a comprehensible decision-making concept emphasize the rational problem-solving approach. In addition, in my opinion, physicians' practices also see themselves as functional units in a social system, which is why this paradigm is expected to be highly accepted. 
As will become clear in the following sections, the acceptance of recommendations for action is in the foreground. The recommendations for action are based on operational "health competence". Decisive for the implementation, thus the way from the knowledge to action as argued by Mandl and Gerstenmaier (2000, eds.), is the acceptance, which represents an indispensable condition.

For the selection of the research paradigm, reference should be made to the conclusions of Fazliogullari (2012)in his article "Scientific Research Paradigms in Social Sciences": “The question of 'which paradigm is the best?' is not a meaningful question since there is not any answer of it. For this reason, it is all in vain to compare the paradigms according to a criterion. Every paradigm has a coherency and consistency in itself. These ideas require normally that multiple paradigm approach is recognized. Multiple paradigm understanding, on the other hand, will mean increasing paradigms day by day." (Fazliogullari, 2012 p. 52)- Or to put it another way: selecting a paradigm is based on a selected paradigm.

The selected paradigm characterizes the ontological aspect of the research philosophy by describing with what assumptions we would like to see the nature of reality. Furthermore, epistemology, axiology and methodology are characteristic of different research philosophies according to Patton (2002) and Saunders et al. (2012).

\subsubsection{Epistemology}

While ontology raises the question of the nature of reality, epistemology focuses on the question of the nature of recognition (realization). So, the question of how we acquire our knowledge and what we believe to be true. Basically, four philosophical directions can be distinguished: positivism, realism, interpretivism and pragmatism.

In positivism "only observable phenomena can provide credible data, facts. Focus on causality and law-like generalisations, reducing phenomena to simplest elements. " (Saunders et al., 2012, p. 140). Related to health management, a hard-core positivist measure would mean that only the number and percentage of sick leave days counts as an observable, trusted date and fact. (Again, relying on a positivist world view of the diagnosing physician.) Exceeding phenomena such as "well-being" or "stressed", "burdened" or "motivated" are strictly speaking not measurable or directly observable. It depends on indicators and statistical data that "suggest" a phenomenon. The same is true of causality and generalizations. There is no positivistic proof of a law-like generalization regarding the relationship between "healthy eating" and "reduction of absence". This is where the difficulties begin to determine what constitutes the beneficial effect of a specific nutrition concept on a single person.

Realism also assumes that only "observable phenomena provide credible data, facts." (Saunders et al., 2012, p. 140). However, direct realism assumes that it is theoretically possible to explain phenomena through reliable knowledge of facts and contexts. The main difficulty is that we only have inadequate information 
leading to inadequacies and inaccuracies in our observations. If we were to take the example above, if we would only have sufficiently accurate records of a person's daily intake of food, then we could establish an association with absence days - the assumption of direct realists. By contrast, critical realists assume that the available data only lead to misinterpretations, because our sensory experiences in the observations can also deceive us. They therefore accept the ideas existing in the respective environment and context as given and seeks explanations based on these circumstances. The terms of primary prevention used in health management are a good example here. The preventive effects are unobservable and hard to pin down, but they are taken as true in the context.

Interpretivism sees, as shown above, as a starting point for the acquisition of knowledge "subjective meanings and social phenomena. Focus on the details of situation, a reality behind these details, subjective meanings motivating actions " (Saunders et al., p.140). To take an example from health management, it is not so much which objectively ascertainable factors speak for a high workload, but rather to what extent the individual employee feels "burdened" - or the so-called "perceived burden" is decisive in this basic epistemological philosophy. Also, the widely held belief that physical fitness leads to increased performance is a typical social phenomenon that corresponds to an interpretive understanding of health management. An interpretative approach is also to see the entire organizational environment in which health management takes place.

Finally, for the pragmatist, observable phenomena as well as subjective meaning can lead to useful knowledge. The focus is on the application, feasibility and usability in practice. The researcher is "integrating different perspectives to help interpret the data" (Saunders et al, 2012). In occupational health management, this becomes clear when planning health-promoting measures. In order to learn more about the effectiveness of a measure, as far as possible in the sense of a critical realist, one bases oneself on studies on observable phenomena. When it comes to the implementation of a measure, the subjective meaning (acceptance-perspective of the employee) in the sense of an interpretivist becomes more important.

The epistemological aspects of the research philosophy of this research are most closely related to the pragmatist. Like Shields (1998, p. 197), the author sees pragmatism as the philosophy of common sense, as actions are judged in the light of their practical consequences and usefulness. According to the pragmatist standpoint, theories are used as tools by exploiting them as a conceptual framework, as proposed by Shields (1998, p. 202). This bridges the gap between theory and practice.

The conceptual framework guiding this work is the HR practices - psychological contract - organizational performance linkage framework proposed by Katou (2013) (see Figure 7). According to the common-sense aspect, the mutual expectations of the employment relationship are forming the focus. They decide on possible performance improvements through health management measures. The framework serves 
as an overall picture and is used to discuss and derive ideas. This framework considers both the interpretivism's / constructivism's view of the psychological contract (see also Atkinson 2005, pp. 60 ff.) and the perspective of realism as expressed in the fact sheets of the CIPD recommendation (2008) and its statistical evaluation (Katou, 2013). The research questions themselves can be anchored in this framework and help to clarify the conditions under which options for measures on health management are particularly promising.

The first research question on the studies (question 1), which prove a connection between health-promoting measures, combines the input of the framework (HRPractice) with the output (organizational performance). According to the utility claim of a pragmatist, general practitioners' surgeries can only be recommended for measures that have already proven themselves in other environments and preferably in small companies.

The research question on employee preferences (question 2) addresses a relational, employee-related input factor in fulfilling the promise of the psychological contract. At the same time, it touches on the implementation aspect that is so important in the practical implementation that the employers should know the expectations of their employees. For a specific environment (general practitioner' surgery) and a specific context (primary prevention), a preference profile is researched and checked for its truth content with hypothesis tests.

The research question according to a priority concept (question 3) follows a pragmatic principle, one in that it integrates the "objective" studies (effectiveness) with the "subjective" expectations (acceptance) and further perspectives (cost level) and converts them into a usable result.

The research question on the adaptability of the priority concept in one's own practice (questions 4 and 5) deals with the implementation aspects that are important for the practical realization. In addition, the development of an adapted new framework, which is tailored to the requirements of a general practitioner practice, is specified and discussed.

\subsubsection{Axiology}

The axiology of a research philosophy describes the relationship between the research activity and the values of the researcher and how they may influence each other. Values can influence research questions, whereby already the selection of a question can be understood as a valuation, because with the selection the question is given an importance and not to other questions, which are not asked. Values thus influence the acquisition of knowledge and thus determine its trustworthiness. They determine the research action and what we accept as "true".

According to the different research philosophies axiological basic positions can be distinguished: 
The positivistic research ideal strives for research in a value-free manner. It regards the researcher as completely independent of the phenomena to be observed and assumes that objective distance is always maintained.

Realistic research accepts that research can hardly be free of value judgments. Wanted or unintentionally, it leads to distortions that influence the research results. The focus is therefore to identify and disclose possible sources of bias and to make precautions for minimizing the effects of bias.

The interpretivist assumes that there is no value-free research. The researcher is always an integral part of what is to be researched and cannot be seen independently of the research situation. The results are always value-bound and subjectivist.

In pragmatism, after all, the meaning of values is not so much the research situation itself, but rather the interpretation of the results, which takes both an objectivist and a subjectivist perspective.

This research corresponds most closely to the pragmatism approach. Subsection 4.4.3 - The role of the researcher and ethical considerations - addresses axiological aspects in more detail. In particular, it becomes clear to what extent the confirmation bias and the social desirability could play a role and whether they actually play this role.

\subsubsection{Methodology}

The methodological choice characterizes the research design, i.e. the plan according to which the research questions are to be answered. As explained in subsection 4.1.2, the pragmatism's research philosophy is most relevant to each research questions. Accordingly, a mixed method simple approach was chosen, as described by Saunders et al. (2012, p. 165) applied in a two-stage research design.

Section 2.2 "German general practitioners' surgeries and health management", in the manner of a descriptive study, discusses the decision-making environment of a general practitioners' surgeries and the special links between MFAs and primary prevention measures.

Chapter 3 sees literature review as part of exploratory and explanatory research. In the exploratory part, the basis for questioning the MFAs is created. The explanatory part focuses on the relationships between primary prevention and absence and deriving priorities for German general practitioners' surgeries. In addition, a more comprehensive process and causal model as well as a linkage framework are used to clarify attendance and absence as parts of a comprehensive theory.

The main research strategies used in this study are surveys, questionnaires as well as structured interviews. In first stage the questionnaires not only follow a purely quantitative approach that allows for hypothesis testing but also provide room for qualitative answers. In the decision model for developing a priority concept are introduced 
further qualitative aspects, which are then in second stage compared using structured interviews.

The two following sections discuss individual methodological questions on the MFA survey, the development of the priority concept and the GP interviews on implementation.

The improvement of health management in general practitioners' surgeries, especially concerning the personal health potentials (primary prevention), is linked to the support of the proposed health program by the MFAs. The perspective of the employees and their support of activities to improve health management are essential. Without the support of MFAs, an improvement is impossible or at least very difficult. However, to answer the research questions it is important to think about the results in different perspectives. The point of view combined with a weighting of priorities is important to provide an adequate result. To find the answer, an approach, which respects the different criteria and interests of different stakeholders, was needed. The approach of multi-criteria decision-making as outlined in Figure 2 provides the possibility to generate decisions which provide a ranking of different activities. There is not one answer to the question how the health management of the employee in practitioners' surgeries could be improved but there is a list of activities which could lead to this improvement.

\subsection{Methods for questionnaire design}

In this section, the development of the questionnaire is described. All questions are the result of the literature review. The pro and cons of each possible question are explained. Finally, ten questions with sub-questions had been developed/formulated, including multiple-choice questions and open questions.

Within the framework of the multi-criteria decision-making model, the objective and task of this section is to represent, from the set of possible alternatives, those who are narrowly selected for a general practitioner' surgery (see emphasis in Figure 11).

The questionnaire covers health management activities with a positive effect on disease prevention, and the potential to reduce the level of absent days. The activities were identified in the literature review.

Most of the questions are multiple-choice questions. There are open questions to enable the employee to give a free feedback without the restriction of multiple-choice questions. This could generate data on health management activities which were not identified in the literature research. Additionally, the open questions were used to provide the participants a possibility to put in their opinion without the boundaries of multiple-choice questions. This qualitative data has also been used to check if the main wishes or problems of the MFAs have possibly not been represented in the questionnaire. However, the results from the combination of the multiple-choice 
questions and the open questions has been used to generate quantitative data which were used for the multi-criteria decision-making.

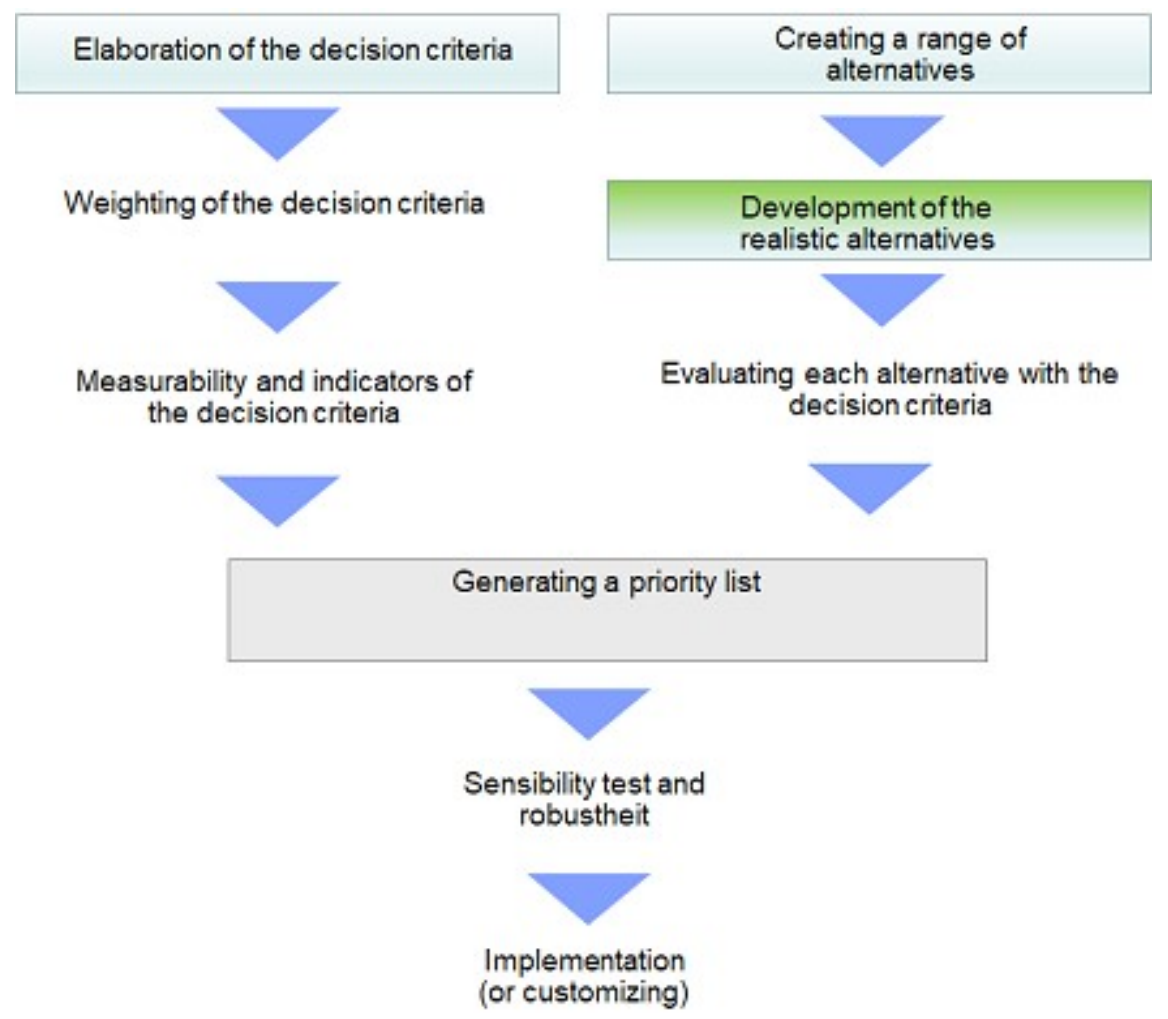

Figure 11: Questionnaire design as part of the multi-criteria decision-making model

The participants of the questionnaire survey are employees of general practitioners' surgeries, the MFAs and all other persons who work as medical assistants (cf. 2.2.2).

The research "Monitoring and Evaluation of Worksite Health Promotion Programs Current state of knowledge and implications for practice" (Engbers, 2007) recommend the usage of short and clearly understandable questionnaires to achieve a higher return quote. The questionnaire should be fillable within five to ten minutes to avoid aborting when filling. The goal of the author was to keep the barrier for the participant as low as possible and to motivate the participant to complete the questionnaire.

In the following text, the translated questions are highlighted by a grey background and indention. In Appendix 3 the original German questionnaire is documented. The possible answers are written in italics. 


\subsubsection{Questions about demographic characteristics}

The questionnaire starts with personal questions concerning the participant.

At first the data on the age and gender of the participant should be generated. The questions for gender and age will be the first information about the participant. The estimated level of female workers is high. The quote of female medical assistances in general practitioners' surgeries is $98 \%$ (BMG: Healthcare facts, 2016, p. 97). However, the results for this question will confirm this quote or provide another quote.

The first question in the questionnaire is:

In a multiple-choice system, the answer can be female or male. Since over $98 \%$ of the MFAs are female (cf. 2.2.2), this question is more of a control function with respect to the representativeness. It was not to be expected that any improvements in health management could be made by the answers.

The second question is:

The possible answers in the multiple-choice system are:

$$
\text { O } 18-25 \quad \text { O 26-35 } \quad \text { O 36-45 } \quad \text { O 46-56 } \quad \text { O 56-65 } \quad \text { O }>65
$$

Since the education of MFAs is not completed before the age of 18, employees under 18 years are not included in the questionnaire and therefore there is no possible choice.

There are no studies on the age structure of the employees of general practitioners' practices. The evaluation of the answers is therefore of interest in the following respects:

- In Germany, training for MFA is one of the most popular career aspirations for young women. Since 1996, primary prevention has been part of the content of vocational training (Bundesgesetzblatt, 2006). Therefore, it is of particular interest to analyse possible differences in the responses of the different age groups to determine whether they have different expectations and preferences on possible actions.

- One of the two focuses of the German national prevention act (cf. 2.1.1) is on the demographic development. It is therefore of particular interest to investigate whether there is any problem with "aging workforce" in general practitioners' surgeries.

- Do the different proposed prevention measures differ in their preference by MFAs differently for each age group? 


\subsubsection{Questions about the general practitioner's surgery}

These first two questions were focused on the employees and personal information about them. The next question has the focus on the general practitioner's surgery.

To be sure, that there is no focus on a special area like dermatology there is an open question for this kind of surgery.

The structure of medical practices a MFA can work for should be the same regardless of the medical speciality. However, the medical specialisation of the physicians is necessary to keep the focus on the target group and to avoid mixed groups, since the focus of this research is on MFAs in general practitioners' surgeries.

Regarding the measures to be proposed, it is important to know what the focus of the practice is, since the general practitioners' surgeries have the following specific characteristics which could lead to a bias in the answers:

- The issue of "vaccination" has a different significance for general practitioners' offices, since contacts with infectious patients are much more frequent (2.2.6)

- Primary prevention as a possible task component and object of a practice offer has a different meaning than in specialist medical practices (2.2.3).

To this extent, this question serves as a question of control to be able to remove or eliminate answers from general practitioners.

After the information about the general practitioners' surgeries the questions move in the direction of health management and the possible health management activities.

The answer can be "yes" or "no" followed by an open question: "If yes, please describe which, if no describe why not:"

The data on existing health management programs is useful for the future implementation of activities and the general acceptance of health management.

The question is about existing health management programs in the practitioner surgery. This question is a combination of multiple-choice and open questions. This question design is used to get a free feedback about every activity of health management which is practised. A multiple-choice question using a list of health management programs would exclude health management programs, which are not listed. Plus, the participant can provide activities which are not identified by this research.

After the question about the health management status the question is about the general acceptance. 
The answer can be "yes" or "no" followed by an open question: "If yes which? if no please explain why not:"

The general acceptance of health management programs and activities is important to implement health management. If an employee does not want any activity to improve the health management, it is much more difficult to improve the health management. Most activities, which were identified in the literature review with a proven effect; need the support of the employees. If the participant supports such activities and commits himself, the rest of the questionnaire is relevant.

If this question is denied the open question should help to learn more about the why. There can be several reasons for a "no". E. g. the MFA does not like such activities or mistrusts the employer, or she thinks that the employer has in principle no right to interfere with her private life through primary prevention measures. This qualitative data can help to understand the background of the MFA if the program is denied

\subsubsection{Questions about the promotion of personal health potentials}

After the critical question about acceptance of health management, the focus of the questionnaire is put on the personal health of the MFAs. The quality of the questionnaires is strongly linked to the quality of the content on which the questions are built. There are many contents which could be used in the area of health management. The literature review was used to narrow down the area of the research and to select which information is useable for the research and the questionnaire.

The idea of the questionnaire is that the employees of general practitioners' surgeries can choose between different activities they would personally prefer and accept to improve the health management in their organization. The activities, which are provided to the employees, must have a proven result on health. However, these activities must lead to a reduction of absent days of employees.

As measure for the decision which activity is used in the questionnaire, the effect of the activity on the absence days is relevant. However, following the research question and the results of the literature review the measure of absent days is most appropriate and workable for the research. Following this, the activities in the questionnaire are structured by the number of caused absent days. This means the diseases with the highest rate of absent days are the main topics with the highest potential of reducing absent days. The goal of health management is to prevent disease and reduce absent days. The sequence of the points is based on the number of absent days caused in average at this area (Grobe, Dörning et al., 2011). An additional criterion for the provided activities is that they are economically useful, and it must be proved that it has a medical result which is significant. This is ensured by the evaluation of the studies from the literature review. Using the framework from Bräunig et al. (2015) the occupational illness days are categorized in the following five areas, which could be included in the questionnaire. 
1. Prevention of musculoskeletal disorders

2. Prevention of mental disorders at the workplace

3. Prevention of diseases of the respiratory system at the workplace

4. Prevention of injury at the workplace

5. Promotion of general health and well-being at the workplace

The area of occupational accident and injury prevention at the workplace was not included. Work safety is important for a good health management; however, this area is mainly part of work security for industrial works and is strongly regulated by law. The general practitioners' surgeries handle this area with the help of quality management. This includes e. g. stab wounding from needles, which is dangerous because of infectious diseases that could be transferred (2.2.6).

Goal of the questionnaire is to find out what the medical assistants who are employed in medical offices would most likely accept and integrate in their daily work. The questions need to be simple and clear on a non-academic level to avoid false answers caused by misunderstanding.

\section{$\underline{\text { Prevention of musculoskeletal disorder }}$}

This includes activities for the universal support of health, which is very broad, and not clearly structured. In this area, all actions which can help to prevent illness were considered. It is divided in to seven subgroups and the International Statistical Classification of Diseases and Related Health Problems ICD10 is used as framework. In the literature review, several studies proved that programs for physical movement have a significant positive influence on the employees' health. Brewer et al. (2006) found high quality studies that examined the effects of interventions in the office on musculoskeletal health, this is underpinned by Melhorn and Gardner (2004) who give an example for the caused costs for the national economy of the USA. Abraham and Graham-Rowe (2009) found out that programs for more physical activities which were developed after 1997 and include pedometer help to improve health management. The focus on movement with a pedometer is four times as effective as other activities. This was confirmed by Dugdill, Brettle et al. (2008). Barr-Andersen (2011) (Barr-Anderson, 2011)found out that "short activity-boots" during work time bring a moderate but significant effect.

Following these studies, the focus of the following questions was necessary to find out which activities the MFAs would like to do. The main question is: 


\section{- Sponsored activity after work}

The rating scale for the answers are:

total acceptance tendency to yes neutral tendency to no absolutely no

The answer options for each question is effectively a Likert's scale (1932) and later on the statistical significance will be calculated (cf. 5.2.3). However, the focus of these questions is how to improve the movement and which activities would be mostly preferred.

\section{Prevention of mental disorder}

Mental illnesses cause second most absent days and it is a growing problem (Grobe, Dörning et al., 2011). In the literature research, there are many studies about mental health. However, there are no articles, which consider the linkages between mental disorder and occupational prevention in the service sector or in general practitioners' surgeries. At the German congresses of occupational medicines, the themes mental disorder and burnout were discussed very intensively, but the number of relevant studies is low. Mental health is important; especially the area of depression but there is a gap in the literature on which activities could be done by an organisation to avoid these mental illnesses. There are several studies about prevention of mental illness, but the literature review did not identify researches which have the focus on prevention within the work time. The goal of this research is to provide suggestions for improving the health management; therefore, the author needed activities, which can be implemented in the day-to-day work, and their usability and benefit must have been proven. However, this area is an upcoming problem in the occupational medicine and causes many absent days. Nevertheless, without more knowledge about it the author is not able to implement mental health in the questionnaire. To ask employees with an open question, how their mental health could be improved is difficult because this suggests that they have mental problems. Even a question about the status, like "Do you have absent days because of mental disorders" does not help to answer the research questions, because its results cannot be transferred into an activity. There is also an ethical dilemma, because mental health is seen by society that people are 'crazy' and possibly not fit to do their job. Nobody will admit that they are depressed or on an anti-depressive medication because it is documented in their medical records, and for working in a general practitioner's surgery it might not look good and may even lead to further problems with the employer. 


\section{Prevention diseases of the respiratory system}

There are no results for the service sector and prevention of illness of the respiratory system. For industrial workers in coalmines or plant workers the health of the respiratory system and the prevention of lung problems are important. In medical offices, there is no higher risk for illness of the respiratory system caused by the work itself.

This is a multifarious area and the author believes that there are possible activities to prevent illness of the respiratory system, especially in the linked areas likes flu vaccine or inflectional disease. For example, pulmonary inflammation could be caused by flu.

The prevention of smoking and vaccines could be placed in this area and there are studies, which prove the effectiveness of vaccines and anti-smoking activities. However, the goal of this research is to find out what can improve the health management. There is a lack of studies which provide activities of prevention illness of the respiratory system, and it is not practical to implement a question concerning this area.

\section{Prevention of injury at work}

As explained above the German law has detailed information and regulations how accident and injury could be prevented at the workplace. This law is very strict, and the government monitors the injury and work safety. These governmental insurances provide for each branch services concentrated on accident prevention. They do intensive research and very broad activities in the area of accident prevention and every company has access to the support of the BG. They have a focus on different branches and provide services and consultations.

However, the area of work security is a very small problem in medical offices and there is a very intensive monitoring system, that is why the author decided to include no questions for work security in the questionnaire.

\section{Promotion of general health}

The area of general health promotion is broad and as result from the literature review, there are many activities, which could help to improve the health management. Additional there are problems, which are linked with musculoskeletal disorders. For example, overweight can lead to several diseases and to problems in the back, heart, diabetes, and depression, etc.

The promotion of general health has three possible areas which could have been included in the questionnaire:

- Overweight

- Addiction prevention 
- Vaccines

Overweight or obesity is part of the area general health promotion. According to World Health Organisation (Engbers, 2007) workplace programs for improving eating habits are generally accompanied by positive effects, even though they are rather moderate. Nevertheless, they can influence the employee's consumption of fruit, vegetables and fat as well as their intake of dietary fibres significantly as shown in controlled studies. Products and other informational strategies can encourage the sale and consumption of healthier food and thus a healthier food selection of the employees during working hours. Benedict and Arterburn (2008) showed that moderate short-term weight reduction can be achieved. Therefore, the following questions were included in the MFA survey:

Which activities for weight reduction and change of nutrition would you support?

The rating scale for the answers is:

total acceptance tendency to yes neutral tendency to no absolutely no

The answer options for each question is effectively a Likert's scale (1932) and later on the statistical significance will be calculated (cf. 5.2.4).

The question about weight reduction was an ethical dilemma because most participants might not answer honestly about their weight. During the piloting process (Saunders et al., 2012, p.451), a medical physician who cross-checked the questionnaire was afraid that the employees could feel uncomfortable with this question because it assumes that they are overweight. To avoid this problem and the ethical dilemma the author changed the question and added the nutrition program to it. Before it was just "would you join a weight reduction program". This enabled participants who do not have overweight but want to change their nutrition to answer the question.

The prevention of addiction is part of the general health. There are several addictions, which lead to illness and have a negative influence on health. However, the abuse of alcohol or drugs can have a negative impact on the health of the employees. In the literature review, there are several results for programs against addiction. However, there are some studies with a proved influence on the employees' health and the result of reduced absence days. The alcohol prevention is part of several safe- 
ty programs in the work security for industrial workers. The danger of an industrial worker who is working with a machine when he/she is under the influence of alcohol or other legal drugs and non-legal drugs is higher. To prevent accidents is a different perspective on the health management and more important in the non-service sector.

Questions about addiction can lead to an ethical problem. In a small organization is the anonymity for the employees. There are only two or three colleagues and the employer, if an employee takes part in an addiction prevention program this could hardly be anonymous.

However, the goal of the questionnaire is to find out which activities with a proved benefit would be accepted by the employees. The rules against smoking have intensified in recent years. It has been shown that smoking causes several health problems; and that it is not easy to quit smoking. Smoking is prohibited in all offices in Germany and the occupational medicine was one of the main drivers, which promoted prohibition in restaurants, bars, and other event locations. One target of the prohibition was to protect the employees in these locations of passive smoking. There are still different rules in different areas of Germany, and in some regions in private clubs, smoking is still allowed.

Smoking is a danger for health. However, following the research questions, the goal is to find out what can be done to reduce smoking at the workplace, or generally, and does this lead to illness and a better health management. The research from Cahill et al. (2015) analysed several other studies with a focus on smoking prevention and found out which kind of programs work best. Those programs work best against nicotine work, if they are in a group or with a professional face-to-face meeting (Cahill, Hartmann-Boyce et al., 2015). Self-study materials are not as effective. There are no advantages by a competition but by team or group work. This is important information for the design of the questionnaire, because the limited number of questions forced the author to narrow down the important points. Therefore, following Cahill et al. (2015) it is not necessary to ask the participants which kind of program they would prefer, because the teamwork has the best-estimated effect. However, the analysis of the whole area of prediction did not lead to a question, which could help to improve the health management. There would be an ethical dilemma too, because most participants might not answer honestly if they are asked for a drug problem. Especially for MFAs as member of staff of a medical physician it could be a problem if they smoke.

Vaccines are a classic action for preventing disease. To support the general health vaccine can be an important support. Every new-born baby gets basic immunisation through vaccines. Additional and more relevant for the health management are the vaccines which have to be refreshed during the live, special travel vaccines and the yearly flu vaccine. The focus of the research questions is health management in general practitioners' surgeries therefore the main interest of the author is to ask for vaccines, which can have a positive effect on the general health and the absent days. 
This area is strongly regulated through the government and the author is not qualified to pass a judgement on which vaccine is good for working people and who should take it. However, there are several studies, which prove that for example a flu vaccine reduced the average of absent days. All vaccines are proved by the Federal Institute for Drugs and Medical Devices on its effectiveness and then recommended for the daily use by the Standing Vaccination Commission (STIKO). To make the decision which vaccine can be used for effective health promotion at the workplace the recommendations of the German government are used. The vaccines, which are recommended for the employees in general practitioners' surgeries, are included in the questionnaire. The last three questions therefore concern vaccination.

The answer can be "yes" or "no" followed by an open question: "If yes which vaccination? If no, please explain why not:"

The answer can be "yes" or "no" followed by an open question: "If yes which vaccination? If no, please explain why not:"

The answer can be "yes" or "no" followed by an open question: "If no please explain why not:"

As members of a so-called risk group (2.2.6), it must be assumed that the MFAs are adequately concerned with their vaccination protection and have a particularly good vaccination status. It is of particular interest to find out whether a potential improvement potential could be used by a corresponding appropriate offer of the general practitioner.

\subsubsection{Checking the usability of the questionnaire}

After the design of the questions they had to be checked by potential participants. The use of this piloting is recommended, because it aims to avoid a too narrow or subjective focus view by the expert (Saunders, Lewis, \& Thornhill, 2012). By this way, the risk of wrong terminology through the usage of business wording or the medical wording was minimized. The speaking terminus in the medical branch is different from the typical wording in the business area this has been considered in four steps.

1. Rough questionnaire in Word document

The first draft was used to structure all questions in a two-page format.

2. Rough questionnaire checked by author and a medical doctor 
After discussion with a general practitioner and a MFA the first changes were done.

3. Questionnaire in a designed version for physicians

The questionnaire was translated into German and checked by physicians. Further, the feedback was used to refine the questionnaire by highlighting possible overlaps and irrelevant/unclear questions/wording.

4. Questionnaire pilot for employees

The pilot was transferred in a printable design and transferred from word in the survey software. Eight out of 10 pilot participants sent back the questionnaire. There were several ideas for changes and notes.

The "pilot" employees were interviewed after completing the draft questionnaire to find out if the questionnaire is understandable. The aim of this process was to generate a high response rate and to enable all employees to understand and complete the questionnaire. The inhibition level should be as low as possible. The use of piloting is recommended, because it aims to avoid a too narrow or subjective focus view by the expert (Saunders, Lewis et al., 2012). By this way, the risk of wrong terminology through the usage of business wording or the medical wording was minimized.

5. Questionnaire's spelling was checked by a proof-reader

To correct the last spelling mistakes a lector was ordered to proof-read it.

6. Final questionnaire for the research

The final questionnaire was printed and prepared for distribution

\subsubsection{Distribution of questionnaires}

The author works for a company, which organizes medical education events, which are visited by physicians and their employees. The author decided to use the congress infrastructure for the targeted distribution of the questionnaires. The great advantage of this approach was the high return quote; but the author must acknowledge that the data is based on participants at these events/congresses and that this is not the whole of all medical practices.

The selection which congress will be used was made randomly from 320 congresses in the years 2015 and 2016. Different congresses for physicians and their employees have been used to distribute the questionnaires. It was ensured that the questionnaires, since they are addressed to MFAs, were distributed only in events with employees from general practitioners' surgeries. All participants were participant of a medical congresses and every participant got a questionnaire. A total of 400 questionnaires were distributed in this way. The distribution was anonymous; the author 
cannot make a link to the participant. The access control to each congress ensured that the participants are proven medical employees. Each participant must sign an informed consent form.

Following Särndal et al. (2003) a good sampling frame has to meet the following criteria.

1. Numerical identifier

2. All units can be located with complete address

3. The frame is organized in a systematic way

4. Every element of the population of interest is present in the frame

5. There are no doublets within the data base

6. The data is actual

7. The data is in a structured data base

The numerical identifier was created by the OCR-software transferred each answer sheet to a structured database. Since the survey was anonymous, the complete address could not be provided, but the access control ensured that only medical persons have had access. On these congresses, the participants have to register with their full name and the address of their employer. No one can register as a participant without registration, because these congresses are only for physicians and their medical staff. This is necessary because pharmaceutical companies promote their drugs and it is prohibited by law to promote perception drugs to patients. Every participant of a course for medical staff got the questionnaire and the moderator invited the participant to complete it. Afterwards the questionnaires were collected with the additional standard quality questionnaire. In this frame and context, doublets cannot be excluded with $100 \%$ certainty, but the probability is low, since the events took place in different regions and at different times.

The questionnaire results have been transferred to a database for analysis. The main part of the questionnaire consists of quantitative data that has been transferred to a table. Some questions were qualitative. The answers to such open questions have been categorized in groups. These groups were formed so that they were suitable for adoption in quantitative data analysis.

The presentation of the results and the evaluation of the research findings based on the questionnaires are the subject of the following chapter.

\subsubsection{Limitations on convenience sampling}

The MFA survey data collection described above takes advantage of a convenience sample such as ease of use, rapid availability, and cost-effectiveness (Henry, 1990). 
As a working technique of non-random sampling, there are limitations to representativeness (Saunders et al., 2012) that result from the choice (structure), time, place, and content.

The main limitation is the choice of participants. As noted above, while the respondents interviewed are MFAs in German general practitioners' surgeries, the target population and population of this research, there is no information on the proportion of MFAs working to attend training events at all. There is also no information as to which organizers prefer them or their employers. Preferences on primary prevention measures for MFAs who are not willing to train or have no opportunity to attend training sessions are not included in this sampling.

Another limitation arises from the fact that there is no random sample over time. This relationship becomes obvious when one considers that a question about vaccine preferences is answered differently in a current fast-acting flu epidemic (e.g. in the winter months) or a measles outbreak than in the summertime. Although the events in which the questionnaire was distributed were randomly selected, the events themselves are not randomly distributed throughout the year but consider common planning times (e.g. exclusion of holidays and vacation periods).

Also, the distribution and selection over the places was not built by a coincidence principle, which aspired representativeness. This can lead to bias in preferences. Although the events are spread throughout Germany for economic reasons, there are regions where the organizer is under- or overrepresented for historical reasons. In addition, there may be differences in preferences between urban and rural regions. For example, since fitness-centres are much more present in the cities than in rural areas, it can be assumed that there are differences in terms of movement that distort representativeness.

Restrictions resulting from the content occur in both random-sampling and non-random sampling. In terms of content, there are so-called fashion topics on health topics that are sometimes stronger and sometimes weaker. Typical examples can be found on the subject of "healthy nutrition", which is increasingly being brought to the fore by health insurances, women's, sports, fashion magazines and other media, so that the answers to the preference profile may also be influenced by fashion trends.

Despite these limitations, the author assumes that the MFA survey draws an approximately representative picture of the preferences of around 190,000 MFAs in general practitioners' surgeries (BMG: Health system facts, 2016, p. 97), as the target group under educational and professional aspects can be considered largely homogeneous. Following Jacob (2009), a sample of 100-200 individuals may already be representative in specific homogeneous groups with predominantly descriptive purposes. 


\subsubsection{Hypotheses on the results of the MFA survey}

The answers to the individual questions are analysed statistically in terms of their frequency and percentages in the chapter "Research findings of the MFA survey". A further step and a significant contribution to the widening of knowledge about primary prevention in general practitioner practice are the hypotheses presented below, which are accepted or rejected. A hypothesis is an assumption about a certain difference, an effect or an interrelationship. The hypothesis is formulated as a null hypothesis $\left(H_{0}\right)$ and as a counter- or alternative hypothesis $\left(H_{l}\right)$. If the null hypothesis can be rejected based on the hypothesis test, this leads to the assumption of the alternative hypothesis. Therefore, the null hypothesis is usually formulated to assume that there is no significant difference, effect, or context. If this assumption is rejected based on the hypothesis test, the counter-hypothesis necessarily applies. (e.g. Saunders, Lewis et al., 2012)

Example:

The author assumes that the willingness to participate in a health program depends, inter alia, on age. The questions "How old are you?" and the question "Would you join a health improvement activity?" are related. The null hypothesis $\left(H_{0}\right)$ to be tested is accordingly:

$H_{0}$ : The willingness of the MFAs to participate in a health promotion program is independent of age.

This results in the opposite or alternative hypothesis:

$H_{1}$ : The willingness of the MFAs to participate in a health promotion program is not independent of age.

The assumption or rejection of the hypotheses has not only statistical significance. If the counter- or alternative hypothesis $\left(H_{l}\right)$ is confirmed, this means that the willingness to participate in a health program for the elderly should be raised differently than for younger MFAs. If the null hypothesis, i.e. no difference in age, cannot be rejected, there is no need for measures to motivate preventive programs among older employees in a different manner than in younger ones.

In the hypothesis or significance test, the probability of the mistake of rejecting the null hypothesis, though true, is the probability of error, the p-value, or the significance level (Saunders et al., 2012, p. 512). If $\mathrm{p} \leq 5 \%$, one speaks of a significant one, with a value of $p \leq 1 \%$ one speaks of a very significant one and at a value of $p \leq 0.1 \%$ one speaks of a highly significant result. All hypotheses in this research are evaluated at the 5\% significance level. The significance level in this research is determined by the chi square test (Saunders et al., 2012, p. 514). In doing so, the values of the respective answers to the individual questions are compared with the values which would arise if the answers were selected by chance (by dice or arbitrary ticking). If 
the deviation from the expected value (chi square) is too large and is therefore unlikely to be coincidences, it can be assumed that the answers were not generated by chance and the null hypothesis $\left(\mathrm{H}_{0}\right)$ on the given level of significance $(5 \%)$ must be rejected - e.g., $\left(H_{1}\right)$ is true with the probability (1-p), thus with $95 \%$ security (confidence level).

\subsubsection{Age-related hypotheses}

Apart from the possibly age-dependent design of the primary prevention programs for the individual general practitioner's surgery, the aspects of age dependency are of great importance for:

- The drafting of collective agreements for MFAs

- The development of educational programs

- The further development of the setting approach of health insurance companies for general practitioners' surgeries (2.1.1)

In the following, the age-related null hypotheses are formulated, which are tested in the chapter "Research findings of the MFA Survey".

$H_{0}$ : The willingness of the MFAs to participate in a health promotion program is independent of age.

$H_{0}$ : The preferences of the MFAs to participate in one of the programs for the promotion of movement

- Training at the workplace (up to 10 min.)

- Training at home (up to $10 \mathrm{~min}$.)

- Sponsored activity

- Training with step counter or fitness-watch

- Training in a group with colleagues

is independent of age.

$H_{0}$ : The willingness of the MFAs to participate in one of the programs for weight reduction and nutritional change

- Group meeting with external consultant

- Individual talk with external consultant

- One-on-one talk with a physician from the own practice

- Healthy snacks from the employer

is independent of age.

$H_{0}$ : The participation of the MFAs in any of the proposed programs for weight reduction and nutritional change is independent of age.

$H_{0}:$ The regular, up-to-date use of the vaccination offer by the MFAs is independent of age. 
$H_{0}$ : The willingness of the MFAs to be vaccinated by the employer is independent of age.

$H_{0}$ : The regular check of the vaccination status of the MFAs is independent of age.

The aim of the above hypothesis tests is to find out whether age-specific aspects of primary prevention on the MFA survey are justifiable. In this way, the MFA survey can contribute to the national health target of the Federal Ministry of Health, which explicitly demands: "However, in order to be able to implement these general requirements with regard to the elderly and the old population, it is necessary to: Consider how enormously diverse "age" is. Approach points and access routes for more prevention and health promotion must therefore always be applied to the respective target groups and thus to the specific ones. It is therefore a question of an appropriate quality of life, of adequate social integration and of a participation commensurate with the person's condition. " (BMG 2012, p. 13 - translated by author)

\subsubsection{Program-related hypotheses}

These hypotheses are intended to analyse the interrelationships between the three diverse groups of programs. The results enable class allocations and corresponding relevance assignments. For example, the percentage of MFAs that would not participate in any of the offered prevention programs or which have given a particularly high presence to all program groups.

$H_{0}$ : The preferences of the MFAs for the programs for the promotion of movement and for the weight reduction and nutritional advice are independent of each other.

$H_{0}$ : The preferences of the MFAs for the promotion of movement and vaccination programs are independent.

$H_{0}$ : The MFA preferences for the weight reduction / nutritional counselling and vaccination programs are independent.

The two following hypotheses are not intended to be related to the content, but rather to ensure that the responses in the Likert scale (Likert, 1932) scale are not accidental. The check for uniformity excludes the fact that one of the answers was chosen purely randomly. The test for normal distribution excludes the fact that not only the average value was chosen most frequently and the two extremes only relatively rarely.

$H_{0}$ : The preferences of the MFAs for the programs for the promotion of movement are uniformly distributed.

$H_{0}$ : The preferences of the MFAs for the movement promotion programs are normally distributed from one another. 
$H_{0}$ : The preferences of the MFAs for the weight reduction and nutritional counselling programs are uniformly distributed.

$H_{0}$ : The preferences of the MFAs for the weight reduction and nutritional counselling programs are normally distributed from each other.

\subsection{Methods for the development of a priority concept}

The structured literature review generated data on the wider area of health management. This data has been the basis for the questions of the survey, and the design of the questionnaire for the employees. The results from the area of promotion of health and the effectiveness of activities for prevention of disease are used in the questionnaire. These activities had been structured after the medical types of illness and were put into the survey for the employees (Bräunig, Haupt et al., 2015). This structure is used to cover all areas of illness. The International Statistical Classification of Diseases and Related Health Problems the ICD10 standard is a fix framework and it allowed building up a clear ranking. The illness, which leads to the most illness days, has the highest potential to reduce the illness days. This can lead to the paradox situation that a flu vaccine is the most efficient activity for improving the health management and reducing the illness days, but the employees do not want the vaccine. In this case, the best activity is the worst, because one stakeholder can stop it. The same could happen if the employee wants a fitness coach and maybe it is effective for the health management, but the employer is not able or willing to pay for it. Therefore, the perspective of the employer was determined by the measure of costs, the perspective of effectiveness was determined by the research of studies which evidenced the effectiveness and the opinions and wishes of the employees are given by the questionnaires. The employees could score which activity they would prefer, but the selections were reduced on activities, which are effective.

The above example shows the importance of the different perspectives of the stakeholders: the MFAs as affected, the general practitioner as manager of the surgery and the physician as a health professional. In this section, according to the multi-criteria decision-making model, the weighting of these perspectives is presented (green highlighting in the Figure 15). The method for determining the concept of a priority list from the present decision criteria is addressed, too.

A prioritizing concept is, on closer inspection, a ranking problem in which the different perceptions of the stakeholders are placed in a comprehensible order. The decisive factors here are the decision criteria and their weighting. Before the individual decision-making criteria are presented, the MCDA method used here is explained.

\subsubsection{Methods to solve ranking problems - problem structuring}

The multi-criteria decision analysis (MCDA) methods were developed to provide answers to complex questions with several divergent stakeholder interests (Ishizaka 
and Nemery, 2013). The idea of MCDA is not to find the one correct decision, which solves the problem, but the results will be a list with all possible activities ordered by priorities.

This is important for the use in the practical work of general practitioners' surgeries. These organisations have limited resources of time and money to improve their health management. To avoid inefficient activities or activities which are not accepted by their employees, the results of the MCDA are important.

The MCDA provides several different techniques which can be used for the decisionmaking process. Ishizaka and Nemery (2013) provide an overview about these methods. The provided techniques have the advantage that they can be used for a very broad range of decisions and can handle different kinds of data. This advantage can be a disadvantage too. Ishizaka and Nemery (2013) argue that the flexibility and the range of the possible use for these techniques can also be a limitation.

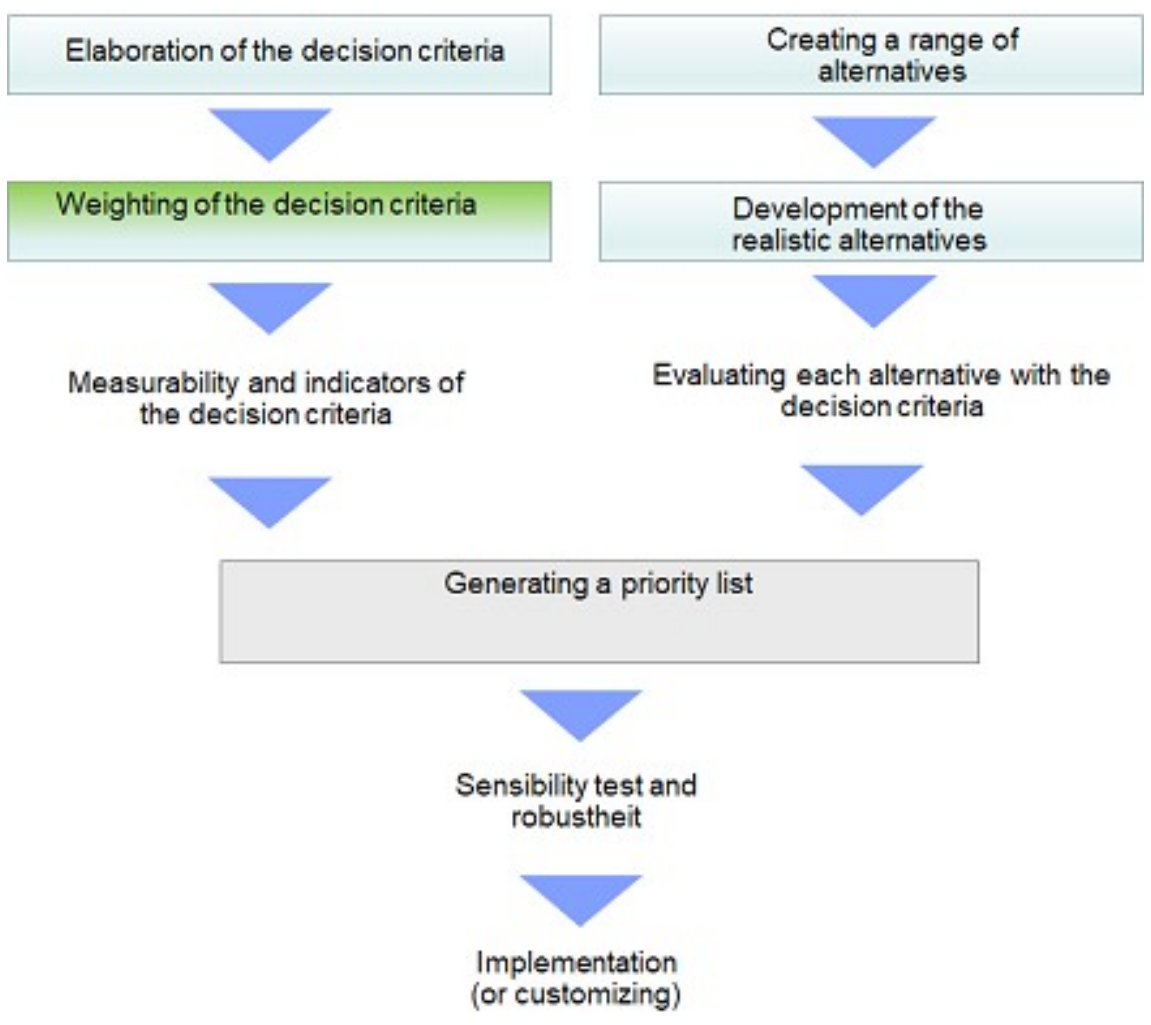

Figure 12: Weighting as part of the multi-criteria decisionmaking model

From the abundance of possible MCDA methods to find the method suitable for a given problem is, on closer inspection, itself a MCDA problem. Ishizaka and Nemery (2013) suggest for the solution of ranking problems to use the selection criteria 
- expected output

- necessary input

- effort input

- possible software support

for choosing the most fitting technique. If, as in the present case, development of a priority list for activities for personal health promotion is expected to be a "complete ranking with scores" as output, and if one expects software support at the same time, four methods will be included in the narrow selection (Ishizaka and Nemery, 2013):

- Multi-attribute utility theory (MAUT)

- Analytic network process (ANP)

- Measuring Attractiveness by a Categorical Based Evaluation technique (MACBETH)

- Analytic hierarchy process (AHP)

MAUT is excluded because the data for all three perspectives needs to have very high quality. A mathematically defined utility function is expected as input, for example, a function which exactly describes how costs affect the benefit (number of days of absence). This could cause problems if the concept is used by the organisations, they may not be able to provide these data.

ANP also for a similar reason should be ruled out, because although no utility function, but the description of dependencies within the decision criteria is necessary. It could be that there is a link between excessive costs for an activity and its preference by employees, which should then be considered in the decision-making process.

Thus the selection shrinks to the two alternatives AHP (Saaty, 1980) or MACBETH (Bana e Costa and Vansnick, 1999). Both techniques have a "complete ranking with scores" as output, need only a pairwise comparison as input, have a "medium" effort for the input and both are supported by a suitable software which can be used for several calculations and weighting. Both are an appropriate choice for analysing quality data. They both have the same advantages as the other multi criteria decisionmaking theories and can be used for complex decisions.

According to Ishizaka and Nemery (2013), the two methods differ with respect to the pairwise comparison. AHP requires a so-called "ratio scale" for the paired comparison, it should be noted, for example, that healthy eating at the workplace is twice as effective as sponsored after-work activities. For MACBETH, on the other hand, the classification is an interval scale, for example "low" or "high". The MCDA method MACBETH with the associated software M-MACBETH is therefore used for the further procedure.

The challenge is to categorize these data and generate useable and valid data for the further use in the MCDA (Ishizaka and Nemery, 2013). The literature research showed that there are many activities, which can be used for improving health man- 
agement. None of the studies provides a suggestion for the one best activity. The result depends on the individual weighting of each element and each activity; therefore, the quality of the result is directly linked to the quality of the provided data and the quality of the scoring. Following the MCDA (Ishizaka and Nemery, 2013) the decision for the activities is built from top to bottom. The thesis and the underlying goal of improvement of health management for general practitioners' surgeries is the main goal. To achieve this goal the criterions needed to be completed and every activity must help to achieve the goal under consideration of the criteria. The goal is the first level, the second level is the criterion and on the third level, there are the alternatives (options).

After the definition of the goal the criteria for the multi-criteria decision analysis has to be defined. The acceptance of the stakeholder is checked by the questionnaire, the effectivity for the activities is based on the literature research and the cost has to be calculated. The goal of the criterion and the following activities is to improve the health management of general practitioners' surgeries.

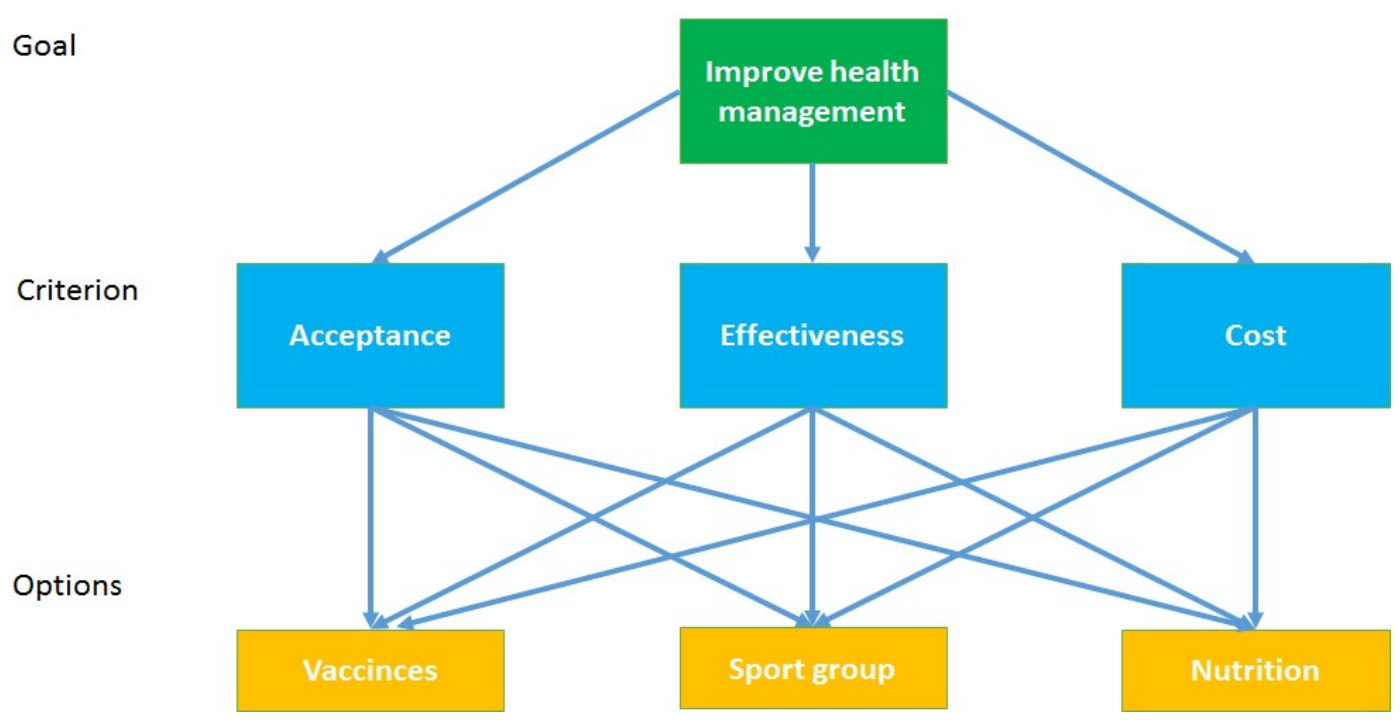

Figure 13: Structuring the problem

The criteria are used as measure to which performance by each option (activity) is achieved. Each criterion and each activity are compared pairwise with each other. Each criterion needs a weighting compared to the other. The acceptance and the data from the questionnaire are the core of this research and therefore the weighting of acceptance compared with efficiency and cost is higher. All three together are weighted with $100 \%$ in sum. The acceptance gets the weighting of $50 \%$ compared to the two other criteria. That is acceptance is for the ranking of priorities "moderate" more important as cost or effectiveness. Effectiveness and costs in turn are weighted equally. There will be three criteria in the analysis. 


$\begin{array}{ll}\text { Acceptance } & 50 \% \\ \text { Effectivity } & 25 \% \\ \text { Costs } & 25 \%\end{array}$

An important aspect in the selection and weighting of the decision criteria is the independence of the criteria. That is, the criteria should not interfere with one another, as otherwise distortions (over-weighting) will occur in the overall assessment.

At level 3 in Figure 13, the options (alternatives) to be ranked and prioritized are listed. For the sake of clarity, they are grouped. The detailed list of all proposed alternatives is shown in the following table. The list summarizes the evaluation of the literature review and includes all essential points of the MFA survey. These alternatives are to be compared in terms of their degree of overall goal achievement.

Since the MFA survey contains open questions as well as the closed answers, the list contains a placeholder for the measures. Further interventions with the general practitioners can also be included. How these additional measures are permanently taken into account is discussed in the research findings. It is important to keep the list open, so that, for example, new research results can be integrated.

\begin{tabular}{|l|}
\hline Programs for activity promotion \\
\hline - Training at the workplace \\
\hline - Training at home \\
\hline - Sponsored activity after work \\
\hline - Training with step counter or watch \\
\hline - Training within a group of colleagues \\
\hline Activities for weight reduction and change of nutrition \\
\hline - Group session nutrition consulting (external) \\
\hline - Single session nutrition consulting (external) \\
\hline - Single session nutrition consulting (internal) \\
\hline - Healthy snacks/food at work \\
\hline Vaccinating programs \\
\hline - Vaccinating programs at work \\
\hline - Vaccinating in private life \\
\hline Further proposed measures \\
\hline - Proposed in the MFA survey \\
\hline - Proposed by the general practitioner \\
\hline
\end{tabular}

Table 3: The list of proposed measures to be prioritized

In the Figure 13, all criteria of level 2 are linked with all options by an arrow. This means that for each alternative it must be decided to what extend the criteria are fulfilled, the so-called scoring. The following subsections show how scoring is done.

\subsubsection{Decision criteria: Acceptance}

The questionnaire gave the primary data for the acceptance. The results of the questionnaires allow to analyse which activities are preferred by the employees. This is core information because the acceptance of employees is needed to implement the 
activities. As an indicator of acceptance, the willingness to participate in a particular activity is used.

The questionnaire gives the primary data for the acceptance. The answers of the questions "Which programs for activity promotion you would join?" and "Which activities for weight reduction and change of nutrition would you support?" are used to define the score of acceptance.

In the five-level Likert (1932) scale (cf. 4.2.3), all answers with "rather yes" and "yes" are counted as agreeing. The answers "neutral", "rather no" and "no" are not considered for the acceptance score. As a score (in points) is added the percentage of agreeing responses. 100 points means that all answers for an activity with "rather yes" or "yes" were marked. A score of 0 points means that no "rather yes" and no "yes" answers were given for this activity.

In the question of the vaccination activities "yes" or "no" or a supplementary text, only the percentage of the "yes" answers is considered, i.e. no response is considered "no".

\begin{tabular}{|l|c|}
\hline \multirow{2}{*}{ Personal health promotion activities } & Acceptance \\
\cline { 2 - 2 } & \multicolumn{1}{|c|}{ Score } \\
\hline Programs for activity promotion & \\
\hline - Training at the workplace & \\
\hline - Training at home & \\
\hline - Sponsored activity after work & \\
\hline - Training with step counter or watch & \\
\hline - Training within a group of colleagues & \\
\hline Activities for weight reduction and change of nutrition & \\
\hline - Group session nutrition consulting (external) & \\
\hline - Single session nutrition consulting (external) & \\
\hline - Single session nutrition consulting (internal) & \\
\hline - Healthy snacks/food at work & \\
\hline Vaccinating programs & \\
\hline - Vaccinating programs at work & \\
\hline - Vaccinating in private life & \\
\hline Further proposed measures & \\
\hline - Proposed in the MFA survey & \multicolumn{2}{|l}{} \\
\hline - Proposed by the general practitioner & \\
\hline
\end{tabular}

Table 4: Scoring the acceptance of personal health promotion activities

Since the responses to potential future actions are targeted, distortions may occur because it is not entirely clear how to respond if one of the measures is already implemented. For scoring, except for vaccinations, it is assumed that no response is given, which indeed lowers the response rate, but does not affect the acceptance rate. If the hypothesis test (4.3.8) shows that the acceptance is different in experienced general practitioners' surgeries, the approach to scoring should be adapted if necessary. The result theoretically could be that all activities are already in use in all gen- 
eral practitioners' surgeries; that would mean that there would be no improvement of health management by this research.

\subsubsection{Decision criteria: Effectivity}

The systematic literature review provided a selection of activities for health management. All activities have a proven effect to the health of employees. This guarantees that every activity, which is provided, has a positive influence on health management. However, this result of the literature review could not be used for the quantitative analyse. There is a need to transfer it into a figure driven score. For the use within the multi-criteria decision-making process each activity will appraised with a factor (score). The scoring is based on figures and the qualitative result of moderate, no or strong effect is transferred into a ratio.

$\begin{array}{ll}\text { None effect } & 0 \\ \text { Low effect } & 25 \\ \text { Moderate effect } & 50 \\ \text { Strong effect } & 75 \\ \text { Very strong effect } & 100\end{array}$

Each activity will be analysed and the studies, which fits, will be associated to the activity. The results of the studies will be categorized and transferred in a figure.

The score is made as follows: The literature research is used to identify the studies for each activity, which show an effect on the disease-related absence. The number of studies multiplied by the factor of the "Level of Influence" then forms the scoring value in points.

For example the „Workplace Physical Activity Interventions: a Systematic Review“ (Dugdill, Brettle et al., 2008) found out there is a moderate influence through activities at work and from the use of step counters. This weighting of his research generates a score of 50 for the training at workplace. The study „How do physical activity, sports, and dietary restraint relate to overweight-associated absenteeism?" (van Strien and Koenders, 2010) found evidence that sports leads to reduced overweight and in their study a reduction of the absence days could be measured. They did not analyse sports during the work time, because of that the proved efficiency for the training at the workplace is weighted as low. The two studies together reach 75 of the possible 200 points or expressed in percentage terms $37.5 \%$ - this value is used in scoring.

The measures to be taken in this research to promote individual health potentials are, ultimately, medical measures of primary prevention. For this area the general practitioner is to be given a certain competence, which is also seen in the self-assessment. Although they do not usually have studies that allow them to better assess the impact on the absence or presence than the literature review, they usually have many years of experience and can assess the effectiveness of the measures related to the physi- 
cian's surgery. This fact can be considered by treating the general practitioner's own experience as one or more studies with a high "Level of influence". Also, own studies can be introduced and evaluated regarding their effects.

The qualitative analysis of the individual studies with respect to each activity and the assessment of their influence level is given in the chapter "Research findings for the development of a priority concept" as well as the average rating of the General practitioners.

\subsubsection{Decision criteria: Costs}

The cost benefit analysis of health management is not easy. There are many studies about the effect of health management activities. However, there are no proved activities, which lead to a profit. Following IGA report (Bräunig, Haupt et al., 2015) in two studies there is some evidence of a general profit from health management activities; i.e. the study provided by the Austrian government (Helmenstein, Kleissner et al., 2004).

To make the decision possible each activity gets a score for its estimated costs. The hours, which will be spend by employees and the money the owner of the medical office must spend - the investment and/or the running annual costs.

The cost factor is fluid. For example, if the general practitioners' surgeries decide to hire a fitness trainer for two hours a week and to provide every employee with a smart watch for measuring their activities. This will cause much higher cost than a fitness coach who has two meetings with the employee, and the employees can practice their learned activities at home. The goal of the cost weighting is to provide an orientation, which is realistic. To achieve this, the costs were primarily measured in the number of internal and external working hours. This has the advantage that the result is relative to the wages of the employees. The same principle was used for the external trainer hours. The costs for a training hour will strongly depend on the region and the kind of trainer who is in charge. Some activities were fully cost neutral, for example, vaccines are paid by the health insurances, and even if the doctors are making the vaccines for their employees, they get money for the work and the vaccine.

Each activity causes cost for the general practitioners' surgeries. To compare these costs, they were categorized for each element.

1. Number of internal employees' hours

2. Number of internal leader (doctoral) hours

3. Number of external trainer hours

4. Estimated external costs in Euro 
The number of internal employees' hours is the time the employees need for the activity and therefore the time they do not make their daily work. If a training group is established during the work time, it cost more time than a training group, which meets after work.

The number of internal leader (doctoral) hours is the time that will be needed by the physician to push the activities. For example, if the physician provides a nutrition course himself because he/she has the knowledge this will cost time.

The number of external trainer hours is used for the estimated hours an external trainer is needed. For example, a fitness coach who visits the office once a month for two hours, or the hours for an external nutrition coach.

Estimated external costs are the cost, which should be planned for buying equipment, for example a fitness watch or healthy food.

As measure working hours were taken and not money per hour, because this allows a better comparison in different regions and with different wages. There are different wages for the same job depending on the region in Germany. To avoid this gap the cost analyses are based on the hours that are needed for each activity. The internal hours are to provide a cost measure that is flexible and keep the activities comparable. To define the real cost of each activity is very difficult because it depends on many factors. A nutrition course can be a course, which takes once four hours and then is finished, it can also mean that a trainer visits the organization every month. To generate results, which could be used in different areas and different organizations, the measure of hours is an option to keep it more comparable. In the sensibility test and robustness check different alternatives can be simulated and discussed.

The cost estimate is based on the author's experience and knowledge of the market and was conducted after consultation with experienced physicians. The cost estimate uses three categories:

High cost or high time consuming

Medium cost or medium time consuming

No cost or not time consuming

100

Measures with a low-cost level therefore receive high points for the score.

A "Cost level" collection sheet is used to record the data for scoring the costs. It is used to define the cost of each activity. It is divided into the three categories above. The columns "cost of working hours" and "external costs" are given the entry "no", "medium" or "high", which is then dotted in the "score" column. "Total score" contains the average of the two "score" columns.

This procedure should establish an estimation of the relative cost level and give an orientation; it does not have the goal to provide hard figures about the activities' cost. For a multi-criteria decision analysis (MCDA) according to Ishizaka \& Nemery, the absolute costs are not decisive. It is important for the decision to know how they re- 
late to each other, and how they relate to each other when compared in pairs. To be able to use the MCDA software, assessments such as "nutritional advice by the physician himself" is more expensive than "nutritional advice by an external consultant". This and other qualitative assessment are done in the chapter 6 "Research findings for the development of a priority concept".

Some activities have clear and transparent costs, because they are paid by the health insurance. A vaccine is completely paid, but the time that is needed to motivate the employee to make the vaccines is not included. In one case, it may be easy and only a reminder for the employee, in other cases this may take a longer time to motivate an employee to take part in a vaccine program.

\subsubsection{Software application: Parameters for M-MACBETH}

To prepare the M-MACBETH software for generating the priority list, the parameters developed in the previous sections were entered.

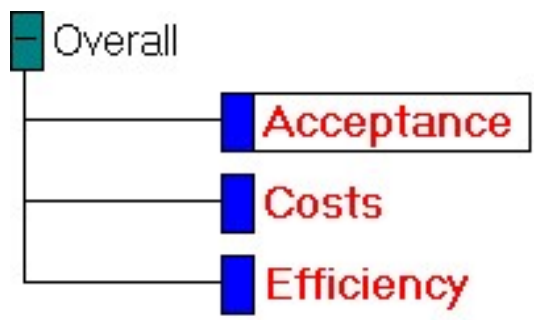

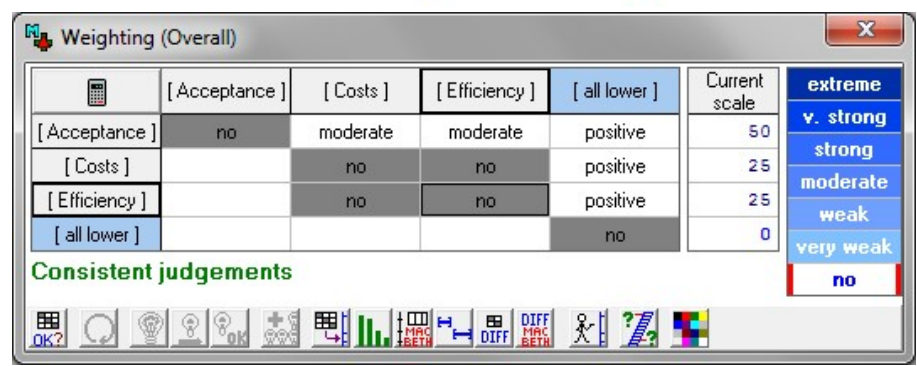

Figure 14: Weighting criteria and consistent judgements (Screenshots M-MACBETH)

When comparing the criteria in pairs, the M-MACBETH software automatically checks whether the comparison data may be contradictory. It would be a contradiction if we were to show in paired comparison that costs are more important than effectiveness, but they are more important than acceptance and that acceptance is the most important criterion. This would be revealed if necessary, in the consistency check. As shown in Figure 14, the weights of the decision criteria are classified as "judgment consistent". 


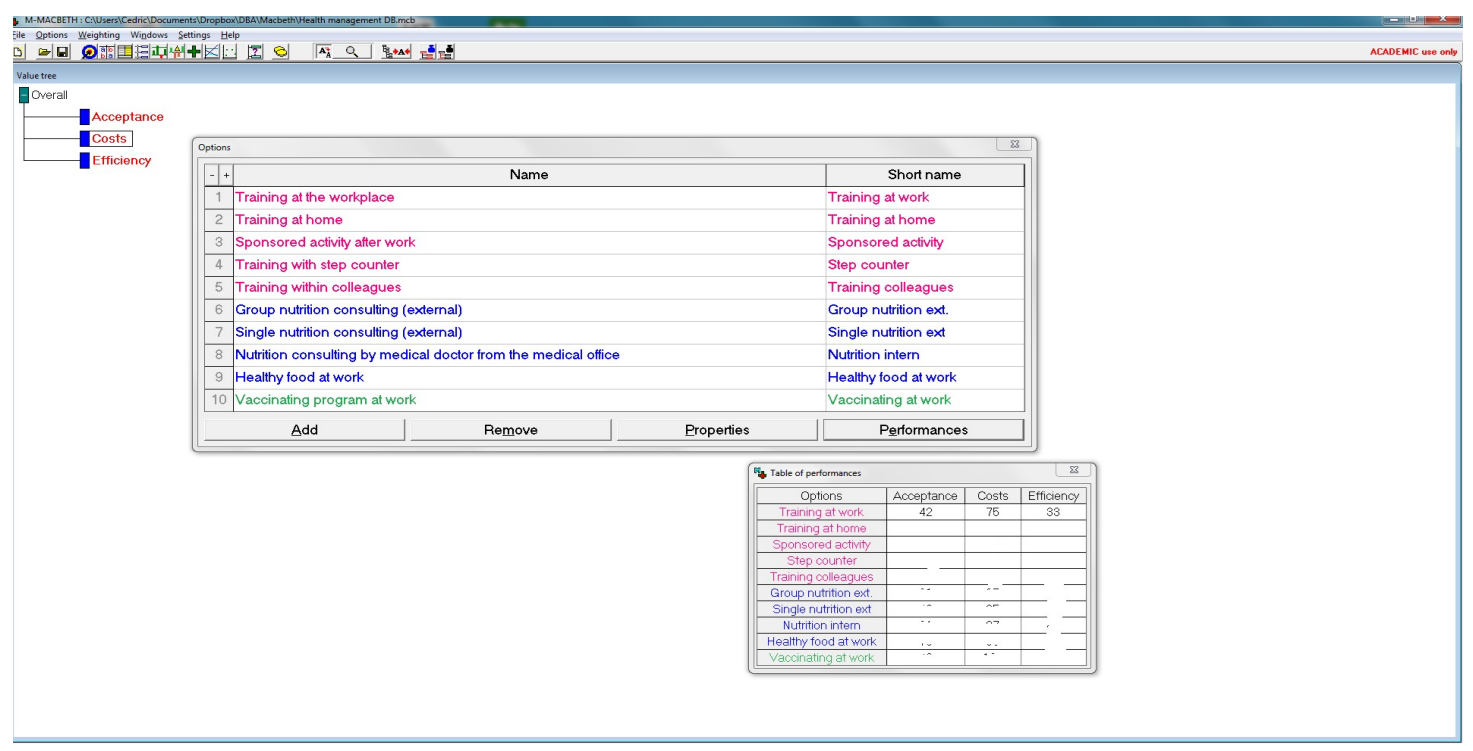

Figure 15: Options and activities (Screenshot M-MACBETH)

To prepare the evaluation all the defined activities have been put into the options list and were linked with the criterion. Distinct colours were selected for the membership of an activity to a particular group.

The final input of the scoring results is given in the chapter "Research findings for the development of a priority concept".

In addition to generating a list of priorities, the software provides powerful possibilities, which could also be used by general practitioners' surgeries. This could be the adding of own activities or the change of the weighting or a portfolio analyse (XYDiagram) and a sensibility analysis combined with a robustness check. These analyses could be done for further research and a better learning about the interaction between the elements.

\subsubsection{Sensitivity test and robustness}

The decision-making process could be completed by generating the priority list. Both methodologically and from the perspective of usage, it is important to assess how the proposed decision-making model behaves if changes are to be made in the weighting or how long the determined priority sequence applies if uncertainties are within a certain tolerance range. Due to the high relevance of such considerations, the sensitivity analysis and the robustness check were introduced as a separate step in the multi-criteria decision-making model, as described in subsection 2.1.5. 


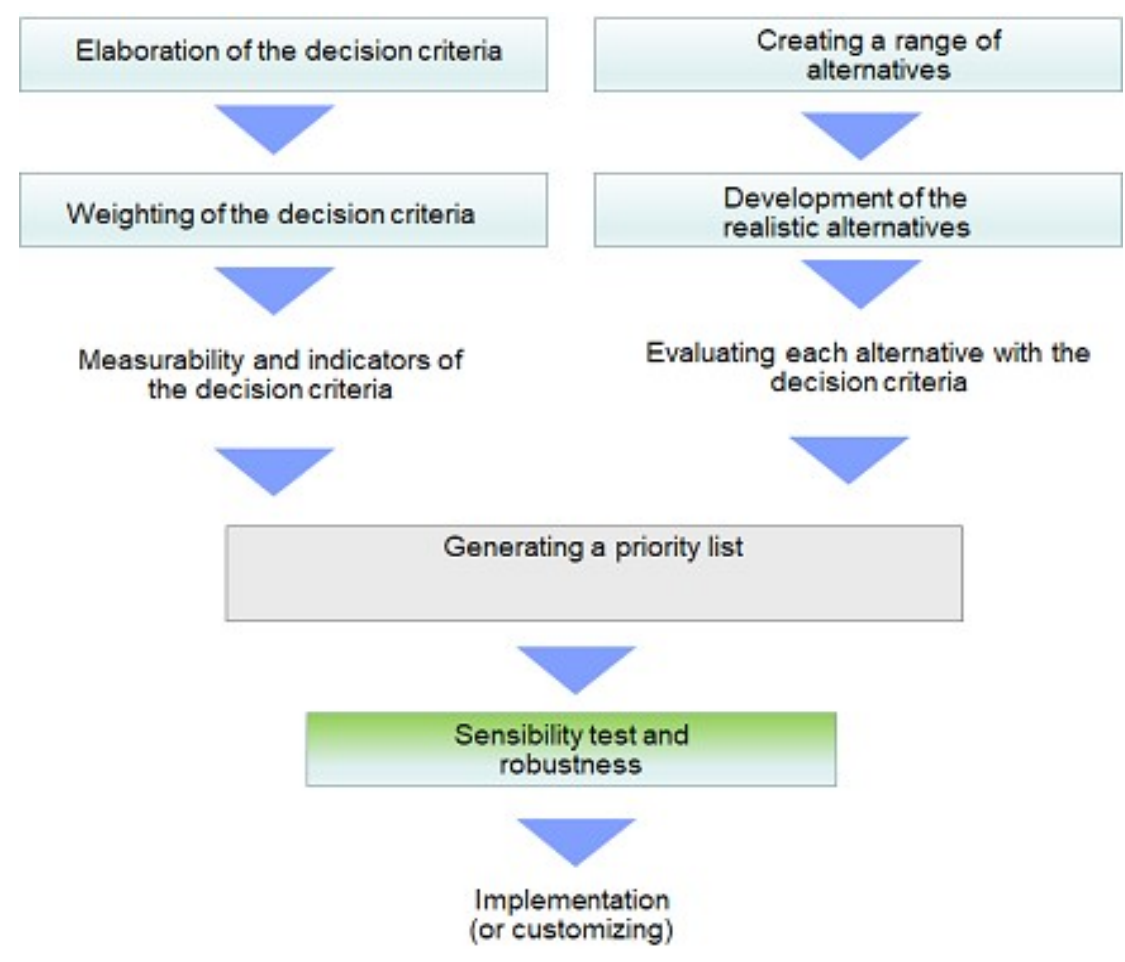

Figure 16: Sensibility test in the multi-criteria decision-making model

Without anticipating the results of the research findings, the following example is intended to illustrate the sensitivity analysis (Figure 17).

The red vertical line shows the current weighting of the decision criterion "costs", which is entered on the $\mathrm{x}$-axis: $25 \%$. The $y$-axis shows the overall rating (considering the two other decision criteria "acceptance" and "effectiveness"). For each selected activity, shown as a blue line, the overall score can be read from the intersection of the red and blue lines. For example, the total scoring of "Healthy food at work" is $68 \%$ of the possible 100 points. The "Nutrition intern" (internal nutritional consulting by the doctor) is less favourable than the individual advice with an external nutrition consultant.

For a general practitioner's surgery, which is economically not so well placed, it might be useful to assign a higher weight to the costs, for example to $40 \%$. Certainly, it would be valuable to know for this surgery that, as a result of this weighting, internal nutritional counselling would perform better than both forms of external nutritional advice. This can be recognized by the dotted red line and the intersection point of the blue lines of "Nutrition intern" and "Single nutrition ext.". It can also be seen that, even with even greater weighting of the "Costs" within the food-related activities, "Nutrition intern" always remains secondary. 


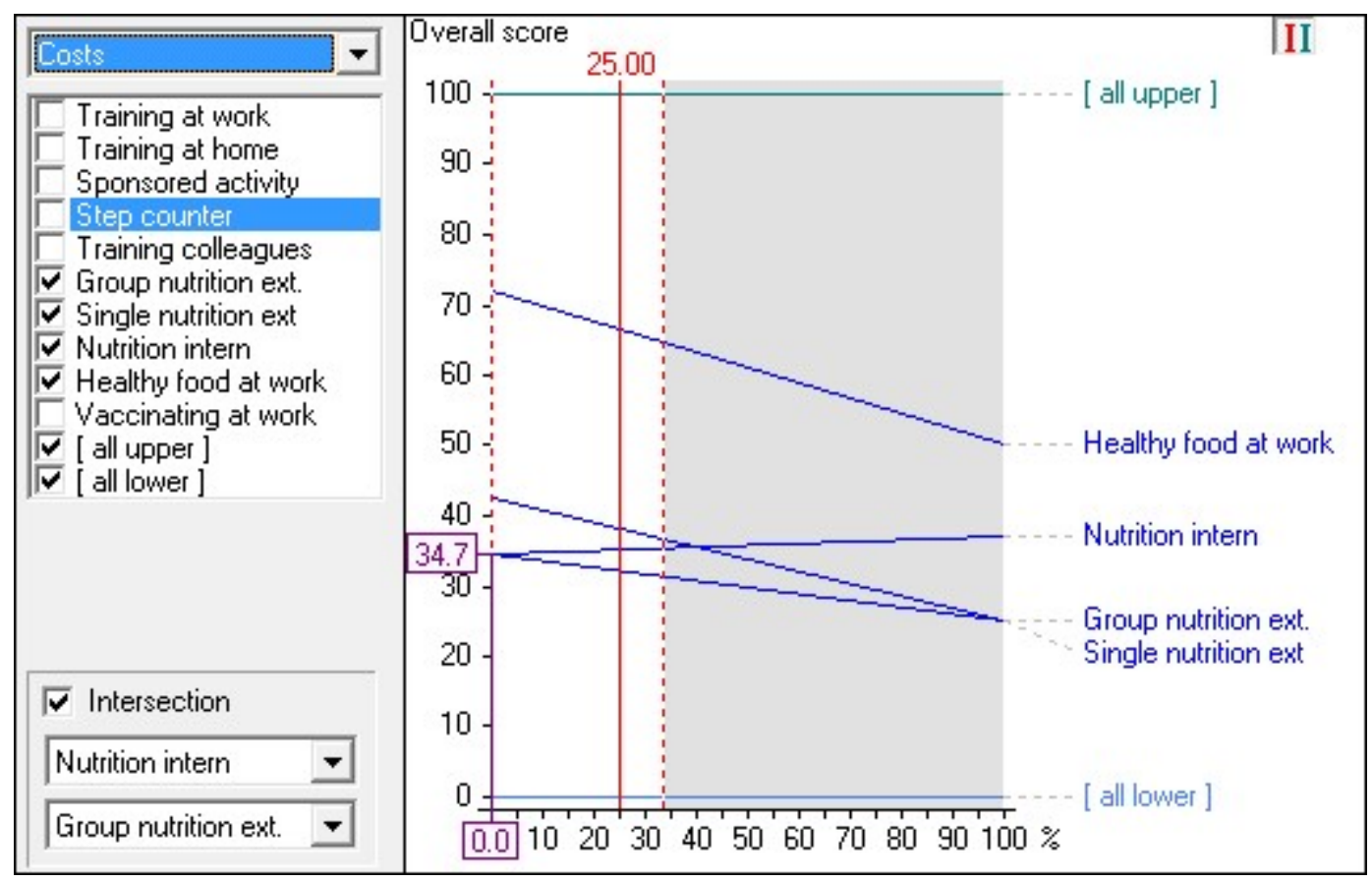

Figure 17: Example of a sensibility analysis (Screenshot M-MACBETH)

\subsubsection{Hypotheses}

The statistical evaluation of the MFA questionnaires led to the hypotheses presented in subsection 4.2.7. The extension of the evaluation by the criteria of cost and effectiveness allows hypotheses to be made about the relationships between acceptance and the individual measures or groups of measures. In addition to the general contribution to the widening of knowledge about primary prevention in general practitioner practice, the significance test for the preference of effective measures provides an initial indication as to whether it is realistic that delegation tasks, as proposed in subsection 3.2.2 Figure 9 should be adopted.

In detail, the following hypotheses are tested in the chapter "Research findings for the development of a priority concept":

$H_{0}$ : MFAs favour the costlier of the individual programs for the promotion of health (i.e., the two features are correlated strong).

$H_{0}$ : Cost level and effectivity of the individual programs for health promotion are not independent (i.e., the two features are correlated strong).

$H_{0}$ : MFAs prefer the more effective of the individual programs for the promotion of health (i.e., the two features are correlated strong).

Another reason for these hypothesis tests is the independence of decision-making criteria. If the decision criteria correlate with one another, changes in the weighting should be discussed. 


\subsubsection{Methods for evaluation of implementation aspects}

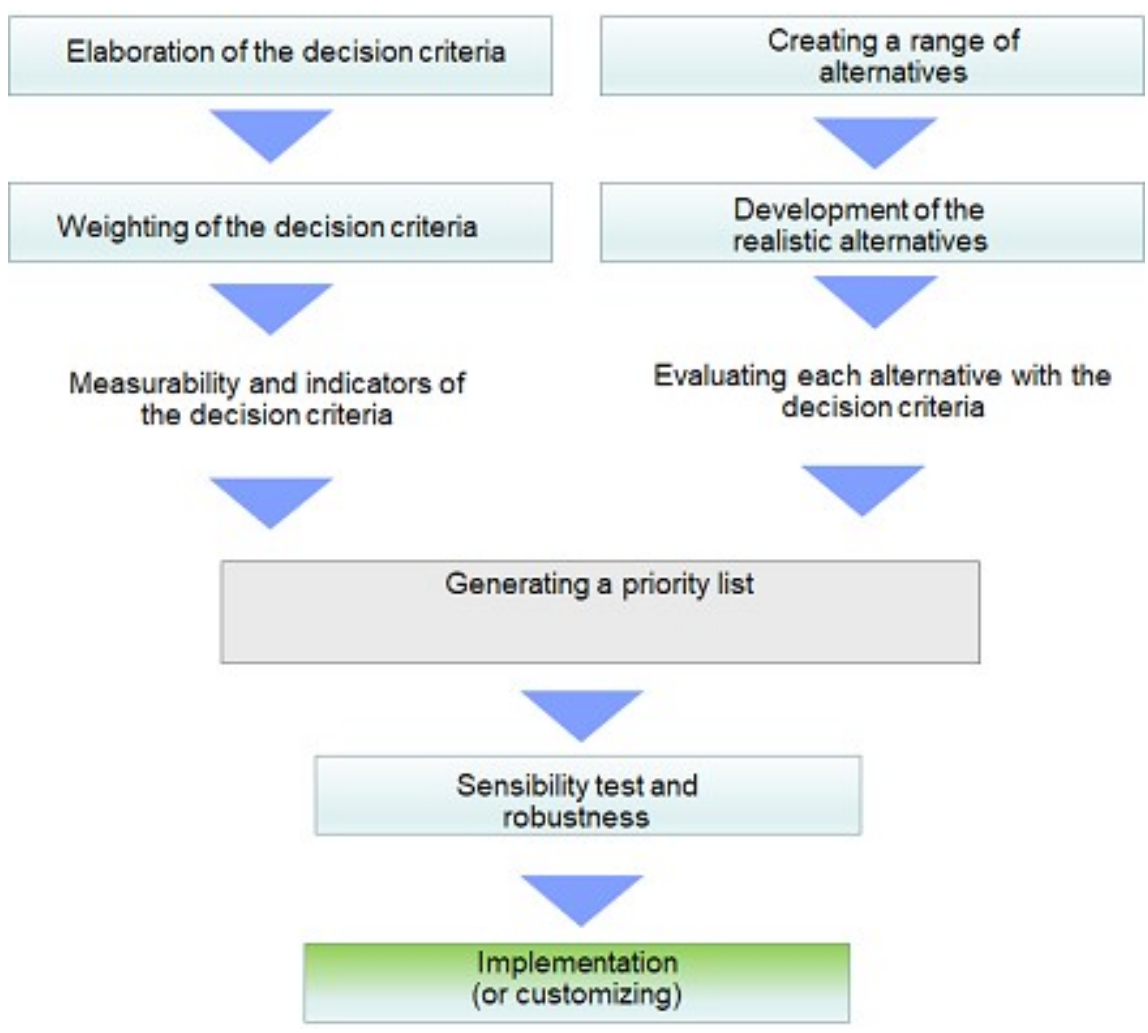

Figure 18: Implementation - the last step in the multi-criteria decision-making model

The last step in the multi-criteria decision-making model is the implementation or adaptation to user-specific needs (Figure 18). Finally, the general practitioner must decide whether to integrate the proposed measures into his/her practice organization. To clarify this was the subject of qualitative interviews.

To reflect the results of the MFA survey, four structured interviews were conducted in each one lasting one hour. In brackets the intended talk time. Before the call or meeting, the doctors were given a sheet showing the list of results with the priorities, see Appendix 5, as well as a copy of the cause-effect network with the health management target system. As the interviews were announced in advance, it was to be assumed that the doctors had informed themselves as far as possible. The following paragraphs outline the topics and the planned time of the interviews:

Classification and characteristics of one's own practice (5 min.)

The size (number of MFAs and age structure), specific features and offers, the existing quality management system and, if available, the health management system and its anchoring, if necessary individual measures of the practice with health care were also recorded. 
Comparison of the priorities list with own ideas (15 min.)

Different assessments were made of the priorities list in the weighting and the ranking of the measures in terms of acceptance, costs and effectiveness. In the case of serious deviations, the readiness was queried to use a software (e.g. Excel), if necessary, to be able to carry out user-specific inputs. Furthermore, the possible time saving using the standardized priority list as compared to own development was requested.

Factors influencing productivity and performance (20 min.)

Based on the cause-effect network, the interrelationships were discussed, and a subjective assessment of the control lever was asked: primary prevention - psychosocial well-being - work organization - job design - own leadership quality. As to the cause-effect network itself, it was asked whether there were missed any essential relationships or control levers.

Implementation of health management in one's own practice (20 min.)

After clarifying the importance of health management in their own practice, a possible sequence of the next steps was discussed. The introduction concept was discussed as to whether health management is seen as an integral part of quality management or whether it is an independent, permanently installed initiative or as a sum of individual and situational measures that are deemed to be reasonable and necessary. Concluding, concepts of "healthy leadership", "health culture in the medical practice" and "health competence MFA" were addressed.

The results of the interviews are found in the chapter "Discussions".

\subsection{Ethical considerations}

Health is a very personal matter. Therefore, the ethics has been very important in this research. To avoid problems the ethical rules provided by the University of Gloucestershire have been strictly followed. The following subsections describe this background with a direct reference to the questionnaire.

\subsubsection{Data protection and protection of the participants}

Following the data protection guidelines of the University of Gloucestershire the primary responsibility for the conduct of ethical research lies with the researcher. Where applicable, professional codes of conduct of external organizations take precedence over the University's expectations and requirements for the conduct of research, although in most cases final approval of research projects remains with the record of ethical conduct (REC). As there was no direct contact with potentially vulnerable individuals, the research did not require REC approval. 
Therefore, the researcher will be responsible towards research participants (including themselves): to ensure as far as possible that their physical, social and psychological well-being is not detrimentally affected. Towards other researchers he will avoid, wherever possible, actions which may have deleterious consequences for other researchers, or which might undermine the reputation of their discipline.

1. Research will be based, as far as possible and practicable, on the freely given informed consent of those under study. The researcher will:

- explain to participants the aims, nature, conduct, funding, duration, purpose and consequences of research, and how results will be disseminated;

- give due consideration to the power imbalance between researcher and researched, and the right of participants to refuse participation at any time;

○ explain to participants the extent to which they will be afforded anonymity and confidentiality, and their option to reject data-gathering devices such as audio recorders etc.

- discuss potential uses of data with participants and obtain their agreement;

- give due consideration to the interests of any 'gatekeepers' where access is gained via a 'gatekeeper';

o where research participants are young children or other vulnerable groups, consult relevant professionals, parents/guardians and relatives, and attempt to obtain informed consent of participants, their parents and those who are in loco parentis;

$\circ$ anticipate and guard against any possible harmful consequences of research for participants.

2. The researcher will not use deceptive methods. The anonymity and privacy of participants will be respected, and personal information will be kept confidential and secure. The researcher complies with the provision of the Data Protection Act 1998 (UK). While taking every practicable measure to ensure confidentiality and anonymity, they will also take care not to give unrealistic assurances or guarantees.

3. There will not be a requirement for specific approval from the Research Ethics Committee.

4. Additional to the rules of the University of Gloucestershire the rules of the German law about data protection (BDSG, 2017) will be respected. 


\subsubsection{Anonymity and possible ethical conflicts}

Health is a very personal and sensible area. All information, which is generated by the author, are anonymous at any time, the questionnaire cannot be associated with one individual person. The questionnaire was given to the participants at an event and collected after the event. The author could access the database, which includes all participants of the event, but it is not documented who of the participants completed a questionnaire. There is no possibility to find out who completed it.

The questionnaire is not asking for the personal health status to avoid possible ethical dilemmas. A question, which could have had the potential for an ethical conflict, is the question for a flu vaccine, because an employee in a general practitioner's surgery could infect ill or elderly people with the flu. However, in Germany the flu vaccine is not obligated for healthcare workers. This possible conflict is a political decision; there was no ethical conflict for the researcher. This means if an employee in a doctor's office and has not been vaccinated, they could be a danger to the patients during the incubation time. The German health office is responsible for such conflicts and the law decides if there are forced vaccines.

\subsubsection{Role of the researcher and ethical considerations}

Following Saunders et al. (2012, p. 236), the ethical issues relating to "right to quality research" and "maintenance of objectivity" are of particular importance during the phases of a research process. Qualitative deficiencies jeopardize the reliability, credibility and transferability of the results. Without objectivity the analysis and the evaluation of the data are impaired, in particular the reproductivity of the results relevant for all research work is endangered.

In the case of the qualified interviews, the ethical consideration is that in addition to the above-mentioned guarantee of anonymity and confidentiality, the independence of the interviewees must be guaranteed. None of the interviewees should be aware of the answers of others in order to avoid possible distortions of third-party comments.

Essentially, the quality of research, as well as objectivity, is affected by two potentials for bias. On the researcher's side, the so-called confirmation bias is the main factor. On the participant's side, it is the social desirability that can falsify results. Both are discussed below with reference to the thesis.

Regarding ethical considerations, it should be emphasized that activities with a concrete reference to personal health issues (e.g. smoking, drinking habits) have been deliberately excluded. This avoided both a possible confirmation bias (attitude and attitude of the author to addictive behaviour) as well as the expected answers with socially desirable content.

\subsubsection{Confirmation bias}


"Confirmation bias means that information is searched for, interpreted and remembered in such a way that it systematically impedes the possibility that the hypotheses could be rejected - that is, it fosters the immunity of the hypothesis. Here the issue is not the use of deceptive strategies to fake data, but forms of information processing that take place unintentionally." (Oswald and Grosjean, 2004, p.79). The term goes back to the cognitive psychologist Wason (1960), who pointed out that we tend to choose and interpret our information in a way that confirms our own expectations. Saunders et al. (2012, p.192) see this as one of the main threats to the reliability of the research work. For if the prejudices of a researcher leading to the confirmation bias have a lasting effect on the result, the repeatability of the examinations with the same or at least similar results by other researchers can no longer be guaranteed.

Before starting to suggest approaches that may involve confirmation bias in this work, it should not go unmentioned that in modern science theory the assumption of existence is considered a confirmation bias itself as one of the most serious confirmation biases. For example, Whittlestone (2017) states in his thesis "The importance of making assumptions: why confirmation is not necessarily a bias": "When we define confirmation bias more precisely, it becomes evident that much of the research commonly cited does not show what it claims to. Most (if not all) of the evidence we discuss against a conceptual bias in the sense of a systematic deviation. This is often because it is not clear what are the relevant normative standards for the task, the tasks are too specific to generalize to a 'systematic' tendency, and / or because it's unclear focal hypothesis. In addition, almost all research has been done at a bias at the stages of reasoning independently - but we need to understand all stages of the reasoning process, to the point of actually updating beliefs, if we want to be able to conclude that reasoning actually systematically favors the focal hypothesis." (Whittlestone, 2017, p. 193)

A first starting point in self-reflection to uncover possible confirmation biases lies in the selection process of the activities for personal health promotion to be investigated. According to which criteria are certain activities accepted as suggestions and others not? Here, the chosen selection process is of crucial importance. Restricting the inclusion of only those measures that have been scientifically proven to reduce absent time will preclude actions based solely on the author's personal experience and judgment, as well as those of marketing interests of product manufacturers (e.g. dietary supplements) and service providers (e.g. gyms). A typical example is yoga, which according to the author's experience belongs to the health-promoting personal measures, for which no scientific proof was found that it reduces the absence rates.

By this selected selection process, a possible problem with confirmations biases is shifted from the author to the researchers of the respective studies or to reliability of the institutional level of the publisher.

A disadvantage of the chosen selection process, so to speak a confirmation bias of the second kind, is the filter of "scientificity". As a rule, only activities were included 
that were marked as peer reviewed by academic journals. Thus, it cannot be ruled out that there are measures that certainly help in everyday practice but have failed to appear in an academic journal and to be peer reviewed.

To minimize the risk of confirmation biases regarding possible activities, an open question was included in the MFA survey with room for own suggestions and in the structured interviews with the general practitioners, possible further measures were asked.

The second starting point for a possible confirmation bias lies in the multi-criteria decision-making model. Here it can be argued that the author's prejudices led to a one-sided selection of decision-making criteria. Thus, it cannot be denied that the author attaches significant importance to the acceptance of measures by the employees and that this becomes more or less obvious when recommending a priority concept. However, this is transparent in the decision-making process and the chosen approach of consciously considering the diversity of perspectives (acceptance/employee - effectiveness/doctor - costs/owner) was not least chosen to rule out unilateral confirmation bias.

Another measure to counteract a confirmation bias lies in the integration of the proposed measures into a comprehensive cause-and-effect structure and in a proven process model.

According to Whittlestone's thesis (2017), this study is based on two assumptions: first, the assumption that health management measures can contribute to improved performance in general practitioners' surgeries, and second, that decision-making on the choice of measures follows a rationality-driven decision-making process.

\subsubsection{Social desirability}

"Social desirability bias refers to the tendency of research subjects to give socially desirable responses instead of choosing responses that are reflective of their true feelings. The bias in responses due to this personality trait becomes a major issue when the scope of the study involves socially sensitive issues such as politics, religion, and environment, or personal issues such as drug use, cheating, and smoking." (Grimm, 2010 , p. Social desirability) the confirmation bias, socially desirable answers endanger the reliability and validity of the research results.

Certainly, the topic of "health" is associated with socially desirable answers. It is tacitly assumed that "health and wellbeing" should be regarded as desirable conditions, and that activities aimed at doing so should definitely be endorsed. This is especially true for health professionals, such as the MFAs surveyed here. They are believed to be open-minded to health management of any kind.

This relationship may lead to a participant bias (Saunders et al., 2012, p.192) when assessing the acceptance of individual measures as the popularity of measures may tend to be overstated. This would be important if only one measure were to be as- 
sessed. However, since the preference of several measures is to be assessed in comparison, it can be assumed that none of the individual measures turns out to be particularly socially desirable.

As Grimm (2010) suggests, one of the ways to deal with social desirability is to provide well-elaborated response options to those questions that are prone to the social desirability effect. This proposal was followed in questioning the specific activities. In addition, it is not clear to what extent a truthful answer (instead of the socially desirable ones) could lead to disadvantages or rejection. The chosen form of anonymization does not allow any conclusions to be drawn about an individual person.

In the individual measure "vaccination" a social desirability can conceptually not be excluded. One focus of public vaccination campaigns aimed at counteracting increasing vaccine drowsiness is so-called herd immunity. This refers to the effect that immunity acquired through vaccination is so widespread that unvaccinated individuals are also protected in the population (the "herd") because the pathogen cannot spread. Here consciously a "socially desirable" behaviour is propagated, as it also protects immunocompromised persons (older, newly born, sick, ...) who cannot be vaccinated. From the answers in the questionnaire it is not clear to what extent the advocacy of the prevention measure corresponds to vaccination of a personal conviction or a desired behaviour.

When the general practitioner uses the multi-criteria decision-making model, it cannot be ruled out that socially desirable behaviour plays a role. So, the general practitioner is certainly aware that the employee orientation today is given an increasingly high priority. This tends to increase the emphasis on the acceptance of healthpromoting measures. It is not possible to clarify based on interviews whether the lip service in favour of the employees was filed in the general practitioners' interviews or whether this corresponds to the actual conviction and the practiced action.

From an ethical point of view, the multi-criteria decision-making model should be evaluated from the perspective of "maintenance of objectivity". From the author's point of view, transparency and traceability in the derivation and weighting of the decision criteria is an essential contribution to the "maintenance of objectivity".

The situation is similar with the statutory duty to quality management. To what extent this is the simple fulfilment of a socially set framework conditions, more precisely and strictly formulated a social enforced behaviour, or a lived conviction in the patient's interest cannot be clarified by the interviews.

\subsection{Summary}

The aim of the chapter was to discuss and elaborate the methodological foundations for answering the research questions. At first, the research paradigm was contrasted in the dimensions "nature of science" and "nature of society". From the resulting possibilities "radical humanist", "radical structuralist", "interpretivist" and "functional- 
ist", the latter was identified as the most adequate for this research work and at the same time closest to personal attitudes.

The four common research philosophies positivism, realism, interpretivism and pragmatism were presented and underpinned by references to health management. The research philosophy underlying this research has been identified as a pragmatic approach based on a conceptual framework that combines HR practices, psychological contract, and organizational performance. In this framework, each of the five research questions can be anchored.

The axiological point of view of the research philosophies was a pragmatism one. The focus is therefore to identify and minimize the effects of biases.

In the ethical considerations, the data protection rules accepted as binding and the guidelines for the protection of the participants were explained and explicated. Likewise, anonymity and possible ethical conflicts were discussed. According to the axiological standpoint of a pragmatism, a possible confirmation bias and possible socially desirable answers were discussed in the related issues.

The methodological basis for the research philosophies is an explorative and explanatory approach, which is augmented by questionnaires and structured interviews in a two-stage research design. Accordingly, the methods for developing a questionnaire for acceptance by MFAs and a priority concept were presented. As far as possible, hypotheses have been prepared for both areas, the validity of which should be reviewed later.

While this chapter describes the methods used to design the MFA survey and to develop the priority concept, the next two chapters will focus on the application of the methods and the research findings to be derived from them. 


\section{Research findings of the MFA survey}

Section 1 of this chapter presents all recorded data in absolute and percentage values. Attention was paid to assessing qualitative data and assessing them in the context. The aim of section 2 is to draw a clear picture of the preferences of MFAs based on the results of the survey. Links e.g. between individual measures and their acceptance in different age groups are clarified. As a contribution to knowledge about health management in general practitioners' surgeries, the statements regarding their statistical significance are examined. This provides a basis for rejecting or retaining the hypotheses (4.2.7). In section 3 the results are summarized.

\subsection{Quantitative and qualitative data from the MFA survey}

The questionnaire was printed 400 times. It was distributed on several medical congresses with assistance personal as participants in the period September 2015 to March 2016. The feedback from the participants was very positive. The response was higher as estimated and over $50 \%$ of the distributed questionnaires have been completed. Every second participant completed the questionnaire. When the questionnaire was distributed, the moderator asked or invited all participants in the auditorium to kindly complete the questionnaire. This may have led to a relatively high response quote. Originally, certain software should collect the completed questionnaires. This did not work because the open question could not be processed by it. However, the questionnaires were collected and evaluated in an Excel table (Appendix 4). Questionnaires which were completed less than $80 \%$ were excluded - this was the case for only ten of the questionnaires.

The following table shows how many questionnaires were distributed. It includes the absolute number of returned questionnaires and the percentage in relation to the total number of distributed questionnaires.

\begin{tabular}{|l|l|c|}
\hline Status & Number & Response rate (\%) \\
\hline Distributed questionnaires & 400 & $100 \%$ \\
\hline Returned questionnaires & 204 & $51 \%$ \\
\hline Analysed questionnaires & 194 & $48.5 \%$ \\
\hline
\end{tabular}

Table 5: Number of questionnaires with return rate

\subsubsection{Data on demographic characteristics}

Two questions provide information about the participants: the age and the gender. The data in this first question is quantitative, there were no open questions.

\begin{tabular}{|l|r|r|}
\hline Gender & Total & Percentage \\
\hline Female & 194 & $100 \%$ \\
\hline Male & 0 & $0 \%$ \\
\hline
\end{tabular}

Table 6: Gender of the MFAs 
The result for gender is a $100 \%$ female. No male completed the questionnaire. To avoid a mistake, the lists with the participants of the congresses where the questionnaire was distributed were checked really there were no male participants.

The result for the age of the employees is useful to learn about the actual and potential future problems linked with aging employees and the result is also useful to recognize age-related preferences.

\begin{tabular}{|l|r|r|r|}
\hline Age & Total & \multicolumn{1}{|c|}{ P1 } & P2 \\
\hline $18-25$ & 59 & $31 \%$ & $30 \%$ \\
\hline $26-35$ & 34 & $18 \%$ & $18 \%$ \\
\hline $36-45$ & 49 & $26 \%$ & $25 \%$ \\
\hline $46-55$ & 35 & $18 \%$ & $18 \%$ \\
\hline $56-65$ & 15 & $8 \%$ & $8 \%$ \\
\hline $66+$ & 0 & & \\
\hline Total answer & 192 & & \\
\hline No answer & 2 & & $1 \%$ \\
\hline Total questionnaires & 194 & & \\
\hline
\end{tabular}

Table 7: Age of the MFAs

In the P1 column the percentage is calculated related to the number of total ticks and in $\mathrm{P} 2$ the percentage related to the total number of questionnaires. These two figures are calculated to show how many participants completed the single question.

\subsubsection{Data on the general practitioner's surgery}

In the subsection 4.2.2 the selection of general practitioners' surgeries has been defined. These are the results of the control questions to be sure the right group of employees has been targeted.

The questionnaires were distributed on congresses for general practitioners and their employees. The question for the speciality is to make sure the group of employees which was selected is correct. During the congress registration, the participants had to confirm that they are working in a medical doctor's office, but the speciality was not interrogated.

\begin{tabular}{|l|r|r|r|}
\hline \multicolumn{5}{|l|}{ What kind of general practitioner's surgery you work for? } \\
\hline & Total & \multicolumn{1}{c|}{ P1 } & \multicolumn{1}{c|}{ P2 } \\
\hline General practitioner & 165 & $92 \%$ & $85 \%$ \\
\hline Gynaecologist & 9 & $5 \%$ & $5 \%$ \\
\hline Other & 6 & $3 \%$ & $3 \%$ \\
\hline Total answer & 180 & & \\
\hline & & & \\
\hline No answer & 14 & & $7 \%$ \\
\hline Total questionnaires & 194 & & \\
\hline
\end{tabular}

Table 8: Specialization of the doctor's office, in which the MFAs work 
In the P1 column the percentage is calculated related to the number of total ticks and in $\mathrm{P} 2$ the percentage is calculated related to the total number of questionnaires.

The quantitative data on the existing health management programs were generated by a multiple-choice question. There was no further specification of the kind of activities. The participants could interpret health management from their point of view.

\begin{tabular}{|l|r|r|r|}
\hline $\begin{array}{l}\text { Are there activities for health management in your general practi- } \\
\text { tioner's surgery? }\end{array}$ & Total & P1 & \multicolumn{1}{c|}{ P2 } \\
\hline & 45 & $26 \%$ & $23 \%$ \\
\hline Yes & 129 & $74 \%$ & $66 \%$ \\
\hline No & 174 & & \\
\hline Total & & & \\
\hline & 20 & & $11 \%$ \\
\hline No answer & 194 & & \\
\hline Total questionnaires &
\end{tabular}

Table 9: Existing health management

In the P1 column the percentage is calculated related to the number of total ticks, and in $\mathrm{P} 2$ the percentage is calculated related to the total number of questionnaires.

Additional there are answers to the open questions which provide qualitative data. There were in total 71 comments. Multiple answers were possible. For categorizing, the open question was divided in two parts "yes" and "no". In the following table, the grouped answers for the questions are summarized. The spellings of the suggested activities were different but clear enough for categorizing them. The proposed activities were only difficult to read, but sufficient to categorize them.

\begin{tabular}{|l|r|r|}
\hline \multicolumn{3}{|c|}{$\begin{array}{c}\text { Are there activities for health management in your general practi- } \\
\text { tioner's surgery? If "yes" what kind of activity? }\end{array}$} \\
\hline & Total & Percentage \\
\hline Check up & 12 & $30 \%$ \\
\hline Vaccine & 14 & $35 \%$ \\
\hline Nutrition advice & 5 & $12.5 \%$ \\
\hline Healthy food & 3 & $7.5 \%$ \\
\hline Office chair for the back & 1 & $2.5 \%$ \\
\hline Fitness & 3 & $7.5 \%$ \\
\hline Vitamin infusion & 2 & $2 \%$ \\
\hline Total & 40 & \\
\hline
\end{tabular}

Table 10: Existing health management activities for the open question "yes"

The following question should only be completed if the answer to the question above was "no". The results from the qualitative answers were categorized in groups. The following table above summarizes these answers. 


\begin{tabular}{|l|r|r|}
\hline $\begin{array}{l}\text { Are there activities for health management in your general practitioner } \\
\text { surgery? If "no", why are there no health management activities? }\end{array}$ \\
\hline & Total & Percentage \\
\hline I do not know & 9 & $32 \%$ \\
\hline No interest / nobody care & 11 & $39 \%$ \\
\hline No time & 8 & $29 \%$ \\
\hline Total & 28 & \\
\hline
\end{tabular}

Table 11: Possible reasons for lack of health management for open question "no"

In the next table, the group who answered "No - there is no health management in the organisation I work for" is summarized.

Five answers in both parts of the question were not included, because they were not linked to the question like "I work too much and earn too little" or they were illegible or not understandable.

\subsubsection{Data on the promotion of personal health potentials}

The first question was the willingness to participate in health management and its general acceptance. This was a question with a multiple-choice option and an open question. The question if a MFA would generally join a health prevention program was divided in a quantitative and a qualitative question. The participants could answer these questions independently of an existing health management program in their general practitioners' surgeries.

The quantitative part of the question was designed as multiple-choice question. It was combined with an open question afterwards. The following table shows the result of the quantitative data.

\begin{tabular}{|l|r|r|r|}
\hline \multicolumn{4}{|l|}{ Would you join a health prevention program? } \\
\hline & & P1 & P2 \\
\hline Yes & 97 & $68 \%$ & $50 \%$ \\
\hline No & 46 & $32 \%$ & $24 \%$ \\
\hline Total & 143 & & \\
\hline No answer & 51 & & $26 \%$ \\
\hline
\end{tabular}

Table 12: Willingness to join a health prevention program

$50 \%$ of the participants did not answer the question or ticked "No". These participants could still answer the open question about health management or tick health management activities. This reduced the risk that a participant did not understand or misunderstood what is meant with health management activities.

Additional there were answers to the open question which provided qualitative data. There were in total above 30 comments. Multiple answers were possible. For categorizing, the open question was divided in two parts "yes" and "no". In the following table, the grouped answers for the questions are summarized. The qualitative part of the question for existing health management was designed as open questions. To use 
the comments as quantitative data the author has grouped them to comparable units. In the following table, the grouped answers for the first part of the question are summarized. It only includes the answer if the participant has ticked "Yes - There are health management activities in my organisation".

\begin{tabular}{|l|c|}
\hline \multicolumn{2}{|l|}{ Which health management program would you join? } \\
\hline Back training & 4 \\
\hline Movement (Jogging, Trekking, walking,) & 5 \\
\hline Wellness and Fitness & 5 \\
\hline Yoga and meditation & 7 \\
\hline Massage & 2 \\
\hline Nutrition program & 4 \\
\hline Stress reduction program & 2 \\
\hline Total & 29 \\
\hline
\end{tabular}

Table 13: Health activities mentioned in the open answer

\begin{tabular}{|l|c|}
\hline \multicolumn{2}{|l|}{ Why would you not join a health management program? } \\
\hline No time & 5 \\
\hline Organisation to small & 2 \\
\hline
\end{tabular}

Table 14: Reasons for refusal of health activities in the open answer

Five answers in both parts of the question were not included, because they were not linked to the questions. Alternatively, the answers were not readable.

The following questions are about different health management activities.

The quantitative data for health management programs with movement promotion is a multiple-choice question. Many of the participants ticked "no" they would not take part in health management (table above) but "yes" in several activities. The first table shows the number of ticks.

\begin{tabular}{|l|c|c|c|c|c|c|c|}
\hline Which programs for the activity "movement promotion" you would join? \\
\hline & yes & rather yes & depends & rather no & no & sum \\
\hline Training at the workplace (max. 10 min) & 43 & 38 & 52 & 30 & 17 & 180 \\
\hline Training at home (max. 10 min) & 26 & 64 & 44 & 34 & 11 & 179 \\
\hline Sponsored activity after work (Fitness, Yoga, etc.) & 49 & 62 & 38 & 23 & 13 & 185 \\
\hline With a counter for the steps or a fitness watch & 36 & 51 & 31 & 40 & 24 & 182 \\
\hline
\end{tabular}


Which programs for the activity "movement promotion" you would join?

\begin{tabular}{|l|c|c|c|c|c|c|}
\hline & yes & rather yes & depends & rather no & no & sum \\
\hline Training within a group of colleagues & 25 & 46 & 54 & 36 & 20 & 181 \\
\hline
\end{tabular}

Table 15: Choice of "movement promotion" programs total ticks

In the following table, the same data as above were used but in percentages.

\begin{tabular}{|l|c|c|c|c|c|}
\hline $\begin{array}{l}\text { Which activity of "movement promotion" } \\
\text { you would join? }\end{array}$ & yes & rather yes & depends & rather no & No \\
\hline Training at the workplace (max. 10 min) & $24 \%$ & $21 \%$ & $29 \%$ & $17 \%$ & $9 \%$ \\
\hline Training at home (max. 10 min) & $15 \%$ & $36 \%$ & $25 \%$ & $19 \%$ & $6 \%$ \\
\hline Sponsored activity after work (Fitness, Yoga, etc.) & $26 \%$ & $34 \%$ & $21 \%$ & $12 \%$ & $7 \%$ \\
\hline With a counter for the steps or a fitness watch & $20 \%$ & $28 \%$ & $17 \%$ & $22 \%$ & $13 \%$ \\
\hline Training within a group of colleagues & $14 \%$ & $25 \%$ & $30 \%$ & $20 \%$ & $11 \%$ \\
\hline
\end{tabular}

Table 16: Choice of "movement promotion" programs in percentages

The following table summarizes only the "yes" and "no" without a tendency "rather yes" or "rather no". The middle choice "depends" is carried over as the percentage of "neutral".

\begin{tabular}{|l|c|c|c|}
\hline \multicolumn{2}{|l|}{ Which programs for the activity "movement promotion" you would join? } \\
\hline & Yes & Neutral & No \\
\hline Training at the workplace (max. 10 min) & $45 \%$ & $29 \%$ & $26 \%$ \\
\hline Training at home (max. 10 min) & $50 \%$ & $15 \%$ & $25 \%$ \\
\hline Sponsored activity after work (Fitness, Yoga, etc.) & $60 \%$ & $24 \%$ & $19 \%$ \\
\hline With a counter for the steps or a fitness watch & $48 \%$ & $13 \%$ & $35 \%$ \\
\hline Training within a group of colleagues & $39 \%$ & $20 \%$ & $31 \%$ \\
\hline
\end{tabular}

Table 17: Choice of "movement promotion" programs reduced to "yes" or "no"

The quantitative data for health management program for weight reduction and change of nutrition is presented in the following tables. The first table shows the number of ticks for each activity and how they are rated. 


\begin{tabular}{|l|c|c|c|c|c|c|}
\hline \multicolumn{7}{|l|}{ Which activities for weight reduction and change of nutrition would you support? } \\
\hline & yes & rather yes & depends & $\begin{array}{c}\text { rather } \\
\text { no }\end{array}$ & no & sum \\
\hline $\begin{array}{l}\text { Would you join a group session with an ex- } \\
\text { ternal consultant? }\end{array}$ & 20 & 40 & 48 & 42 & 29 & 179 \\
\hline $\begin{array}{l}\text { Would you join a single session with an ex- } \\
\text { ternal consultant? }\end{array}$ & 26 & 58 & 45 & 27 & 24 & 180 \\
\hline $\begin{array}{l}\text { Would you join a session with a medical phy- } \\
\text { sician from your medical office? }\end{array}$ & 15 & 31 & 42 & 52 & 36 & 176 \\
\hline $\begin{array}{l}\text { Would you like to get healthy snacks/food at } \\
\text { work? }\end{array}$ & 106 & 47 & 15 & 7 & 8 & 183 \\
\hline
\end{tabular}

Table 18: Choice of "Weight reduction and change of nutrition" - total ticks

In the following table, the figures above are used to display the percentages of the answers.

\begin{tabular}{|l|c|c|c|c|c|}
\hline \multicolumn{6}{|l|}{ Which activities for weight reduction and change of nutrition would you support? } \\
\hline & Yes & rather yes & depends & rather no & no \\
\hline $\begin{array}{l}\text { Would you join a group session with } \\
\text { an external consultant? }\end{array}$ & $11 \%$ & $22 \%$ & $27 \%$ & $23 \%$ & $16 \%$ \\
\hline $\begin{array}{l}\text { Would you join a single session with } \\
\text { an external consultant? }\end{array}$ & $14 \%$ & $32 \%$ & $25 \%$ & $15 \%$ & $13 \%$ \\
\hline $\begin{array}{l}\text { Would you join a session with a med- } \\
\text { ical physician from your medical of- } \\
\text { fice? }\end{array}$ & $9 \%$ & $18 \%$ & $24 \%$ & $30 \%$ & $20 \%$ \\
\hline $\begin{array}{l}\text { Would you like to get healthy } \\
\text { snacks/food at work? }\end{array}$ & $58 \%$ & $26 \%$ & $8 \%$ & $4 \%$ & $4 \%$ \\
\hline
\end{tabular}

Table 19: Choice of "Weight reduction and change of nutrition" - percentages

The following table summarizes only the "yes" and "no" without a tendency "rather yes" or "rather no". The middle choice "depends" is carried over as the percentage of "neutral".

\begin{tabular}{|l|l|l|l|}
\hline Which activities for weight reduction and change of nutrition would you support? \\
\hline & Yes & Neutral & No \\
\hline Would you join a group session with an external consultant & $34 \%$ & $26 \%$ & $40 \%$ \\
\hline Would you join a single session with an external consultant & $47 \%$ & $25 \%$ & $28 \%$ \\
\hline
\end{tabular}




\begin{tabular}{|l|l|l|l|}
\hline \multicolumn{2}{|l|}{ Which activities for weight reduction and change of nutrition would you support? } \\
\hline & Yes & Neutral & No \\
\hline $\begin{array}{l}\text { Would you join a session with a medical physician from your medical } \\
\text { office }\end{array}$ & $26 \%$ & $24 \%$ & $50 \%$ \\
\hline Would you like to get healthy snacks/food at work & $84 \%$ & $8 \%$ & $8 \%$ \\
\hline
\end{tabular}

Table 20: Choice of "Weight reduction and change of nutrition" program - reduced to "yes" or "no"

After the questions on different activities which the participants would prefer, the last question was focused on vaccines.

Every question has a quantitative and qualitative part. The evaluation of the open question about vaccines revealed a possible problem within the questionnaire. The open question result about which vaccines are used leads to answers like "all" or a list of five or six vaccine names. For the evaluation, the author used a category for participants who gave a feedback which could be interpreted as all vaccines. This means if the participant wrote down five or six standard vaccines it was counted as fully vaccinated. There are typical seasonal vaccines like the seasonal flu vaccine, they were counted extra. Travel vaccines like the tick-borne encephalitis (TBE) or the anti-rabies inoculation were counted extra if they were mentioned.

In Germany, there is a group of people who are against vaccines in general. They do not believe in the effect of vaccines or belief they have a negative effect on the immune system. This discussion is more a discussion about beliefs and less about science. To avoid an ethical conflict or discussions with this group the author did not categorize answers to open questions, which were just against vaccines. There are ten comments like "I was not ill in the last years therefore I do not need vaccines" or "My immune system is strong enough". These comments were interpreted as a negative answer "No, I do not use vaccines".

The questions for vaccines are divided in the question for vaccines use in work and private life. The first question about vaccines was about the acceptance of vaccines in the private live.

\begin{tabular}{|l|c|c|}
\hline \multicolumn{3}{|l|}{ Do you regularly use vaccine in your private life? } \\
\hline & Total & Percentage \\
\hline Yes & 145 & $75 \%$ \\
\hline No & 37 & $19 \%$ \\
\hline No answer & 12 & $6 \%$ \\
\hline Total & 194 & \\
\hline
\end{tabular}

Table 21: Quantitative data on the use of vaccines in private life 
There were two answers with "no" vaccines in private life but in the open part "no vaccines" meant all ground vaccines but not the flu vaccine. These two answers were counted as a "yes" in the table above.

The following table summarizes the evaluation of the open questions for private life vaccines.

\begin{tabular}{|l|c|}
\hline \multicolumn{2}{|l|}{ Which vaccines do you use regularly in your private life? } \\
\hline & Total \\
\hline Flu vaccine & 38 \\
\hline No flu vaccine & 3 \\
\hline Tick-borne encephalitis & 38 \\
\hline All vaccines & 127 \\
\hline Total (Multiple answers were possible) & 206 \\
\hline
\end{tabular}

Table 22: Qualitative Data on the use of vaccines in private life

The vaccines for flu and tick-borne encephalitis were mentioned in the open question. The "no flu vaccine" means it was explicitly written down: "Yes, I use vaccines but no flu vaccine." For the other vaccines, there were no further comments.

The question about the possible use of vaccines provided by the employer was reduced to one question. There was only the choice for "yes" and "no" to generate the quantitative data.

\begin{tabular}{|l|c|c|}
\hline \multicolumn{3}{|l|}{ Would you join a vaccine program at work? } \\
\hline & Total & Percentage \\
\hline Yes & 171 & $88 \%$ \\
\hline No & 12 & $6 \%$ \\
\hline No answer & 11 & $6 \%$ \\
\hline Total & 194 & \\
\hline
\end{tabular}

Table 23: Quantitative data for vaccine program in work

The open question did provide some examples for vaccines:

\begin{tabular}{|l|c|}
\hline Would you join a vaccine program at work? & Total \\
\hline Flu & 39 \\
\hline No flu & 3 \\
\hline TBE & 25 \\
\hline
\end{tabular}




\begin{tabular}{|l|c|}
\hline Would you join a vaccine program at work? & Total \\
\hline All vaccines & 127 \\
\hline
\end{tabular}

Table 24: Qualitative data for a vaccine program in work

In the open question three participants wrote they would join a vaccine program, but they do not want the flu vaccine.

The question for the regular status check was not divided into private and work life.

\begin{tabular}{|l|c|c|}
\hline \multicolumn{3}{|l|}{ Do you check your vaccine status regularly? } \\
\hline & Total & Percentage \\
\hline Yes & 163 & $89 \%$ \\
\hline No & 20 & $11 \%$ \\
\hline No answer & 11 & \\
\hline Total & 194 & \\
\hline
\end{tabular}

Table 25: Quantitative data on vaccines status check

\subsection{Preferences of the MFAs on health-promoting measures}

The perspectives of the stakeholders are important for the improvement of health management. The results of the employees are important because the employees are the key of every health activity. Their acceptance for activities is needed; without the allowance of the employee and their agreement, no activity can be implemented.

According to literature review, it was obvious that there are no studies on health management in general practitioners' surgeries. This research and the generated information provide additional information about this group of employees, the MFAs, and can help to change the health management in these organisations. The research findings in this point are central for the development of a priority concept - the subject of the next chapter.

In the first step, the gender and the age group of the MFAs are examined more closely. The following subsection is a closer look at the general medical practice from the point of view of "health management". The focus is on the measures that should be included in a priority concept. For these measures, preference profiles are developed.

\subsubsection{Gender and age groups of the MFAs}

Two demographically relevant characteristics were recorded: the gender and the age group. 
As already stated in subsection 2.2.2, the MFA profession with a woman share of $98.3 \%$ is a typical women's occupation. This is confirmed by the MFA survey, which according to Table 6 shows a woman share of $100 \%$. For this research, it was therefore only necessary to examine whether the share of $100 \%$ is a random result or whether this proportion is in the so-called 95\% confidence interval of the "true" value $98.3 \%$. With the aid of the formula proposed by Pernerstorfer (2009), the lower limit of $96.4 \%$ was determined for sample size 194 and $100.0 \%$ for the upper limit. That is, the proportion of women found is not a random result; rather, it is significant at a confidence interval of $95 \%$.

This result is not surprising, but it is important to keep in mind all the generalizations that may be derived from this research, that all statements refer to the groups "women". Thus, the following comparison of the age groups also refers to the female proportion of the employed.

The following diagram uses the data from Table 7 "Age of the MFAs" and compares it with two other available statistics. The blue columns are the percentages of the respective age group from this MFA survey. The data from the orange columns is from the Federal Bureau of Statistics (destatis, 2016) and shows the distribution of the female labour force in the year 2016. The grey column data were taken from the government report "Health Care Data" (BMG, 2016), which identifies age groups for the occupational group "doctor's receptionists". As there is no official data on the age structure of the MFAs, the data of the group of "doctor's receptionists", which are one third larger, were used. The data are from the year 2014. The latter two statistics use a different age structure, which has been adapted for this research work for reasons of better comparability.

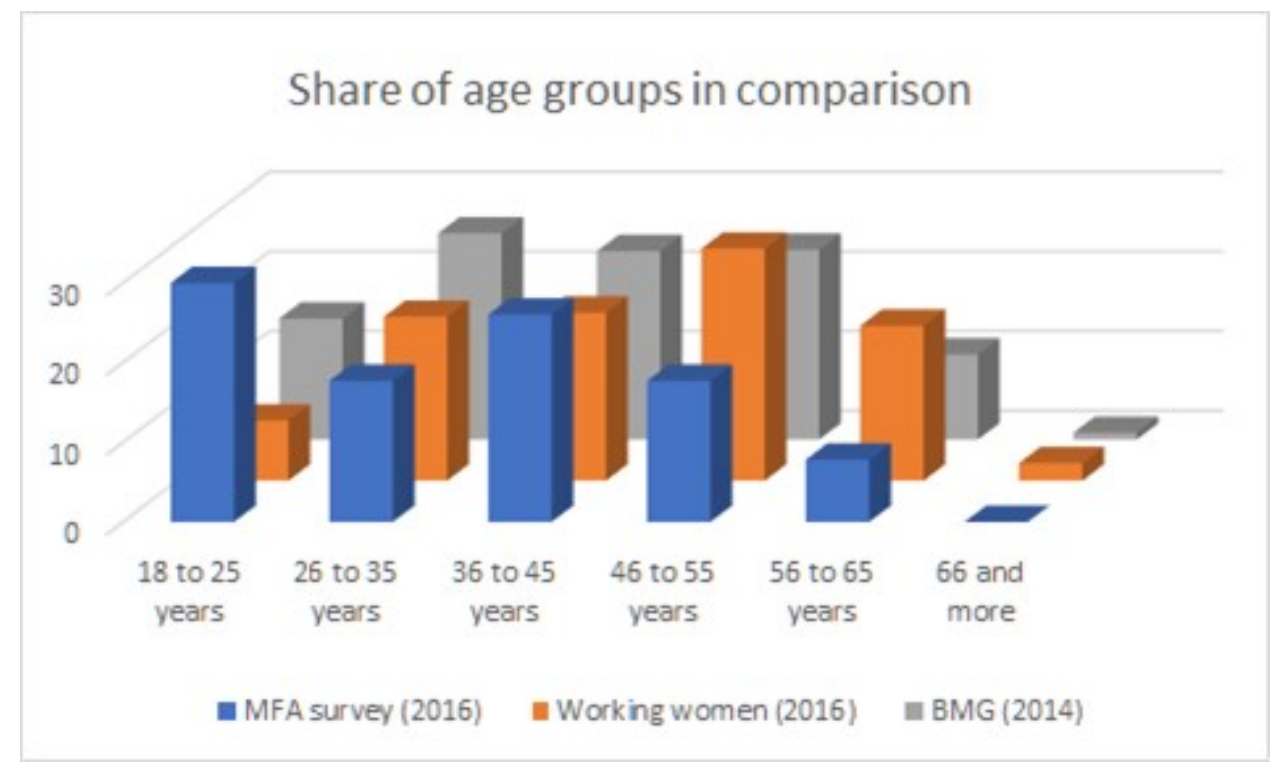

Figure 19: Age structure of the MFAs in comparison

At first glance, the highest share with $31 \%$ is the age group $18-25$ years. On the one hand, this may be because younger employees may be sent to trainings rather than 
older ones, and on the other hand, the high popularity of the MFA vocational training: With 15,822 newly concluded training contracts, in 2016 the MFA was one of the most popular vocational training programs for women (Rank 2). If one adds to the general MFAs the dentists' specialized MFAs (with 12,780 Rank 6), the medical assistants are the most popular profession regardless of gender - before "Office manager" (28,656 contracts) and "Management assistant in retail" (25,191 contracts) (BIBB, 2016).

The proportion of the age group 26-35 years is the second lowest. This could be because for a typical female professor at this age, the phase of the family foundations starts and many women in the family place higher priority, especially since they are often "only" the second earners. On the other hand, it could be a sign that the expectations of the profession are not fulfilled, and the exit rate increases. For example, Kathmann and Dingeldey (2013, p. 12) reported an exit rate of up to 54.6\% over 15 years.

In addition to the peak of the youngest group, the age group 36 to 45 years has the highest share. This is untypical in comparison to the age structure of female workers. Among these, the largest proportion is in the age group 46 to 55 years. This may be an indication that the medical assistance professions will not be so severely affected by demographic change.

The share of the age groups 46 to 55 years and 56 to 65 years then decreases again and above average. Unlike the younger ones, the lack of (or realized) career opportunities are a plausible reason for leaving the professional group of MFAs. The same trend, albeit less pronounced, can be seen in the group of doctor's receptionists.

In the overall picture, unlike the general labour market for women, the proportion of older people does not increase, but decreases from about 35 years. This not only focuses on the aging problem but also the attractiveness of the occupational field for health management measures. For the role of the physician as a leader, the age structure proves to be ambivalent. On the one hand, as long as the MFAs are one of the most attractive and sought-after occupational profiles, the doctors can rely on the fact that no job placement problems occur. On the other hand, the attractiveness of the MFA jobs cannot be indifferent to them as dissatisfied employees without a career chance causes failures and absent times (Vu-Eickmann and Loerbroks, 2017).

The issues related to the age show that MFAs do not face any demographic change problems compared to other occupational groups. In this section the age structure is used to check whether the measures referred to as preference require age groupspecific adjustments. 


\subsubsection{Experience with and willingness for health management}

The question about the specialisation of the medical physician was important to check if the target group completed the questionnaire. It was used to prove if the main employees who completed it are really working in general practitioners' surgeries. From the participants who provided information about the kind of their medical offices, $92 \%$ are working in general practitioners' surgeries. The rest are working in specialist practices, which are organized in a comparable way.

In the questionnaire $8 \%$ of the participants work for other specialised surgeries. The female quote there is $100 \%$ too and it may be that the activities, which are preferred by the employees of general practitioner, could be transferred. This point is addressed under "Conclusions".

There is a quote of general practitioners' surgeries that already use health management program. $26 \%$ (Table 9) have a program for health management. Such as vaccine programs or a regular health check-up.

To interpret the results of the questionnaire it was necessary to know how many SMEs or general practitioners' surgeries use an implemented health management system. The literature review identified one study of higher interest in this context. The results of „Health Management in Small and Medium-Sized Enterprises” (Zelfel, Alles et al., 2011) argue that there is a need to improve the health management in German SMEs and that the health management is not established in the German SMEs. The study provides a revealing figure about the dissemination of health management. The authors made telephone interviews with 1,441 organisations between 1 and 250 employees in seven regions of Germany. The result concerning the usage of health promotion activity was that $20 \%$ of the contacted organisation has made some kind of health management.

The result from the questionnaires is that $26 \%$ of the general practitioners' surgeries have a health management program. This means that the quote of health management in general practitioners' surgeries compared with the figures provided by Zelfel, Alles et al. (2011) is 23\% higher than in SMEs in general. This is a significant difference to the average which was identified in the research "Health Management in Small and Medium-Sized Enterprises - Results of a Representative Survey“ (Zelfel, Alles et al., 2011).

However, this is a positive result for the general practitioners' surgeries. There is a higher level of health management and there is potential for improvement. It is fascinating why this quote is higher and if this could be used for the implementation of health management. The questionnaire of this research was completed in between end of 2015 and the beginning of 2016. The other one was finished in 2011. The idea of health management was promoted by many congresses, medical associations, management associations, the government and insurance companies in the last years. This would mean that there is a general positive trend for health management. To 
prove this, actual studies with figures from 2014 to 2015 would be needed to compare it.

The research from Zelfel, Alles et al. (2011)(Zelfel, Alles et al., 2011) is representative this could be a reason too. This research is not representative. It is possible but unlikely that this is the explanation for the difference.

Another reason may be the size of the selected companies. The research from Zelfel, Alles et al. (2011) includes SMEs without any boundaries. The definition of SMEs is a wide range from one employee to 250 employees.

A weak point is that there is no clear definition of a health management system. The research identified several activities for health improvement; however, the literature research did not provide a definition of a health management system. The author could identify if these participating companies have implemented health management activities but not define the health management system. Buying an ergonomic chair could be a health supporting activity, but it is no health management system.

The results of the open questions are important in this case, they provide an insight into the existing health management in the general practitioners' surgeries. Compared to "Health Management in Small and Medium-Sized Enterprises - Results of a Representative Survey“ (Zelfel, Alles et al., 2011) this open question allowed the author to identify the kind of health management which is used. This is the first research for health management in general practitioners' surgeries. There are no data on which health management activities are already in use in these organisations.

The categorized results from the open questions could be compared with the results from the multiple choices questions. Table 10 "Existing health management activities for the open question "yes" provide a summary of the open questions. By the literature review, the author gained an in-depth insight which activities are common for effective health management. However, the results of the open questions provided additional information about activities, which were not identified in the literature review.

Over $30 \%$ of the participants who completed the open questions stated that there are check-ups in their organisation. In the literature research, there were no results for general check-ups and their efficiency. If the health insurance overtakes the costs this could be an effective and cheap activity for an organisation. Check-ups with medical proven effects are provided in Germany by several health insurances for free to motivate their clients to go to a physician when they are healthy, before they are ill. This is used as a prevention to identify possible risk factors like overweight and to avoid them. There are several check-up programs for prostate cancer, breast cancer and other further diseases. In Germany, this is a political decision which is in the wider area of health management. However, the acceptance of this activity would be presumably low. The participant could write down the activities they prefer in the open questions for preferred health management. However, the qualitative data on this 
show that none of the participants would prefer a check-up. One explanation for the existing health check-ups could be financial. In the case of general practitioners' surgeries, the physicians can invoice it to the health insurance and earn money with their own employees.

In total, the answers to the open questions confirm the selection of the multiplechoice questions through the literature review. The selection involves nearly all activities which are still in use. For the implementation of the priority list, this is helpful. When a general practitioner already uses health management techniques, they can use such activities which are not implemented.

This part of the result provides insight if the employees have a positive or negative attitude to the health management. With $63 \%$ acceptance rate a clear majority of the employees would accept a health management program. This acceptance is important for the practical use of the activities. The employer knows that their employees would like to take part.

A quote of participants did not tick "yes" or "no" for the general question. This group may be undecided, and it could be possible to motivate them to participate in activities. Another effect is that the participant originally ticked that they would not take part in a health management program. Afterwards they ticked at which health management program in their organisation they would take part. This is paradox. An explanation would be that the participant did not know what a health management program is. For example, the yearly flu vaccine is a health management activity, but for the employees it is just the normal yearly flu vaccine. In this case they would tick "no" for join a health management program and "yes" for the vaccine.

The null hypothesis, formulated in subsection 4.2.7, concerning the willingness to participate in a health promotion program was as follows:

$H_{0}$ : The willingness of the MFAs to participate in a health promotion program is independent of age.

The following table allows the hypothesis test:

\begin{tabular}{|c|c|c|c|c|}
\hline Age & yes & no & $\begin{array}{c}\text { Expected } \\
\text { „no“ }\end{array}$ & p-value \\
\hline $18-25$ & 23 & 17 & 18.0 & 0.8100 \\
\hline $26-35$ & 20 & 7 & 15.7 & 0.0285 \\
\hline $36-45$ & 28 & 40 & 21.9 & 0.0001 \\
\hline $46-55$ & 18 & 10 & 14.1 & 0.2746 \\
\hline $56-65$ & 8 & 2 & 6.3 & 0.0882 \\
\hline & 97 & 76 & 76 & $\mathbf{0 . 0 0 0 1}$ \\
\hline
\end{tabular}

Table 26: Hypothesis test: Willingness to participate is independent of age 
In the rows of the table are the age groups, in the columns "yes" and "no" are the number of ticks in the questionnaires for the willingness to participate or not. In the column "Expected no" is the calculated number of "no", assuming the null hypothesis holds, and you can expect the same distribution for "yes" and "no" answers. For example, in the age group 26-35 the answer "yes" was given by 20 of 97 persons. That means $20.6 \%$. This percentage applied to the total number 76 of "no"-answers leads to expected 15.7 persons.

The chi square test calculates the differences between the actual number and the expected number. From this, the significant level is determined as the probability that the actual and the expected number are distributed in the same way, the p-value. In this case the $\mathrm{p}$-value is $0.01 \%$.

Since the p-value is less than $1 \%$, the null hypothesis can be rejected and the counter hypothesis

$H_{1}$ : The willingness of the MFAs to participate in a health promotion program is not independent of age.

must be accepted on a confidence level of $99.9 \%$. If the chi square test is applied to each age group, the reason becomes clear. The main deviation between real number and expected number of "no" is the age group 36-45 years with the p-value less than $1 \%$ and the age group 26-35 years with a p-value less than 5\%. As visualized in Figure 20. This means e.g. that an institution wants to increase the willingness to participate for example by means of information materials or information events, would need to make special arrangements for the middle-aged group of MFAs to motivate them.

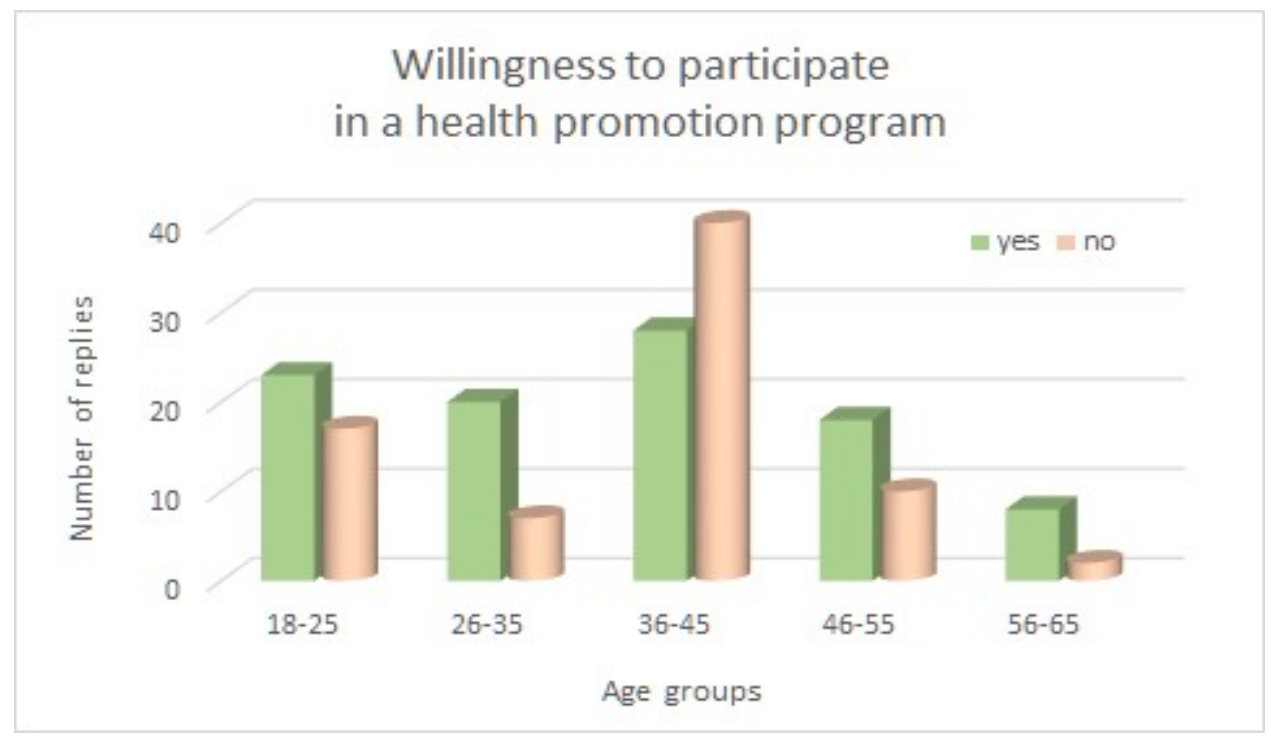

Figure 20: Different willingness to participate in health-promoting measures 
The next finding concentrates on the occurrence of the combination of the items "Experience" and "Willingness". 143 participants responded to the question of existing health care activities as well as about the willingness to participate.

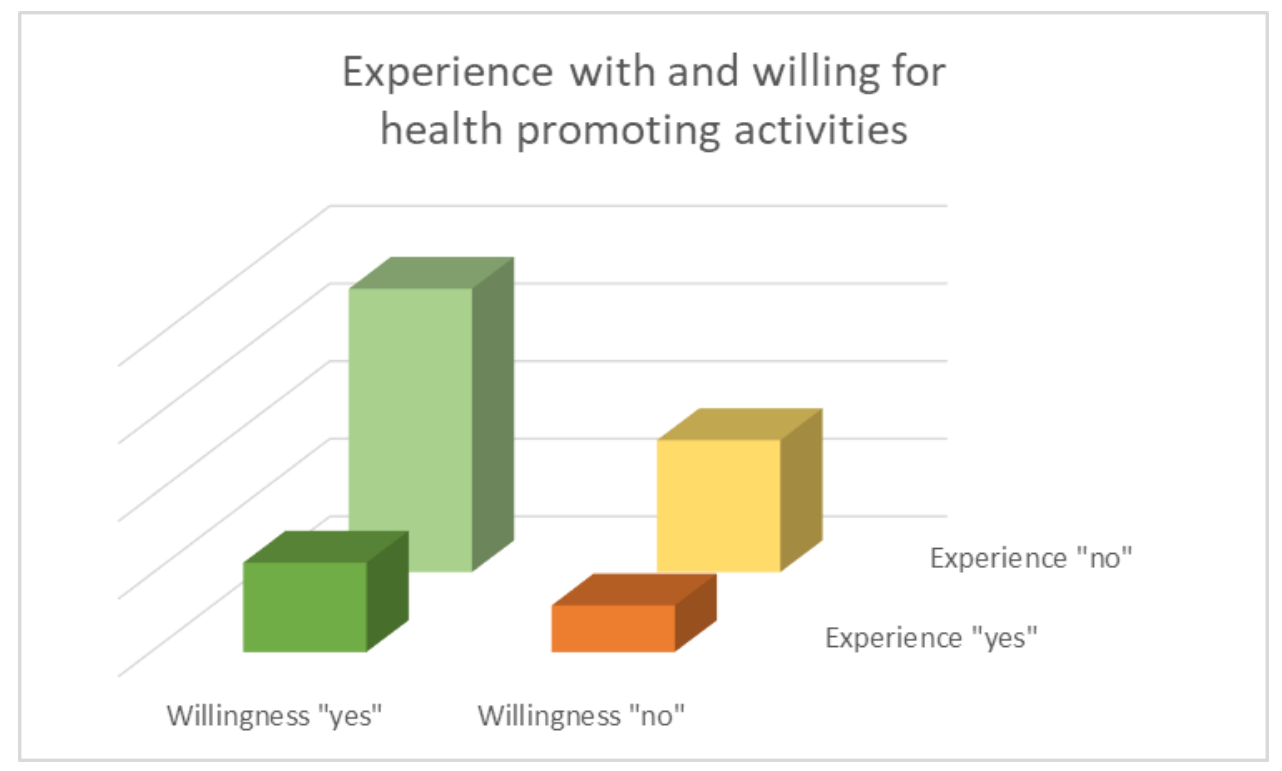

Figure 21: Group formation with the combination "experience" and "willingness"

The compilation of the response combinations reveals four clearly distinguishable groups as presented in the figure above. To clarify the differences, the four groups were labelled by the author with four most succinct names.

- The "curious optimists" (51\%): with no experience but willing to join - They form by far the largest group. They are by far the largest group and most likely they expect the greatest effect on their personal well-being.

- The "cautious pessimists" (24\%): with no experience and (hence) not willing to join - They form the second-strongest group and answered in the previously described paradoxical way and often called "no one cares" and "no time" as possible obstacle grounds. This group is likely to be targeted through detailed information work for health promotion activities.

- The "experienced optimists" (16\%): with experience and (therefore) ready for further participation - They make a large part of the 40 responses to already existing measures and seem to be so satisfied with the past so far that they would participate in further measures.

- The "disappointed" (8\%): with experience and (therefore) the lack of willingness to further participation. - The relative low percentage is not necessarily against the acceptance of the measures; because it is not clear whether other reasons (e.g. family circumstances) were decisive. These MFAs also replied in the paradoxical manner described above. In particular, the proposed measures for the promotion of movement and nutritional advice did not appear in the open responses of this group. 
The following subsection examines, inter alia, whether there are different preferences for the individual programs proposed for the group of "experienced" and "willing".

\subsubsection{Preference profile to programs of movement promotion}

Taking all five proposed activities together, the acceptance of "movement promotion" is good. Following Table 17 the most popular health promotion activity with $60 \%$ of the nominations would be the support of private activities like fitness or yoga. This is not a surprise, because the employee could keep on their private sports and get a part of it paid. This acceptance could be used to motivate employees who do not take sports at home to start with it. The next activities are closer together: $50 \%$ would join training at home and $45 \%$ a daily training program in the office. Training with a step counter or a fitness watch is between these values and is be advocated by $48 \%$. The "Training within a group of colleagues" with $39 \%$ is on the last rank.

In order to gain a deeper insight into the preference structure of MFAs for movement promotion programs, the Likert (1932) scales (1 for "yes" up to 5 for "no") were evaluated for each proposed activity by age group.

The following table shows this as an example for the activity "Training at workplace (max. $10 \mathrm{~min}$ )". Prior to the evaluation, it was ensured that the ticks were not created by chance or by arbitrary ticking. For this purpose, the two null hypotheses "The Likert (1932) scale values for training at workplace are equally distributed" as well as "The Likert scale value for training at workplace are normally distributed" were tested for significance. Equally distributed would mean that each of the five values would be about the same number, i.e. about $20 \%$, the answer would be "diced". Normal distribution would mean that the participants most often cross the mean value (3 - neutral) and the extreme values ( 1 - yes and 5 - no) do not.

The null hypotheses for normal distributions can be discarded $(\mathrm{p}<0.1 \%)$ so that the counter hypotheses "The answers are not normal distributed" are confirmed as "highly significant". The null hypotheses for uniformity distribution are rejected for "sponsored activity", "training at home" and "step counter or fitness-watch" with $\mathrm{p}<0.1 \%$, so that the counter hypotheses "The answers are not equally distributed" are also as "highly significant" are accepted. The p-value of $1.6 \%$ for "Training within a group of colleagues" and the p-value of $2.2 \%$ for "Training at workplace" means that the counter hypotheses are classified as "very significant".

The following table shows the 5 age groups in the columns. The last column contains the sum, the average or the standard deviation (SD) depending on the line. The first line shows how many responses for the "Training at workplace" measure was applied to the "yes" in the respective age group, For example 10 in the age group 36 to 45 years. At the end of the line the sum is indicated. The lines for the different values of the Likert scale are followed by the total line for the selected activity, here "Training at workplace". For example, that this measure was answered by 57 participants of the 
age group 18 to 25 years. The following is a line with the average values for the respective age group. The average value is given by the Likert (1932) scale. It should be noted that a smaller average value means a higher preference. An average less than " 3 - neutral" means that the preference for the measure tends to be "yes". In the example, the "training at workplace" measure is most preferred by the age group "56 to 65 years". The average of all age groups - here 2.6 - is entered in the last column. The last line shows, how much the average of the age group deviates from the total average of the group up or down. In the example, the measure is rated above average with a good -0.7 deviations from the age group "56 to 65 years".

\begin{tabular}{|c|c|c|c|c|c|c|}
\hline $\begin{array}{l}\text { Age groups / } \\
\text { Likert scale }\end{array}$ & $\begin{array}{c}18 \text { to } 25 \\
\text { years }\end{array}$ & $\begin{array}{c}26 \text { to } 35 \\
\text { years }\end{array}$ & $\begin{array}{c}36 \text { to } 45 \\
\text { years }\end{array}$ & $\begin{array}{c}46 \text { to } 55 \\
\text { years }\end{array}$ & $\begin{array}{c}56 \text { to } 65 \\
\text { years }\end{array}$ & $\begin{array}{c}\text { sum } \\
\text { average/SD }\end{array}$ \\
\hline $1-$ yes & 11 & 5 & 10 & 7 & 4 & 37 \\
\hline 2 - rather yes & 14 & 4 & 8 & 10 & 3 & 39 \\
\hline $3-$ neutral & 21 & 11 & 14 & 5 & 3 & 54 \\
\hline 4 - rather no & 7 & 9 & 8 & 8 & 0 & 32 \\
\hline $5-$ no & 4 & 1 & 7 & 4 & 0 & 16 \\
\hline Training at workplace & 57 & 30 & 47 & 34 & 10 & 178 \\
\hline Average & 2.6 & 2.9 & 2.9 & 2.8 & 1.9 & 2.6 \\
\hline Deviation & 0,0 & 0.3 & 0.3 & 0.2 & -0.7 & 0.4 \\
\hline
\end{tabular}

Table 27: Preferences and age groups for "Training at workplace"

The illustration of the table as a bar chart shows again clearly the different assessment of the measure by age group. While it is most favoured by the oldest group of people, the other age groups tend to prefer it less favourably.

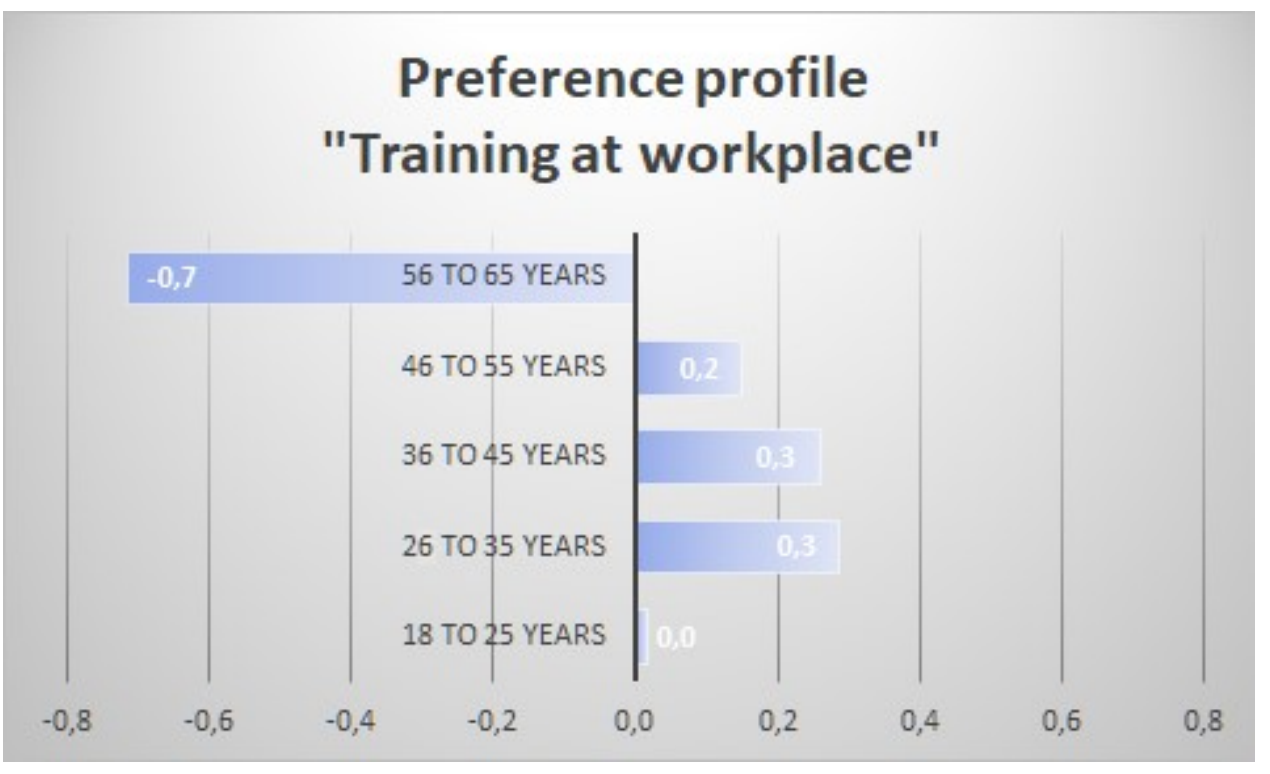

Figure 22: Differing preferences between the age groups

Such preference profiles can be applied for each of the proposed measures for the promotion of movement. If the deviation is not attributed to the overall average of a 
measure (2.6) but to the average for all measures (2.7), the overall profile is differentiated according to type of measure and age group.

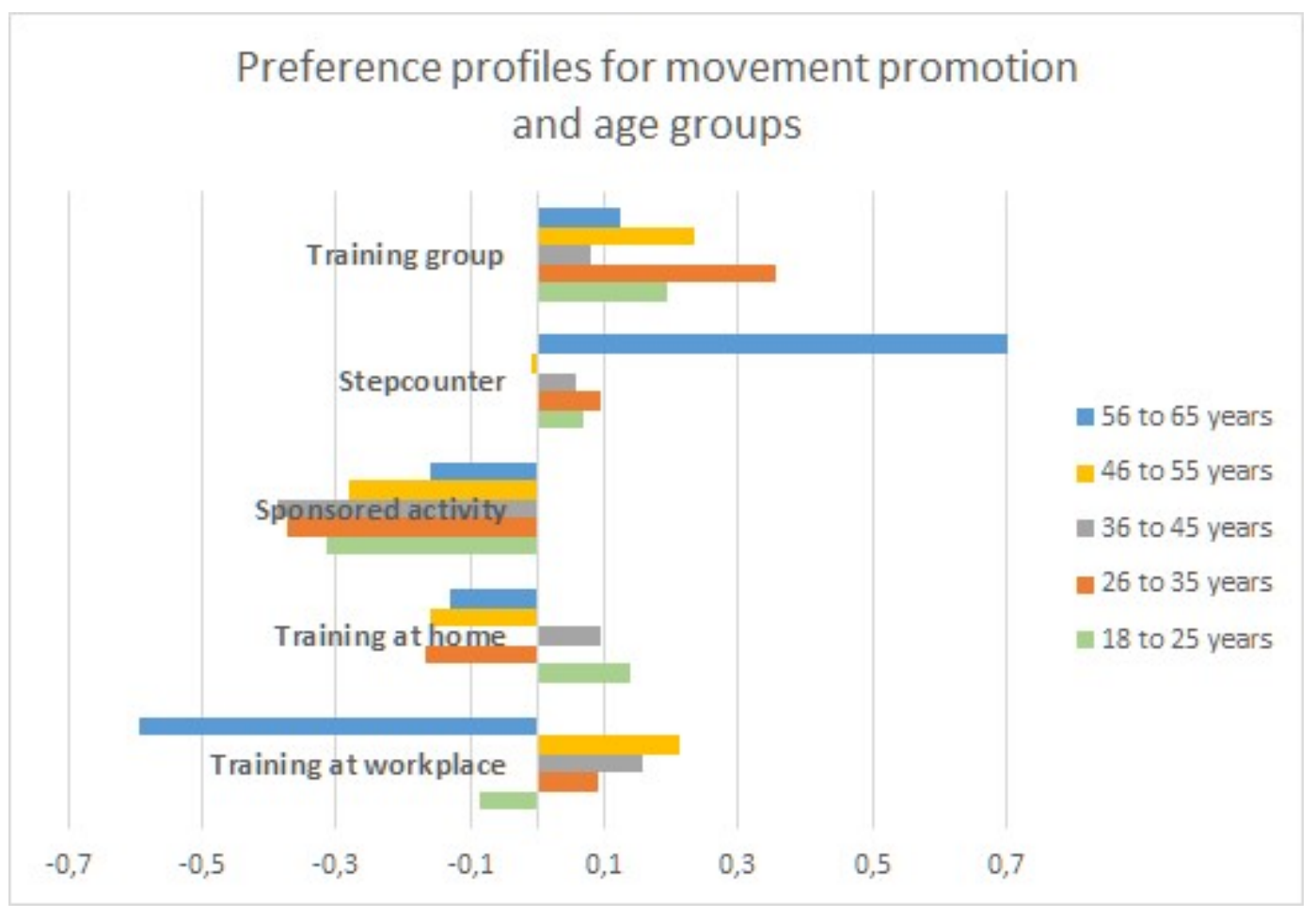

Figure 23: Preferences for the different measures of movement promotion and age groups

\subsubsection{Training in a group with colleagues}

As already known, this offer is the most unpopular (average: 2.9 - standard deviation: 0.2 ). It is the only measure that tends to "rather no" in all age groups. The age group 26 to 35 is the most disinclined. One possible explanation is that it rather avoids sports activities in an age-mixed group. For this explanation, the same group prefers the sponsored activities most strongly.

$H_{0}:$ The preference value of the age group 26 to 35 for the measure "Training in a group with colleagues" does not deviate from the other age groups.

The hypothesis test discards the null hypothesis $(\mathrm{p}<5 \%$ ) so that the hypothesis can be assumed to be significant.

In terms of experience, the measure is more likely to be statistically highly significant $(\mathrm{p}<1 \%$ ) of experienced MFAs (average 2.6) rather than inexperienced (average $3.0)$.

As is to be expected, the preference of MFAs, which have indicated their general willingness to participate in health-promoting measures, tends to be better than for the "unwilling" (average 2.6 resp. 3.6)

\subsubsection{Step counter or fitness-watch}


This training program is also perceived as being below average by almost all age groups (average: 2.9 - standard deviation: 0.3). The significant deviation in the age group is strikingly 56 to 65 years, while all other age groups evaluate this training form almost equally. A possible explanation would be that the older MFAs are not accustomed to using training measures with timings. As the hypothesis-test show the null hypothesis

\section{$H_{0}$ : The preferences of the age groups 46 to 55 and 56 to 65 do not differ}

can be rejected at a high level $(p=3.7 \%)$. Since the age group 45 to 54 shows a very slight deviation direction to "rather yes", the following null hypothesis

$H_{0}$ : The preference value of the age group 46 to 55 for the measure "Step counter and Fitness-Watch" does not differ from the other age groups.

was also tested. If you do not want to be satisfied with a confidence level of $90 \%$, the p-value of $8 \%$ is not sufficient to reject the null hypothesis.

Under the aspect of "experience", the measure is more likely to be statistically highly significant $(\mathrm{p}<1 \%$ ) by inexperienced MFAs (average 2.8 ) rather than by experienced (average 2.9)

As is to be expected, the preference of MFAs, which have marked their general willingness to join health-promoting activities, tends to be better than that for the "unwilling" (average 2.5 resp. 3.4).

\subsubsection{Sponsored activities}

This offer is most preferred by all MFAs and is uniform across all age groups with the lowest standard deviation compared to the other offers (average: 2.4 - standard deviation: 0.1). Since the middle age groups showed the highest approval in this offer, it was tested whether these values are statistically significant.

$H_{0}$ : The preferential value of age group 26 to 45 for the "Sponsored activities" measure does not differ from the other age groups.

The significance level of $95 \%$ shows that the thesis cannot be rejected or accepted.

In the "experience" aspect, there is no significant ( $p>5 \%)$ difference in the preference estimation between MFAs with (average 2.3) and without experience (average 2.4).

As is to be expected, the preference of MFAs, which have indicated their general willingness to join health-promoting activities, tends to be better than that of the "unwilling" (average 2.1 resp. 3.1).

\subsubsection{Training at home}

This offer is the second most popular offer. Remarkable are the slight deviations both upwards and downwards (average: 2.6 - standard deviation: 0.2). At least this offer is 
at least preferred by the youngest age group. In this context, the basic principles of younger people may be that they do not want their leisure time to be influenced by company initiatives.

$H_{0}$ : The preference value of age group 18 to 25 for the "Training at home" measure does not differ from the other age groups.

tested. The significance level of $13.5 \%$ shows that the thesis cannot be rejected or accepted.

In the "experience" aspect, the measure is statistically highly significant $(\mathrm{p}<1 \%)$ of experienced MFAs (average 2.3) more preferred than of inexperienced (average 2.8). At 0.5 , the range is greatest in the preference evaluation.

As was to be expected, the preference of MFAs, which have struck their general readiness for health promotion measures, tends to be better than that for the "unwilling" (average 2.5 resp. 3.3)

\subsubsection{Training at workplace}

This offer polarizes most. It has the largest span with -0.6 up and +0.2 down (average: 2.6 - standard deviation: 0.4). In their approbation the oldest group and the youngest group agree, albeit to varying degrees. Here, too, the basic principle could be expressed that the leisure time should not be influenced by company initiatives and that what can be done at the workplace should also be done there.

$H_{0}$ : The preference value of the youngest and oldest age group for the "Training at workplace" measure does not differ from that of middle age groups.

The hypothesis test confirms the contra hypothesis with $\mathrm{p}<1 \%$ as very significant.

In the "experience" aspect, the measure is more likely to be statistically highly significant ( $\mathrm{p}<1 \%$ ) of experienced MFAs (average 2.5) rather than inexperienced (average 2.8).

As was to be expected, the preference of MFAs, which have marked their general readiness for health-promoting measures, tends to be better than that for the "unwilling" (average 2.5 resp. 3.2)

\subsubsection{Preference profile to programs of nutrition counselling}

Following Table 20 the activities for "healthy snacks/food at work" are the most popular among in the group of nutrition (84\% approval with "yes" or "rather yes"). Afterwards all other nutrition activities are under 50\% acceptance. The next activity in the list is a "single meeting with a nutrition consultant" $(47 \%)$ or a group meeting (34\%). The lowest preference is reached by an "Internal session with a physician from the medical office" (26\%). 
The meeting of a nutrition consult within a group was voted with only $34 \%$ which can be interpreted as denial. An explanation may be that the MFAs do not want to discuss their nutrition or potential problems of overweight in a group with colleagues. These activities may be more cost effective because only one nutrition consultant must be paid for a group meeting. The acceptance is low and there is no hint in the literature review that in this case group sessions are more effective. The study "Incentives for smoking cessation." (Cahill, Hartmann-Boyce et al., 2015) found evidence that team meetings could have a positive effect, but there is no proof that it could be transferred from smoking to addiction or overweight.

The last suggested activity "Internal session" is most unpopular within the multiplechoice questions. It seems that the MFAs do not want her/his employer, in this case the medical physician as consultant for nutrition. This could have several reasons e. g. they do not want to talk to their employer about personal health or they do not trust in the qualification: Generally, a medical physician needs an additional qualification for nutrition consulting or must visit special education events. The German association for physicians provides rules for nationally standardised advanced training regulations and physicians can make the certification for nutrition consulting. Compared to the area of movement, general practitioners on the topic of "nutrition" have less consultancy competence. According to Figure 3, 42\% of the general practitioners estimate their expertise in "physical movement" very well, while the rate for "nutrition counselling" is only $37 \%$.

However, for all nutrition activities the employer has to invest money to provide an activity. Beside the health management aspect, the offer of healthy food and nutrition consulting could help to motivate employees. The studies with suggested activities which were identified in the literature review are not very clear about what explicit activity will have a positive effect. The World Health Organisation (Engbers, 2007) argues that workplace programs for improving eating conditions are generally accompanied by positive effects, even though these effects are moderate. Through nutrition consulting or fruits, the employee's consumption of fruit, vegetables, and fat could be influenced. Consultants or other informational strategies can encourage the consumption of healthier food and to provide healthier food during working hours can be a positive contribution. The research "A site-specific literature review of policy and environmental interventions that promote physical activity and nutrition for cardiovascular health: what works?" (Matson-Koffman, Brownstein et al., 2005) states that there is strong evidence in this context. However, the employees would prefer fresh and healthy snacks at work. For the employer, this will cause higher costs, but it helps to improve the health management and is accepted by the employees. Additionally, the workplace conditions are improved.

In order to gain a deeper insight into the preference structure of MFAs for programs of nutrition counselling, the Likert scales (1 for "yes" up to 5 for "no") were evaluated for each proposed activity by age group. 
As with movement promotion programs prior to the evaluation, it was ensured that the ticks were not created by chance or by arbitrary ticking. For this purpose, the two null hypotheses "The Likert scale values for weight reduction and change of nutrition activities are equally distributed" as well as "The Likert scale value for weight reduction and change of nutrition activities are normally distributed" were tested for significance.

The null hypothesis for normal distributions can be discarded $(\mathrm{p}<0.1 \%)$ so that the counter hypotheses "The answers are not normal distributed" are confirmed as "highly significant". The null hypothesis for uniformity distribution is rejected for "healthy snacks" with $\mathrm{p}<0.1 \%$, so that the counter hypotheses "The answers are not equally distributed" is to assume. The p-value of $0.5 \%$ for "single session with external consultant" means that the counter hypotheses is classified as "very significant". The "internal session" with a p-value of $1.7 \%$ is also to be rejected. Only in the case of "group meeting with a nutrition consultant" cannot be excluded at the level of significance of $5 \%$ that the answers are equally distributed, that is, they may have happened randomly.

The proposed activities for weight reduction and change of nutrition are less popular than the measures for the promotion of movement, except "healthy snacks".

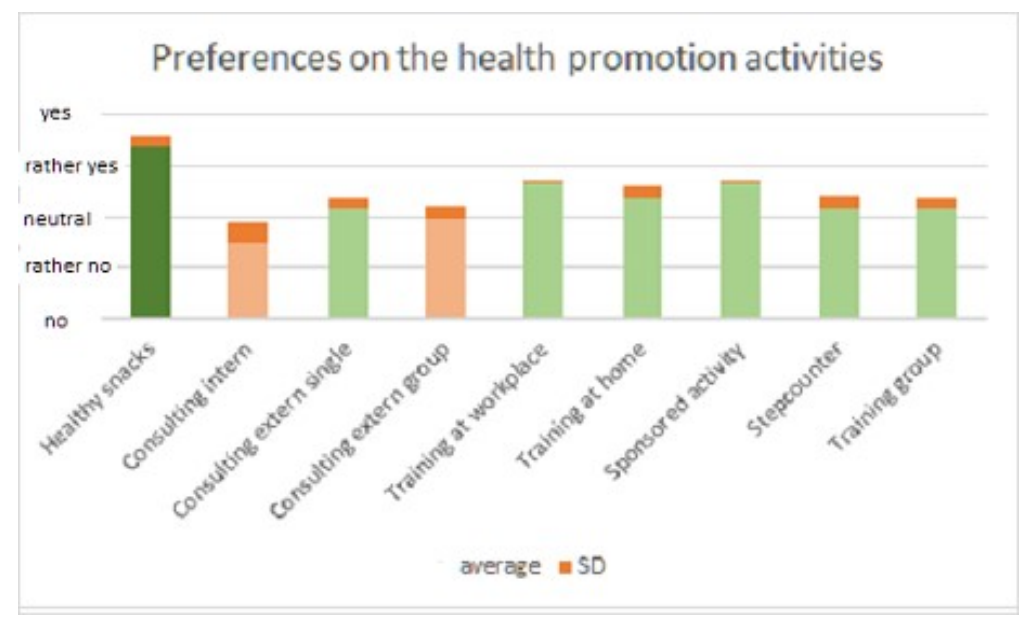

Figure 24: Evaluation of the Likert scale for all proposed activities

For the bar chart the weighted averages were calculated for each activity. The popularity on the Likert Scale ("yes" to "no") can be read directly from the entered lines. Average values better than "rather yes" were dark green. Better than "neutral" was lime green coloured and values under "neutral" were pale red. In order to indicate possible variations over the age groups, each column was supplemented by the simple standard deviation. (Approx. 95\% of all values are within $\pm 2 \mathrm{SD}$ ).

The bars on the left of the diagram contain the food-related activities. In attractiveness, they are clearly below the movement-related activities on the right side. In order to find possible causes, a preference profile was created by age group for the ac- 
tivities. The following figure enables to find possible differences in preference depending on age.

The preference profile (Figure 25) shows in the bars the deviation of the age group average from the average of the activity. Thus, the age group 56-65 assesses all nutritional activities on average with 2.2. The "Healthy Snacks" activity is rated better by this age group by -0.9 , i.e. by 1.3 .

\subsubsection{Healthy snacks}

Across all measures and all activities considered, "Healthy Snacks" is rated by the age group 56-65 years with 1.3 the best result at all. (1.0 would be the theoretical maximum). This may be since with increasing age many people recognize the value of healthy nutrition. Yet statistically significant is the null hypothesis

$H_{0}$ : The preference value of the age group 56 to 65 for the "Healthy Snacks" measure does not differ from the other age groups.

with a $\mathrm{p}$ value $>5 \%$ not, so that the null hypothesis can neither be rejected nor confirmed.

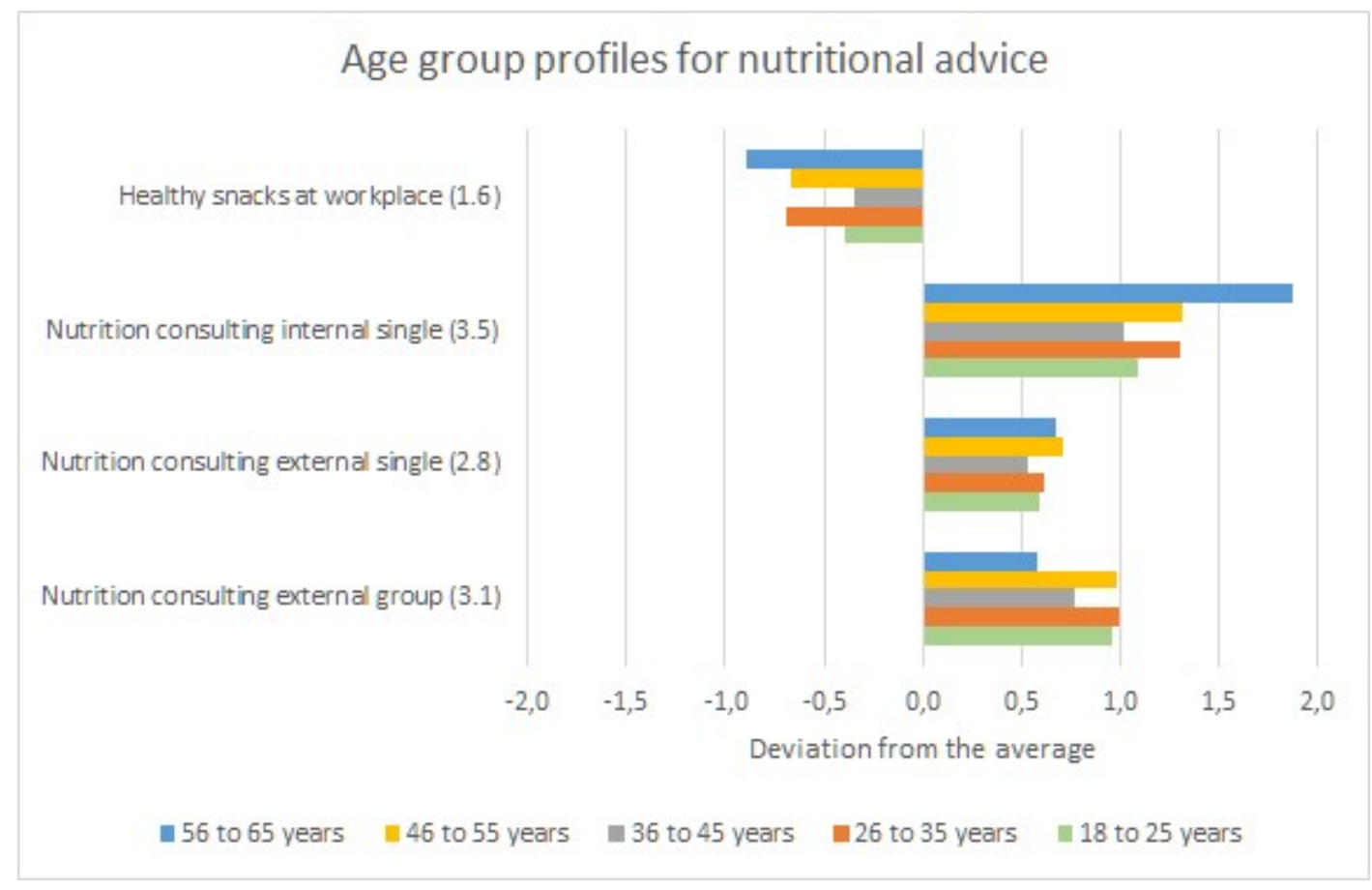

Figure 25: Preferences for the different measures of weight reduction and change of nutrition and age groups

Under the experience aspect, there is no significant ( $\mathrm{p}>5 \%$ ) difference in the preference estimate between experienced MFAs (average 1.7) and inexperienced (average $1.7)$.

As was to be expected, the preference of MFAs, which have marked their general readiness for health promotion measures, tends to be better than that for the "unwill- 
ing" (average 1.5 resp. 2.0). The relatively high value despite the lack of willingness and the relatively small distance to the "willing" shows that rather unmotivated employees can possibly achieve a direct benefit by concrete measures which promise a direct benefit.

\subsubsection{Nutrition consulting internal single}

So extremely positive is the age group 56-65 for the "healthy snacks", the rejection of this age group is so extreme for internal advice on weight reduction and nutritional change. With 4.1, none of the other measures in any age group is poorer. This may be since increasing weight and other health problems are not discussed with the physician in his/her own practice, as his role as an employer is generally viewed as weighing strengths and weaknesses. However, this null hypothesis

$H_{0}$ : The preference value of the age group 56 to 65 for the "Internal consulting" measure does not differ from the other age groups.

with $\mathrm{p}>5 \%$ is also statistically not significant, so that the null hypothesis can neither be rejected nor confirmed.

From the aspect of "experience" can the null hypothesis

$\mathrm{H}_{0}$ : The preference value for MFAs for the "Internal consulting" measure shows no difference between experienced and inexperienced general practitioners' practices

be rejected at a high level of significance with $\mathrm{p}<1 \%$. The counterhypothesis

$\mathrm{H}_{1}$ : The preference value for MFAs for the "Internal consulting" measure shows differences between experienced and inexperienced general practitioners' practices.

is hereby confirmed. The data analysis shows that inexperienced employees (3.5) clearly reject this offer rather than experienced (3.1).

As was to be expected, the preference of MFAs, which have indicated their general willingness to join health-promoting activities, tends to be better than that for the "unwilling" (average 3.2 resp.)

\subsubsection{External nutrition consulting single session}

As can be seen from the preference profile, the assessments of the individual groups do not significantly differ from each other.

Under the experience aspect, there is no significant ( $\mathrm{p}>5 \%$ ) difference in preference between experienced MFAs (average 2.8) and inexperienced (average 2.8).

As was to be expected, the preference of MFAs, which have marked their general willingness to join health management activities, tends to be better than that of the "unwilling" (average 2.4 resp. 3.4). The relatively great difference in the assessment suggests that there may be reservations about individual discussions, as health and 
nutritional habits are more likely to be attributed to privacy than to the operational sphere.

\subsubsection{External nutrition consulting group session}

As can be seen from the preference profile, the assessments of the individual age groups do not differ significantly.

In the "experience" aspect, there is no significant ( $>5 \%$ ) difference in the preference estimation between experienced MFAs (average 2.9) and inexperienced (average 3.2 ). Since the $\mathrm{p}$-value is $6 \%$, the counterhypothesis would be at a confidence level of $94 \%$ accepted.

$\mathrm{H}_{1}$ : The preference value for MFAs for the measure "external consulting group session" shows differences between experienced and inexperienced general practitioners' surgeries.

At the same time, inexperienced MFAs (3.2) tend to rate worse than experienced (2.9)

As was to be expected, the preference of MFAs, which have indicated their general willingness to participate in health-promoting measures, tends to be better than that for the "unwilling" (average 2.9 resp. 3.6).

\subsubsection{Preference profile to programs of vaccination}

Vaccination awareness in the general practitioners' surgeries is very high. $89 \%$ of MFAs regularly check their vaccination status. The vaccines in general are the best accepted activity with a ratification of $88 \%$ for the question "Would you join a vaccine program at work?" Regular vaccinations are made in the private life of $75 \%$ of MFAs. For detailed data see Table 21 to 24.

A very high percentage are already checking their vaccines status or use vaccines regularly. The high percentage shows the fundamental support for the vaccines in this employee's group.

As mentioned above the area of vaccines is discussed by some groups very emotional. This may explain the extreme difference. Based on the evaluation of the questionnaire the number of participants who use the open question was significantly lower than the number of answered multiple-choice questions. 


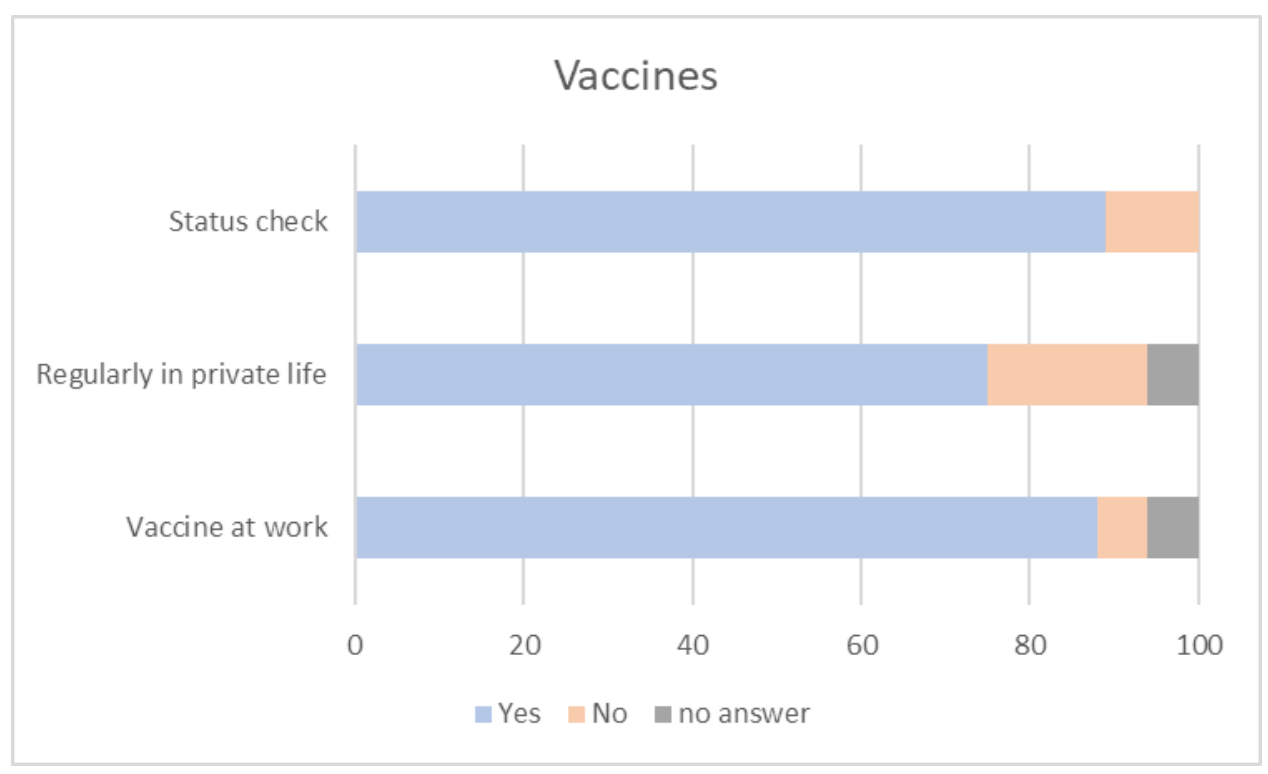

Figure 26: Vaccination status of the MFAs

There was no multiple-choice for the kind of vaccines. Every participant who wrote TBE vaccine or the flu vaccine needed to know that these vaccines are existing and remembered the name und wrote it down instead of making a tick. It is likely that this reduced the number of tick/comments. In the open question about six participants explicitly denied the flu vaccine, this would be a hint that especially this vaccine is not accepted and that it is not a problem of the questionnaire or its structure.

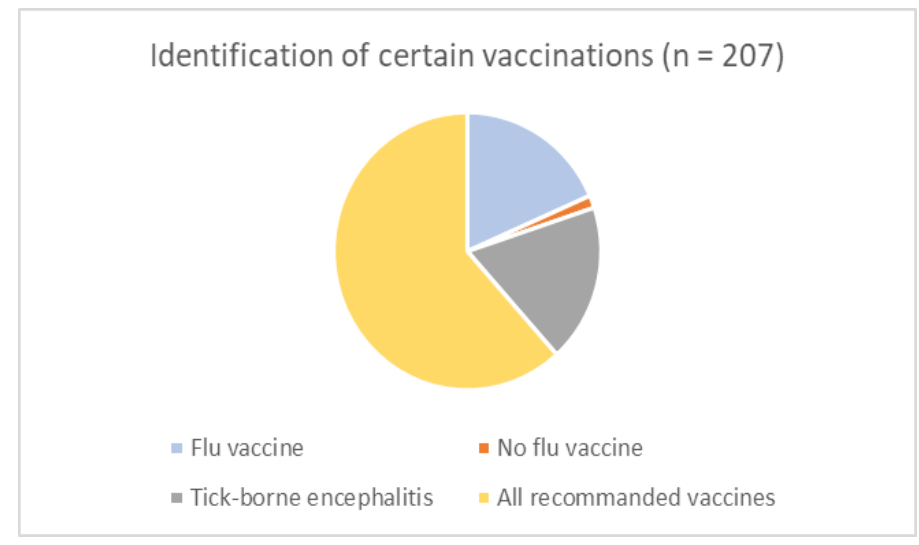

Figure 27: Kind of vaccines

For health management programs the high acceptance and usage can be an advantage because the acceptance of vaccines could help to raise the rates of flu vaccines which reduce the absence days (Institut für betriebswirtschaftliche Analysen, 2012) and improve the health management. In opposite to the activities for more movement and improved nutrition the vaccine could be a punctual action once a year.

The challenge could be that the flu vaccines are a yearly vaccine and the employer has to motivate the employees every year. The open questions were used by three participants to deny the use of flu vaccines. An effect of this could be that the acceptance of this special vaccine is low. However, following the literature review the 
flu vaccine is the most effective vaccine for reduction of absence days and the improvement of general health management. Another remarkable aspect is that the level of vaccines usage is very high in private life and at work. Nearly all participants who ticked "yes" for private ticked also "yes" for the support of vaccine campaign within the organisation.

The vaccine-related null hypotheses

$\mathrm{H}_{0}$ : The regular, up-to-date use of the vaccination offer by the MFAs is independent of age.

$\mathrm{H}_{0}$ : The willingness of the MFAs to be vaccinated by the employer is independent of age.

$\mathrm{H}_{0}$ : The regular check of the vaccination status of the MFAs is independent of age.

were tested for significance. None of the three null hypotheses could be rejected. It appears that age and vaccination behaviour are not associated with the age group of MFAs.

The same applies to the null hypotheses, which are based on experience with health management and general willingness to participate in activities. The vaccination behaviour seems to be independent of this.

This is different with the null hypotheses on the vaccine-related questions:

$H_{0}$ : The responses to regular vaccination and willingness to be vaccinated in practice are equally distributed.

$H_{0}$ : The responses to regular vaccination and the private verification of vaccination status are equally distributed.

$H_{0}$ : The responses to the willingness to be vaccinated in practice and the private verification of the vaccination status are equally distributed.

All null hypotheses can be statistically significantly rejected; it cannot be concluded from the fact that some MFAs will not be vaccinated in their own surgery that they can neither check their vaccination status nor can they be vaccinated regularly.

\subsubsection{Acceptance of further health management activities}

So far, the preferences on the nine "standard" measures have been examined. The aim of this subsection is to examine the replies to the open questions to examine whether further measures proposed by the MFAs should be included in addition to the measures proposed in the literature.

The general acceptance of health management is the basis to motivate employees to take part in health management activities. After the general acceptance, the acceptance of single activities is important. As mentioned above several participants 
did tick "no" at the question "Would you join a health management program". Afterwards they ticked several activities they would like. This could mean that an attractive health management activity could lead to employees to take part. This could motivate employees who do not "like" health management. An employer cannot force employees to take part in these activities, but the activities could be so attractive that they are used on a voluntary basis.

The results from the open question provide the activities, which were written down without any suggestion. It has the disadvantage that the number of comments is less compared to the number of ticks at the multiple-choice question.

The following table is based on Table 10 and summarizes the answers to the open questions supplemented by the percentages. In the questionnaire, the open question about which health management activities the participant would join was positioned before the multiple-choice question. This is relevant, if participants completed the questionnaire in a hurry, they would have written their prioritized activity in the open questions and afterwards marked it in the multiple-choice question.

There results from the open questions are partly coherent with the result from the multiple-choice questions. Therefore, nutritional, fitness and vaccine-related measures are not dealt here separately. The by the grey font marked activities are not included in the list of activities which are provided in the multiple choices question.

\begin{tabular}{|l|r|r|}
\hline $\begin{array}{l}\text { Are there activities for health management in your general practi- } \\
\text { tioner's surgery? If "yes" what kind of activity? }\end{array}$ & Total & Percentage \\
\hline & 12 & $30 \%$ \\
\hline Check up & 14 & $35 \%$ \\
\hline Vaccine & 5 & $12.5 \%$ \\
\hline Nutrition advice & 3 & $7.5 \%$ \\
\hline Healthy food & 1 & $2.5 \%$ \\
\hline Office chair for the back & 3 & $7.5 \%$ \\
\hline Fitness & 2 & $2 \%$ \\
\hline Vitamin infusion & 40 & \\
\hline Total & 40 \\
\hline
\end{tabular}

Table 28: Choice of activities in open questions

As described in subsection 5.2.2, check-ups are included in the conventional treatment programs and, as indicated in subsection 2.2.3, are not included in the primary prevention measures. They are part of the secondary prevention measures and should therefore not be included.

The "Office chair for the back" was only mentioned by one participant. Following the research "Effectiveness of participatory ergonomic interventions on health outcomes: a systematic review“ (Rivilis, van Eerd et al., 2008) single actions like buying ergonomic office chairs do not lead to an improved health management. A systematic method is needed. The ergonomic chairs are only mentioned by one person. That does not justify adoption of a general recommendation. 
Whether vitamin infusions can generally be recommended to a large audience, or whether possible side effects make this seem not advisable, is discussed in medical circles and non-medical circles. For this reason, and because here again the number of entries is far too small, the list of recommended measures has not been extended by this point.

Overall, the open questions did not lead to any broadening of the proposal list.

\subsubsection{Preference profiles to combined programs}

The nutrition could be combined with activities for movement. However, the activity programs need to be seen in a wider context including the general area of weight reduction. There are no studies, which confirm a direct link between physical activities and lower absent days. The link between movement and weight reduction shows the influence on the absent days. Movement has a positive influence on being overweight, which has an influence on the absent days (Laaksonen et al., 2009) but this connection is only indirect.

The study "How do physical activity, sports, and dietary restraint relate to overweight-associated absenteeism?" (van Strien and Koenders, 2010) endorse this link. Health management programs, which focus on promoting the physical activities, combined with other actions could reduce overweight of their employees. In this linkage, the packages of different activities in health management can be successful.

To find out which combinations of measures were acceptable or rejected, the database with the questionnaires was evaluated as follows:

All of the 5 movement-promoting measures were combined with the 4 foodpromoting measures. This results in a table with 20 entries containing all conceivable two combinations. A single table field contains a certain combination - e.g. "Training at home" and "Internal nutrition advice".

The database has been searched for entries that have a value of "1" or "2" on the Likert scale ("yes" or "rather yes"), both in "Column" activity and "Row" activity. This entry was then counted as "Yes".

Table 29 shows the number of approving entries per measure combination.

In order to obtain a clear overview, the denominations were converted into percentages and displayed as a bar chart (Figure 28).

The most popular combination of measures is therefore "Sponsored activities Healthy snacks at work" with a clear distance from all other combinations. The least popular is the combination "Training at workplace - internal single consulting". It is a clear sign that measures taking place in the own medical practice are not so popular. 


\begin{tabular}{|l|c|c|c|c|c|c|}
\hline \multicolumn{1}{|c|}{ Approbation } & $\begin{array}{c}\text { Training } \\
\text { at work- } \\
\text { place }\end{array}$ & $\begin{array}{c}\text { Training at } \\
\text { home }\end{array}$ & $\begin{array}{c}\text { Sponsored } \\
\text { activities }\end{array}$ & $\begin{array}{c}\text { Step coun- } \\
\text { ter }\end{array}$ & $\begin{array}{c}\text { Training } \\
\text { with col- } \\
\text { leagues }\end{array}$ & Sum \\
\hline $\begin{array}{l}\text { External group } \\
\text { consulting }\end{array}$ & 32 & 33 & 42 & 29 & 34 & 170 \\
\hline $\begin{array}{l}\text { External single } \\
\text { consulting }\end{array}$ & 38 & 47 & 57 & 48 & 37 & 227 \\
\hline $\begin{array}{l}\text { Internal single } \\
\text { consulting }\end{array}$ & 21 & 24 & 25 & 22 & 24 & 116 \\
\hline $\begin{array}{l}\text { Healthy snacks at } \\
\text { work }\end{array}$ & 69 & 77 & 98 & 74 & 65 & 383 \\
\hline \multicolumn{1}{|c|}{ Sum } & 160 & 181 & 222 & 173 & 160 & 896 \\
\hline
\end{tabular}

Table 29: Combination of measures with consent

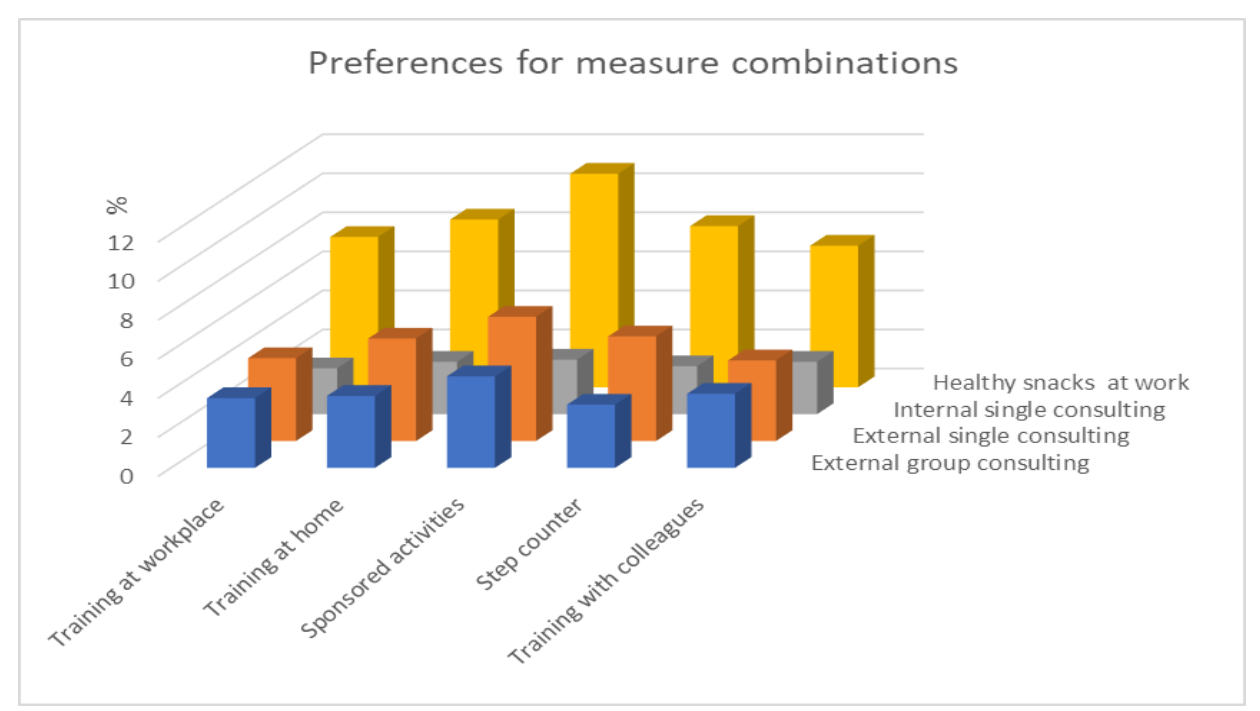

Figure 28: Approbation for measure combinations

If the "healthy snacks" measure is based on fundamental considerations, e.g. costs, not offered, the combination "Sponsored activities - External single consulting" is seen as the strongest preferred combination.

Furthermore, the ranking of a nutritional measure cannot be changed by the combination with a movement measure. For example, e.g. "External single consulting" (rank 2) is more popular than "External group consulting" (rank 3). No matter which movement measure "external group consulting" is combined, the rank does not change.

To check whether the refusal behaves in a mirror-like manner, the above-mentioned database query was carried out analogously with the values 4 and 5 on the Likert scale ("rather no" and "no"). The values converted into percentages are shown in the figure. 


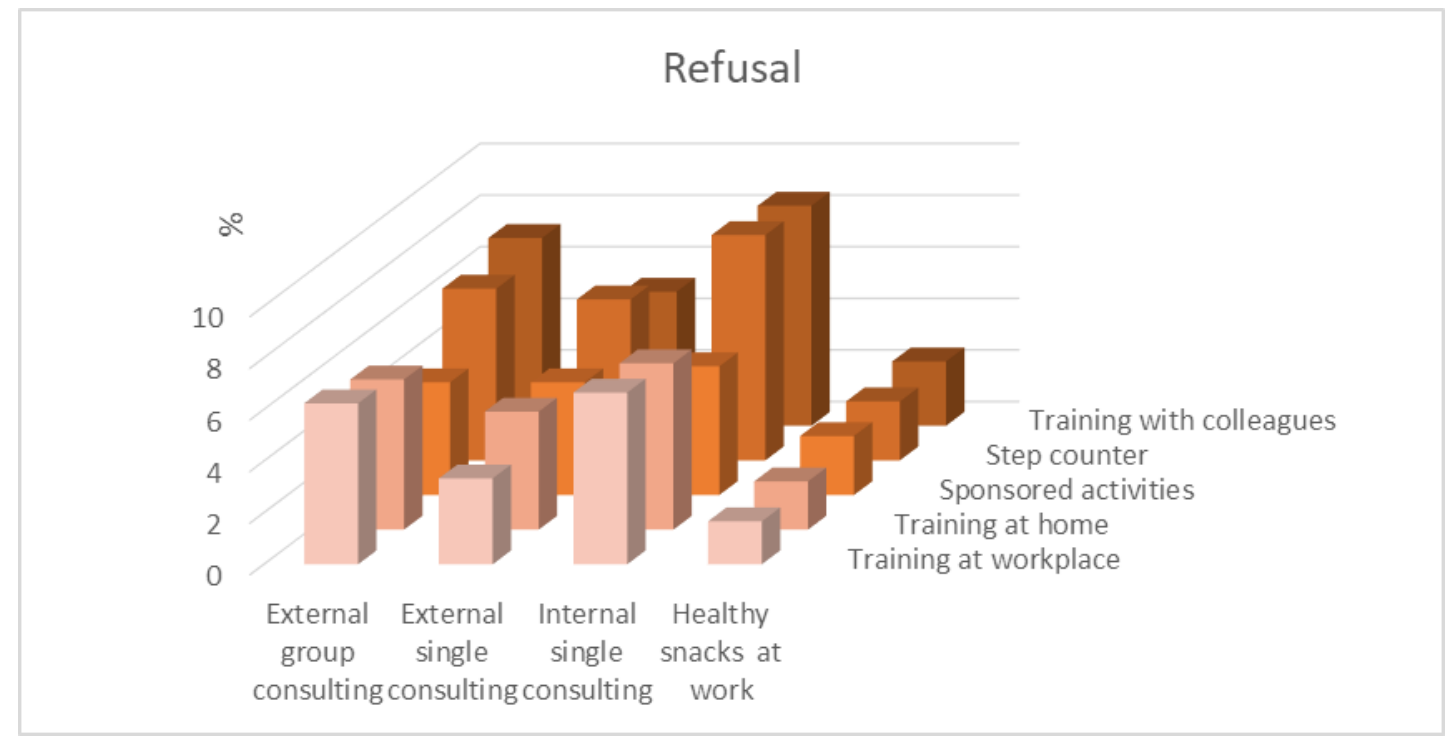

Figure 29: Rejection of action packages

In wide areas, refusal and consent are reflected in a mirror image. Thus, the combination with the lowest preference is also the combination with the strongest rejection. It is therefore recommended for a physician who wants to start a health program and plans to do so with internal counselling and training with colleagues has to realize resistance.

It is also clear from the "refusal" diagram that group-specific activities are more difficult compared to individual support. The combination of "external group nutrition consulting - training with colleagues" is one of the most unpopular.

Finally, the research examined the extent to which a program combination of the ten proposed measures can be found for each MFA which will be accepted. A program combination is a package of vaccination in the workplace, a movement program and a nutrition program. The database with all questionnaires were evaluated and classified each package as follows.

very suitable

The MFA has evaluated at least one of the exercise programs with 1 on the Likert scale ("Yes") and at least one of the nutrition programs with 1.

suitable

The MFA has assessed at least one of the exercise programs with 1 on the Likert scale and at least one of the nutrition programs with 2 . The vaccination program at the workplace is accepted.

or

The MFA has evaluated at least one of the exercise programs with 2 on the Likert scale and at least one of the nutrition programs with 1 . The vaccination program at the workplace is accepted. 
not suitable

The vaccination program at the workplace was not selected and all the no-two combinations were rated higher than 3 ("neutral").

All questionnaires $(\mathrm{N}=194)$ were evaluated with this classification. As the following figure shows, about $24 \%$ would find a "very suitable" health program in which they would participate. A further $66 \%$ would find a suitable offer. In sum, therefore, almost $90 \%$. For the remaining $10 \%$ it would be necessary to examine the obstacles to participation.

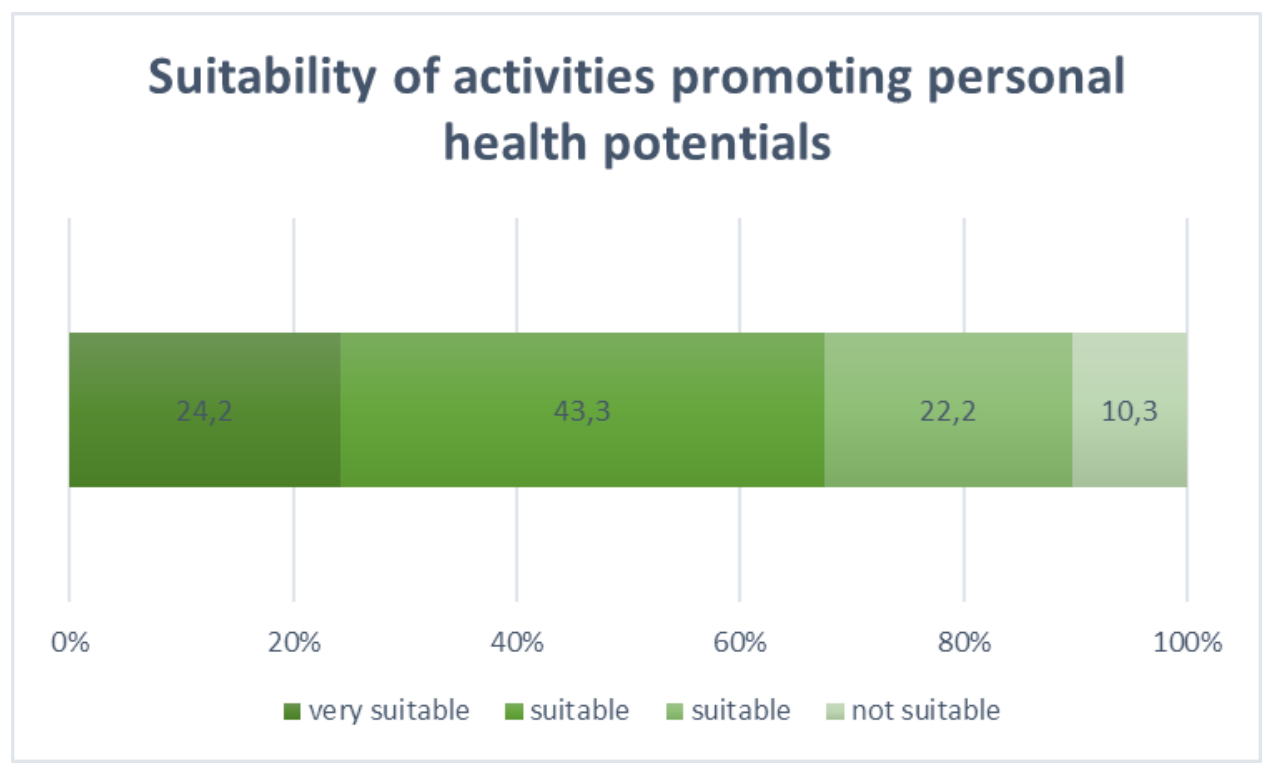

Figure 30: Suitability of combined programs

\subsection{Summary of results}

The following points summarize the research findings of the MFA Survey.

They contain the essential hypotheses and show the contribution to knowledge for the area "Preference profile of German MFAs in general practitioners' surgeries to activities promoting personal health potentials".

Moreover, the research question

2. Which of these measures (which indicate a strong link between measures of health promotion and a direct result in reduction of absenteeism) are already practised and which are preferred by the staff of the general practitioners' surgeries?

is answered:

- The MFA survey confirms that the MFA profession is a typical women's occupation, which is recognizable by the high proportion of women (98\%). 
- Compared to the working women the MFAs are significantly younger (36 years compared to 44 years). The age structure with two large age groups (18-25 years and 36-45 years) is atypical compared to the working women with the dominant age group 46 to 55 years.

- The percentage of general practitioners' surgeries with health-promoting activities is higher than that of SMEs. $26 \%$ of general practitioners practice health management activities. Vaccination programs with $35 \%$ are the top priority, followed by a healthy nutrition (20\%) and fitness ( $8 \%)$.

- With $63 \%$ the willingness to participate in health promotion measures is high. It is statistically significantly dependent on the age group. The group of middle-aged MFAs (36 to 45 years) shows the highest proportion of nonattendance both relatively and absolutely.

- The MFAs can be divided into four groups according to their willingness and experience. The group with "no experience" but "willingness to participate" has the largest share (51\%), whereas the group with "experience" but "not willing to participate" has the least share $(8 \%)$.

- The consent rates for the five proposed programs for the promotion of movement vary between $60 \%$ and $38 \%$. The most popular are "sponsored activities", the weakest is "training in a group with colleagues". In the overall picture the preferences for activities and the five age groups are independent of age. Statistically significant, the following peculiarities can be observed:

- "Training within a group with colleagues" is significantly less preferred by the age group 26-35 years than in the other age groups

- "Step counter and Fitness-Watch" are clearly not desired by the age group 56 to 65 years.

- "Training at workplace" is significantly more preferred by the age group 56 to 65 years than in the other age groups.

- Under the aspect of "experience", all measures of promotion of movement, apart from "sponsored activities", are more likely to be preferred by experienced MFAs than by inexperienced MFAs.

- The approval rates for the four proposed programs for weight reduction and nutrition change vary between $84 \%$ and 26\%. Most MFAs prefer "healthy snacks/food at work" most, and the "internal session with a physician from the medical office" weakest. In the overall picture, the preferences for all activities and the five age groups are independent of the age. Statistically significant, no peculiarities can be observed.

- From the point of view of "experience", the measure "internal session with a physician from the medical office" is more preferred by experienced MFAs rather than by inexperienced ones. At a slightly lower level of significance 
(6\%), this also applies to the "group meeting with a nutrition consultant" measure.

- As was to be expected, the preference of MFAs, which have marked their general willingness for health promotion measures, tends to be higher than that for the "unwilling" MFAs.

- The vaccination status is at an elevated level in general practitioners' surgeries. A vaccination program offered by the surgery finds the highest rate of consent at $88 \%$. Potential upside would still have the annual flu vaccination. The openness to vaccination is independent of age, previous experience and/or general willingness to participate in health management activities.

- The suggestions made by the MFAs on the measures of the primary prevention do not go beyond what is found in the literature.

- The most preferred combination with a movement and a nutritional program is "Sponsored activities (Fitness, Yoga...)" with "Healthy snacks/food at work". The least coveted combination of measures is "Training within a group of colleagues" with "Internal nutrition consulting with a physician from the medical office."

- In the combination of 1 vaccination, 5 movement and 4 nutrition programs, $90 \%$ find an offer to which they can say "yes". 10\% cannot find a vaccination, nutritional and movement offer, to which they clearly agree. 


\section{Research findings for the development of a priority concept}

The findings of the MFA survey, as presented in the previous chapter, are of crucial importance for the introduction of health management activities, as the acceptance and the resulting motivation are decisive for the success. Section 1 shows how these results are used for scoring. With effectivity and cost level two further stakeholder interests are added to the employee perspective.

Effectivity is the qualitative stakeholder perspective on the proposed measures. Since MFA primary prevention activities always involve a health aspect, the general practitioner should be included as specialists in his/her role as a physician. In section 2, besides the results of the literature review, the assessments of general practitioners are considered, as they result from the qualitative interviews.

The cost level represents the manager and owner's perspective on general practitioners' surgeries. The physician in his/her role as owner and manager is interested in economic success. The proposed measures may have been accepted by employees and tested for their effectiveness, but a balanced decision should include the cost perspective. This is the topic of section 3 .

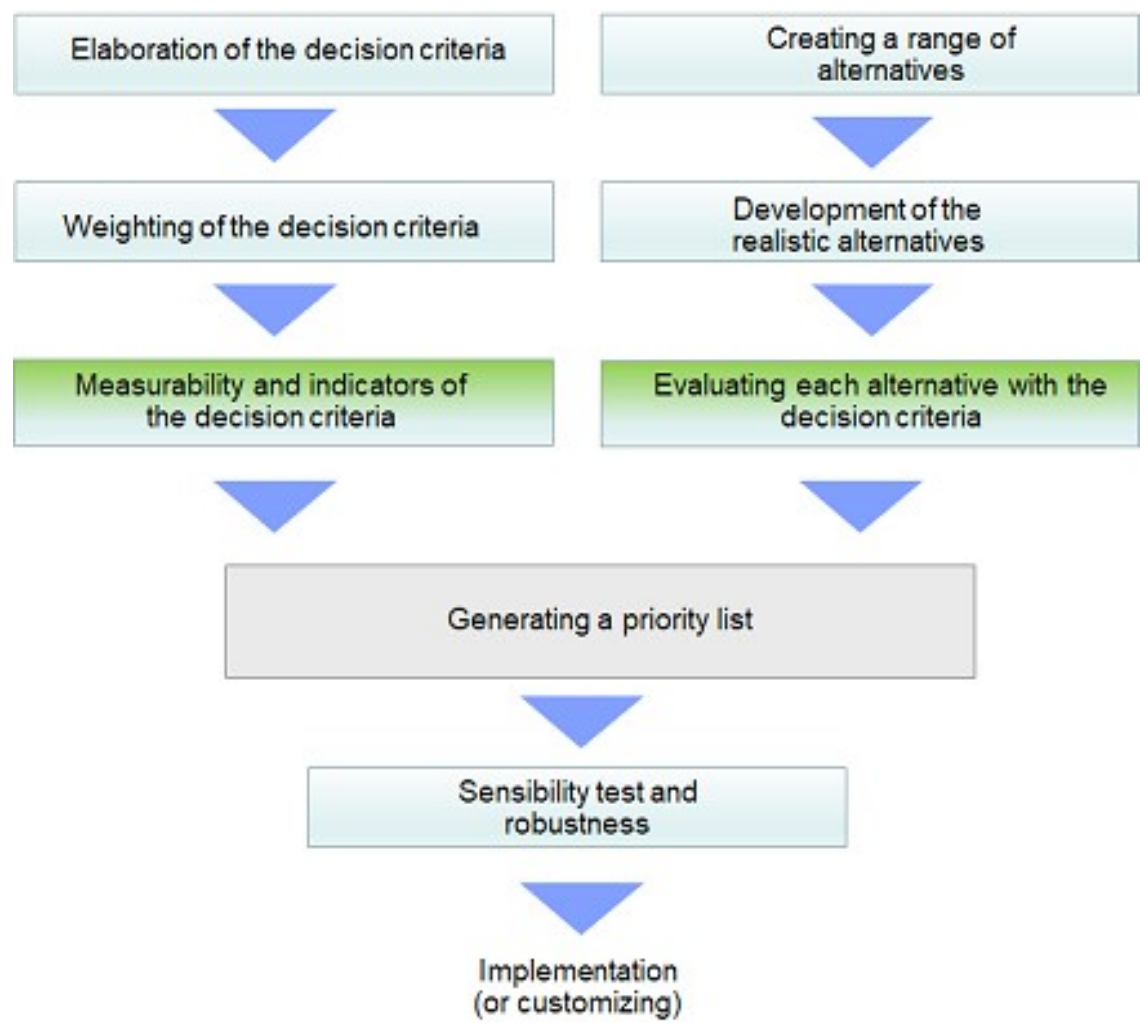

Figure 31: Multi-criteria decision-making model: Scoring the decision criteria

An assessment of the expected costs and an assessment of the effectiveness are therefore made for each of the ten recommended measures. With these inputs, the creation of a priority list is then made in section 4 in accordance with the multi-criteria deci- 
sion-making model presented in Figure 2. The priority list generated with the MMACBETH software (Bana e Costa, De Corte et al., 2014) shows a ranking of all measures considering all three decision criteria, thus presenting the most favourable activities. Moreover, comparative considerations are made in this section, if only one or two of the dimensions of the decision are put into focus. This may be important in the implementation.

A sensitivity analysis and a robustness check are carried out in section 5 with the aid of the M-MACBETH software (Bana e Costa, De Corte et al., 2014). This is done to check the order of the priorities for the case of changes and insecurities in weighting.

Subsection 4.3.7 sets out hypotheses that cover both the behaviour of MFAs in terms of other perspectives and the relationship of decision criteria among themselves. Section 6 tests these hypotheses and presents correlations.

For the prioritisation the criteria weighting, the acceptance score, the efficiency and the cost level score are needed. Each perspective has an influence on the priority depending on its weighting. The criteria weighting is central for the decision-making process. It defines the relevance or the importance of the degree of goal achievement. Each criterion and each activity are compared pairwise with each other. In this analyse three criteria are applied.

- Acceptance - weighting in relation $50 \%$

- Effectivity - weighting in relation $25 \%$

- Cost level - weighting in relation $25 \%$

Each criterion needs a weighting compared to the other. Together all three perspectives are weighted with $100 \%$ in sum.

\subsection{Scoring the acceptance}

The questionnaire gave the primary data for the acceptance. The percentage of acceptance is used as a score. As described in subsection 4.3.2 the score of 100 points which is the maximum on the positive side of the scale is identical with $100 \%$. Zero percent is the opposite with zero points as the negative side of the scale. Table 16 is used here as a template and Table 17 (activity promotion), Table 20 (weight reduction and change of nutrition), and Table 23 (vaccines) lead to the entries into the scoring table for acceptance. The original envisaged inclusion of further measures proposed by the MFAs of primary prevention did not occur after analysis of the qualitative responses (5.2.6). Similarly, the inclusion of private vaccination programs did not take place, since the proposals mentioned therein are all contained in the standard vaccinations customary in Germany, or in the proposal on "Vaccinating programs at workplace". The other measures proposed by the general practitioner in this section are a placeholder, which will only be used in subsection 7.3.5 "Adaptation to own ideas". 


\begin{tabular}{|l|c|}
\hline \multirow{2}{*}{ Personal health promotion activities } & Acceptance \\
\cline { 2 - 2 } & Score \\
\hline Programs for activity promotion & $45 \%$ \\
\hline - Training at the workplace & $50 \%$ \\
\hline - Training at home & $60 \%$ \\
\hline - Sponsored activity after work & $48 \%$ \\
\hline - Training with step counter or fitness watch & $39 \%$ \\
\hline - Training within a group of colleagues & $34 \%$ \\
\hline Activities for weight reduction and change of nutrition \\
\hline - Group session nutrition consulting (external) & $47 \%$ \\
\hline - Single session nutrition consulting (external) & $26 \%$ \\
\hline - Single session nutrition consulting (internal) & $84 \%$ \\
\hline - Healthy snacks/food at work & \\
\hline Vaccinating programs & $88 \%$ \\
\hline - Vaccinating programs at work & no entry \\
\hline - Vaccinating in private life & no entry \\
\hline Further proposed measures & dummy \\
\hline - Proposed in the MFA survey & \\
\hline - Proposed by the general practitioner &
\end{tabular}

Table 30: Scoring the acceptance of personal health promotion activities

The acceptance of the employees is a knock-out criterion. If the activity is not accepted by the employees, it could not be implemented. However, no activity was totally denied by the employees.

Before the further proceeding, it is to be examined whether a knock-out criterion is present under acceptance aspects, which would exclude one or more alternatives from further consideration. This would be, for example, if one of the measures is rejected by a majority.

For example, it would be possible to rule out a measure that would be rated as "rather no" or "no" by more than 50\%. "Single session nutrition consulting (internal)" would be a candidate who just misses the mark. There are just $50 \%$ of the negative rates and no more than $50 \%$. But it would not make sense to take it from the selection list, since the individual general practitioners would be in a position to promote acceptance among their own employees and thereby receive more approval.

\subsection{Scoring the effectivity}

After the score for the acceptance the score for the effectiveness is needed. This information concerning the acceptance of employees is combined with the score of effectiveness. For this score the relevant data and studies from the literature review are categorized and weighted. The weighting is provided by the studies which were identified by the author's research. The results from the literature provide activities with proven effect to the health of employees. For the analysis each activity got a score for effectiveness which was supplemented by a rating of the interviewed general practitioners. 
For example the „Workplace Physical Activity Interventions: a Systematic Review“ (Dugdill, Brettle et al., 2008) found out there is a moderate influence through activities at work and from the use of step counters' this weighting of his research generates 50 points for the training at workplace. The study „How do physical activity, sports, and dietary restraint relate to overweight-associated absenteeism?" (van Strien and Koenders, 2010) found evidence that sports leads to reduced overweight and in their study a reduction of the absence days could be measured. They did not analyse sports during the work time, because of that the proved efficiency for the training at the workplace is weighted as low by 25 points.

The following table includes the relevant studies for each activity and the level of influence each activity has. The influence is classified through the weighting the study provides for this activity

\begin{tabular}{|c|c|c|c|}
\hline Activity promotion & Studies & $\begin{array}{c}\text { Level of } \\
\text { influence }\end{array}$ & Score \\
\hline \multirow[t]{3}{*}{ Training at the workplace } & Dugdill et al., 2008 & Moderate & 50 \\
\hline & van Strien \& Koenders, 2010 & low & 25 \\
\hline & Abraham \& Graham-Rowe, 2009 & low & 25 \\
\hline \multicolumn{3}{|r|}{ Average } & 33 \\
\hline Training at home & van Strien \& Koenders, 2010 & Low & 25 \\
\hline \multicolumn{3}{|r|}{ Average } & 25 \\
\hline $\begin{array}{l}\text { Sponsored activity after } \\
\text { work }\end{array}$ & van Strien \& Koenders, 2010 & Low & 25 \\
\hline \multicolumn{3}{|r|}{ Average } & 25 \\
\hline \multirow{3}{*}{$\begin{array}{l}\text { Training with step coun- } \\
\text { ter or fitness watch }\end{array}$} & Dugdill et al., 2008 & strong & 75 \\
\hline & Abraham \& Graham-Rowe, 2009 & low & 25 \\
\hline & van Strien \& Koenders, 2010 & moderate & 50 \\
\hline \multicolumn{3}{|r|}{ Average } & 50 \\
\hline $\begin{array}{l}\text { Training within col- } \\
\text { leagues }\end{array}$ & Abraham \& Graham-Rowe, 2009 & Low & 25 \\
\hline \multicolumn{3}{|r|}{ Average } & 25 \\
\hline
\end{tabular}

Table 31: Effectiveness of activity programs

The next activities are about the nutrition and weight reduction activities. For these activities a score is needed, too. In the next paragraphs the weighting for the nutrition activities is described.

The journal article „Are worksite interventions effective in increasing physical activity?" (Abraham and Graham-Rowe, 2009) provide evidence that worksite interventions have a small positive effect. This effect was weighted for the scoring as low effect for all trainings except sports with a pedometer.

The study „How do physical activity, sports, and dietary restraint relate to overweight-associated absenteeism?" (van Strien and Koenders, 2010) the authors found evidence that reduced overweight leads to reduced absent days. They stated that consulting about dieting would help to reduce absent days. The research "Monitoring and Evaluation of Worksite Health Promotion Programs - Current state of knowledge and implications for practice" (Engbers, 2007) provides evidence that activities 
against overweight have a positive influence on the health management. It states that the overweight is an upcoming problem for the health management. There is no clear statement about how strong the effect is. The weighting in the table is low.

The research „A site-specific literature review of policy and environmental interventions that promote physical activity and nutrition for cardiovascular health: what works?" (Matson-Koffman, Brownstein et al., 2005) has a focus on the health improvement by political campaigns and in governmental organisations. There is also a part of the research about the positive influence of nutrition activities in organisations. The authors provided evidence that there is a strong positive influence from healthy food at work and moderate influence for consulting and information activities.

The journal article "Health-related behaviours and sickness absence from work"(Laaksonen et al., 2009) found strong evidence that overweight has a negative influence on the absent days. In this article the authors do not provide a suggestion for activities which are recommended or tested. Therefore, this article was not included in the table.

The weighting for the activities is summarized in the following table.

\begin{tabular}{|c|c|c|c|}
\hline Nutrition & Studies & $\begin{array}{c}\text { Level of } \\
\text { influence }\end{array}$ & Score \\
\hline \multirow[t]{3}{*}{$\begin{array}{l}\text { Group nutrition consulting } \\
\text { (external) }\end{array}$} & $\begin{array}{l}\text { van Strien \& Koenders, } \\
2010\end{array}$ & moderate & 50 \\
\hline & Engbers, 2007 & low & 25 \\
\hline & $\begin{array}{l}\text { Matson-Koffman et al., } \\
2005\end{array}$ & moderate & 50 \\
\hline \multirow{4}{*}{$\begin{array}{l}\text { Single nutrition consulting } \\
\text { (external) }\end{array}$} & & Average & 42 \\
\hline & $\begin{array}{l}\text { van Strien \& Koenders, } \\
2010\end{array}$ & moderate & 50 \\
\hline & Engbers, 2007 & low & 25 \\
\hline & $\begin{array}{l}\text { Matson-Koffman et al., } \\
2005\end{array}$ & moderate & 50 \\
\hline \multirow{4}{*}{$\begin{array}{l}\text { Nutrition consulting by medical } \\
\text { doctor from the medical office }\end{array}$} & & Average & 42 \\
\hline & $\begin{array}{l}\text { van Strien \& Koenders, } \\
2010\end{array}$ & moderate & 50 \\
\hline & Engbers, 2007 & low & 25 \\
\hline & $\begin{array}{l}\text { Matson-Koffman et al., } \\
2005\end{array}$ & moderate & 50 \\
\hline \multirow{4}{*}{ Healthy snacks/food at work } & & Average & 36 \\
\hline & $\begin{array}{l}\text { van Strien \& Koenders, } \\
2010\end{array}$ & moderate & 50 \\
\hline & Engbers, 2007 & low & 25 \\
\hline & $\begin{array}{l}\text { Matson-Koffman et al., } \\
2005\end{array}$ & strong & 75 \\
\hline \multicolumn{3}{|c|}{ Average } & 50 \\
\hline
\end{tabular}

Table 32: Effectiveness score of nutrition programs

The last part of the questionnaire was about the vaccines. There was robust evidence for the use of vaccines during the literature review. The research „Occupational vac- 
cination of health care workers: Uptake, attitudes and potential solutions" (Little, Goodrigde et al., 2015) and the research „Healthcare workers and immunity to infectious diseases" (Vagholkar, $\mathrm{Ng}$ et al., 2008) both provided evidence that vaccine have a very strong and positive influence on the absent days. Both do not separate employees in hospital and general practitioners' surgeries.

\begin{tabular}{|l|l|l|c|}
\hline Vaccine & Studies & $\begin{array}{c}\text { Level of } \\
\text { influence }\end{array}$ & Score \\
\hline \multicolumn{3}{|l|}{} & \\
\hline Vaccinating program at work & Little et al. (2015) & strong & 100 \\
\hline \multicolumn{2}{|c|}{} & Strong & 100 \\
\hline \multicolumn{2}{|c|}{ Average } & 100 \\
\hline
\end{tabular}

Table 33: Effectiveness score for vaccine program activities

With the final scoring for the vaccines the weighting for the effectivity of the identified activities based on the literature review is completed.

The following table summarizes all the above results. In the column marked in yellow, the measures of the proposed standard measure are shown, the entries for the blue-marked effectivity determinations have been taken from the above tables.

\begin{tabular}{|l|c|c|}
\hline \multirow{2}{*}{$\begin{array}{l}\text { Personal health } \\
\text { promotion activities }\end{array}$} & $\begin{array}{c}|c| \\
\text { Stu- } \\
\text { dies }\end{array}$ & Score \\
\hline Programs for activity promotion & & \\
\hline - Training at the workplace & 3 & $33 \%$ \\
\hline - Training at home & 1 & $25 \%$ \\
\hline - Sponsored activity after work & 1 & $25 \%$ \\
\hline - Training with step counter or watch & 3 & $50 \%$ \\
\hline $\begin{array}{l}\text { - Training within a } \\
\text { group of colleagues }\end{array}$ & 1 & $25 \%$ \\
\hline $\begin{array}{l}\text { Activities for weight reduction and } \\
\text { change of nutrition }\end{array}$ & & \\
\hline $\begin{array}{l}\text { - Group session nutrition } \\
\text { consulting (external) }\end{array}$ & 3 & $42 \%$ \\
\hline $\begin{array}{l}\text { - Single session nutrition } \\
\text { consulting (external) }\end{array}$ & 3 & $42 \%$ \\
\hline $\begin{array}{l}\text { - Single session nutrition } \\
\text { consulting (internal) }\end{array}$ & 3 & $36 \%$ \\
\hline - Healthy snacks/food at work & 3 & $50 \%$ \\
\hline Vaccinating programs & 2 & $100 \%$ \\
\hline - Vaccinating programs at work &
\end{tabular}

Table 34: Score of the effectivity of personal health promotion activities

According to the evaluation of studies, vaccination at the workplace (100\%), healthy snacks or food at the workplace $(50 \%)$ as well as the training with step counter or fitness-watch $(50 \%)$ are the most effective measures from the point of view of reducing absenteeism.

As with the decision criterion acceptance it is to be examined whether a knock-out criterion is present under effectivity aspects, which would exclude one or more alternatives from further consideration. This would be, for example, if one of the measures has only a very poor indication of effectivity. 
For the studies, this can be excluded by the procedure in the literature review, since only studies which have an effect of the measures on the absence are expressly taken.

\subsection{Scoring the cost level}

The score for acceptance and efficiency are defined. The last missing perspective is the cost factor.

Following the methodology presented in subsection 4.3.4, the score for the cost level is calculated. The scoring is based on figures and the qualitative result of moderate, no or strong effect is transferred into a ratio

High cost or high time consuming 0

Medium cost or medium time consuming $\quad 50$

No cost or not time consuming $\quad 100$

The cost types to consider are:

Number of internal employees' hours

Number of internal leader (doctoral) hours

Number of external trainer hours

Estimated external costs in Euro

As described in subsection 4.3.4, the application of the multi-criteria decisionmaking software M-Macbeth (Bana e Costa et al., 2014) is not so much the absolute cost, but the cost relation. So, it is enough to note that "Sponsored activity" causes higher external costs than "training within a group of colleagues". Each position gets a weighting for the estimated costs for working hours and a weighting for the estimated costs.

From the aspect of working hours, the three advisory activities are at the highest level. The second highest category after working hours is the "training within a group of colleagues". This is not the cost of the consultant or the physician themselves, but the employees who participate in the training. Since several employees are participating at the same time and the organization of groups requires a lot of organizational effort and time, relatively high costs are incurred, even if the hourly rate of the MFAs is not too high. The concept "training at the workplace" assumes that, such as 10 minutes of exercise at the workplace, so it costs working time, but there are no side-effects. Therefore, the concept is rated "low". All other activities are subject to so few efforts that they can be rated as "none" in the overall comparison.

\begin{tabular}{|l|l|l|l|l|}
\hline Options & $\begin{array}{c}\text { Cost of work- } \\
\text { ing hours }\end{array}$ & Score & Cost & Score \\
\hline Training & Low & 75 & Low & 75 \\
\hline Training at the workplace & None & 100 & None & 100 \\
\hline Training at home & None & 100 & Medium & 50 \\
\hline Sponsored activity after work & None & 100 & Medium & 50 \\
\hline Training with step counter & Medium & 50 & Low & 75 \\
\hline Training within colleagues & & & & \\
\hline
\end{tabular}




\begin{tabular}{|l|l|l|l|l|}
\hline Options & $\begin{array}{c}\text { Cost of work- } \\
\text { ing hours }\end{array}$ & Score & Cost & Score \\
\hline Nutrition & & & & \\
\hline Group nutrition consulting (external) & High & 0 & Medium & 50 \\
\hline Single nutrition consulting (external) & High & 0 & Medium & 50 \\
\hline $\begin{array}{l}\text { Nutrition consulting by medical doctor from } \\
\text { the medical office (internal) }\end{array}$ & High & 0 & Low & 75 \\
\hline Snacks/food at work & None & 100 & High & 0 \\
\hline Vaccines & & & & \\
\hline Vaccinating program at work & None & 100 & None & 100 \\
\hline Vaccinating at home & None & 100 & None & 100 \\
\hline
\end{tabular}

Table 35: Scoring the cost level

The "Healthy Snacks/Food at Work" are to be assessed at the highest level in terms of external fix or acquisition costs. No matter how the concept is organized, there are daily costs that are attributable to this measure. Less costs, but still relatively high, are required if external consulting is necessary for nutritional advice. For the sponsored activities, which are on the same level "medium", the external costs are fees for fitness studios, yoga classes etc. Similar measures are to be evaluated here for "Step counter and fitness watch" as they have some extent of equipment, although developments like mobile apps can be expected to reduce costs. At a low-cost level, activities are concentrated in the workplace, as this requires a somewhat higher demand for space and, in the case of internal consultation, the possible takeover or participation in course fees for advisory recommendations. The costs for the vaccines are not relevant because the standard vaccines are all paid by the health insurances. If the physicians vaccinate their employees, they will be paid like vaccinating a normal patient. The employees do not have to pay for it.

\begin{tabular}{|l|c|}
\hline \multirow{2}{*}{ Personal health promotion activities } & $\begin{array}{c}\text { Cost level } \\
\text { Total Score }\end{array}$ \\
\hline Programs for activity promotion & $75 \%$ \\
\hline - Training at the workplace & $100 \%$ \\
\hline - Training at home & $75 \%$ \\
\hline - Sponsored activity after work & $75 \%$ \\
\hline - Training with step counter or fitness watch & $62 \%$ \\
\hline - Training within a group of colleagues & $25 \%$ \\
\hline Activities for weight reduction and change of nutrition \\
\hline - Group session nutrition consulting (external) & $25 \%$ \\
\hline - Single session nutrition consulting (external) & $37 \%$ \\
\hline - Single session nutrition consulting (internal) & $50 \%$ \\
\hline - Healthy snacks/food at work & $100 \%$ \\
\hline Vaccinating programs & no entry \\
\hline - Vaccinating programs at work & no entry \\
\hline - Vaccinating in private life & dummy \\
\hline Further proposed measures & \\
\hline - Proposed in the MFA survey & \\
\hline - Proposed by the general practitioner &
\end{tabular}

Table 36: Building the score of the costs of personal health promotion activities 
The following table summarizes all the above results. In the column marked in yellow, the measures of the proposed standard measures are shown, the entries for the blue-marked cost level determinations have been taken from the above table.

\subsection{Generating a priority list}

The section above provides all generated data in a score system. The next step is the transfer of these data into the M-MACBETH software (Bana e Costa et al., 2014) to analyse them.

The tables 30, 34, and 36 with the scores for each perspective was used as data basis for the following transfer into the software. The transfer started with the three criteria and the weighting of each criterion. The software provides the possibly to make a consistency check for this weighting. This test was made, and the weightings are consistent. This is important because inconsistent data could not be analysed with the software. In the next step all the defined activities were put into the options list.

For a better overview each activity (option) group has the same colour. This has no influence on the results it is just made to keep it more optical clear. Each of the three groups has its own colour. The nutrition activities are blue, the activity promotion options are pink, and the vaccine option is green.

\begin{tabular}{|c|c|c|}
\hline \multicolumn{2}{|l|}{ Options } & $x$ \\
\hline$-\quad+$ & Name & Short name \\
\hline 1 & Training at the workplace & Training at work \\
\hline 2 & Training at home & Training at home \\
\hline 3 & Sponsored activity after work & Sponsored activity \\
\hline 4 & Training with step counter & Step counter \\
\hline 5 & Training within colleagues & Training colleagues \\
\hline 6 & Group nutrition consulting (external] & Group nutrition ext. \\
\hline 7 & Single nutrition consulting (external) & Single nutrition ext \\
\hline 8 & Nutrition consulting by medical doctor from the medical office & Nutrition intern \\
\hline 9 & Healthy food at work & Healthy food at work \\
\hline 10 & Vaccinating program at work & Vaccinating at work \\
\hline & Properties & Performances \\
\hline
\end{tabular}

Figure 32: Options and activities list (screenshot)

The ten options were listed, and the score had to be transferred. Every element got its performance from the main score table. The figure shows e.g. the performance of "Training at work" on "Acceptance".

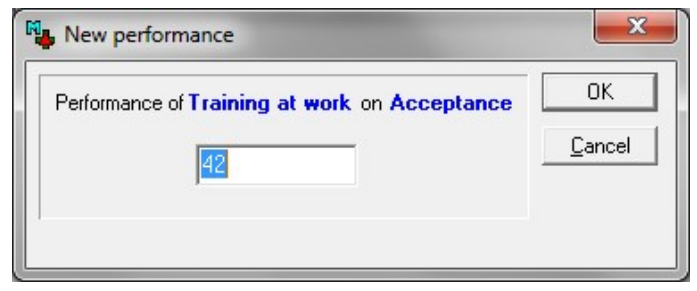

Figure 33: Score as performance indicator for a certain option/activity and decision criterion (screenshot) 
The final table was completed with all scores.

\begin{tabular}{|c|c|c|c|}
\hline \multicolumn{3}{|c|}{ 금 Table of performances } & $\mathrm{X}$ \\
\hline Options & Acceptance & Costs & Efficiency \\
\hline Training at work & 42 & 75 & 33 \\
\hline Training at home & 46 & 100 & 50 \\
\hline Sponsored activity & 57 & 75 & 50 \\
\hline Step counter & 45 & 75 & 66 \\
\hline Training colleagues & 37 & 62 & 16 \\
\hline Group nutrition ext. & 31 & 25 & 42 \\
\hline Single nutrition ext & 43 & 25 & 42 \\
\hline Nutrition intern & 31 & 37 & 42 \\
\hline Healthy food at work & 79 & 50 & 58 \\
\hline Vaccinating at work & 88 & 100 & 100 \\
\hline
\end{tabular}

Figure 34: Table of performance (screenshot)

This was the last step which was needed to start the analysis. All scores can be used for the calculation. The final priority list can be calculated by one click on. The software calculates the "Overall performance" in relation to all weightings and scores. The yellow highlighted column "Overall" of the "Table of scores" is ordered from the option with the highest performance to the lowest. Together with the column "Options" the both columns form the "Priority list".

The other columns contain the decision criteria. In each line, the performance on the individual decision criteria is displayed as well as the overall performance. The overall performance results as the weighted average of the individual performances. The weights used here are in the last line.

\begin{tabular}{|c|c|c|c|c|}
\hline \multicolumn{4}{|l|}{ Wh Table of scores } & $x$ \\
\hline Options & Overall & Acceptance & Costs & Efficiency \\
\hline [ all upper ] & 100.00 & 100.00 & 100.00 & 100.00 \\
\hline Vaccinating at work & 94.00 & 88.00 & 100.00 & 100.00 \\
\hline Healthy food at work & 66.50 & 79.00 & 50.00 & 58.00 \\
\hline Training at home & 60.50 & 46.00 & 100.00 & 50.00 \\
\hline Sponsored activity & 59.75 & 57.00 & 75.00 & 50.00 \\
\hline Step counter & 57.75 & 45.00 & 75.00 & 66.00 \\
\hline Training at work & 48.00 & 42.00 & 75.00 & 33.00 \\
\hline Single nutrition ext & 38.25 & 43.00 & 25.00 & 42.00 \\
\hline Training colleagues & 38.00 & 37.00 & 62.00 & 16.00 \\
\hline Nutrition intern & 35.25 & 31.00 & 37.00 & 42.00 \\
\hline Group nutrition ext. & 32.25 & 31.00 & 25.00 & 42.00 \\
\hline [ all lower ] & 0.00 & 0.00 & 0.00 & 0.00 \\
\hline \multicolumn{2}{|l|}{ Weights: } & 0.5000 & 0.2500 & 0.2500 \\
\hline
\end{tabular}

Figure 35: Priority list (screenshot)

With an overall performance of $94 \%$, vaccination programs are clearly ranked 1 ; they are therefore to be realized with highest priority. This final list could directly be used by general practitioners' surgeries to improve their health management. This will be discussed in the next section.

The vaccines have the best score in every criterion group. It does not matter how the criterion are weighted between each other, if the option is on the first place at all cat- 
egorise it will be on first place at the final ranking. The very high acceptance combined with no cost and strongly proved efficiency seems invincible. The distance between the vaccines with a score of 94 to the second place "healthy food at work" with a score of 66.5 is acceptable for this research. Compared to all other activities this is the widest distance. All other options are closer to each other.

The healthy food at the second position is a surprise. The rating for the costs is high but the employees prefer this option. The general practitioner needs to decide if this option is possible or if the costs are too high. A supported program for training at home could be a cheaper alternative.

Nearly with the same score, the financial supported activity at home has the next position. Generally, the movement promotion options are close together. The training with colleagues is below the other activity promotion options. The block of worst rated options is the area of nutrition consulting. They are all positioned in the last third of the list. However, these activities are still activities with a proven effect, and it is much better to implement these activities than activities with no effect. All these options can be used for the improvement of health management.

To be able to better compare the scores of the options in the individual decisionmaking criteria, the above list of priorities was presented with the software MS-Excel in a three-dimensional decision space.

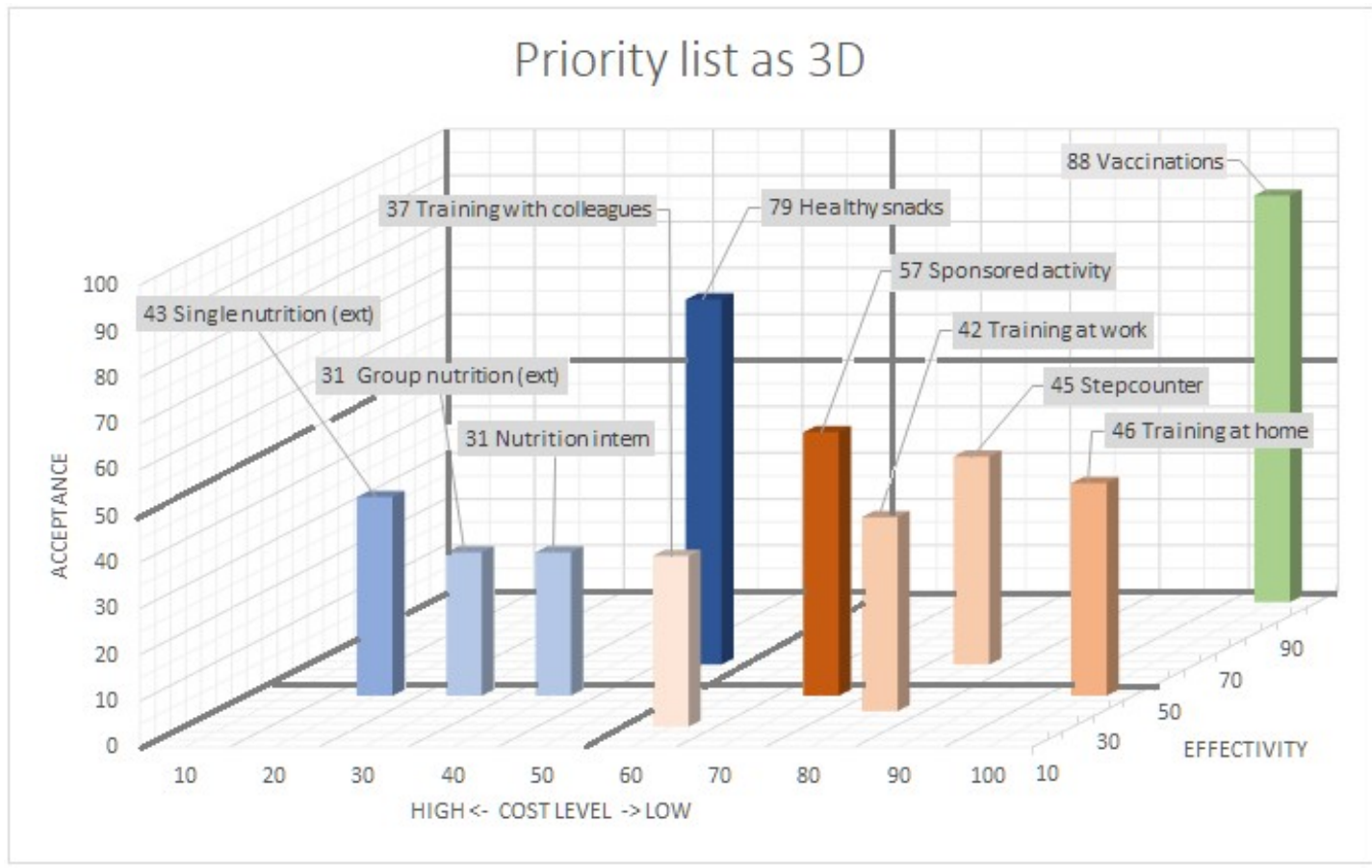

Figure 36: Priority list in a 3D-overview

On the $\mathrm{x}$-axis, the overall performance is entered for the decision criterion "Cost level". The higher the performance, the lower the cost level. The y-axis, which runs from the front to the rear in the depth, contains the performance for the criterion of "Effectivity". The up-going z-axis is provided for the scoring of the "Acceptance". 
For each option, the three values for cost (x), effectivity (y), and acceptance (z) show a unique point that indicates how the option is relative to other options. For each option, a column is entered at the respective point. The height of the column corresponds to their acceptance score. Thus, the column for vaccinations, coloured green as above, stands at the rear right to 100 (most favourable cost level) and 100 (highest efficiency). The height of the column is 88 , which corresponds to the entered acceptance value.

If this measure has already been implemented, it is interesting to know what the next optimal areas are. If two decision dimensions are combined, three areas and the associated basic attitudes are distinguishable:

- High acceptance and high effectivity, but also high costs (x-axis left, y-axis rear, z-axis fig. 41): this category includes the "Healthy Snacks/food at work". The decision for this measure is in line with the basic attitude: "The main thing is that something comes out of it and it is accepted, whatever the cost." (Overall performance: 66.5\%)

- Low cost and high acceptance, but low effectivity (x-axis right, $\mathrm{y}$-axis front, $\mathrm{z}$ axis top fig. 41): this category includes "Sponsored activity". The decision for this measure corresponds to the basic attitude: "The main thing, it comes with the employees and costs little." (Overall performance: 59.8\%)

- High effectivity and low cost, but low acceptance (x-axis right, y-axis rear, zaxis bottom fig. 41): this category includes "Training with step counter/fitness watch". The decision for this measure corresponds to the basic attitude: "The main thing, it comes out something and it does not cost much, I will motivate the staff for it." (Overall performance: $57.8 \%$ )

If one adds the "golden middle ground", the most important action strategies for implementation are recognizable.

- Medium acceptance, medium effectivity and low cost (x-axis right, y-axis centered, z-axis center fig. 38) - This category includes "Training at home". (Overall performance: $60.5 \%$ )

\subsection{Sensitivity and robustness test}

In this section, the possibilities of the software analysis are used, and the weighting of the criteria is changed to simulate different result and to learn more about the robustness and change of the priority list if the data input is different.

\subsubsection{Sensitivity versus changes in the acceptance weighting}

A first variation shows what would change in the "priority performance", if the weighting of the acceptance would change. Currently, the acceptance is $50 \%$ 
weighted. In the following M-MACBETH screenshot, this is recognizable by the vertical red line intersecting the $\mathrm{x}$-axis (acceptance) at $50 \%$.

The intersections of the red line with the lines for the individual measures show the percentage of the overall score in the value on the y-axis. The red line cuts, for example, the green line for "vaccinating at work" is $94 \%$. The intersection with the blue line for "Healthy food at work" is around $67 \%$. The purple lines for "Sponsored activity" and "training at home" are around $60 \%$. The current overall scoring can be read off the red line.

In the current scoring (Figure 35) "Training at home" is slightly above the "Sponsored activity". If the red weighting line, e.g. is shifted right to $70 \%$, the priority changes, "Sponsored activity" reach a higher overall score than "Training at home". At the same time, it becomes clear that a higher weighting of "Acceptance" does not lead to a change in the order of "Healthy food at work" and "Vaccinating at work".

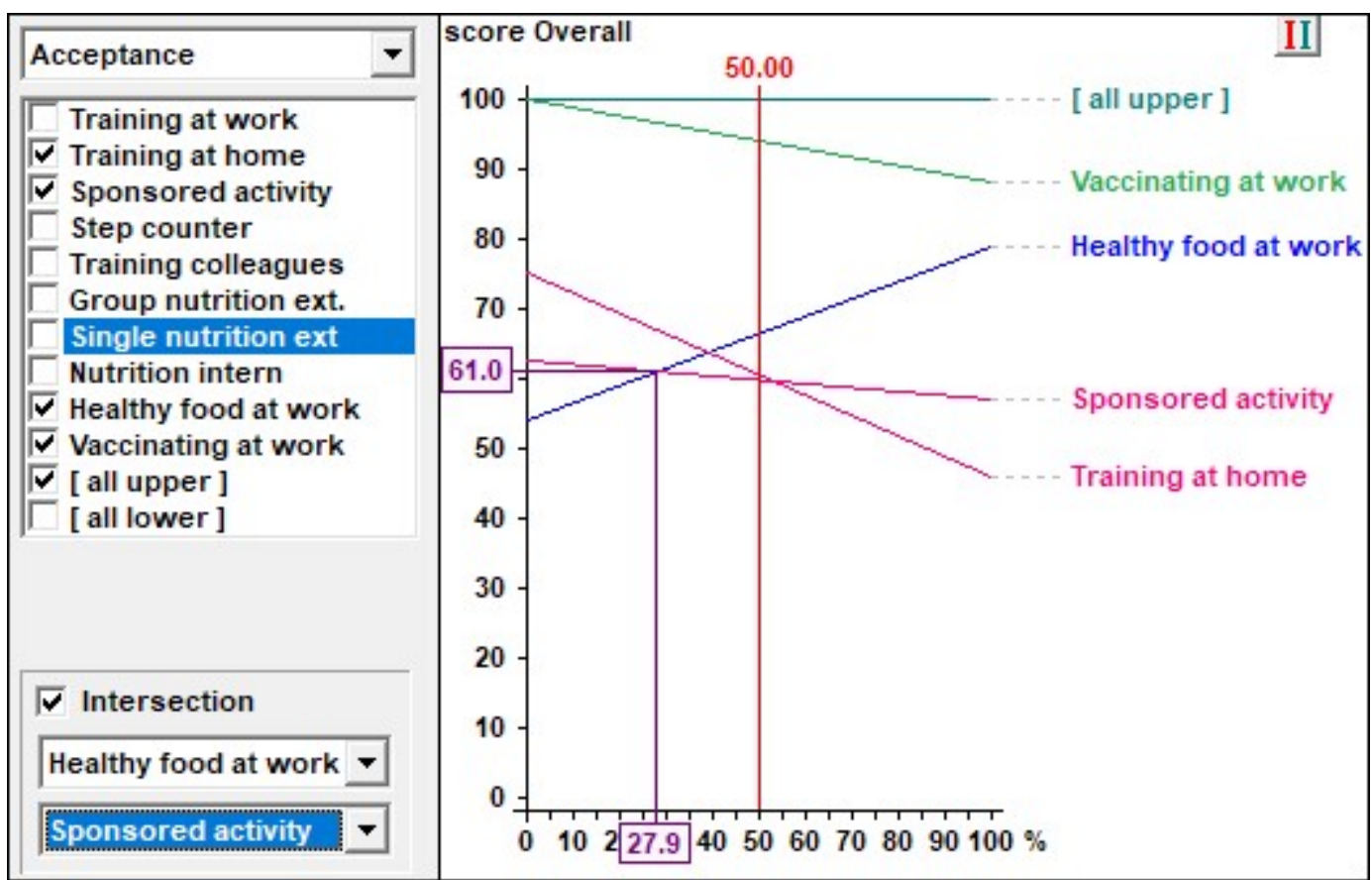

Figure 37: Sensitivity and robustness check for "Acceptance"

The intersection point $(27.9 ; 61.0)$ shown in the diagram indicates the point from which "Healthy food at work" is ranked higher than "Sponsored activity", when the acceptance is more than $28 \%$ in the total weight or otherwise spoken: Even if all three criteria were balanced $(33.3 \%)$, the priorities list would not change. 


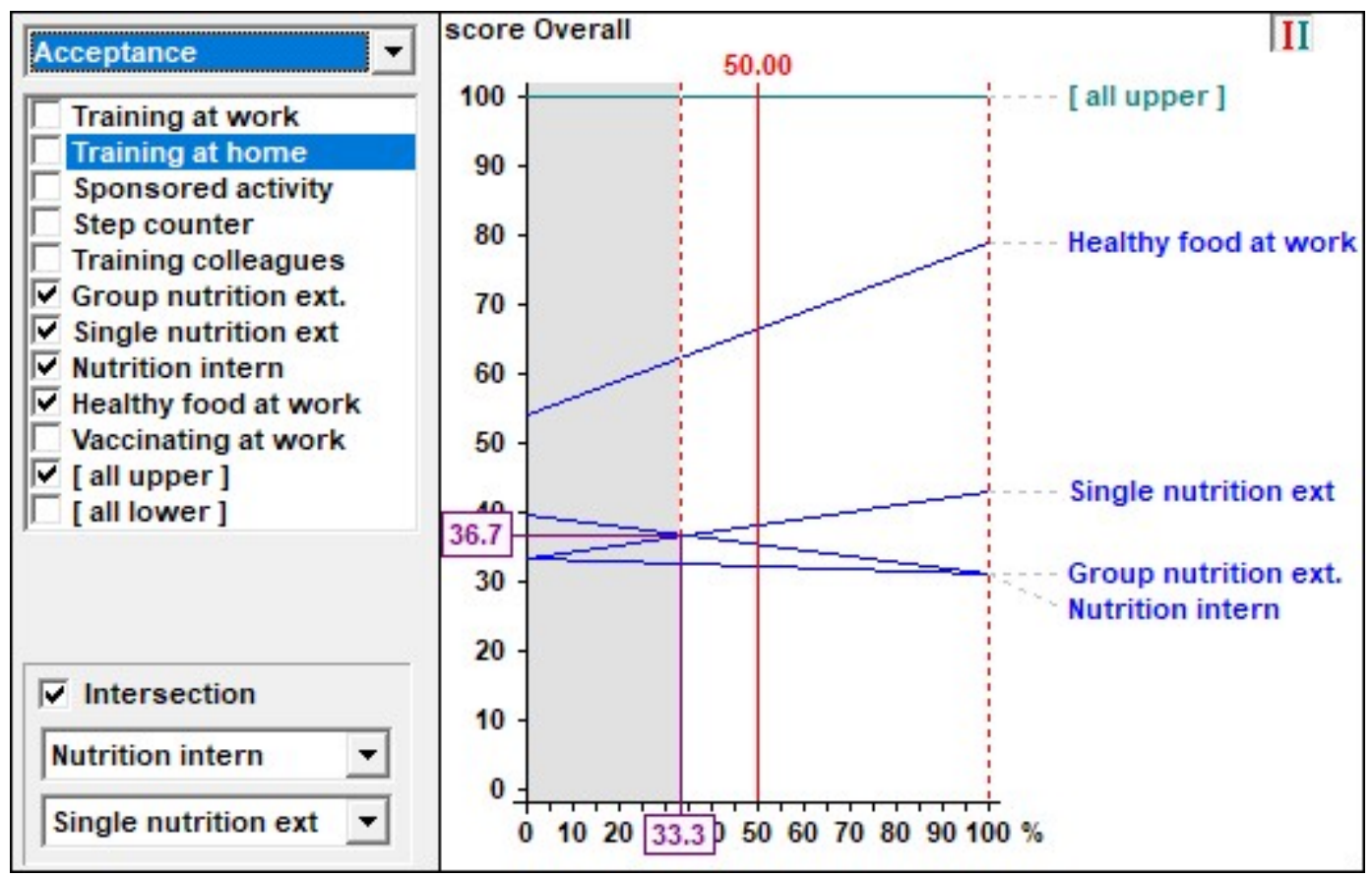

Figure 38: Sensitivity check for "Acceptance" within "Nutrition"

If one examines the sensitivity and robustness to fluctuations in the weight of acceptance only within the group "nutrition", there is also a great stability in the priority sequence. Only if the weighting was only one third, "Single nutrition ext." would be pushed before "Nutrition intern".

A similar picture emerges if you examine the group "Training" for sensitivity and robustness. Only if the acceptance is weighted with less than $40 \%$, "Sponsored activity" is displaced from the first place within the group.

\subsubsection{Sensitivity versus changes in the effectivity weighting}

The effectiveness is balanced with the cost level. They account for $25 \%$. As shown in the following figure, changing the weighting of effectiveness cannot change the rank of "Vaccinating at work". Starting with a weighting of $36 \%$, the "Step counter" measure becomes the highest priority measure within the training measures. From an (unrealistic) weighting of around $70 \%$, this measure rises to second place and is rated higher in the overall score than the nutritional measure "Healthy food at work". 


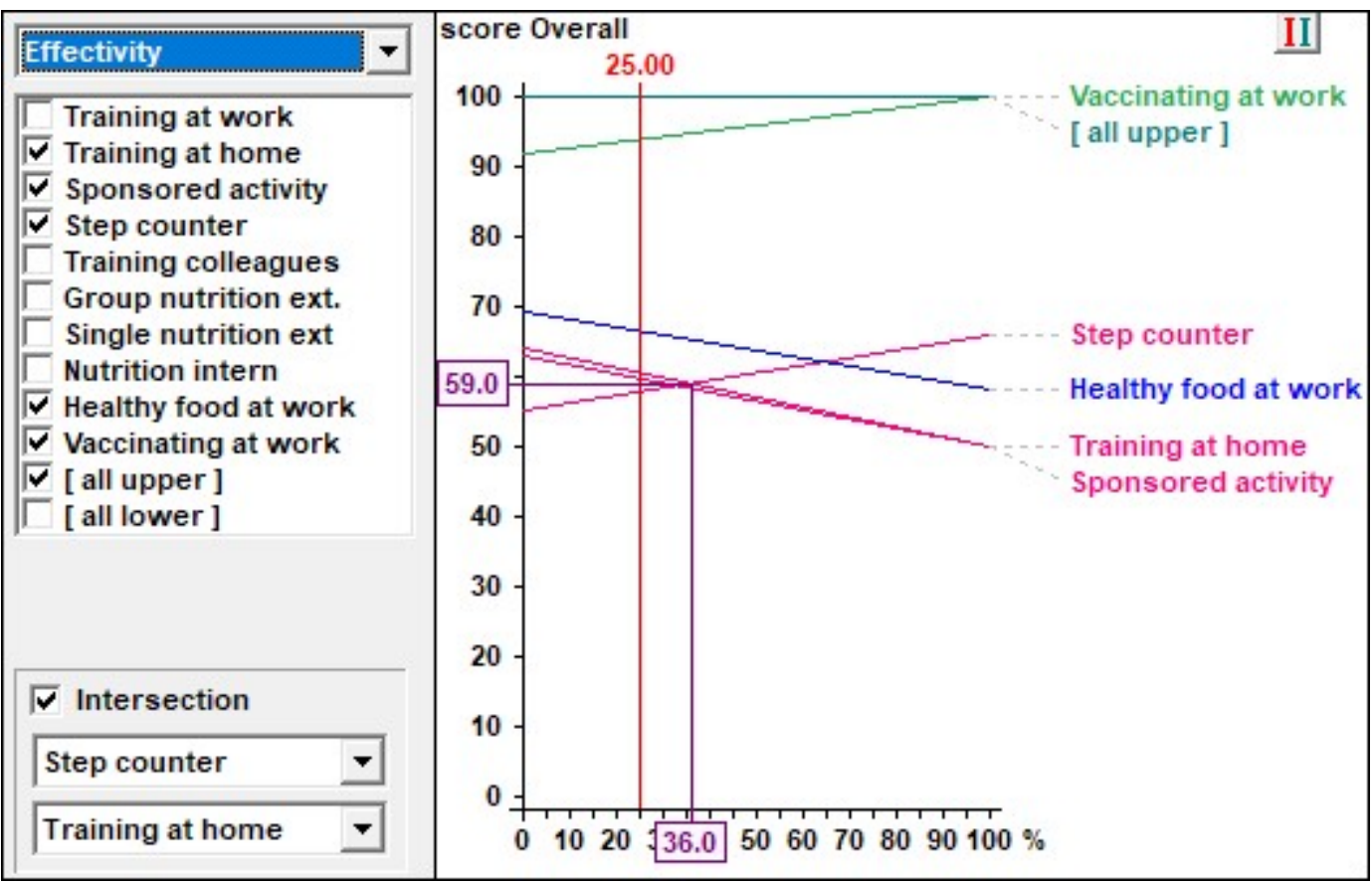

Figure 39: Sensitivity and robustness check for "Effectivity"

The following figure shows that the proposed training measures with regard to effectiveness are robust against any changes in weighting. The priority sequence does not change, regardless of whether the red weight line is shifted left or right. There are no intersections of the lines assigned to the activities.

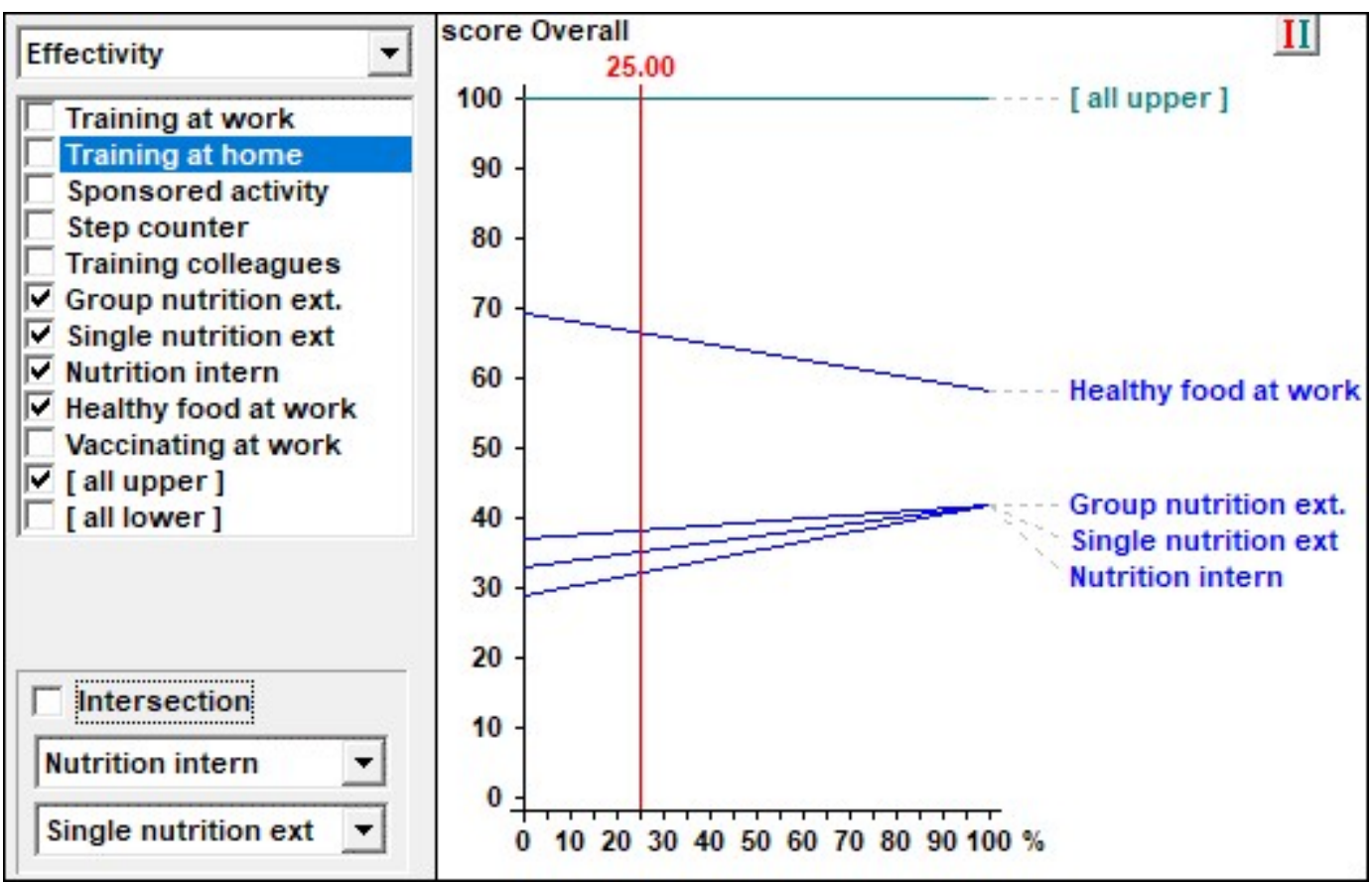

Figure 40: Sensitivity and robustness check for "Effectivity" and "Nutrition" 


\subsubsection{Sensitivity versus changes in the cost level weighting}

Effectiveness and cost level are both weighted at $25 \%$. Since vaccination is one of the most cost-effective measures, a change in prioritization against other measures is not to be expected. This is also confirmed by the sensitivity analysis. Looking at the graph for the cost-sensitivity of the training measures shows that only a change would be expected if the weighting (unrealistic) of costs would fall below $16 \%$.

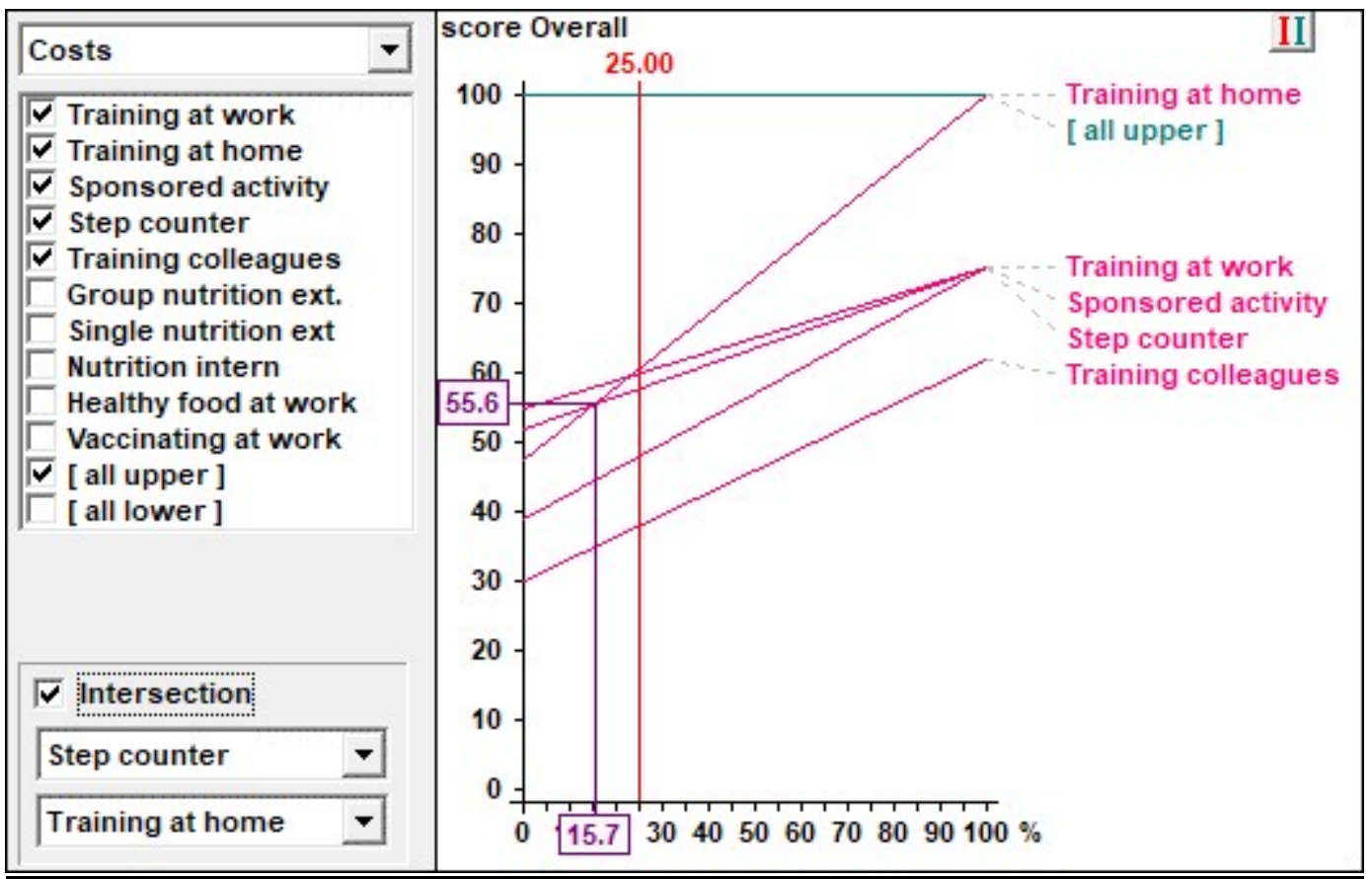

Figure 41: Sensitivity and robustness check for "Costs" and "Training"

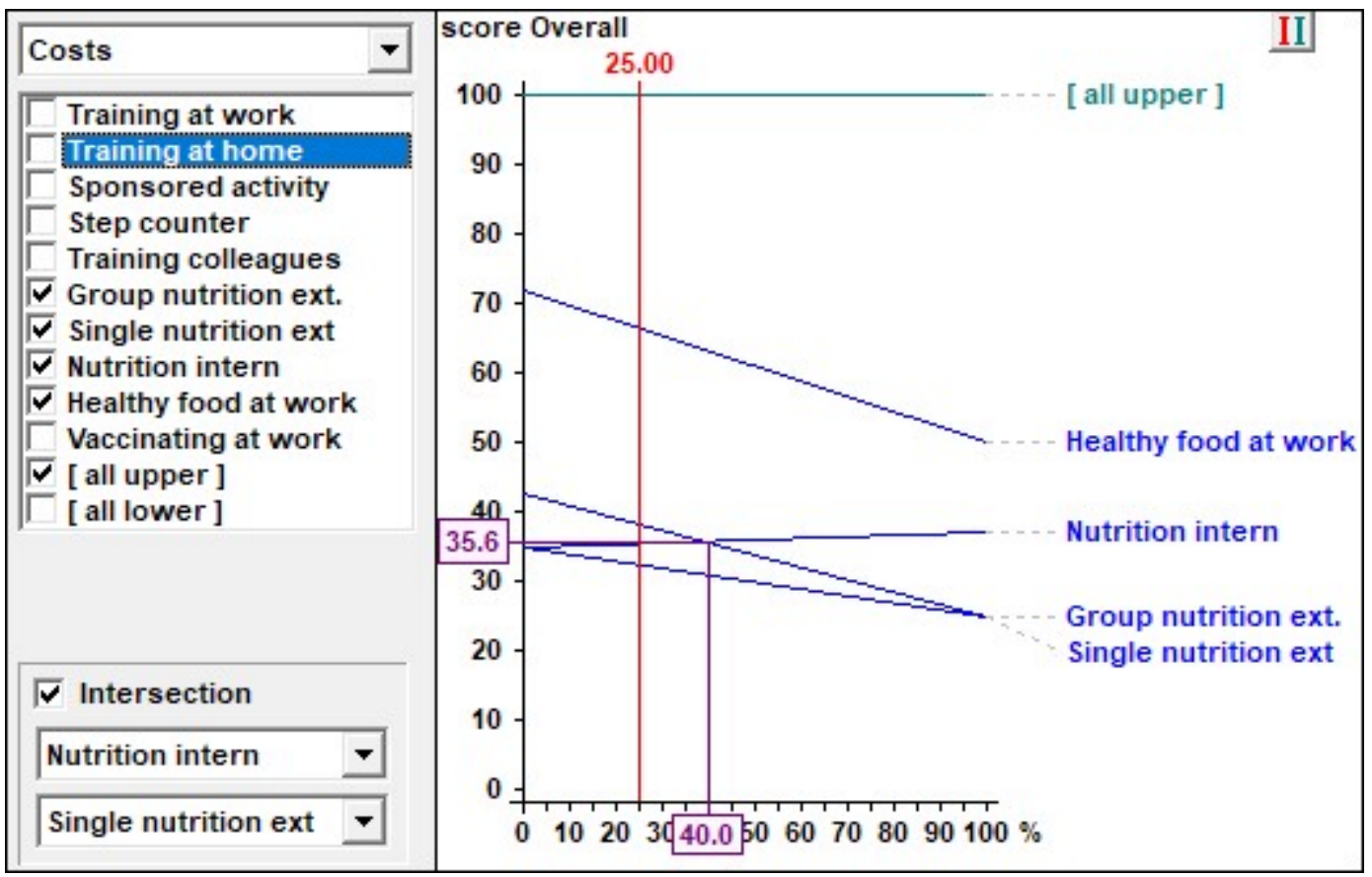

Figure 42: Sensitivity and robustness check for "Costs" and "Nutrition" 
If costs are gaining a higher impact on the decision, the ranking of the measure "Group nutrition consulting extern" decreases. If the weighting is $40 \%$, this measure falls short of the (unpopular) measure "Internal nutrition consulting", as the figure above shows.

\subsection{Hypotheses on scoring}

In chapter 4 "Methodology" the following hypotheses were made:

$H_{0}$ : MFAs favour the costlier of the individual programs for the promotion of health (i.e., the two features are correlated strong).

$H_{0}$ : Cost level and effectivity of the individual programs for health promotion are not independent (i.e., the two features are correlated strong).

$H_{0}$ : MFAs prefer the more effective of the individual programs for the promotion of health (i.e., the two features are correlated strong).

Another reason for the hypotheses tests was to determine whether the decision criteria are independent of each other.

In order to test the hypotheses, the following procedure was used: Firstly, for each combination of the decision criteria, a scatter diagram was drawn up. In addition, the so-called correlation coefficient, a measure of the possible relationship, was determined. A line, the so-called regression line, was entered into the scatter diagram. As explained in section 4.2.7, the comparison of observed values with expected values is required for the hypothesis test. For the respective null hypothesis, the expected value was assumed to be the value as obtained from the regression line.

The first null hypothesis asserts that the MFAs prefer cost-intensive measures. The more expensive a measure is, according to the hypothesis, the more it is preferred. In order to check the assertion, the following scatter diagram was created with the columns "Costs" and "Acceptance" from Figure 35.

In the scatter diagram, a blue dot is entered for each measure of the priority list. On the $\mathrm{x}$-axis the cost performance and on the y-axis the acceptance. "Training at home" has, for example, the coordinates $(100 ; 46)$ - it is cost-effective with medium acceptance. With the straight line, which is likewise colored blue, the trend line or regression line, an attempt is made to find a straight line with which the blue points have as small a distance as possible. The corresponding straight line formula is shown in the diagram at the lower right.

Using this formula, a table of the expected values can be compiled. According to the formula, the expected acceptance value of $y=0.3338 * 62+29.07$ results for a cost value of 62. The correlation coefficient is 0.48 (i.e. a weak corelation). Table 37 contains, for all existing cost values from the priority list, the values calculated with this formula and thus expected, assuming that the null hypothesis applies. 


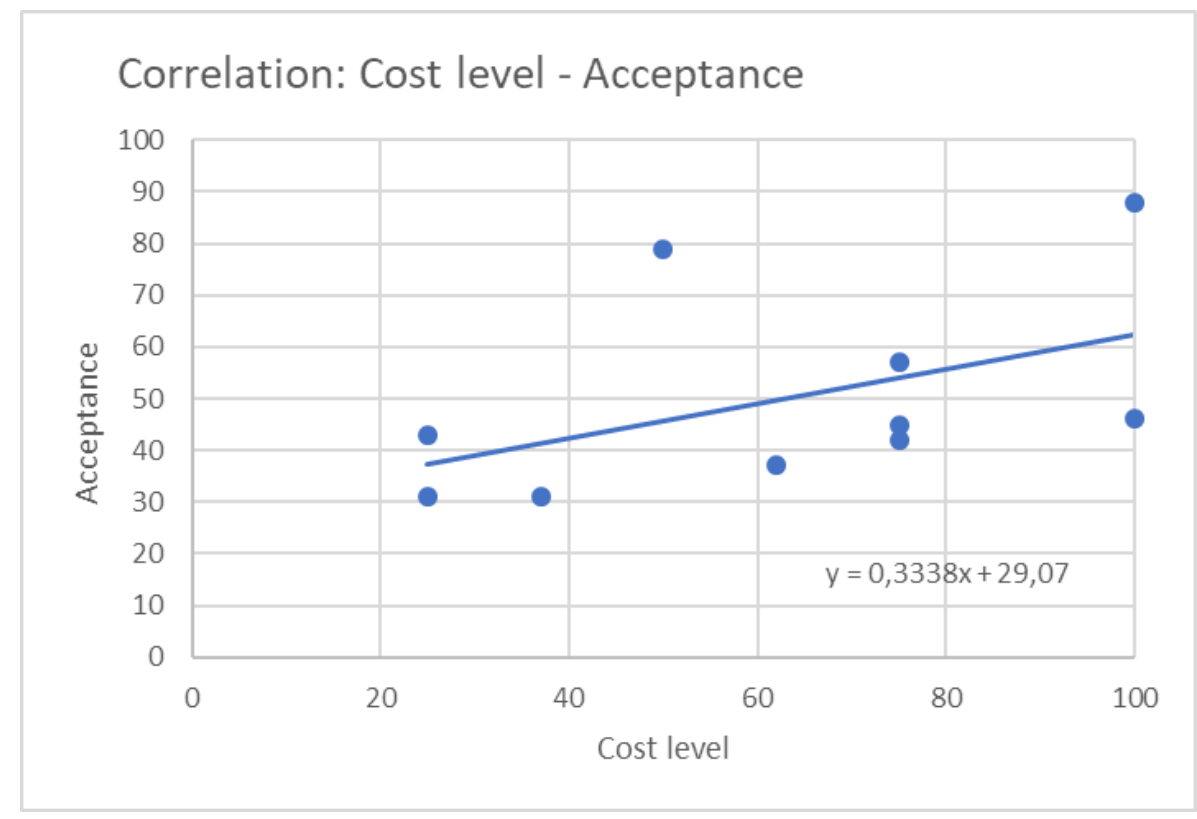

Figure 43: Scatter diagram for the correlation: Cost level-Acceptance

\begin{tabular}{|l|r|r|}
\hline & Cost level & $\begin{array}{c}\text { Expected } \\
\text { Acceptance }\end{array}$ \\
\hline Vaccinating at work & 100 & 62 \\
\hline Healthy food at work & 50 & 46 \\
\hline Training at home & 100 & 62 \\
\hline Sponsored activity & 75 & 54 \\
\hline Step counter & 75 & 54 \\
\hline Training at work & 75 & 54 \\
\hline Single nutrition ext. & 25 & 37 \\
\hline Training colleagues & 62 & 50 \\
\hline Nutrition intern & 37 & 41 \\
\hline Group nutrition ext. & 25 & 37 \\
\hline
\end{tabular}

Table 37: Data for testing the null hypothesis on cost-> acceptance

This table was used for the chi square test with the result that the null hypothesis can be rejected at a very high level of significance $(p<0.1 \%)$ and therefore the counter hypothesis $\mathrm{H}_{1}$ applies:

$H_{1}$ : MFAs do not favour the costlier of the individual programs for health promotion (i.e., the two features are not correlated strong).

The counterhypothesis also applies in the direction of acceptance at the cost level. That is, higher/lower acceptance is not associated with higher/lower costs.

The null hypothesis for the relationship of the cost level to effectiveness and vice versa was tested in the same way. If the null hypothesis were applied, it would mean that more expensive measures are more effective. This null hypothesis can also be rejected at a statistically highly significant level. The following counter hypothesis applies: 
$H_{1}$ : Cost level and effectivity of the individual programs for health promotion are independent (i.e., the two features are not correlated strong).

Another picture results when the null hypothesis is tested for dependence on effectiveness and acceptance. Even the scatter diagram and the high correlation coefficient of 0.77 suggest that the null hypothesis cannot be rejected. The high p-value ( $p>38 \%$ ) confirms this so that no statistically relevant statements can be made about the correlation between the effectiveness and the acceptance of the measures by the MFAs.

As a possible cause of a causal link, it can be assumed that the MFAs may have a high degree of knowledge about the effectiveness of the measures due to their training and experience. Especially when vaccinating, the effectiveness of the measures is intensively informed, so that the "reputation" of the measure determines its acceptance. On the other hand, the estimation of efficacy here is based on studies that are not well-known, which would lead to a causal link. Similarly, there is a presumption of causality when one assumes that the MFA prefers the measures which they presume to allow their physician to express an appreciation when given to them.

Also, the reverse causal link, namely, that the acceptance affects the effectiveness is not excluded. The more a measure is accepted, the higher is the chance that it may prove effective. The "belief in success" in the sense of a placebo effect and the "selffulfilling prophecy" addressed here would be the explanatory patterns.

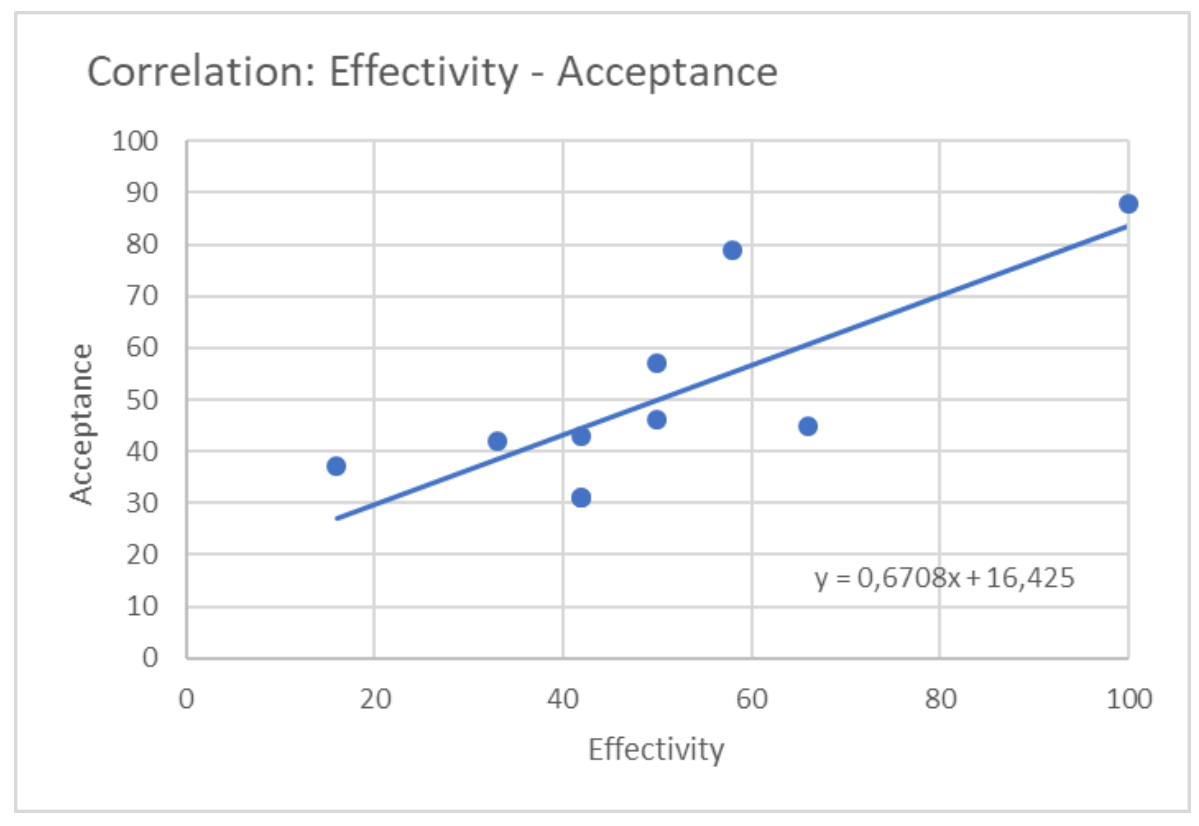

Figure 44: Scatter diagram for the correlation: Effectivity-Acceptance

\subsection{Summary of results}

The following points summarize the research findings of the development of a priority concept. 
They contain the result of the scoring, the sensitivity tests, and the essential hypotheses and show the contribution to knowledge for the area "Decision-making in German general practitioners' surgeries to activities promoting personal health potentials".

Moreover, the research question

3. On what basis can the implementation of these activities be prioritized by a multiple criteria decision-making model, which considers relevant stakeholder perspectives?

is answered:

- The three key stakeholders' perspectives staff (MFAs), medical professionals (general practitioner) and owners (managers) are covered by the decisionmaking criteria for acceptance, effectiveness and cost level.

- For each of the decision-making criteria, acceptance, effectiveness and cost level, it is possible to develop comprehensible and largely reliable assessments, which enable a prioritization.

- The decision-making criteria for acceptance and cost level as well as cost level and effectiveness are statistically significantly independent of each other. A link between acceptance and effectiveness cannot be statistically significantly confirmed or rebutted.

- The assumption that $M F A s^{\prime}$ preferences are based on favouring costlier measures must be rejected. The assumption that MFAs prefer to take measures with high effectivity can neither be rejected nor confirmed.

- The only measure that achieves more than $50 \%$ of the performance points for all three decision-making criteria is "Vaccination at work". It is the measure that should be taken with the highest priority. The recommendation is robust; even with changes in the weighting, it remains the first rank.

- Measures that are best for a one-dimensional view:

- Healthy food at work: Acceptance - "What matters most for the staff."

- Training with step counter/fitness watch: Effectivity - "Main thing it helps."

- Training at home: Cost level - "But it must not cost anything."

- Measures which, in two decision-making dimensions, account for more than $50 \%$ of the points are:

- Healthy snacks/food at work: Acceptance and effectiveness

- Sponsored activity: Acceptance and Cost level

- Training with step counter/fitness watch: Effectivity and Cost level 
- The sensitivity and robustness test also show high stability in the priority list for non-vaccine-related measures. Only fundamental changes in the weighting lead to significant changes in the ranking. 


\section{Discussion}

This chapter looks at how to realize the findings into a general practitioner' surgery, that is, the implementation. As Figure 18 shows, this is the last step in the multicriteria decision-making model. The first section presents the results of the qualitative interviews general practitioners about the established priority list and possible implementation concepts. The concept proposed as a standard is discussed regarding its adaptability to the individual general practitioner's surgery.

Thereby, three consecutive approaches are discussed.

The first sees the promotion of MFA personal health potential as a more or less unique, situational action. The setting is: The general practitioner is convinced of the correctness of the concept and now plans to implement it as quickly as possible in his own practice without any further implications. The implementation aspects to be considered are presented in section 1.

The second setting sees the standard proposal on primary prevention as a first key step towards a more comprehensive and ambitious doctor's health management project, based on the recognition that employee health can be a key success factor for the practice and, in the longer term, not to be underestimated represents a competitive factor. This section illustrates what steps still need to be taken to meet the requirements of a sophisticated health management system.

From a holistic view of practice management, health management can be embedded in a more comprehensive quality management system. This third setting outlines the relationship between this study and the establishment of a quality management system.

All three settings were addressed in the interviews with general practitioners and discussed as alternatives.

While the three settings emphasize the content aspect of an implementation, the last section deals with the psychological contract that represents the processual aspect. It is worked out what requirements its fulfilment places on the general practitioner.

\subsection{Results of structured interviews with general practitioners}

As described in 4.3.8, structured interviews were conducted in November and December 2017 with four different general practitioners. All interviews were conducted using the interview guide as presented in Appendix 5. The aim was to reflect the results of the MFA surveys and to discuss the introduction of health promotion measures for MFAs. The time required for each interview was limited to one hour each. Answers were documented in Appendix 5. 


\subsubsection{Characteristics of the interviewed general practitioners' surgeries}

It goes without saying that four qualified interviews cannot form a representative basis. Nonetheless, the interviews conducted were very enlightening and, regarding the insights gained in health management, characterized by a high degree of diversity. This was not least because the four selected interview partners had four fundamentally different characteristics. To simplify the reference to the different types, they have been labelled by the author with succinct names.

General practitioner's surgery A: in the following called "classic" - is a classic general practitioner's practice with an older, doctorate holder (64 years) in a prosperous suburb of a German city, characterized by good middle-class relationships. Employed is a full-time MFA (55 years old), supported by one to two helpers plus a helping husband. The range of services includes acupuncture and naturopathy as a special feature. There are no special offers for primary prevention. Also, the MFAs do not perform primary prevention or primary care tasks. Due to illness absence often leads to increased burden of work and longer working hours of the assisting husband. As health-promoting measures a stress-free work environment as well as free acupuncture and naturopathic advice by the owner are highlighted.

General practitioner's surgery B: hereafter referred to as the "young doctor" - is a general practitioner and internist with a doctorate. He is relatively young (age 35 ) and has recently taken over a practice in the centre of a German city. As an additional education he has a management qualification. Under his leadership, three other doctors are working, supported by a total of eight part-time MFAs. In addition to acupuncture, homeopathy and naturopathy, travel and tropical medicine services are offered. Prevention and preventive medicine are a focus of practice in which tasks are also delegated to the MFAs, with special mention being made of NLP (Neuro-Linguistic Programming) activities. In the MFA tasks of primary care were expressly called blood glucose and blood pressure measurement. Absenteeism is rare and may be recorded and evaluated.

General practitioner's surgery C: hereafter referred to as "Holistic" - is a family practice for general medicine and naturopathy with a female owner with a doctorate (51 years) on the outskirts of a German city. Patients come from all walks of life and include children and adolescents cared for by a second female doctor. One full-time, five part-time MFAs and two trainees (18 to 45 years) support the doctors, who mention acupuncture and numerous naturopathic treatments, as well as yoga as a special performance focus. The involvement of the MFAs is an integral part of the practice concept, which is very strongly geared to the individuality of the patients but also explicitly to the individuality of the MFAs. More detailed information can be found in section 7.5. Tasks of primary care are "barely" performed by MFAs. Abnormalities due to absenteeism often occur; absenteeism is recorded via the time recording system, but no 
cause-related evaluation takes place. The range of practiced health-promoting measures ranges from "rest periods", "drinks", weekly "fruit and vegetable box", sporadic yoga to "fresh air during lunch break" (in the nearby park).

General practitioner's surgery D: hereafter referred to as the "Experienced" The owner (64 years old with doctorate) has set up and expanded a general practice in the city centre of a large German city with several employed doctors. The principal areas of activity include occupational medicine and, as so often associated with it, travel medicine. Three full-time and five part-time MFAs of all ages are employed. Primary prevention offers are part of the standard range of services complemented by a comprehensive range of medical check-ups. The delegation of primary prevention tasks to MFAs is planned (to a limited and still to be determined extent). General medical care tasks are not part of the range of services. Disorders due to illness-related absences occur, are also recorded in time, but not evaluated due to labour law concerns. The need for health promotion measures for the MFAs is recognized, but - except for vaccines - insufficiently implemented.

It is striking that three of the four interviewees specifically call acupuncture as a service offering, which is probably because in addition to the belief that acupuncture can heal and relieve pain, the costs are covered by the statutory health insurance companies. The "Experienced" (D) expressed that he did not want to include these points in the range of services given his age. The same applies to naturopathy, however, there is due to the wide range of medical treatment no continuous coverage of all health insurance guaranteed.

The concept of "delegation of tasks of primary prevention to MFAs", as set out and explained in subsection 3.2.2, seems to have reached the doctors. Except for the "Classic" this was confirmed as a useful concept. A consultation with the "Classic" confirmed that the assistant was a "doctor's receptionist" who was not trained as a MFA with an expanded job profile.

\subsubsection{Comparison with the priority list}

The second part of the interviews was used to compare the ten proposed measures in the priority list with the ideas and experiences of general practitioners. First, it was asked if measures and/or decision criteria were missing.

As was the case with the open questions of the MFA Survey, first check-ups were mentioned as a missing measure, but here again, as it turned out, they could not be classified as primary prevention measures. Also, in the proposal "team events (excursions, celebrations, etc.)" showed the discussion that it is more likely to promote the working environment and thus increase the psychosocial well-being, as the primary prevention. (If it were, otherwise, the health insurance funds, which pay direct primary prevention measures, would also have to contribute to the costs of company out- 
ings and Christmas parties.) The suggestion "sugar-free drinks in the workplace" is not explicitly mentioned in the priority list but can be subsumed to "Healthy snacks at work". The same is true for the suggestion "Promoting self-initiative", which can be flexibly classified under "Sponsored activities after work".

In the decision criteria, none was considered missing despite an explicit inquiry. The weights for acceptance / effectiveness / cost level were distributed as follows:

$\begin{array}{ll}\text { The Classic } & -30 / 30 / 40 \\ \text { The Young doctor } & -40 / 40 / 20 \\ \text { The Holistic } & -33 / 33 / 33 \\ \text { The Experienced } & -70 / 25 / 5 \\ \text { Average } & -43 / 30 / 27 \\ \text { Priority list } & -50 / 25 / 25\end{array}$

The overview shows that the weighting used for the development of the priority list and the average for the structured interviews are very close to each other.

Acceptance is rated relatively low by "The Classic". This reflects the attitude "Health is not something you can manage" (translated by the author). "The Experienced" with the highest weight emphasizes that "... you always have to talk to the MFA first ..." (translated by the author), then adds: "But even with low acceptance I offer measures, if I am convinced." (translated by the author). "The Holistic" finally sees the MFA Survey as a regular task.

The effectiveness is considered by the "The Young doctor" as important as the acceptance. While the relative low weight of effectiveness of the "The Experienced" and "The Classic" reflects years of experience with efficacy claims.

The cost level only plays a dominant role for "The classic". This is due to the size of the practice. "We have to look where we are staying, and I do not like investing in measures that I do not know if they will do anything." (translated by the author) This quote proves the high weighting of the cost level in this case.

The individually adopted weighting scheme was combined with the table in the interview guide. For each of the ten measures the assumed acceptance, the effectiveness and the cost level were estimated in the interviews. Compared to the priority list, the following results are obtained:

"Vaccination programs" are also ranked number one in the GP's evaluation of the priority list, but the distance to the other measures is not as clear as in the evaluation of the MFA survey. This is probably because one of the practices is more likely to be seen as vaccine-critical, at least for flu vaccines, and that the assessment of the costs was not as extremely favourable. Inquiries showed that answering the question the general practitioners has forgotten that all costs are covered by the health insurance also in the vaccination of own employees. 
For the measure "Internal Nutrition Advice" the GP interviews rank 2nd, while the same measure is only ranked 9th in the priority list of MFAs. This is due to the large differences in all three decision criteria. For example, the difference in acceptance score is 38. While the MFA survey's acceptance is only 31 points, the surveyed GPs count 69 points. The costs are also estimated to be higher than comparable market values. The effectiveness - in the priority list based on the literature research - is estimated to be much higher (56 points instead of 42 points). The high rating reflects the high estimation of one's own performance. In addition, one of the four doctors interviewed has additional training in nutritional medicine, so he may be inclined to rate the impact of healthy eating particularly high.

If one ignores "Internal Nutrition Advice", as this may pose a problem, as explained in the previous paragraph, "Healthy food at work" is ranked second in both analyses.

The readiness for the measures "Vaccination programs" and "Training at home" differs most in GP interviews and MFA survey. Vaccination is $88 \%$ percent preferred in the MFA survey, while the GP evaluation shows $56 \%$. According to the MFA survey, $46 \%$ would approve of "Training at home", while the general practitioners assume $31 \%$ as acceptance rate. Conversely, the measures "Internal Nutrition Advice" and "External Nutrition Advice in Groups" are overestimated. This means, the general practitioners surveyed believe that the measure is popular, while in fact it does not receive any special appreciation from the MFAs.

The evaluation of the differences between "assumed acceptance" in the GP survey compared to the preference profiles of the MFA survey is revealing. This may be due to the specific circumstances and peculiarities of the individual general practitioner's surgery, but at the same time it is a clear indication of the need for an employee survey when measures are introduced.

\subsubsection{Factors influencing productivity and performance}

In this part of the GP interviews, physicians were asked to rate the importance of key influencers and levers as shown in Figure 10 for the reduction of absent days. The levers proposed were primary prevention, work organization, workflow and leadership quality and competence, that is, the elements that can be directly influenced by the general practitioner and that then affect productivity and performance.

The table below summarizes the discussion results. The respective answers were discussed and then classified by agreement into the categories "low (4) / medium (3) / high (2) / very high (1)". 


\begin{tabular}{|l|c|c|c|c|c|}
\hline & Average & $\begin{array}{c}\text { The Young } \\
\text { doctor }\end{array}$ & $\begin{array}{c}\text { The Experi- } \\
\text { enced }\end{array}$ & The Holistic & The Classic \\
\hline Job design & 1.8 & 1 & 3 & 2 & 1 \\
\hline Work design & 2.0 & 2 & 3 & 1 & 2 \\
\hline Leadership & 2.8 & 3 & 1 & 3 & 4 \\
\hline Prevention & 3.5 & 4 & 3 & 4 & 3 \\
\hline
\end{tabular}

Table 38: Importance of levers for reducing absent days - extracted from the interviews with the general practitioners

The importance of primary prevention measures for MFAs has been consistently rated as low, even though they were considered as a first good-to-be-heard initiative. With "The Classic" and "The Holistic", the viewpoint of seeing primary prevention as a contribution to productivity and performance collided with the professional selfimage. The situation is similar with the self-image as a leader and manager: "That does not matter as much as the individuality of the employees" (translated by the author). The importance of a good working environment (referred to in the impact network as psychosocial well-being) is consistently confirmed, although leadership quality is not seen as a decisive factor, as is the health-promoting organization of work organization and job design.

As a further starting point for performance improvement, in addition to measures for team building, it was suggested that new or improved opportunities be created to find short-term MFA substitutions.

\subsubsection{Implementation of health management}

The first question was why a medical practice should introduce a health management system compared to other organizations. The "Young Doctor's" quote "A company that works with people's health should embody this through healthy employees" (Appendix 5 B 4.2 - translated by the author) expresses the core idea. The note "Employees learn from it and patients become role models" (translated by the author) also shows the potential marketing aspect in addition to the organizational aspect. "The Holistic" general practitioner points to the "limitations of a small business": ".. but I do not want to give additional staff additional tasks and burdens in addition to our 'everyday madness'”' (Appendix 5 C 1.5 mail - translated by the author).

The possible implementation concepts are discussed in more detail in the following sections of this chapter. Two quotes are made to point out possible introduction difficulties: "Ever since my additional qualification in psychosomatics, it has become clear to me that health management has too many manifold aspects to develop a catchy and coherent concept." (Appendix 5 C 4.3 - translated by the author) - "It depends on the personal relationship. Health is not a management object. It is enough if the businesspeople have already decided in the hospital. Doctors are not managers. " (Appendix 5 A 4.3 - translated by the author). 
Similar ambivalences occur in the question of the concepts propagated by some medical associations, such as "healthy leadership", "health culture in physician's office" or "health literacy MFA". The span ranged from "... I consider exaggerated, colliding with my understanding of the physician ... I see with discomfort the development at the medical council" (Appendix 5 A 4.4 -translated by the author) up to "It would be good if these concepts were better known and implemented ..." (Appendix 5 D 4.4 - translated by the author) or "Could have a (medium) contribution to the practice afford." (Appendix 5 D 4.4. - translated by the author).

\subsection{Implementation of the standard proposal}

In this section, the implementation is developed as a "stand-alone measure". It is assumed that the general practitioner considers the standard proposal to be adequate and that implementation aspects are the focus of attention.

In three of the four interviews this was felt to be the most obvious suggestion. "The Holistic" is reluctant to use standards and sees the promotion of personal health potential as a process: "In our practice, this is an ongoing process that involves many individual discussions. Employees are individuals." (Appendix 5 C 2.5 - translated by the author).

The time required to work out a concept like the standard proposal was estimated at eight to ten hours. If the standard recommendation is used, the effort is reduced to two hours according to the estimates of the general practitioners.

The result from a multi-criteria-decision analysis is not one solution. It provides a list of possible solution sorted after priorities. This is important for the use in the practical work of general practitioners' surgeries. These organisations have limited resources of time and money to improve their health management. To avoid inefficient health management program or programs which are not accepted by their employees, the research findings for the development of a priority concept in chapter 6 are important. The priority list (Figure 35,36) also respects the different financial possibilities of general practitioners' surgeries. They can individually decide if they implement one or more options. If one option is too expensive the next activity could be selected. This structure allows a result which is very close to the practical work. None of the studies provides a suggestion for the one best activity and no organisation is forced to use this activity. The result depends on the individual weighting of each element and it is still useful in the daily work if it is adjusted to the individual needs of one organisation.

However, the promotion of personal health potentials is an ongoing process and the list as standalone To-Do-List could be not enough.

The recommendation for the practical use is the following 
1. Commit yourself as an employer to the goal of health management: Improve the health of employees

2. Think about the budget for the implementation

3. Define status

4. Coordinate the activities with the employees

5. Implement activities

6. Be patient

7. Measure the result

At first the employer needs to make a personal decision. It seems that the promotion of personal health potentials leads to a higher performance and more profit, but it is a long-term process and this research did not find evidence that the result guarantees a positive return of investment. This means any general practitioners' surgery needs to think about the investment they want or can make in the promotion of personal health management and if they can handle the uncertainty of return. It will cost resources. Afterwards the status about the actual primary prevention is needed. The commitment with the employees is important to support the implementation of the activities. This could be done with the provided questionnaire or through personal discussions with the employees. After this the activities from the list could be implemented. In the best case this are all activities, but this will often be too difficult or time consuming. However, every activity is effective and even one implemented activity goes in the right direction. The implemented activities need time to work until they produce an effect. The best rated activity of vaccines will need at least one flu season to provide a result. Monitor the results in the organisation. These results will be measurable through the number of absent days, but it is important to document them and to exclude unexpected events e. g. through accidents in the private life.

If the general practitioner wants to become active in all three areas of vaccination, movement and nutrition, the following paragraphs provide concrete implementation instructions for the three most prioritized measures.

\subsubsection{Specificity of vaccination programs}

In Germany, there is no vaccination requirement, so that participation in vaccination programs is voluntary. At the same time, vaccination is clearly the most effective measure with the highest detectable effect on days lost.

There are several studies, which proved that for example a flu vaccine reduced the average of absent days. The study "Occupational vaccination of health care workers: Uptake, attitudes and potential solutions" (Little, Goodrigde et al., 2015) and the study "Healthcare workers and immunity to infectious diseases" (Vagholkar, Ng et al., 2008) provide evidence to have a very strong and positive influence on the absent days.

Although the coverage rate for MFAs is above average compared to other occupational groups, there are still two gaps (5.2.5). First, the difference between "private 
vaccination" (75\%) and "vaccine at work" (88\%). Here, through education and information by the doctor, a considerable potential can be opened relatively easily by clearly communicating the offer for vaccination in one's own practice. On the other hand, analysing the open questions reveals that around 5\% of MFAs have unclear ideas about the effects of vaccinations such as "My immune system is strong enough". Again, an open discussion with the general practitioner could help.

Specifically, with regard to flu vaccination, some MFAs are uncertain or ignorant that they belong to the risk groups in several respects in the sense of the Standing Vaccination Commission (STIKO, 2017), the German immunization agency at the Robert Koch Institute RKI (2017): "Vaccination should be given as part of an increased professional risk to

- persons at increased risk (for example, medical personnel) and

- persons in facilities with extensive public traffic

- persons who can act as a potential source of infection for those at risk.

This vaccination recommendation shows the MFA's own risk of becoming ill, the risk to other persons acting as germ carriers and the impairment of patient safety (e.g. older people with weak immune systems).

Since, as mentioned earlier, flu vaccines are new every year and, unlike many other vaccines, no permanent protection is built, the challenge is to motivate each year again.

\subsubsection{Design of the measure "Healthy Snacks"}

The cross-group popularity of the measure among employees was noted in paragraph 5.2.4.1. With regard to the effectiveness and the cost level, this measure cannot score very well.

The effectiveness, as understood in this research, is mainly focused on long-term effects. The following article draws attention to the short-term, so to speak, everyday effect: "The possibility of providing light meals in the breaks, vitamins and minerals (e.g. through a fruit basket) ensures a much flatter drop in the performance curve over the whole working period, a less strong "midday low" and for intact defences of the employees. The investment in a fruit basket is also perceived by employees as a sign of appreciation. " (Barré, 2014).

But not every food and drink are suitable as a small snack. Although chocolate bars and other sweets deliver energy immediately, they quickly turn hungry again. A better alternative is nuts (are rich in protein, vitamins and minerals), fruits, sliced vegetables or a yogurt. The compilation of healthy eating offers at the workplace could be an opportunity for the employer to find feasible and attractive solutions in teamwork in discussion with the MFAs.

In a representative study, one of the largest health insurers in Germany, Techniker Krankenkasse (TK), compared the eating behaviour of employed persons between 
2013 and 2017 (TK, 2017). When asked what their nutrition is all about, 45 percent of respondents said they wanted to eat healthy food first and foremost. In the last survey on the dietary behaviour of people in Germany in 2013, only 35 percent said so. For the first time "healthy" is more in demand than "delicious" (41 percent). The criteria "low-calorie" and "fast" are also losing relevance. This trend is visualized in following figure.
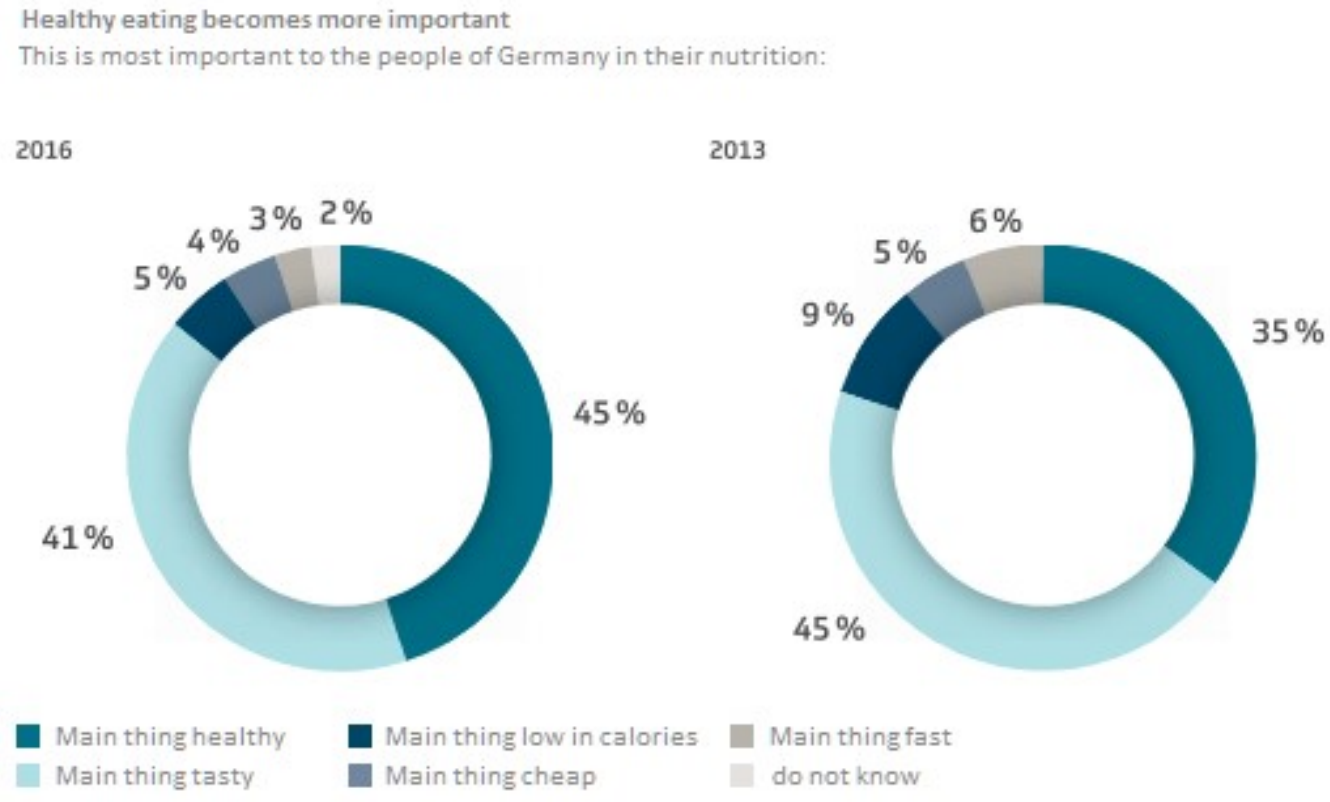

Figure 45: The trend to healthy snacks at work (TK, 2017 -translated by the author)

Despite the trend change, the change in nutrition remains a difficult matter. In the same study, participants were asked about the reasons that stand in the way of a healthy nutrition. "To feed myself healthier, I lack ... time and rest (56\%), staying power (46\%), will (43\%), money (29\%), cooking skills (28\%), better working conditions $(26 \%)$, knowledge (25\%), family support (22\%), advice $(21 \%)$, interest $(2 \%)$, nothing - already eat healthy (10\%) " (TK, 2017 - translated by the author). For the general practitioner's surgery that is willing to implement, this makes it possible to identify possible obstacles in the implementation.

At another point in the study, one-quarter of women say, "they do not have time to take a break and eat on the side". A circumstance that could also apply to the most hectic practice operation (and perhaps even higher.)

\subsubsection{Design of the measure "Training at Home"}

"Training at home" (cf. preference profile 5.2.3.4) is among the measures with the least variation within the age group. To explain this, one should first consider the characteristics of the measures "Training at workplace", "Step counter" and "Training with colleagues". In contrast to "Training at home" they limit the customizability. Training in the workplace is due to the spatial limitations; not for all ideas there is a 
suitable environment, e.g. for laying out a yoga mat. Step counter means restricting the variety of movement options to walking and/or jogging, and "training with colleagues" requires responding to the ideas of others. "Training at home" as well as "sponsored activities" clarify the need for individuality.

Therefore, any general practitioner willing to implement should approve any idea of a personal exercise program that results in exercise and movement. The support can take many forms and differ significantly from employee to employee. In detail, one could mention:

- Paying for DVDs and other media with a focus on exercise and gymnastics programs (e.g. Zumba, aerobics, ski gymnastics, stretching, back exercises, ...)

- Paying for DVDs and other media with a focus on relaxation and concentration exercises as well as anti-stress content (e.g. Yoga, Chi Gong, Tai Chi, Feldenkreis, progressive muscle relaxation according to Jacobsen, ...)

O Paying for small equipment for physical activity promotion (e.g. dumbbells, stretch bands, sitting balls, home trainer, ...)

- Encourage to travel to work by bike or on foot, or at least to do sections of the way (e.g. the way to the nearest subway station) in this way.

○ Encouragement to recognize "opportunities for movement" (e.g. use stairs instead of elevators wherever possible)

- Encouragement to plan leisure activities also under the aspect of exercise training.

In principle, it can be recommended to show generosity in these areas, since these are not cost-intensive measures and the appraisal effect is in the foreground. In addition, medical advice and medical expertise is needed to design a home-based training program that can be tailored to the individual needs of the MFA.

As with all primary prevention measures, the role model of the physician is also important as it enhances credibility.

\subsubsection{Measures combinations}

Generally, the result of the literature review was that there is not one special activity, which leads to better primary prevention. The combination of several activities seems to be the best way for an improved promotion of personal health potentials. Van Strien \& Koenders (2010) provides evidence for this. Following this argumentation, a bundle of health activities needs to be tested. However, there is no result for such bundles in the literature review. It could be that the combination of options from all areas which are used can lead to better results than the use of one single action. 
The use of two or more activities should lead to a better result than one. However, there are no studies about negative interaction when several activities are used simultaneously. There is no hint for such negative interaction, but it would be possible for example, that a strict diet combined with sports can have such negative effects. Following this the recommendation is to implement more than one health activity.

The results from subsection 5.2.7 "Preference profiles to combined programs" can provide valuable information on the acceptance of the measures. Figure 36 "Priority list in a 3D overview" in section 6.4 shows starting points for the evaluation of combined measures according to different decision criteria.

\subsection{Adaptations of the standard proposal}

\subsubsection{Adaptation to age structure}

If a physician's surgery has a specific age structure, many young, middle or older MFAs, it does not need to make any special arrangements for the first three measures proposed in the priority list. The acceptance of these measures is independent of age.

Only in the case of the following three measures should the age structure be considered as a plan-relevant factor (5.2.3, Figure 23):

- "Training within a group with colleagues" is significantly less preferred by the age group 26-35 years than in the other age groups - It is possible that this age group may feel under-challenged, because within the peer group there are hardly any differentiations.

- "Step counter and Fitness-Watch" are clearly not desired by the age group 56 to 65 years. - If the implementing general practitioner strives for these measures, for example because she/he is convinced that this provides a good means of steering and controlling to promote the effectiveness of the measure, he will have to reckon with the resistance of employees of the age group 56-65 years.

- "Training at workplace" is significantly more preferred by the age group 56 to 65 years than in the other age groups. - This measure, in contrast to the above, suggests that the older employees have a closer connection to the social environment of practice. A physician who has an interest in pursuing the consistent implementation of a movement program, because, for example, he is convinced that the consistent and lasting implementation is crucial. The implementation in the workplace ensures the permanent participation of the employees.

\subsubsection{Adjustment to cost situation}

To adapt to specific cost situations, it is advisable to look at the sensitivity analysis for changes in the cost weighting (Subsection 6.5.3). 
Figure 41 on sensitivity and robustness shows for the group "physical activity promotion" that even with a significant increase in the cost level, the proposed "training at home" measure cannot be superseded by any other measure in the overall assessment, and the other measures of the group does not change their positions in the priority list.

A slightly different picture is shown in the group "Nutrition" (Figure 42). Although the suggested measure "Healthy snacks at work" cannot be supplanted from the top rank by weighting changes, but from $40 \%$ weighting for the cost level there is a shift in the further nutritional measures. The "internal nutrition consulting by the physician himself" then receives a better overall rating than the other two nutritional measures.

\subsubsection{Adaptation to acceptance}

Employee surveys are among the three most important tools for analyzing the state of health in German small and medium-sized enterprises, in addition to the analysis of reports on absenteeism (Lüerßen, Stickling et al., 2015). They form the basis for the targeted participation of employees in the design of health-promoting measures. Therefore, in forming the priority list, the acceptance was weighted at $50 \%$. The individual physician must decide for his practice whether he will follow this suggestion and thereby give the employees' preferences the highest weight in his decision.

As explained in subsection 6.5.1, the list of priorities is not sensitive to a lower weighting of the acceptance. Even if all three criteria are equally weighted (one third acceptance), the priority remains for the three measures proposed here. If the physician gives a significantly higher weight to the opinion of the employees, the "sponsored activity" is one of the first three measures to be prioritized and "Training at home" falls behind.

\subsubsection{Adaptation to effectivity}

The situation is similar with adaptations to different weights for the effectiveness. As far as nutrition measures are concerned, the physician does not need to think twice, as Figure 40 shows that no weight change leads to a change in the order of priority. In the case of training measures, changes only occur if the weighting rises above $35 \%$. Then the "Step counter and fitness-watch" measure will be added to the three most important measures. However, this can sometimes become a problem because, as shown in subsection 5.2.3, this measure tends to be rejected by the age group 5665 years (Figure 23).

\subsubsection{Adaptation to own ideas}

The adaptation to own ideas can be sought in several ways. To be able to make these adjustments, the data collection sheets for scoring results from Appendix 7 supple- 
mented with the priority list could be provided as Excel worksheets with completed entries. Below is shown how to proceed with individual changes if necessary.

- The implementing general practitioner or the MFAs propose their own measure, which is not included in the previous proposals. In the "Acceptance" worksheet, the proposed measure is added by overwriting in the "Further proposed measures" group.

- The acceptance of the individual measures is assessed differently than in the MFA Survey. In the column Score the other value is entered.

- The effectiveness is assessed differently. For example, recent studies may be added that demonstrate another efficacy. Accordingly, the number of studies contained in the Excel sheet and their "level of influence" should be changed. Likewise, the underweights for literature review (75\%) and the result for the GP interviews can be changed in the Excel sheet.

- The cost level is assessed differently. Here, too, the individual scores can be changed in the worksheet and the weighting between the two columns "experience and market observation" and "GP interviews" changed.

- The weighting between the individual decision criteria can be changed in the worksheet "Priority list".

The changes can be made either by the MFA or by the general practitioner. Only low Excel knowledge is required.

The question asked in the GP interviews about the willingness to adapt to own decisions to use software (Appendix 5 - question 2.6) was only supported by "The Young doctor". Everyone else saw it as "too much administrative work to the detriment of the patients", “... plus too much computer insecurity." (translated by the author).

\subsection{Development of a project "Health Management System"}

This section assumes that the general practitioner considers the standard proposal presented in the previous section as a first step towards the introduction of a health management system. In comparison with the cause-effect network on the target system of a health management system in the general practitioner's surgery (Appendix 5) and the Rhodes and Steers (1978) model of employee attendance (cf. 3.1.3), it is shown which next steps would make sense after the primary prevention measures have been started.

This was discussed in the interviews as a potential advancement and seen by "The Young doctor" and "The Experienced" as a promising approach.

Generally, it needs some effort for every organization, and so for a general practitioner's surgery to implement a health management system. The priority list is a help and it provides proven options for the health management with a focus on promoting 
personal health potentials. It does not provide a full-service implementation of a health management system. Such an implementation will need time and resources. The general practitioners' surgeries need to invest and spend money to improve the health management and finally to enhance profit by it.

\subsubsection{Target system and leadership ability}

In section 3.3.3 "Deriving priorities for German general practitioners' surgeries", the leadership ability was identified as one of the most effective factors for the successful introduction of a health management system (Table 2: Cross-impact matrix of the interaction of health management goals). Therefore "Development and permanent anchoring of the management system" according to Walter (2007) is in the foreground. Here, it would first be necessary to clarify the goals to be achieved with health management. In addition to the objective of "emotional well-being" and the associated "reduction of absence days" in this research, according to Lüerßen et al. (2015 - translated by the author) other goals like

- Increase job satisfaction

- Improving the mental health of the employees

- Strengthening the personal health competence of employees

- Increase efficiency / competitiveness / productivity

- Improvement of the working environment

- Improvement of the employer image

- Strengthening the personal responsibility of employees

- Quality improvement

- Reduction of the labour turnover rate

- Reduction of occupational accidents

may be desirable. Here, the general practitioners would be required, depending on management style in consultation with their employees, to develop goals and their relevance to their own practices, to agree and to prepare the implementation. The afore mentioned objectives were identified in a survey of SME companies $(\mathrm{N}=401)$ and would need to be reviewed to see if they are relevant to microenterprises such as a general practitioner's surgery. If necessary, cross-impact matrix would be expanded to include new targets and to complement the interrelationships.

In the same study (Lüerßen et al., 2015, p. 18 - translated by the author) finds the thesis "Leaders play a crucial role in the implementation of a corporate health management" with about $94 \%$ an overly clear advocacy, which can probably be adopted without too much restrictions for the general practitioner's surgery.

The challenge for the general practitioner relates to both indirect and direct leadership. "Indirect leadership of an organization's management refers to the organizational framework. Direct leadership, on the other hand, is personal-interactive. It serves to implement structural leadership strategies and controls employee behaviours. It 
depends essentially on direct leadership, whether employees are valued and acknowledged, if they feel that they are treated fairly and encouraged. (Imarinen and Tempel, 2002 - translated by the author)

In addition, leadership is always associated with exemplary function and thus exerts a considerable influence on health management. "In addition to their role in managing their own health behaviours, leaders are also a key factor in implementing workplace health management measures. They act as promoters of the approaches by not only being able to provide information, but also propagating and often implementing measures. Through their power in the organization they can legitimize change processes and provide necessary resources. ..."(Wunderer 2007, p. 160, cited in Braun (2016) - translated by the author). It is worryingly true that studies on the health behaviour of physicians suggest that they are difficult to set an example: "Studies on the result that physicians due to various specific work, scarce time and personnel resources, low levels of job satisfaction, low levels of social support as well as onerous treatment situations and decisions cannot be cited as examples ... " (Eikamp, 2015 - translated by the author).

In addition to the definition of targets and a health promotion-oriented leadership behaviour, the establishment of a controlling structure is necessary to be able to achieve constant improvements and to control the success of the measures. Without controlling the measure success, it is hard to speak of a management system. If the objective is to reduce absenteeism, at least a periodic review of the sickness rate should take place, a controlling instrument that is described in the (Lüerßen, Stickling et al., 2015) and is used by around 55\% of SMEs that already measure the success of health management measures. If further objectives are defined, appropriate indicators such as ROI or employee satisfaction index must be prepared.

\subsubsection{Further measures of primary prevention}

The results of this research refer to three areas of primary prevention: vaccination, movement, nutrition. According to the Prevention Report (Schempp and Strippel, 2016), "addictive substance use" and "stress management" moreover must be mentioned. Following the self-assessment of general practitioners presented in section 2.2.7, they are also among the areas in which general practitioners have sufficient competence, so that MFAs, after appropriate preparation, could also perform counselling tasks and, as described in 3.3.3 would contribute to an improvement in the range of services of the surgery. However, delegation readiness is not comparably high for these areas (Figure 9).

The addiction prevention was not included in the suggestions for health management activities, even though there are studies about positive effects of addiction prevention (Laaksonen et al., 2009). Indeed, the abuse of alcohol or drugs can have a negative impact on the health of the employees. Smoking prevention or alcohol abuse were not taken in the possible activities because no good prevention strategy or activity 
which could be used by the physician could be identified. Beside this it is not possible to provide anonymous help within a small organisation. There are only two or three MFAs and the general practitioner; if the MFA take part in an addiction prevention program this could hardly remain anonymous. For the MFA survey there had been an ethical dilemma too, because most participants would not have answered honestly, if they had been asked about addiction problems. However, if the individual physician can invalidate the above arguments for himself and his practice, he should include the control of addictive substance use in his health management program.

Finally, there is one study which provides a very good citation which points out a general dilemma of the health management in this point. Laaksonen et al. (2009) argue "Smoking and high relative weight were most strongly associated with sickness absence, while the associations of other studied health-related behaviours were weaker. The associations were stronger for medically confirmed sickness absence spells for which heavy smoking and obesity more than doubled the risk of sickness absence in men and nearly doubled it in women. ". The problem is identified but there are no activities or only activities with a very limited effect.

Similarly, stress management measures have the potential to increase emotional wellbeing. Following the explanations of the Literature integration in subsection 3.2.2, the emotional well-being of MFAs depends not only on the health-promoting measures as analysed in this research, but also on health-promoting work organization and job design, as well as the leadership ability of the general practitioners as the employer. Apart from being able to form stress-related as well as stress-reducing circumstances, the leader, his behaviour, and his relationship with him, are essentially responsible for the work organization and also the job design. As stated in Braun (2016) and Eikamp (2015), stress management measures in this area require at least a reflective attitude of the executive. If general practitioners wish to establish credible health management in their surgery, sooner or later they will need to incorporate these aspects into their health management system design.

The review of the acceptance of MFAs, as proposed in this research and the systematic inclusion of other decision-making criteria could prove helpful.

"With regard to measures to increase psychosocial well-being, employee coaching, anti-stress training, team training and discussions with other contact persons, each with just under $80 \%$ positive answers, are considered helpful. Such measures are also very popular in business practice and are considered to be particularly effective because they are interactive. "- (Braun, 2016 - translated by the author).

The effort to "stress management" is often considered high. However, it should not be overlooked that the "Mental Risk Assessment" and its documentation is one of the compulsory tasks of which even microenterprises are not excluded (ArbSchG). 


\subsubsection{Further measures to reduce risk factors}

In addition to primary prevention, there are other measures that promote the targets of holistic, comprehensive health management. Like the primary prevention, they can be subsumed under the term "reduction of risk factors". Specifically, these are accident prevention, occupational safety and hygiene measures as well as measures for ergonomic workplace design. Mental and behavioural problems as well as burnout symptoms are also risk factors.

If there is an activity, which provides a better protection of accident, there is no need to ask the employees, because this activity or change in the workflow has to be made. That is not a question of stakeholder involvement. There is no fix definition of health management and it could be argued that work safety is not health management. It could be an argument for this interpretation that in Germany the health prevention is made by physicians and the work safety by engineers with another qualification and a more technical engineering focus. Especially for general practitioners' surgeries, the brochure "Risk Assessment for Medical Practices" contains a comprehensive description of all risk factors (BGW, 2017).

Concerning infectious disease and other hygienic measures the general practitioners' surgeries are regularly monitored by several governmental institutions. They take care that anti-infection rules are followed. Every medical physician must take care that his surgery is clean and fits the regulations. The obliged quality management system in the general practitioners' surgeries supports this, too.

The area of healthy office equipment like ergonomic chairs or monitor filter was completely excluded from the MFA survey. The author found a strong and clear meta-study which provided evidence that in general there is no effect to health: "Workplace interventions to prevent musculoskeletal and visual symptoms and disorders among computer users: a systematic review" (Brewer et al., 2006). Following this study, the peer reviewed literature does provide few studies with good quality which provide evidence for the effects of office ergonomic interventions on musculoskeletal or visual health. However, it could be criticized to exclude the ergonomic, but this research did not provide studies for the use of ergonomic chairs or office equipment which could be transferred to the general practitioner's surgery and provide a proven positive effect.

The same argumentation is valid for health supporting clothes like orthopaedic shoes. For example, orthopaedic shoes may influence the health of an employee and in the end the absent days are decreasing but this assumption is an isolated single activity and no study is hardening the assumption.

The prevention of mental disorder was also not included; even it is one of the areas causal for highest absent rates. At German congresses of occupational medicines, the theme mental disorder burnout was discussed very intensive. Mental illness causes second most absence days and it is a growing problem for years (Grobe et al., 2011). 
The TK Health report states: "That [the lack of time to compensate for stress] does not remain without health consequences. For 15 years, absenteeism has been rising due to stress-related illnesses such as anxiety and stress disorders in all age groups, but especially among middle-aged workers. On average, the 30 - to 44-year-olds are absent 2.4 days a year due to mental disorders. No other diagnostic chapter is responsible for more absenteeism in this age group. " (Grobe, Steinmann et al., 2016 translated by the author).

\subsection{Integration of the proposed concept into quality management}

As shown in subsection 2.2.6, general practitioners are committed to quality management. Part of the medical profession is not only a chore but is also used by many doctors to increase the efficiency of the practice. This section briefly outlines the starting points for integrating the proposed concept into the quality management system. An approach, that was approved by all general practitioners in the interviews.

According to $\S 135$ a SGB V: "... to safeguard and further develop the quality of the services provided by them" ... "Contract doctors, medical care centres, licensed hospitals, providers of pension benefits or rehabilitation measures and facilities with which a health care contract exists ... are obliged ... to participate in inter-facility measures of quality assurance which aim in particular to improve the quality of results and to introduce and further develop quality management within the facility" (translated by the author).

On this legal basis, G-BA (2016) has developed a framework directive on requirements for in-house quality management for contract physicians, hospitals and other medical institutions. As goals are mentioned in it: "Quality management means the systematic and continuous implementation of activities designed to achieve sustained quality promotion in the context of patient care. Concretely, quality management means that organization, work and treatment processes are defined and regularly checked internally together with the results. If necessary, structures and processes are then adapted and improved. At the same time, the orientation of the processes to professional standards, legal and contractual bases in the respective institution should be supported. The benefits of quality management as an important approach to promoting patient safety should be made clear to all stakeholders. Patient-oriented process optimization and patient satisfaction are the focus. In addition, quality management should help increase the satisfaction of everyone involved in the process. " (p. 4 translated by the author)

Quality management includes in particular the so-called basic elements (G-BA, 2016)§ 3 - translated by author, italics by the author):

- Patient orientation including patient safety

- Employee orientation including employee safety

- Process orientation 
- Communication and cooperation

- Information security and privacy

- Responsibility and leadership

In the context of this study, it should be emphasized that the QM guidelines in their version valid since November 2016 have recently explicitly called for the inclusion of the employee perspective, as incorporated in the MFA survey and the decision model: "Systematically integrating the employee perspective - In addition to regular patient surveys, in the future also employees - if possible anonymous - to be interviewed. This gives the practice management suggestions for changes and potential for improvement "- (KVB, 2017 - translated by the author).

Even before the further development of the QM guidelines, "The Holistic" practiced quality management with a focus on patient and employee orientation, as the following explanations vividly demonstrate: “... The MFAs discuss a lot during routine routines. It is advisable to do sports, talk about healthy nutrition, get into yoga, etc. tells us that we also do yoga for the team. But that's just as individual as my consultation. We pay close attention to who, how and when it fits. A homebound elderly lady will expect something different than a 22-year-old young man. Just as there is no "relaxation process" for everyone, it is necessary to pick the individual where he is. And so, for one patient, this may be newspaper reading or sudoku games, while for another, the walk, the church choir, meditation, autogenic training can be used to prevent stress. I already had MFAs who were very involved. One member of staff has trained in metabolic balancing and offered regular one-on-one talks and courses. Currently, a former employee at weekend offers balanced nutrition courses. We have offered courses on diapers and pads. One patient offers yoga in our rooms 3 times a week. I let the staff come. If an MFA is interested, it will be very supportive, ..." (translated by the author)

The relationship between the proposed primary prevention measures and the quality of the practice is initially not obvious and directly identifiable. But Gavartina (2014, p.2) states in a survey of 2,000 specialists: "Ensuring medical care depends on both the general practitioner and the MFA and can be significantly influenced by the perception of satisfaction factors and the organization of care. " (Translated by the author). Szecsenyi et al. (2011) can provide statistically considerable evidence that the MFAs satisfaction is decisive: "However, the correlation between the job satisfaction [of the physician] and the patient satisfaction is lower and not significant. Interestingly, the job satisfaction of the non-physicians seems to be more associated with patient satisfaction than physician satisfaction. " $(\mathrm{N}=676$ primary care practices in Germany). Vu-Eickmann and Loerbroks (2017, p.4) conclude that working conditions and lack of social recognition have an impact on MFA job satisfaction as a result of 26 qualitative interviews, which is a key determinant of patient care quality. Ultimately, if the general practitioners give "their" MFAs health management services, it is also an act of social recognition and appreciation of their work. Stressful 
and dissatisfied employees make more mistakes, as noted by Gehring and Schwappach (2014) in their study of 630 doctors and MFAs in German-Swiss medical practices.

These and other contexts are included in the Rhodes and Steers (1978) model adapted for physician's office in subsection 3.1.3 "Applying the model to general practitioners' surgeries". Although it is primarily about attendance or absence, the illustrated, fundamental relationships between job satisfaction and motivation can be successfully transferred to the quality management in general practitioners' surgeries.

The proposed measures contribute both to patient orientation and safety and to employee orientation. The latter also because they consider the preferences and the employee in his individuality. In particular, when the "health on their own" by the MFAs in the specifically requested team discussions (G-BA, 2016), a starting point could be set here, and then later tackle the action priorities "work organization" and "leadership quality" identified in the target system.

"Quality management supports the practice management and the team in structuring work processes, defining responsibilities and identifying risks at an early stage. The aim is to align all activities consistently with professional, legal and contractual fundamentals - and to orient themselves as close as possible to the needs of patients and employees." KBV (2015- translated by the author)

In this research, a decision-making procedure on "health management" was presented on selected primary prevention measures. The presented decision criteria (acceptance, effectiveness and cost level) as well as the associated multicriteria decision-making model can be transferred to other explicitly mentioned application areas of quality management in the medical practice:

- Complaint management

- Risk management

- Interface management

- Error management

- Emergency management

- Hygiene management

\subsection{Bridging the implementation gap}

Before the implementation gap at the introduction of the priority list for primary prevention is discussed, the importance of primary prevention for the German general practitioners' surgeries is to be made clear first. This is necessary to work out the terms of a successful implementation. 


\subsubsection{The relevance of primary prevention for MFAs}

The delegation of medical activities to qualified non-medical professionals such as MFAs is often seen as a solution to the problem of the shortage of physicians and the unequal distribution of urban and rural areas. Dini, Heintze et al. (2012) investigated in their project AGnES (community-based, e-health-assisted, systemic intervention to reduce physicians' workloads) for the German federal state MecklenburgVorpommern possible relief effects due to the fulfilment of home visits by the MFAs. In the preventive area, more than $60 \%$ of the general practitioners $(n=515)$ rated fall prophylaxis, vaccinations and nutrition advice as important. Although this result is not representative for the whole of Germany, since MecklenburgVorpommern is a typical area state, it shows, as already noted by Regus (2012), that measures to promote personal health potential, especially in the case of MFAs, are not only have a lifestyle aspect, but also a high importance in job and work design.

The final report on a study in the metropolitan state of Nordrhein-Westfalen (Dini, Hirth et al., 2018), shows a similar result in the field of diagnostics, which is based on the "survey of vaccination and smoking status and current drug therapy (incl. selfmedication)" as a task with a high delegation potential. Only $7 \%$ of the 724 general practitioners responding consider this task to be undelegated, and the potential for expansion is classified as "very high". The implementation of the vaccination itself is part of the task "medication of intramuscularly injections" into the field of therapy and treatment and is considered by $11 \%$ of the responding family doctors $(n=723)$ as non-delegable. In the field of training and counselling, the task "supporting patients with necessary lifestyle changes (nutrition, exercise, social contacts)" is classified as non-delegable by almost $12 \%$ of general practitioners $(n=702)$, the expansion potential is also considered "very high" here.

While the focus for the above studies is on the physician's relief by delegation to the MFA, the project "Competence Development of Health Professionals in the Context of Lifelong Learning (KeGL)" is about a reorientation of the professional profile of the MFAs (Babitsch and von Moeller, 2017), which includes the delegation, but which focuses on a broader competence aspect and corresponding training modules. "From the point of view of the 43 experts surveyed, the development of new forms of supply (larger, more complex units), changes within the supply areas and the emergence of new interfaces between changes across occupational groups relevant to outpatient, inpatient and public health care for those working in pharmacies and doctor's and dental practices. In addition to adapting to scientific advances in medicine, dentistry, pharmacy and technology, the professional groups MFA, ZFA, PCA and PTA are responsible for new areas of activity and segments, the redistribution of activities within the teams and new forms of cooperation with other health professions. In addition, new forms of communication and increasing consulting activities are required. From an expert's point of view, aspects related to patient safety, delegation, technology and case orientation as well as interfaces with other health professions represent occupational changes for the MFA occupational group." (Babitsch and von 
Moeller, 2017, p. 8 - translated by the author). The MFAs surveyed $(n=67)$ see the increasing importance of prevention/health promotion and recognize that patient education (information, training and counselling) is also playing an increasingly important role. "Prioritizing the results of the expert interviews and the employees around the employer survey led to the identification of occupational and crossoccupational topics on which the initiation of further or new competences is desirable. Prevention and health promotion are one such area." (Babitsch and von Moeller, 2017, p. 11 - translated by the author).

\subsubsection{Content-oriented and process-oriented perspectives on implementation}

Summarizing the Literature review on the implementation gap from section 3.2.6, two levels can be distinguished. On the one hand, the content level, which deals with the question of "what should be implemented", and on the other hand the processual level, which revolves around the question of "how is implemented and how is this perceived".

The proposed introduction notes on the personal health promoting activities and the adaptation possibilities of the standard proposal, as well as its situation-specific integration possibilities into a quality management concept discuss to a large extent the content aspect of the implementation. The importance of primary prevention in a changing field of tasks and occupations, as described in the previous paragraphs, must also be attributed to the content level. Standardization, decision-making and prioritization play an important role for a successful implementation for this level.

Bowen and Ostroff (2004) criticized the one-sided focus on the content-based approach, where researchers take into account the inherent virtues (or vices) associated with the content of HR practices to explain performance (Sanders et al. 2014, p. 489). While the content-based approach focuses more on individual HR practice in order to achieve a specific goal, the process-oriented approach focuses on creating strong situations in the form of shared meaning about the content, which ultimately performance increase. The process-oriented approach to HRM highlights the importance of the psychological processes through which employees interpret and respond to the information conveyed in HR practices (Bowen and Ostroff (2004); Ehrnrooth and Björkman (2012) - cited in Piening, Baluch et al. (2014, p. 546)).

This puts the psychological contract in the spotlight, which for the general practitioner's surgery mainly represents the process-oriented aspect. If you follow Sanders et al. (2014, p. 492), an implementation challenge is to make the original intention of the key stakeholder as unadulterated as possible and unchanged to the employees. The work of Nishii et al. (2008) has highlighted the role of key stakeholders, especially the employee's immediate manager. Following this line of reasoning, the question of what roles and behaviours line managers should perform in order to signal concern for well-being is receiving growing attention (Shipton, Sanders et al., 2015). Since in the general practitioner's surgery the key stakeholder is also the immediate 
manager and holder of the psychological contract at the same time, no friction alder is to be feared in this respect.

\subsubsection{The psychological contract and leadership ability}

However, this gives the person and personality of the physician and his or her capability to fulfil the psychological contract and the associated leadership ability a high relevance. This coincides with the cross-impact matrix derived from literature review, in which leadership ability was identified as one of the strongest impact factors (see Table 2).

The employee's question about the "why" (Nishii, 2008) or, more generally, "sensegiving", raises an aspect of the implementation process that, although not a direct part of the psychological contract, has a lasting effect on its fulfilment. It is the question of the credibility of the employer and his/her motivations associated with the introduction. Shipton et al. (2015) found that where employees perceived that HR practices were implemented with an eye to their needs and aspirations, they were more inclined to report positive attitudes and to behave in a way conducive to the achievement of organisational goals. Nishii et al. (2008) draw attention to the fact that the success of health and well-being measures depends not so much on their content, but on the employer's management-philosophy and the motivation behind recognized by the employee.

Scharf, Vu-Eickmann et al. (2019) surveyed 887 employed MFAs between September 2016 and April 2017. A 20-item questionnaire measured desired improvements. Those desired improvements, which were closely related to leadership ability were among the most common mentions: "I would like the physicians to have educational opportunities related to organizational leadership" (75.1\%). - "I wish for more appreciation for my work from my supervisor" (60.8\%) - "I wish for more understanding by my supervisor" $(60.3 \%)$. This reveals possible difficulties in the implementation with regard to the relational promises to fulfil the psychological contract.

The same study also examined the transactional aspect of performance-based remuneration, which is considered by Atkinson (2005), Millward and Brewerton (1999), and Pate, Martin et al. (2003) as hygiene factor and precondition for the fulfilment of relational promises. "I would like to have a higher salary" was with 759 mentions (87\%) the most expressed desire for improvement. Scharf et al. (2019a) explain: The issue of a low salary remains a crucial aspect, nonetheless. The average monthly gross salary of fulltime employees in the service sector in Germany has amounted to 3,719€ in 2017 (Statistisches Bundesamt, 2018). MFAs usually receive a gross salary far below that average. In our study, for instance, only 58.2\% of MFAs working fulltime reported to receive a gross salary of at least 2,000€. Close associations of low income with poor health have repeatedly been documented (Wood et al., 2012; Martikainen et al., 2003; Lynch and Kaplan, 1997) and should be considered when addressing needs to improve the working life of MFAs. 
In a second evaluation, Scharf, Loerbroks et al. (2019) note that needs pertaining to working conditions and reward from the supervisor were the strongest determinants of MFAs' consideration of leaving their employer or profession. The difficulties resulting from fulfilling the relational promise of "a pleasant and safe working environment" can be described as follows: While MFAs are dissatisfied with many of their working conditions (e.g., salary, multitasking), it seems that they perceive very limited opportunities to change those conditions (Vu-Eickmann et al., 2017). If they would want to talk about improving their working conditions, MFAs would need to contact (and potentially criticize) their supervisor, since there are no employee representatives in small outpatient practices with few MFAs (Vu-Eickmann et al., 2018). In outpatient practices, the direct supervisors of MFAs are commonly physicians who employ the MFA and on whom they are thus financially dependent. Therefore, most MFAs dissatisfied with their working conditions report to primarily pursue individual-level strategies, which mainly pertain to exit strategies and thus the intention to leave the employer or the intention to leave the profession (Kathmann and Dingeldey, 2014; Vu-Eickmann et al., 2018).

In his socio-technical system analysis and evaluation of medical practices, Majumdar (2004) points to other fundamental problems which, in the author's view and experience, make it difficult to fulfil the psychological contract and thus constitute a cause of the implementation gap should be considered:

- Management and management skills have not been systematically taught and are not sufficiently imparted in training and education of general practitioners. The general practitioners can have a preventive effect on many possible problems if they are conscious and targeted of the optimal work structures, conditions and priorities for them (e.g. practice form, more collegial exchange, unique selling points, etc.) This applies in particular regarding the psychological contract, the fulfilment of which is a prerequisite for the necessary commitment of the employees.

- The central importance of personal competences, especially in the form of selfmanagement competence, has so far been little or no account taken in the medical qualification phase.

- Management and management tasks are often perceived as at odds with one's own professional self-image. Following Ulich (1981) in the context of a psychologically qualified approach, it is necessary to take them seriously and to support the general practitioner in developing appropriate awareness of the problem and in taking a conscious point of view, which then forms the basis for truly "wanted", targeted and self-motivated action within the framework of quality management.

- The particularly close, reciprocal relationships between professional (including cooperation with MFAs and dealing with patients) and non-professional (including life partners) tasks and areas of private life require a high level of self-competence to identify "blind spots". 
- Self-management as part of the leadership ability is diagnostically and intervening as an essential "leverage" in the context of management development in ownercentred small businesses.

This assessment of the importance of leadership ability has been reinforced by the qualitative interviews with general practitioners on the preference profiles. Although the survey is not representative, the fact that only one general practitioner interviewed put the question of the importance of leadership ability first deserves to be taken. Significantly, this assessment came from the physician, who corresponded to the "Experienced" profile.

\subsection{Summary of results}

The following points summarize the research findings of the chapter "discussions"

They contain three approaches to introduce the proposed measures and thus contribute to knowledge for the area "Implementing primary prevention measures in German general practitioners' surgeries".

Moreover, the research questions

4. How can the procedure of decision-making be adapted to the specific conditions of an individual general practitioner surgery?

5. What approaches should the general practitioner (as owner) consider if he/she wishes to set priorities for the introduction of a health management system?

are answered:

- The procedure of decision-making can be adapted:

- by the design of the proposed measure

- to the age structure of the staff

- to the weighting of the decision criteria

- by adding own measures

- As part of the "standard proposal" introductory approach, general practitioners should prioritize the three measures "Vaccination", "Healthy snack at work" and "Training at home".

- To use the list of suggestions as a starting point for the approach "health management", the general practitioner should review beyond the measures for personal health promotion, her/his target system for "work organization", "job design" and "leadership ability". 
- To extend the approach of "health management" to the approach "quality management", the target system should be focused on "patient orientation" and extended by the elements "process orientation", "information security and privacy" as well as "communication and cooperation".

- For a process-oriented approach to the implementation of health management systems, the need for leadership ability of general practitioners was developed as a prerequisite for fulfilling the psychological contract. 


\section{Conclusions}

This research aims to improve the knowledge in health management at general practitioners' surgeries in Germany with a focus on activities promoting the employees' health potentials. The previous chapters contain a great deal of information and evidence in this respect. This last chapter highlights the salient points that may be used for drawing conclusions and which indicate the possible contributions of this study. The contributions relate to empirical research, methodology and practical application. The limitations of the research undertaken, and possible future research questions based on the outcomes are also considered in this chapter.

Since this chapter is a summary, the literature sources are not specified in detail except for key results. Rather, it should be referred to the respective sections and passages in the previous chapters.

\subsection{Contribution to empirical research}

The overall aim of this research was to develop a concept to support stakeholders in promoting health supporting activities which facilitate personal health potentials and help improve the performance of German general practitioners' surgeries.

The development of such a concept affects different areas of research. On the one hand, there is health management itself, which includes measures to promote personal health potential. Closely associated with this are HRM theories that examine the employee-related connections between HR practices and organizational performance. Since German general practitioners' surgeries are usually micro-enterprises, research results of the small firm theories had to be considered as far as possible. The pragmatist approach taken in this DBA-thesis points to an area that examines the interface between theory and practice and whose inadequate support for theory is often described as an "implementation gap".

The literature review found that the following areas can be considered as little explored (under-researched):

- Health management in general practitioners' surgeries

- Human resource management in micro-enterprises

- Psychological contract in micro-enterprises

- Implementation of health management measures

In the following it is shown, which contribution this work contributes to the closing of the research gaps and which further implications can be drawn out. As a pragmatist, the researcher is faced with the fact that there are no theories relevant to health management in micro-enterprises or to the management of general practitioners' surgeries - let alone that they are also classified as robust and data-driven. As far as possible, individual aspects of the theories were substantiated by empirical studies on the MFA working environment, which were then integrated into a more comprehen- 
sive framework in combination with the personal contract, which was considered appropriate for the situation.

\subsubsection{Health management in general practitioners' surgeries}

As a contribution to health management, the author developed a target system for health management in general practitioners' surgeries based on a proposal by Walter (2007). Adapted to the circumstances of a micro-enterprise, it includes the goals of increasing leadership ability, strengthening social and human capital, improving health and well-being and improving performance, quality and economic efficiency. Within the area strengthening social and human capital, the sub-goals of healthpromoting work design and work organisation and personal health potential (prevention) has been identified. The sub-goal for general practitioners' surgeries prevention was determined as having the highest potential for action. To give this sub-goal a particularly high priority is justified by the special role and importance of prevention measures in the professional field of MFAs. Prevention is one of the most likely to be delegated by the general practitioner. If an MFA can gain personal experience with preventive measures, this suggests that she can also contribute them to the patient relationship in a successful and competent manner.

As a further contribution to health management, activities that promote personal health potential and help prevent illnesses in the workplace were identified in a systematic literature research. For them, it was examined which peer reviewed articles document a reduction of absence. Vaccination programmes, programmes for activity promotion (5 different measures) and activities for weight reduction and change of nutrition (4 measures) met the selection criteria.

The success of these proposed activities essentially depends on the acceptance by the employees. In general practitioners' surgeries, measures to promote health are addressed to the MFAs. In a survey, MFAs of general practitioners' surgeries were asked about their preferences for primary prevention measures. Compared to other occupations, this occupational group has an extremely high proportion of women. The age structure with two local maxima (instead of one) is atypical. The evaluation of the MFA survey provided a comprehensive preference profile for primary prevention. Both willingness to take part in measures and experiences with healthpromoting activities were examined. The acceptance was evaluated for ten different prevention measures in the fields of vaccination, nutrition and movement. Age and experience-related differences were examined by hypothesis testing for statistical significance.

Possible combinations of measures were evaluated. The most preferred combination with a movement and a nutritional program is "Sponsored activities (Fitness, Yoga...)" with "Healthy snacks/food at work". The least coveted combination of measures is "Training within a group of colleagues" with "Internal nutrition consult- 
ing with a physician from the medical office." For almost $90 \%$ of the MFAs, at least one combination was attractive.

When surveying MFAs according to their preferences, data were also collected to assess whether general practitioners' surgeries problems are to be expected as a way of demographic change. The comparison of the age distribution of the MFAs surveyed with the average profiles of female German employees and employees in health professions clearly shows that the challenges for general practitioners' surgeries are significantly lower.

Acceptance is not the only criterion used to decide on the introduction of preventive measures in general practitioners' surgeries. There are also other criteria such as effectiveness and cost level. Assessment methods have been proposed for these criteria. For prioritization, a multi-criteria decision model was developed and applied. The result, the priority list, was subjected to a sensitivity analysis to examine how robust the model is against changing criteria weights.

The assumption that MFAs favour cost-intensive or particularly effective measures was also investigated. The hypothesis test revealed: MFAs do not favour the costlier individual programs for health promotion. The hypothesis, MFAs prefer the more effective of the individual programs for the promotion of health, cannot be rejected or confirmed.

The hypothesis that expensive measures have a higher effectiveness and vice versa was tested, too. It must be dismissed, so that the counterhypothesis applies: Cost level and effectivity of the individual programs for health promotion are in-dependent (i.e., the two features are not correlated strong).

The promotion of personal health potentials (primary prevention) is only a sub-goal of health management in general practitioners' surgeries, which can be assessed in terms of acceptance, cost level and effectiveness. The same can be achieved for the other sub-goals such as leadership quality, psychosocial well-being, or risk factors. As further implication, a system can be set up that compares the status of health management in individual general practitioners' surgeries and can be integrated into more comprehensive quality management systems. To promote the achievement of goals, a pool of best practices can be built up.

\subsubsection{Human resource management}

As Peccei et al. (2013) point out, the theoretical and empirical picture of the processes and mechanisms that combine HR practices, health and well-being with organizational performance is very complex. On the one hand, there is the fundamental theoretical question of whether health-promoting measures only do not contribute to the increase in performance but can also be counterproductive under certain circumstances. On the other hand, a large number of contingency and context factors make 
it difficult to develop a coherent overall model. This work addresses the topic of health management and human resource management for the first time.

To answer the question of whether health and well-being is more likely to cause conflicting outcomes or mutual gains in terms of performance in German general practitioners' surgeries, this study contributes by examining two work science models. Within these models wherever possible, evidence is substantiated by empirical studies. These models are the general process model of attendance (Rhodes and Steers, 1978) and the causal model of absenteeism (Brooke, 1986).

For the general process model of attendance (Rhodes and Steers, 1978), the author examined whether this process model can also be applied to the circumstances of a general practitioner's surgery. This research proves that the variables identified in the general process model, which have an influence on the presence and absence, can be applied to the work situation of MFAs in general practitioners' surgeries. It has been demonstrated that taking on primary prevention tasks by MFAs, which are closely related to their own primary prevention, offering the opportunity to improve the variable "job situation". For the variable "employee value and job expectations", low recognition and low income have been identified as factors influencing attendance. The high part-time rate and the associated permanent work-privacy conflict affect the variable "satisfaction with job situation". The variable "pressure to attend" is characterized by the balance between work ethic, loyalty to the (small) team and the economic pressure. In addition to these variables influencing motivation over the long term, the factor illness was identified as a short-term effective of the variable "ability to attend". This factor, in turn, is influenced by primary prevention, the subject of this research. Overall, the general process model of attendance (Rhodes and Steers, 1978) suggests that mutual gains will occur, as the expected increase in competence and takeover of new tasks, for example in patient counselling and care, will increase motivation and reduces absenteeism and thus increases performance.

The causal model of absenteeism (Brooke, 1986) is used to investigate and analyse the direct (endogenous) and indirect (exogenous) influencing factors on absenteeism. Apart from "alcohol involvement", absence is essentially caused by the endogenous factors of "satisfaction", "job involvement" and "commitment", as well as "health status”. For general practitioners' surgeries, all empirical studies were evaluated, which prove an effect on the endogenous factors affecting the exogenous factors. It becomes clear that primary prevention and the possible expansion of the range of tasks can also be accompanied by conflict outcomes. Taking on new tasks can lead to "overload", "role conflicts" and "role ambiguity", which in turn directly burden health status and are exacerbated and amplified by dissatisfaction.

One contribution of this DBA-thesis to empirical research into the link between HR practices and organisation performance is to find that there are so many contingency factors in the German general practitioners' surgeries that none of the HR theories have an adequate systematic model of impact and explanation. As contingency fac- 
tors which, despite the homogeneity of the general practitioners' surgeries, prevent to include the embedding in a theory, were found: high proportion of part-time employees, extremely high proportion of female employees, atypical age pyramid, lack of role of trade unions and professional associations, classification of a doctor's office as a service and business enterprise, permanent structural changes in the public health system, regulated market with unclear competitive conditions and the size of the organisation as a micro-enterprise.

\subsubsection{Small-, medium-, and micro-enterprises}

The size of an organization is not just a contingency factor. It is inversely proportional to the number of researches (and inversely proportional to the economic importance).

A first promising approach to the formation of an HRM model for general practitioners' surgeries was found in the application of the psychological contract for "Building high performance employment relationships in small firms" (Atkinson, 2007). From differing industries, manufacturing, financial services and information technology, and company sizes, it is shown that it is possible to develop a psychological contract based on cognitive and affective trust and on distributive and interactional justice, which is to be performance-enhancing results. The underlying empirical research (Atkinson, 2005) is based on SMEs with 65 to 90 employees. Even for SMEs with a much larger number of employees, Atkinson (2005) argues there is no need to make distinctions in terms of the content of the contract.

The author sees it as a further contribution to empirical research that after examination and follow-up of the arguments for the psychological contract, the presented results are not only for larger enterprises applicable, but also for micro-enterprises as it is general practitioners' surgeries with 1-5 employees. According to this, expected performance increases are not solely dependent on a specific HR practice, but on the mutual fulfilment of the psychological contract by the employer and the employee. From this point of view, the employee attitudes, motivation from the process model for attendance (Rhodes and Steers, 1978) and satisfaction and commitment from the causal model for absenteeism (Brooke, 1986) are to be supplemented by the level of trust.

As a further contribution to empirical research specifically for general practitioners' surgeries, the author proposes an extension and adaptation of an operational model proposed by Katou (2013), that is linking HR practices, the psychological contract and the organizational performance. It is assumed that the state of the psychological contract and related changes control and mediate the relationship between HR practices and organizational performance. The fulfilment of transactional and relational promises by the employer leads to attitudes of the employees, which enable and promote the fulfilment of the transactional and relational promises for their part. The 
factors influencing the promises are based on the best practice proposals of the CIPD fact sheets (2008).

Although this model also has only been validated for SMEs with more than 20 employees using factor-analytical methods, the considerations and discussions made in this DBA-thesis suggest that it also suitable to be a framework for research. The figure below shows the model in its further development and adaptation for general practitioners' surgeries. Under HR Practices such ones have been included, which, according to an expert survey, will be of high or increasing importance for MFAs in the future. As a reference for Organizational Performance, in addition to Efficiency and Effectiveness, the basic elements of the QM Guidelines (GB-A, 2016), which are mandatory for doctors, were included as indicators. The Employee Attitudes were supplemented by Trust and italicized, as were the other points Personal health promotion, Employee orientation and Leadership that were specifically examined in this research.

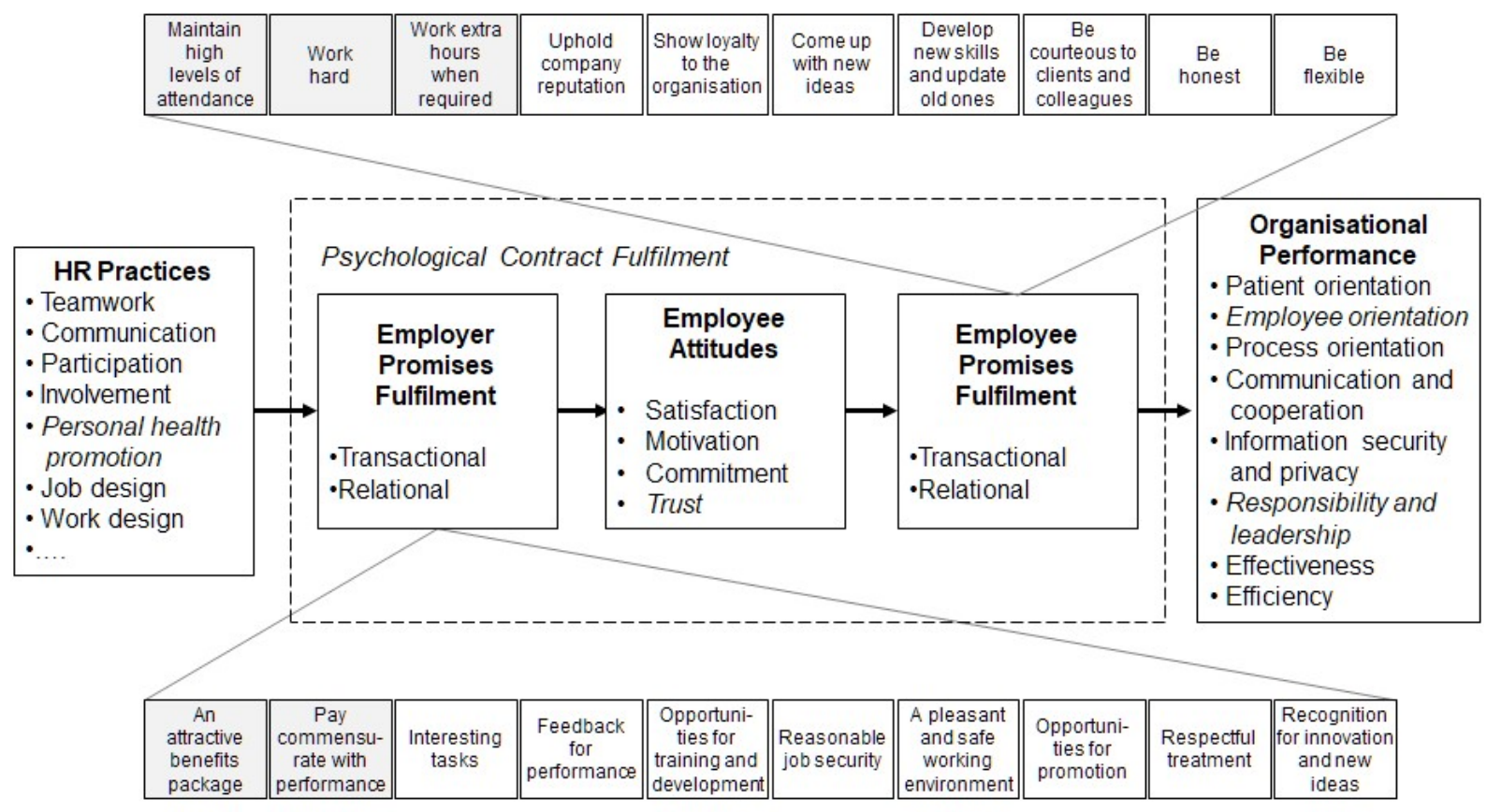

Figure 46: Operational HRM-Model for German general practitioners' surgeries (source: Katou (2013) - supplements by the author)

In addition to the research purpose, the framework can serve as a training and education reference model for general practitioners, providing a holistic framework for teaching management and leadership skills. It leaves room for different leadership styles and enables the performance alignment of the individual practice. It familiarizes the physician as an employer with the expectations addressed to him/her. In the MFA vocational training, it can be used to clarify the overall context and at the same time clarifies the expectations MFAs can have on the physician as an employer and what can be expected of her. 


\subsubsection{Implementation gap}

The operational model presented above accomplishes a contribution to bridge the implementation gap, since it brings together the content perspective of an HR practice with the process perspective inherent in the psychological contract.

In terms of content, the contribution is made via empirical reasoned standardization. This concerns both the target system for health management in general practitioners' surgeries and the preference profile of the MFAs and the three implementation concepts developed for the implementation of the priority list with the proposed measures to promote the personal health potentials. For SMEs and micro-enterprises, the deployment of empirically justified, theoretically sound and, if necessary, adaptable standardizations is of great importance due to the time and induction efforts.

The contribution of a better understanding of the psychological contract is essentially to be seen in the fact that it focuses the socio-psychological aspect of the implementation from the very beginning. Also, content-based and proven concepts can only be implemented in a targeted manner and perceived as successful if they are presented credibly and meet with mutual trust. This is the only way to use commitment, satisfaction and motivation to achieve the performance improvement hoped for by the respective HR practice.

In this context, a difference in the psychological contract between SMEs and microenterprises such as general practitioners' surgeries should be noted. The relationship between the physician and the MFA is very close. Both are in daily, direct contact and have numerous opportunities to communicate goals, intentions, expectations and perceptions. In larger organizations, there is almost always only indirect contact, which takes place over several stages. Each stage carries the risk that the original goals, intentions and expectations of the "owner" will be distorted by intermediary interaction and communication processes and will not be preserved and perceived in their originality.

This brings to the fore another aspect as a contribution, the physician's self-image as a manager and her/his leadership ability. Empirical findings for the leadership of general practitioners are scarcely available. Majumdar (2004) investigated the need for this qualification and stated, that it is not the subject of medical studies. The doctor is educated as a specialist and not as a leader.

This is in line with the findings of the literature review, which examined which control levers are most effective for the implementation of a health management system in general practitioners' surgeries. Thus, the promotion of personal health potential, the subject matter and contribution of this DBA-thesis, is most important, followed by the improvement of the leadership ability of general practitioners and a health enhancing job design. 


\subsection{Contribution to methodology}

From a methodological point of view, this study was carried out with a two-stage research design that follows a pragmatic philosophy. The first stage was carried out using a quantitative method as an MFA survey. Its results were incorporated into a broader qualitative oriented decision-making process to prioritize prevention measures, which was reflected in qualitative interviews with general practitioners in the second stage. The author feels committed to pragmatism and accordingly concepts, theories and models become particularly relevant if they have a high degree of relevance to the general practitioners' surgeries in their consequences.

As a pragmatist, the author acknowledges that there are manifold ways to interpret excerpts of reality and conduct research. Different perspectives lead to different perspectives of the same reality section, which in turn lead to different decisions with possible options for action. To show how these different perspectives of different stakeholders can be handled in a systematic (and thus interactional and distributive justice) decision-making process and implemented in a goal-oriented list of priorities, is as a result the author's main contribution to methodology. The overall concept as used throughout in the development of the priority list is shown in the following figure.

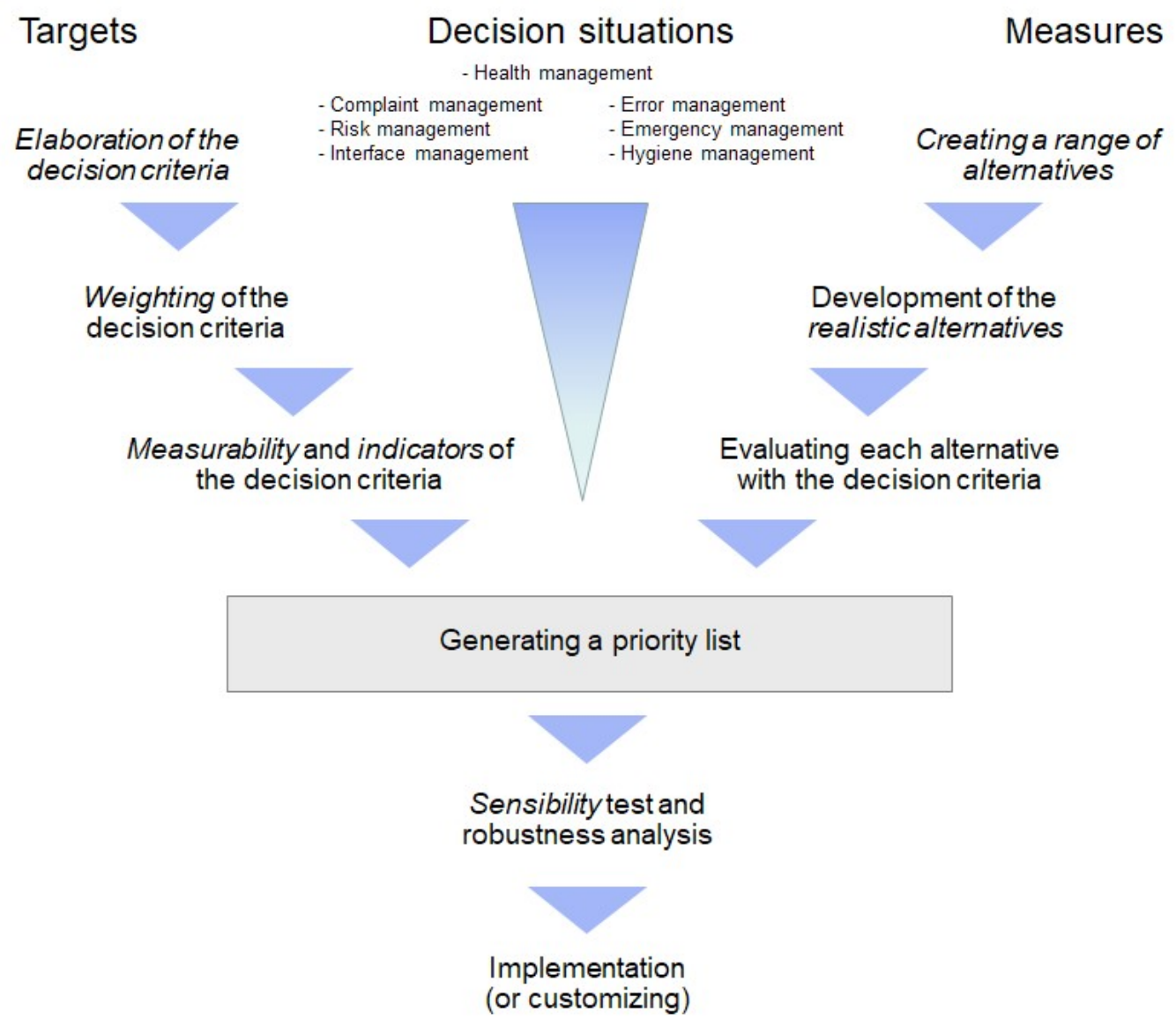

Figure 47: The multi-criteria decision-making model for German general practitioners'surgeries 
For measures to promote the personal health potential of MFAs, the different perspectives of acceptance, effectiveness and cost level were identified, which then find their way into the decision-making method as decision criteria in the Targets branch. For further development, they must then be weighted in consultation with stakeholders and provided with possible indicators for evaluation.

For the Measures branch, the first step is to identify as many alternatives as possible that could contribute. In order to ensure manageability, the realistic alternatives will then be filtered out, if possible, also in consultation with the stakeholders. In the case of health management in general practitioners' practice, the initial more than 25,000 articles in relevant databases were restricted into a drill-down process to those for which peer-reviewed articles demonstrated the efficiency of reducing absence was documented. Finally, ten preventive measures for MFAs were shortlisted after the exclusion of further measures which are more likely to be classified as individual medical treatment (e.g. alcohol and other addiction problems) and then an evaluation according to the criteria of acceptance, effectiveness and cost level was carried out.

Acceptance was assessed according to the preference profile, and the peer-reviewed articles were used to assess effectiveness. The cost level was estimated based on practical values in a pair-by-case comparison.

A prioritizing concept is, on closer inspection, a ranking problem in which the different perceptions of the stakeholders are placed in a comprehensible order. One method of solving ranking problems is multi-criteria decision analysis (MCDA). This method was developed to provide answers to complex questions with several divergent stakeholder interests (Ishizaka and Nemery, 2013). The idea of MCDA is not to find the one correct decision, which solves the problem, but the results will be a list with all possible activities ordered by priorities. The priority list generated with the M-MACBETH software (Bana e Costa, De Corte et al., 2014) shows a ranking of all measures considering all three decision criteria, thus presenting the most favourable activities (see Figure 36). The results were then also used with M-MACBETH software executing a sensitivity analysis to determine the extent to which deviations in assessment and weighting by the stakeholder perspectives lead to a different ranking of priorities. The outcomes were included as a contribution to empirical research.

In the second stage of this research, the manageability and completeness of the decision model was examined in structured interviews. This revealed that the average of the weighting of the decision criteria acceptance, effectiveness and cost level in the structured interviews does not deviate significantly from the proposed weightings in the priority list.

From a methodological point of view, the proposed decision-making model can be seen as part of the operating model (Figure 46) as a participatory model for negotiating the psychological contract. For the employer, the understanding of the employ- 
ees' preferences becomes clearer, and the latter, in turn, will appreciate that not only their preferences are considered, but that their interests are also considered in the weighting and evaluation of the decision criteria. It seems obvious that satisfaction, motivation, commitment and level of trust improve promisingly.

As a method for bridging the implementation gap, it can make a valuable contribution, especially if, as described above, it has been used as a participatory model of action, as mutual knowledge of stakeholder perspectives has an interest-balancing effect.

The decision-making model applied in this research, uses the acceptance by employees, cost level and professional effectiveness as decision criteria. These reflect the perspectives of internal stakeholders. As an increasingly important perspective of public stakeholders, which should be taken into account in all decisions, the "CO2 resource consumption" could be included in the decision-making process as an additional standardized decision criterion. At first glance, this does not seem to be important in primary prevention measures. If one considers that, for example, individual trips to the fitness centre mean significantly more $\mathrm{CO} 2$ equivalents than group training at the workplace or that different diets can lead to significantly different ecological burdens, it quickly becomes clear that even micro-enterprises can, or should, incorporate environmental considerations in their decisions. This criterion would then be conceivable, for example, a weight of $10 \%$. As an indicator, the author would recommend the so-called "Ecological Footprint", which is increasingly available for various activities. According to the methodology, the weighting should be checked for robustness by means of sensitivity analyses.

As a further contribution to methodology, the cross-impact matrix practiced by the author and designed for this purpose will be seen. In a given target system where sub-targets can reinforce each other, can be conflicting with each other, or have no dependencies, a cross-impact matrix can, following Zangemeister (2014) be used to identify the target that is the first to be addressed. The interdependencies between two targets are examined based on theoretical and empirical models and are recorded weighted according to the strength of the relationship. In this DBA thesis, the method for the target system of health management in the general practitioners' surgeries was developed and resulted in the conclusion that primary prevention, leadership ability and job design come first, as they have a particularly high impact on the other targets.

\subsection{Contribution to practice}

In developing an operating model (Figure 46) based on a framework with two systems (Katou, 2013) adopted by HR professionals, the psychological contract system and the HR practices system, the author suggests that the model presented in this DBA thesis may contribute to HR practice. The developed decision-making model 
for the priority list (Figure 47) also contributes to HR practice by using it at the interface of theory and practice to close the implementation gap.

There is no Human Resource Management concept for German general practitioners, so this work is breaking new ground. With the preference profiles, the prepared measures (standard proposal) and the implementation recommendations, the practical doctors are provided with instruments that facilitate practice management and save valuable time of the general practitioners as it was confirmed in the qualified interviews.

The benefit and value of the framework beyond the time saved results from the possibility of using the framework to reflect the link between HR practice and performance. The impulse to see the framework of one's own surgery in the categories of the psychological contract helps to improve one's own management skills, which are not part of the training as a general practitioner. The mutual fulfilment of the psychological contract as an indispensable prerequisite for the implementation of any HR practice - and health management is just one of many recommended - to recognize ensures the sustainable success. This becomes all the more important as the cost pressure and the quality requirements increase. Thus, not only the medical professional qualification, but also the motivation, satisfaction and commitment of the MFA team becomes more important.

Another benefit of the framework is the ability to use the indicators for fulfilling the psychological contract to develop a profile of strengths and weaknesses for one's own surgery and thus position oneself in the competition. First, in comparison with other general practitioners' surgeries, but also in comparison with the competitors mentioned in sub-section 2.2.3: Group practices and practice sharing, specialist surgeries, or medical care centres. If general practice is aimed at a high proportion of lucrative private patients, the indicators and the framework also offer the opportunity to put one's own practice to the test.

The decision-making model (Figure 47) shows the structure of the model and at the same time identifies comparable decision situations from the quality management of a physician's practice, in which, in the author's opinion, the model could be used equally. For example, in hygiene management, the permanent compliance with disinfection measures can only be guaranteed if they are accepted and internalized by the MFAs. The assessment of effectiveness, like primary prevention, is based on the expertise of the physician and the MFAs, as well as on relevant studies, literature reviews and guidelines from the medical associations and professional organizations. For example, in order to increase effectiveness, the medical associations recommend not only suitable emergency equipment as part of emergency management, but also regular team training. The third criterion, the cost level, could be estimated like that of primary prevention. The main cost factor is the time spent. If, for example, complaint management complies with the rule that "complaints are whenever possible, a 
matter for the boss", this is more cost-intensive than the friendly handling of patients, in order to counter the complaints in advance.

A further benefit and contribution to HR practice is seen in the fact that the developed models can be adapted to other situations and questions, i.e. have a generic character, which is briefly outlined below.

\subsubsection{Adaptation to individual stakeholder realities}

For the multi-criteria decision-making model developed, the options for adapting own measures, changing weightings, considering the age structure and selecting possible individual measures were explained. Without much effort, the multi-criteria decision-making model can be flexibly adapted to individual circumstances, both in terms of criteria and measures. In addition to the decision criteria acceptance, effectiveness and cost level, further decision criteria can be included. Likewise, the weights can be changed and the indicators for the measurability of the criteria. The proposed ten measures can be replaced or supplemented by own proposals. This allows general practitioners to create a list of priorities tailored to their own needs.

\subsubsection{Specializations of medical practices with MFAs}

A first approach to the transfer of outcomes would be the specialists from different medical disciplines, who also employ MFAs. In square brackets the number of physicians according to the statistics of the Medical Chamber (2017).

Paediatrician practices [physicians in child and adolescent medicine 14,466, of which outpatient 7,244]: Structurally, these surgeries are best compared to the general practitioner's surgery, so that the results of the MFA survey could most likely be transferred to MFAs in such practices. Only the domiciliary visit function differs. The issue of prevention by vaccination has a much higher importance in paediatrician practices, since the basic immunizations are already being carried out in the infant age.

Specialists for geriatric: The (few) specialist surgeries with a focus on geriatrics show a similarly high transfer potential. In them, the domiciliary visit is rather in the foreground. In the case of vaccinations, the focus is clearly on influenza vaccination, as the older persons are among the risk groups, while the further vaccination protection has often been reached in the final. In a MFA survey, it is possible that the values for movement promotion and nutritional advice would be higher for this group, since the activity can be experienced for the MFA, where malnutrition and lack of exercise may lead to.

Internists [52,158, of which 25,289 outpatients]: Also, specialized practice with a focus on internal medicine has similar structures as general practitioners' surgeries. Even there, primary prevention (including vaccination) belongs to the original tasks, so that the related tasks for the MFA have a special meaning and influence their pref- 
erence profile with it. However, the "Internist" usually eliminates the obligation to make home visits. Since the other working environment is comparable to that of a general practitioner's surgery, it is likely that the list of priorities from the MFA survey would be very similar.

Diabetologists: For MFAs at diabetologists (Diabetes is one of the most widespread common diseases in Germany) is like MFAs in geriatrics. Their attitude towards personal health promotion measures is also likely to be influenced by the fact that they are given daily sight of the consequences of malnutrition and lack of exercise.

Gynaecologist and obstetrics [18,253, of which 11,500 outpatients]: The vaccination spectrum offered is essentially reduced to the HPV vaccination, which could also be carried out by the MFA. The tasks of the care of the physician are just as limited as the tasks of primary prevention, if one does not foresee that the advice of pregnant women is often directed towards movement, nutrition, alcohol and nicotine waiver.

Occupational physicians [3,282]: The range of vaccinations in the priority list is extended, as they often also make vaccination for travel. The use of the vaccinations recommended by the STIKO is currently not possible due to a gap in the Prevention Act (DGAUM, 2017). Therefore, the adoption of these tasks for MFAs is not yet a realistic perspective. Occupational physicians are often involved in the introduction of health management systems in large and midsize enterprises, thus further possibilities and concepts for health-promoting measures exist, in which MFAs are involved or could be involved. Occupational physicians are more often concerned with the stress-causing factors such as work organization, job design and leadership behaviour than general practitioners. The MFAs that support them are likely to have a higher sensitivity to these influencing factors of health.

In total, this will increase the number of MFAs that could benefit from the introduction of measures to promote personal health potential by a total of around 50,000 to a total of around 250,000 .

\subsubsection{MFAs in surgeries in other fields}

The surgeries mentioned above in which MFAs are active are characterized by the fact that they have a different, but still existing, proximity to primary prevention. This is not usually the case with other disciplines such as radiology, orthopaedics, urology, etc. Correspondingly, the medical competence is not exactly in the area of prevention and the MFA performs tasks in the medical field. (e.g. hearing tests at ear, nose and throat specialists).

For these disciplines, the results of the MFA survey and the priority list can only be applied with restriction, because some measures e.g. vaccinations or internal nutrition counselling cannot be executed. In addition, the medical specialists as competitors of general practitioners have a higher throughput and thus often also a more anonymous relation to the patient (cf. 2.2.3). This makes it difficult to live and propagate a whole-person approach, such as primary prevention. 
Although the priority list and the framework for the MFAs employed in these organizations (around 350,000 additional persons) is not directly implementable, it can be helpful in developing a "Healthy in Health Professions" mission statement.

\subsection{Limitations of the research}

The conclusions presented above were obtained from data from a survey of about 200 MFAs, the evaluation of studies and qualitative interviews with typed general practitioners. The design of data collection and evaluation was structured and documented in such a way that it leads to valid and replicable results. However, it must be recognised that, as with any research, there are limitations.

The MFA survey as the basis of the preference profile was created by surveying MFAs participating in training events. Limitations lie in the choice of participants, randomizing over the time and location as well as possible fashion trends in the healthcare sector.

The selection of participants was limited to those MFAs who could participate in training events. MFAs without readiness and/or opportunity to train may have different preferences.

Although the selection of events for the survey was based on a representative principle, it did not rule out possible distortions caused by random waves of disease (e.g. flu or measles waves affecting vaccination preferences). The same applies to the selection of the places where the surveys took place. In this way, it cannot be ruled out that regions with different social structures has also responded differently.

Limitations based on fashion trends in healthcare mean that different interest groups bring prevention-relevant health issues such as certain types of yoga, Xi gong, dietary supplements, diets, etc. to the fore in the media, which next season, according to a fashion trend, will no longer show up. This circumstance may have influenced the open question envisaged in the MFA survey.

Limitations in terms of studies concern, on the one hand, the selection of measures to promote personal health potential and, on the other hand, the structure of the operations model (Figure 46). The exclusive use of peer-reviewed articles is a harsh yardstick that may ultimately have led to prevention measures that are effective but not published in peer-reviewed articles were not included in the survey.

The limitations of the operations model lie in the modelling underlying assumptions, which were almost taken over during the further development. According to Katou (2013) these are the following four points:

First, there is a lack of psychological contract research referring to employer and employee promises fulfilment. Second, there is a lack of previous psychological contract research treating organizational performance as the ultimate 
dependent variable. Third, there is a scarcity of research considering that HR practices are antecedents of employer and employee promises fulfilment, especially for non-Western contexts investigating the HR practices - psychological contract - organizational performance relationship. Fourth, although the concept of psychological contract originated from outside HRM literature, it is used in explaining how HR practices predict employee attitudes and behaviours within the psychological contract.

Finally, it should be noted that a general limitation arises from the fact that it is ultimately an investigation and disclosure of personal health behaviour and lifestyle. Since this is more or less aware of all involved, for the MFAs as well as for the physicians, only the question of Majumdar (2004) concerning social desirability can be quoted: Do they want, what they should want?

\subsection{Future research}

This section subsumes the contributions from the previous sections and proposes further research that build on this DBA thesis. The main concern of this research is to further consolidate, stabilize and demonstrate a wider range of applications for the both models, the operational HRM-Model (Figure 46) and the decision-making model (Figure 47).

At the heart of the operational HRM-Model is the psychological contract, for which, according to the author, it should be investigated whether "mental models" (Biggs et al., 2011) can be used to achieve an even better understanding of the relationships, especially in the health sector. This applies both to the different concepts that the partners of the social contract associate with the respective content, such as employee satisfaction, as well as to the influencing factors, such as feedback for performance by the manager, which are culturally influenced and shaped by different experiences of socialization.

Further research could help to further investigate the relationship structure within the operations model and strengthen the empirical basis for the general practitioners' surgeries, including long-term studies. Addressing the largely unexplored leadership behaviour of physicians could help to better understand the dynamics of the model and gain a better insight into the relationship between leadership ability and organizational performance.

German General practitioners' surgeries are owner-managed micro-enterprises. For them, this DBA-research has developed an operational model that considers their specific HR practices, their specific interpretations of the psychological contract and their specific performance requirements. For similar structured micro-enterprises such as lawyers' and notaries' offices, the approach would have to be explored in terms of direct transferability. The transferability of the results is based on the target system for the standardization of health management in SMEs. For general practi- 
tioners' surgeries, it has proved to be an appropriate framework for the development of a priority approach. Further research could demonstrate the potential benefits, but also limitations, by standardizing health management in model development and implementation for SMEs that cannot afford comprehensive research themselves.

For SMEs that are not classified as micro-enterprises, the decision-making model comes into focus. As stated above, it can be used as a participatory model of procedure for the negotiation of the psychological contract. If one sees decision-making in complex situations as an important leadership responsibility and management task, further research into the scope of application of this approach could make a sustainable contribution to future leadership and management activities - both under theoretical and practical relevant aspects.

\subsection{Summary}

In summary, the author argues that this DBA-research study contributes to the body of knowledge both in developing and presenting models for operating and decisionmaking in health management of German practitioners' surgeries.

As a core contribution to empirical research in health management, the preference profile of MFAs and a priority concept for implementation are developed that takes employee preferences into account. The operational HRM model serves as a contribution to empirical research, which opens further starting points for debates on the link between HR practice and organisational performance, such as the fulfilment of the psychological contract in micro-enterprises like general practitioners' surgeries. The implementation of primary prevention based on the priority concept of measures of primary prevention is a further contribution to empirical research.

The core contribution to methodology is the decision-making model for general practitioners, on the basis of which the priority concept was developed taking into account different stakeholder perspectives. As a further contribution to the methodology, alternative generation is carried out in the decision-making model. A crossimpact analysis shows that the introduction of measures to promote personal health potential (primary prevention) compared to others like i.e. job design is most targeted.

The contribution to practice argues that no model for HRM and health management for German general practitioners' surgery is available and that the two models developed make a significant contribution to HRM and HR practices. Both models were focused on general practitioners in their development and were based on evaluations of the occupational field of MFAs. As a further contribution of practice, a check is carried out for other MFAs' fields of activities and profession (e.g. for specialist practices). 
The limitations result from the general difficulties that studies with a social science background raise. These relate to the evaluation of surveys, assumptions on model development and questions of social desirability.

Further research can explore relevance beyond micro-enterprises to SMEs in general, demonstrating the relevance of the two models in other environments as participative approaches. This would create additional benefits for HRM theories and HR practices in management and leadership. 


\section{References}

Preliminary note: The references contain numerous German-language articles. Not all authors and institutions have included in the biographical information a title translated into English. The English titles marked with $\left(^{*}\right)$ has been translated by the author of this research and not by the author(s) of the articles.

Abraham, C. and Graham-Rowe, E. (2009). Are worksite interventions effective in increasing physical activity? A systematic review and meta-analysis. Health Psychology Review 3: 108-144.

Amend, Christoph Thomas (2005) [Thesis]: Qualitätsmanagement in der Arztpraxis - Begleitung eines Managementprojekts im Ärztenetz MQLD ( ${ }^{*}$ Quality management in the doctor's office - Accompaniment of a management project in the medical network MQLD). Johann Wolfgang Goethe-Universität, Frankfurt am Main

Antonovsky (1997). Salutogenese. Zur Entmystifizierung der Gesundheit. ed. (* Salutogenesis. To demystify your health). Tübingen, dgvt-Verlag.

Appelbaum, E.; Bailey, T.; Berg, P. and Kalleberg, A.L. (2000). Manufacturing advantage: Why highperformance work systems pay off. ed. London, ILR Press.

Armstrong, M. (1996). A Handbook of Personnel Management Practice. 2nd ed. ed. London, Kogan Page.

Arthur, Jeffrey B. (1994). Effects of Human Resource Systems on Manufacturing Performance and Turnover. The Academy of Management Journal 37(3): 670-687.

Atkinson, C. (2005) [Thesis]: An Investigation into the Psychological Contract in Small Firms. Manchester Metropolitan University

Atkinson, C. and Cuthbert, P. (2006). Does one size fit all?: A study of the psychological contract in the UK working population. International Journal of Manpower 27(7): 647-665.

Atkinson, Carol (2007). Building high performance employment relationships in small firms. Employee Relations 29(5): 506-519.

Atkinson, Carol (2008). An exploration of small firm psychological contracts. Work, Employment \& Society 22(3): 447-465.

Atkinson, Carol and Hall, Laura (2011). Flexible working and happiness in the NHS. Employee Relations 33(2): 88-105.

Babitsch, B. and Von Moeller, K. (2017). Kompetenzanforderungen an MFA, ZFA, PKA und PTA - Konzipierung von wissenschaftlichen Weiterbildungsangeboten ( ${ }^{*}$ Competence requirements for MFA, ZFA, PCA and PTA - Design of scientific training courses), - 19. Hochschultage Berufliche Bildung an der Universität zu Köln. [Conference Paper]

Badura, B.; Greiner, W.; Rixgens, P.; Ueberle, M. and Behr, M. (2008).

Sozialkapital: Grundlagen von Gesundheit und Unternehmenserfolg. ed. (* Social captial: Fundamentals of health and organisations' success). Berlin, Heidelberg, Springer.

Baldry, C.; Taylor, P. and Bain, P. (2006). 'Bear with me': the problems of health and 
well-being in call centre work. in Wood, G. a. J., P. Institutions, Production and Working Life. Oxford, Oxford UP.

Bana e Costa, C. A.; De Corte, Jean-Marie and Vansnick, Jean-Claude (2014). MMACBETH. BANA Consulting. Retrieved from http://www.m-macbeth.com: 2016/09/26

Bana e Costa, C. and Vansnick, J.-C. (1999). The MACBETH approach: Basic ideas, software and an application. Advances in Decision Analysis 4: 131157.

Barbuto, J. and Scholl, R. (1998). Motivation sources inventory: Development and validation of new scales to measure an integrative taxonomy of motivation. Psychological Reports 82: 1011-1022.

Barney, Jay (1991). Firm Resources and Sustained Competitive Advantage. Journal of Management 17(1): 99-120.

Barr-Anderson, Daheia J (2011). Integration of Short Bouts of Physical Activity Into Organizational Routine: A Systematic Review of the Literature. American journal of preventtive medicine 40(1): 76-93.

Barré, de la (2014). Gesundes Arbeiten in der Arztpraxis - Betriebliches Gesundheitsmanagement schafft Mehrwert für Praxisinhaber und Mitarbeiter. ( ${ }^{*}$ Healthy work in the physician's office - Occupational health management creates added value for practice owners and employees). Frauenarzt 55: 1267 - 1269.

Bartram, T. (2005). Small firms, big ideas: The adoption of human resource management in Australian small firms. Asia Pacific Journal of Human Resources 43(1): 137-154.

Baxter, David (2007). Specific immunization issues in the occupational health setting. Occupational Medicine 57(8): 557-563.

Becker, Brian and Gerhart, Barry (1996). The Impact of Human Resource Management on Organizational Performance: Progress and Prospects. The Academy of Management Journal 39(4): 779-801.

Becker, Thomas E. (1992). Foci and Bases of Commitment: Are They Distinctions worth Making? The Academy of Management Journal 35(1): 232-244.

Benedict, MA and Arterburn, D (2008). Worksite-based weight loss programs: a systematic review of recent literature. American Journal of Health Promotion 22(6): 408-416.

Berger, Lisa K.; Sedivy, Sonya K.; Cisler, Ron A. and Dilley, Lorna J. (2008). Does job satisfaction mediate the relationships between work environment stressors and employee problem drinking? Journal of Workplace Behavioral Health 23(3): 229-243.

Berufsbildung, Forum (2017). [Web Page] Forum Berufsbildung. Retrieved from https://www.forum-berufsbildung.de/beratungservice/berufsbilder/medizinischer-fachangestellter-medizinischefachangestellte/: 2017/09/29

BGW (2017). Gefährdungsbeurteilung in der Arztpraxis. ( ${ }^{*}$ Risk assessment in the physician's office). Berufsgenossenschaft für Gesundheit und Wohlfahrtspflege (BGW). [Electronic Article]

BIBB (2011). Rangliste 2011 der Ausbildungsberufe nach Neuabschlüssen in Deutschland. ( ${ }^{*}$ Ranking 2011 of the training occupations after new contracts in Germany.). [Electronic Article] 
BIBB (2016). Rangliste 2016 der Ausbildungsberufe nach Neuabschlüssen in Deutschland. (Ranking 2016 of the training occupations after new contracts in Germany.). [Electronic Article]

Biggs, Duan; Abel, Nick; Knight, Andrew T.; Leitch, Anne; Langston, Art and Ban, Natalie C. (2011). The implementation crisis in conservation planning: could "mental models" help? Conservation Letters 4(3): 169-183.

Bilban, Marjan (2005). Occupational medicine in the Slovene area. Journal Of Occupational Health 47(3): 193-200.

Blumberg, Melvin and Pringle, Charles D. (1982). The Missing Opportunity in Organizational Research: Some Implications for a Theory of Work Performance. The Academy of Management Review 7(4): 560-569.

BMG (2016). Daten des Gesundheitswesen 2016. ( ${ }^{*}$ Healthcare data 2016). Bundesministerium für Gesundheit

Boselie, J. P. P. E. F.; Dietz, G. and Boon, C. (2005). Commonalities and contradictions in research on human resource management and performance. Human Resource Management Journal 15(3): 67-94.

Bowen, David E. and Ostroff, Cheri (2004). Understanding HRM-Firm Performance Linkages: The Role of the "Strength" of the HRM System. The Academy of Management Review 29(2): 203-221.

Boxall, P. and Macky, K. (2007). High-performance work systems and organisational performance: Bridging theory and practice-, SAGE PUBLICATIONS INC. [Manuscript]

Boxall, P.; Purcell, J. and Wright, P. M. (2008). Human Resource Management : Scope, Analysis, and Significance. The Oxford Handbook of Human Resource Management, Oxford University Press.

Boxall, Peter F. and Purcell, John (2003). Strategy and human resource management. ed. Basingstoke, Hampshire, Palgrave Macmillan.

Braun, Philipp (2016) [Thesis]: Gesundheitsmanagement in Deutschland Perspektiven deutscher Mitarbeiter und Reaktionsmöglichkeiten moderner Organisationen. ( ${ }^{*}$ Health Management in Germany - perspectives of German employees and the reaction possibilities of modern organizations). Universität Köln

Bräunig, D.; Haupt, J.; Kohstall, T.; Kramer, I.; Pieper, C. and Schröer, S. (2015). iga.Report 28 - Wirksamkeit und Nutzen betrieblicher Prävention. (* iga.Report 28 - Efficacy and benefits of occupational prevention). Deutsche Gesetzliche Unfallversicherung (DGUV)

Brewer, S.; Van Eerd, D.; Amick, B. C., III; Irvin, E.; Daum, K. M.; Gerr, F.; . . . Rempel, D. (2006). Workplace interventions to prevent musculoskeletal and visual symptoms and disorders among computer users: a systematic review. Journal of Occupational Rehabilitation 16(3): 325-358.

Brooke, Paul P. (1986). Beyond the Steers and Rhodes Model of Employee Attendance. The Academy of Management Review 11(2): 345.

Bruch, H. and Kowalevski, S. (2013). Gesunde Führung. Wie Unternehmen eine gesunde Performancekultur entwickeln. ( ${ }^{*}$ Healthy leadership. How companies develop a healthy performance culture). St. Gallen: Compamedia $\mathrm{GmbH}$. [Electronic Article]

Brussig, Martin and Ahlers, Elke (2007). Krankheitsbedingte Fehlzeiten älterer Arbeitnehmer und Arbeitnehmerinnen im betrieblichen Kontext. (Sickness- 
related periods of absence from work of elderly workers from a company context). Industrielle Beziehungen: Zeitschrift für Arbeit, Organisation und Management 14(4): 357-378.

Bundesärztekammer (2017). Ärztestatistik 2016: Die Schere zwischen Behandlungsbedarf und Behandlungskapazitäten öffnet sich. ( ${ }^{*}$ Medical statistics 2016: The gap between treatment needs and treatment capacities opens up).

Bundesanzeiger (2006). Verordnung über die Berufsausbildung zum Medizinischen Fachangestellten/zur Medizinischen Fachangestellten. Verordnung über die Berufsausbildung zum Medizinischen Fachangestellten/zur Medizinischen Fachangestellten.

Bundesinstitut für Berufsbildung BIBB (2012). Gelernte Medizinische Fachangestellte im Beruf - Ergebnisse der BIBB/BAuAErwerbstätigenbefragung 2012 --. [Aggregated Database]

Bunke, J. F. (2008). Elimination of manual patient handling to prevent work-related musculoskeletal disorders. Bariatric Nursing \& Surgical Patient Care 3(4): 262-262.

Burrell, Gibson and Morgan, Gareth (1982). Sociological Paradigms and Organisational Analysis: Elements of the Sociology of Corporate Life. ed., Routledge.

Byrns, G. E.; Palatianos, K. H.; Shands, L. A.; Fennelley, K. P.; McCammon, C. S.; Boudreau, A. Y.; . . . Mitchell, C. S. (2000). Chemical hazards in radiology. Applied Occupational And Environmental Hygiene 15(2): 203-208.

Caban-Martinez, Alberto J.; Lee, David J.; Davila, Evelyn P.; LeBlanc, William G.; Arheart, Kristopher L.; McCollister, Kathryn E.; . . . Fleming, Lora E. (2010). Sustained low influenza vaccination rates in US healthcare workers. Preventive Medicine: An International Journal Devoted to Practice and Theory 50(4): 210-212.

Cahill, K.; Hartmann-Boyce, J. and Perera, R. (2015). Incentives for smoking cessation. Nuffield Department of Primary Care Health Sciences, University of Oxford

CIPD (2008). The psychological contract-. London, Chartered Institute of Personnel and Development. [Magazine Article]

CIPD (2016). Growing the Health and Well-Being Agenda: From First Steps to Full Potential-. London, Chartered Institute of Personnel and Development. [Magazine Article]

Cloud, John (2009). Staying Sane May Be Easier Than You Think.- Time, Time Inc. 173: 72-78. [Magazine Article]

Commission, European Union (2003). Kleinstunternehmen sowie kleine und mittlere Unternehmen: Definition und Umfang EU L 124/36. (Micro-, small and medium-sized enterprises: definition and scope EU L 124/36), - [Government Document]

Coyle-Shapiro, Jackie and Kessler, Ian (2000). Consequences Of The Psychological Contract For The Employment Relationship: A Large Scale Survey*. Journal of Management Studies 37(7): 903-930.

Coyle-Shapiro, Jacqueline A. M. (2002). A psychological contract perspective on organizational citizenship behavior. Journal of organizational behavior. 23(8): 927. 
Coyle-Shapiro, Jacqueline A. M. and Kessler, Ian (2003). The employment relationship in the U.K. public sector: A psychological contract perspective. Journal of Public Administration Research and Theory 13(2): 213.

Delaney, John T. and Huselid, Mark A. (1996). The Impact of Human Resource Management Practices on Perceptions of Organizational Performance. The Academy of Management Journal 39(4): 949-969.

Delery, John E. and Doty, D. Harold (1996). Modes of theorizing in strategic human resource management: Tests of universalistic, contingency, and configurational performance predictions. Academy of Management Journal 39(4): 802.

DGAUM (2017). Versorgung mit Schutzimpfungen durch Betriebsärzte: Vorschläge von DGAUM und BARMER zu einem bundesweit geltenden Vertragswerk ( Supply of vaccinations by company doctors: suggestions by DGAUM and BARMER on a nationwide agreement), -, DGAUM Deutsche Gesellschaft für Arbeitsmedizin und Umweltmedizin e. V. [Standard]

Dini, L.; Heintze, C.; Braun, V. and Sarganas, G. (2012). Delegierung von Hausbesuchstätigkeiten an Medizinische Fachangestellte: Akzeptanz unter den Hausärzten Mecklenburg-Vorpommerns. (Home Visit Delegation in Primary Care). Deutsches Arzteblatt International 109(46): 795-801.

Dini, L.; Hirth, M. and Heintze, C. (2018). Delegation in der Hausarztpraxis Ergebnisse einer Befragung von Hausärztinnen und Hausärzten in Nordrhein-Westfalen. Abschlussbericht. (Delegation to the family doctor's office - Results of a survey of family doctors in North Rhine-Westphalia. Final report).

Djeriri, K.; Laurichesse, H.; Merle, J. L.; Charof, R.; Abouyoub, A.; Fontana, L.; . . Beytout, J. (2008). Hepatitis B in Moroccan health care workers. Occupational Medicine 58(6): 419-424.

Dorfman, Sharon L. (2000). Preventive Interventions under Managed Care: Mental Health and Substance Abuse Services. ed., University of Michigan Library.

Doumas, Diana M. and Hannah, Elizabeth (2008). Preventing high-risk drinking in youth in the workplace: A web-based normative feedback program. Journal of Substance Abuse Treatment 34(3): 263-271.

Dowdle, Walter; Van der Avoort, Harrie; De Gourville, Esther; Delpeyroux, Francis; Desphande, Jagadish; Hovi, Tapani; . . . Wolff, Chris (2006). Containment of Polioviruses After Eradication and OPV Cessation: Characterizing Risks to Improve Management. Risk Analysis: An International Journal 26(6): 14491469.

Dugdill, L.; Brettle, A.; Hulme, C.; McCluskey, S. and Long, AF. (2008). Workplace Physical Activity Interventions: a Systematic Review. International Journal of Workplace Health Management 1(1): 20-40.

Dunn, Robert (2008). Immunisation policy. Occupational Health 60(12): 39-41.

Ehrnrooth, Mats and Björkman, Ingmar (2012). An Integrative HRM Process Theorization: Beyond Signalling Effects and Mutual Gains. The Journal of Management Studies 49(6): 1109.

Eikamp, Jörg (2015) [Thesis]: Zur Gesundheit von klinisch tätigen Ärztinnen und Ärzten in Deutschland - Ergebnisse mit Hilfe eines Online-Instrumentes zum Betrieblichen Gesundheitsmanagement. ( ${ }^{*}$ On the health of clinically active physicians in Germany - results with the help of an online instrument for occupational health management). Universität Bielefeld 
Emmett, Edward A. (2002). Occupational contact dermatitis I: incidence and return to work pressures. American Journal Of Contact Dermatitis: Official Journal Of The American Contact Dermatitis Society 13(1): 30-34.

Engbers, Luuk (2007). Monitoring and Evaluation of Worksite Health Promotion Programs - Current state of knowledge and implications for practice World health Organisation

Esterhuizen, T. M.; Hnizdo, E. and Rees, D. (2001). Occurrence and causes of occupational asthma in South Africa--results from SORDSA's Occupational Asthma Registry, 1997-1999. South African Medical Journal = SuidAfrikaanse Tydskrif Vir Geneeskunde 91(6): 509-513.

Esterhuizen, T. M.; Hnizdo, E.; Rees, D.; Lalloo, U. G.; Kielkowski, D.; van Schalkwyk, E. M.; . . Curtis, T. (2001). Occupational respiratory diseases in South Africa--results from SORDSA, 1997-1999. South African Medical Journal = Suid-Afrikaanse Tydskrif Vir Geneeskunde 91(6): 502-508.

Falk, Stephanie (2010). Der harte Alltag in einer Hausarztpraxis - Diskrepanzen in der ambulanten vertragsärztlichen Versorgung. ( ${ }^{*}$ The harsh everyday life in a family practice - discrepancies in the outpatient contract medical care), -. http://www.arztpraxis-roloff.de/2010/Hausarbeit-St-Falk.pdf. [Manuscript] Student research project

Fallentin, Nils (2003). Regulatory actions to prevent work-related musculoskeletal disorders--the use of research-based exposure limits. Scandinavian Journal of Work, Environment \& Health 29(4): 247-250.

Faucett, Julia; Garry, Mary; Nadler, Don and Ettare, Dennis (2002). A test of two training interventions to prevent work-related musculoskeletal disorders of the upper extremity. Applied Ergonomics 33(4): 337-347.

Fazliogullari, O. (2012). Scientific Research Paradigms in Social Sciences. International Journal of Educational Policies 6(1): 41-55.

Frodl, Andreas (2016). Praxisführung für Ärzte - Kosten senken, Effizienz steigern. ( ${ }^{*}$ Practical guidance for doctors - reduce costs, increase efficiency). Springer Fachmedien Wiesbaden. [Electronic Book]

Bundesanzeiger (2016). Qualitätsmanagement-Richtlinie/QM-RL. ( ${ }^{*}$ Quality management policy / QM RL). Qualitätsmanagement-Richtlinie/QM-RL. (BAnz AT 15.11.2016 B2)

Gavartina, Amina; Zaroti, Stavria; Szecsenyi, Joachim; Miksch, Antje; Ose, Dominik; Campbell, Stephen M and Goetz, Katja (2013). Practice assistants in primary care in Germany - associations with organizational attributes on job satisfaction. BMC Family Practice 14.

Gehring, Katrin and Schwappach, David (2014). Patientensicherheit in der Hausarztpraxis. (Patient safety in general practice). Zeitschrift für Evidenz, Fortbildung und Qualität im Gesundheitswesen (ZEFQ) 108: 25-31.

Gerlach, Ferdinand M. (2001). Qualitätsförderung in Praxis und Klinik : eine Chance für die Medizin. ed. Stuttgart, Thieme.

Gibbs, Vivien and Young, Pat (2011). A study of the experiences of participants following attendance at a workshop on methods to prevent or reduce workrelated musculoskeletal disorders amongst sonographers. Radiography 17(3): 223-229.

GKV-Spitzenverband (2014). Leitfaden Prävention Handlungsfelder und Kriterien des GKV-Spitzenverbandes zur Umsetzung der §§ 20 und 20a SGB V vom 
21. Juni 2000 in der Fassung vom 10. Dezember 2014. ( ${ }^{*}$ Guidelines for prevention Fields of action and criteria of the GKV-Spitzenverbandes for the implementation of $\S \S 20$ and 20a SGB V of 21 June 2000 in the version of 10 December 2014).

Goetz, Katja; Berger, Sarah; Gavartina, Amina; Zaroti, Stavria and Szecsenyi, Joachim (2015). How psychosocial factors affect well-being of practice assistants at work in general medical care? - a questionnaire survey. BMC Family Practice 16.

Goetz, Katja; Campbell, Stephen M; Steinhaeuser, Jost; Broge, Bjoern; Willms, Sara and Szecsenyi, Joachim (2011). Evaluation of job satisfaction of practice staff and general practitioners: an exploratory study. BMC Family Practice 12.

Gomez-Mejia, Luis R. (2004). Managing human resources. 3rd Canadian ed. ed. Toronto, Prentice Hall.

Gomez, P. and Probst, G. (1997). Die Praxis des ganzheitlichen Problemlösens Vernetzt denken - Unternehmerisch handeln - Persönlich überzeugen. ed. ( ${ }^{*}$ The practice of holistic problem solving - network thinking - acting entrepreneurially - convincing personally). Haupt, Bern.

Graham, Annette L.; Julian, John and Meadows, Graham (2010). Improving responses to depression and related disorders: Evaluation of a innovative, general, mental health care workers training program. International Journal of Mental Health Systems 4(25).

Greasley, Kay; Edwards, Paul; Baker-McClearn, Denise and Dale, Jeremy (2012). Why do organisations engage in HR initiatives? A test case of a health and wellbeing intervention. Employee Relations 34(4): 443-462.

Grimm, Pamela (2010). Social Desirability Bias. Wiley International Encyclopedia of Marketing, John Wiley \& Sons, Ltd.

Grobe, T. G. ; Dörning, M.; Mieth, I.; Held, R.; Reuther, U. and Schoppmeyer, U. (2011). Gesundheitsreport 2011 Bayern. ( ${ }^{*}$ Health report Bavaria 2011). ISEG Institut für Sozialmedizin, Epidemiologie und Gesundheitssystemforschung - ZFBT Zentrum Frau in Beruf und Technik

Grobe, Thomas; Steinmann, Susanne and AQUA, - Institut für angewandte Qualitätsforschung und Forschung im Gesundheitswesen $\mathrm{GmbH}$ (2016). Gesundheitsreport 2016 Gesundheit zwischen Beruf und Familie. (Health Report 2016 - Health between work and family). Techniker Krankenkasse (TK)

Guest, D. (1998). A winning combination: Mark Huselid's ideas and evidence for a solid link between 'bundles' of HR practices and business gain. PEOPLE MANAGEMENT: 64-67.

Hackman, J. R. and Oldham, G. R. (1976). Motivation through the design of work: Test of a theory. Organizational Behavior and Human Performance 16: 250279.

Hall, L. (2013). Flu -- to jab or not to jab? Occupational Health 65(11): 15.

Handschuch, Mandy; Schreiner-Kürten, Karin and Wanek, Volker (2015). Leitfaden Prävention - Handlungsfelder und Kriterien des GKV-Spitzenverbandes zur Umsetzung der $\S \S 20$ und 20a SGB V vom 21. Juni 2000 in der Fassung vom 10. Dezembe 2014. ( ${ }^{*}$ Guideline Prevention - Fields of Action and Criteria of the GKV-Spitzenverband for the Implementation of Sections 20 and 20a SGB V of 21 June 2000 in the version of 10 December 2014), - . 
GKV-Spitzenverband: 110. [Government Document]

Hargreaves, Carol (2008). A safe pair of hands. Occupational Health 60(11): 39-41.

Haukka, E.; Leino-Arjas, P.; Viikari-Juntura, E.; Takala, E.; Malmivaara, A.; Hopsu, L.; . . Riihimaki, H. (2008). A randomised controlled trial on whether a participatory ergonomics intervention could prevent musculoskeletal disorders. Occupational \& Environmental Medicine 65(12): 849-856.

Hedges, J. N. (1973). Absence from work - A look at some national data. Monthly Labor Review 96: 24-31.

Helmenstein, C.; Kleissner, M. H. A.; Riedel, M.; Röhrling, G. and Schnabl, A. (2004). Endbericht: Ökonomischer Nutzen betrieblicher Gesundheitsförderung - Studie im Auftrag des Bundeskanzleramts. ( ${ }^{*}$ Final Report: Economic Benefits of Workplace Health Promotion - Study commissioned by the Federal Chancellery (Austria)). Institut für Höhere Studien (IHS), Wien und ESCE Wirtschafts- und Sozialwissenschaftliche Forschung, Eisenstadt

Henry, Gary T. (1990). Practical Sampling. ed. Newbury Park, Sage.

Hill, J. M. and Trist, E. L. (1955). Changes in accidents and other absences with length of service. Human Relations 8: 121-152.

Hnizdo, E.; Esterhuizen, T. M.; Rees, D. and Lalloo, U. G. (2001). Occupational asthma as identified by the Surveillance of Work-related and Occupational Respiratory Diseases programme in South Africa. Clinical And Experimental Allergy: Journal Of The British Society For Allergy And Clinical Immunology 31(1): 32-39.

Höhl, Rebekka (2013). Geizen Praxichefs beim Gehalt? - Medizinische Fachangestellte. (Stingy practice heads in salary? - Medical assistant). UroNews 17: 60-60.

Holmqvist, Marika; Hermansson, Ulric and Nilsen, Per (2008). TOWARDS INCREASED ALCOHOL INTERVENTION ACTIVITY IN SWEDISH OCCUPATIONAL HEALTH SERVICES. International Journal of Occupational Medicine \& Environmental Health (Instytut Medycyny Pracy im. Jerzego Nofera) 21(2): 179-187.

Huselid, Mark A. (1995). The Impact of Human Resource Management Practices on Turnover, Productivity, and Corporate Financial Performance. The Academy of Management Journal 38(3): 635-672.

Imai, Masaaki (2002). Kaizen - der Schlüssel zum Erfolg im Wettbewerb. 2. Aufl. ed. ( ${ }^{*}$ Kaizen - the key to success in the competition). München, Econ.

Imarinen, Juhani and Tempel, Jürgen (2002). Arbeitsfähigkeit 2010 - Was können wir tun, damit Sie gesund bleiben? ( ${ }^{*}$ Working ability 2010 - What can we do to help you stay healthy?). [Electronic Book]

infas (2016). Tabellenband - Ärztemonitor 2016: Ergebnisse für Haus- und Fachärzte. ( ${ }^{*}$ Table band - Doctors Monitor 2016: results for GPs and specialists). Kassenärztlichen Bundesvereinigung (KBV) und Verband der niedergelassenen Ärzte Deutschlands e.V., (NAV-Virchow-Bund)

Institut für betriebswirtschaftliche Analysen, Beratung und Strategie-Entwicklung (IFABS) (2012). Untersuchung der Arbeitszufriedenheit von MFA. ( ${ }^{*}$ Study of work satisfaction of MFA). PPA Praxisteam profession (Arztpraxis) 07/2012.

Ishizaka, Alessio and Nemery, Philippe (2013). Multi-criteria decision analysis: methods and software. ed. Cichester, John Wiley \& Sons, Ltd. 
Jacob, Rüdiger (2009). Methoden und Techniken der empirischen Sozialforschung. (Methods and Techniques of Empirical Social Research), -. [Manuscript]

Juran, J.M. (1989). Juran on Leadership for Quality: An Executive Handbook-, Free Press, New York. [Classical Work]

Kaplan, Robert S. and Norton, David P. (1996). The Balanced Scorecard: Translating Strategy Into Action ed., Mcgraw-Hill Professional.

Kathmann, Till and Dingeldey, Irene (2013). Prekarisierung berufsfachlich qualifizierter Beschäftigung? - Eine Analyse der Arbeitsbedingungen von medizinischen Fachangestellten. ( ${ }^{*}$ Precarization of vocationally qualified employment? - An analysis of the working conditions of medical assistants). Institut Arbeit und Wirtschaft (IAW) Universität/Arbeitnehmerkammer Bremen

Katou, Anastasia (2013). The link between HR practices, psychological contract fulfilment, and organisational performance in Greece: an economic crisis perspective. School of Industrial and Aeronautic Engineering of Terrassa (ETSEIAT). Universitat Politècnica de Catalunya (UPC) 2013-07. [Electronic Article]

KBV (2015). Qualitätsmanagement in der Praxis. (Quality management in surgeries). Kassenärztliche Bundesvereinigung (KBV). [Electronic Article]

Kelemen, Mihaela $L$ and Rumens, Nick (2008). An Introduction to Critical Management Research. ed. London, SAGE Publications Ltd.

Kinnie, N.; Hutchinson, S.; Purcell, J.; Rayton, B. and Swart, J. (2005). Satisfaction with HR practices and commitment to the organisation: why one size does not fit all. Human resource management journal. 15(4): 9-29.

KMK (2005). RAHMENLEHRPLAN für den Ausbildungsberuf Medizinischer Fachangestellter/Medizinische Fachangestellte. ( ${ }^{*}$ Framework curriculum for the vocatinal training of Medical assistant (MFA)), -. [Government Document]

KMK (2006). Verordnung über die Berufsausbildung zum Medizinischen Fachangestellten/zur Medizinischen Fachangestellten. ( ${ }^{*}$ Regulation on vocational training for medical assistants / for medical assistants), -. [Government Document]

Knight, Andrew T.; Cowling, Richard M. and Campbell, Bruce M. (2006). An Operational Model for Implementing Conservation Action. Conservation Biology 20(2): 408-419.

Köper, Birgit; Möller, Klaus and Zwetsloot, Gerard (2009). The occupational safety and health scorecard -- a business case example for strategic management. Scandinavian Journal of Work, Environment \& Health 35(6): 413-420.

KVB (2017). Neue Qualitätsmanagement-Richtlinie (QM-RL). ( ${ }^{*}$ New Quality Management Policy), - Qualität. [Magazine Article]

Laaksonen, M.; Piha, K.; Martikainen, P.; Rahkonen, O. and Lahelma, E. (2009). Health-related behaviours and sickness absence from work. Occupational \& Environmental Medicine 66(12): 840-847.

Larsson, Johan; Landstad, Bodil and Vinberg, Stig (2009). To control with health: From statistics to strategy. Work 32(1): 49.

Lemon, S. C. and Pratt, C. A. (2010). Worksite environmental interventions for obesity control: an overview. Journal of Occupational \& Environmental Medicine 52(1S): S1-3.

Likert, R. (1967). The human organization: Its management and value-. New York, 
McGraw-Hill. [Classical Work]

Little, K. E.; Goodrigde, S.; Lewis, H.; Lingard, S. W.; Din, S.; Tidley, M. and Hayes, S. (2015). Occupational vaccination of health care workers: Uptake, attitudes and potential solutions. Public Health 129(6): 755-762.

Lüerßen, Hartmut; Stickling, Erwin; Gundermann, Nils; Toska, Marko; Coppik, Robert; Denker, Philipp; . . Timmerhoff, Christian (2015). BGM im Mittelstand 2015 - Ziele, Instrumente und Erfolgsfaktoren für das Betriebliche Gesundheitsmanagement. ( ${ }^{*}$ HM in SMEs 2015 - Goals, Instruments and Success Factors for Occupational Health Management).

Maar, Christa; Fricker, Rolf; Hildebrandt, Nicole and Drechsler, Melanie (2011). Vorteil Vorsorge: Die Rolle der betrieblichen Gesundheitsvorsorge für die Zukunftsfähigkeit des Wirtschaftsstandortes Deutschland. ( ${ }^{*}$ Benefits: The role of occupational health care for the future viability of Germany as a business location). Felix Burda Stiftung / Booz \& Company

MacInnis, Deborah J. and Jaworski, Bernard J. (1989). Information Processing from Advertisements: Toward an Integrative Framework. Journal of Marketing 53(4): 1-23.

Madsen, Ida E. H.; Burr, Hermann; Diderichsen, Finn; Pejtersen, Jan H.; Borritz, Marianne; Bjorner, Jakob B. and Rugulies, Reiner (2011). Work-related Violence and Incident Use of Psychotropics. American Journal of Epidemiology 174(12): 1354-1362.

Mandl, Heinz and Gerstenmaier, Jochen (2000). Die Kluft zwischen Wissen und Handeln - Empirische und theoretische Lösungsansätze. ed. ( ${ }^{*}$ The gap between knowledge and action - Empirical and theoretical solutions). Göttingen, Hogrefe Verlag für Psychologie.

Marcks, Sarah Alexandra Stefanie (2012) [Thesis]: Auswirkungen von Qualitätsmanagement in der Hausarztpraxis (Allgemeinarztpraxis und hausärztlich internistische Arztpraxis) - Ergebnisse einer strukturierten Befragung der Interessengruppen. (Effects of Quality Management in Family Practice - Results of a Structured Survey of Stakeholders).

Marin-Garcia, Juan A. and Tomas, Juan Martinez (2016). Deconstructing AMO framework: a systematic review. [Electronic Article]

Marlow, Susan (2002). Regulating labour management in small firms. Human Resource Management Journal 12(3): 25-43.

Marsden, D. and Moriconi, S. (2009). 'The value of rude health: Employees' well being, absence and workplace performance- CEP Discussion Paper No. 919. London, Centre for Economic Performance. [Generic]

Matson-Koffman, DM; Brownstein, JN; Neiner, JA and Greaney, ML (2005). A sitespecific literature review of policy and environmental interventions that promote physical activity and nutrition for cardiovascular health: what works? Health Promotion.

Mattare, M.; Shah, A. and Monahan, M. (2013). HUMAN RESOURCE MANAGEMENT IN MICROENTERPRISES: GATEWAY FOR SUCCESS. The Costal Business Journal 12 (1): 20-31.

Mayo, Elton (1927-1932). Hawthorne and the Western Electric Company-. [Classical Work]

McDonald, David J. and Makin, Peter J. (2000). The psychological contract, organisational commitment and job satisfaction of temporary staff. 
Leadership \& Organization Development Journal 21(2): 84-91.

Meijster, Tim; van Duuren-Stuurman, Birgit; Heederik, Dick; Houba, Remko; Koningsveld, Ernst; Warren, Nicholas and Tielemans, Erik (2011). Costbenefit analysis in occupational health: a comparison of intervention scenarios for occupational asthma and rhinitis among bakery workers. Occupational And Environmental Medicine 68(10): 739-745.

Melhorn, J. M. and Gardner, P. (2004). How we prevent prevention of musculoskeletal disorders in the workplace. Clinical Orthopaedics \& Related Research 419: 285-296.

Mergenthal, K.; Beyer, M.; Güthlin, C. and Gerlach, F. M. (2013). Evaluation des VERAH-Einsatzes in der Hausarztzentrierten Versorgung in BadenWürttemberg. (Evaluating the deployment of VERAHs in family doctorcentred health care in Baden-Wuerttemberg). Zeitschrift fur Evidenz, Fortbildung und Qualitat im Gesundheitswesen 107(6): 386-393.

Messersmith, Jake G.; Patel, Pankaj C. and Lepak, David P. (2012). "Unlocking the black box: Exploring the link between high-performance work systems and performance": Correction to Messersmith et al. (2011). Journal of Applied Psychology 97(4): 824.

Millward, L. J. and Brewerton, P. M. (1999). Contractors and their psychological contracts. British Journal of Management 10(3): 253-274.

Moeller, K. von and Babitsch, B. (2017). Prävention und Gesundheitsförderung Ein Thema auch für die wissenschaftliche Weiterbildung. ( ${ }^{*}$ Prevention and Health Promotion - A topic also for the scientific further education). Das Gesundheitswesen 79(8/09): 619-626.

Morrow, Lou; Verins, Irene and Willis, Eileen (2002). Mental Health and Work: Issues and Perspectives. Australian Network for Promotion, Prevention and Early Intervention.

Müller-Armack (1976). Wirtschaftsordnung und Wirtschaftspolitik. ed. ( ${ }^{*}$ Economic order and economic policy). Haupt, Bern.

Muzi, G.; Murgia, N.; Abbritti, G.; Tinozzi, C. and dell'Omo, M. (2006). [Respiratory diseases in confined non-industrial working environments]. Giornale Italiano Di Medicina Del Lavoro Ed Ergonomia 28(3): 276-278.

Mytton, O. T.; O'Moore, E. M.; Sparkes, T.; Baxi, R. and Abid, M. (2013). Knowledge, attitudes and beliefs of health care workers towards influenza vaccination. Occupational Medicine 63(3): 189-195.

N., N. (2001). Clinical update in musculoskeletal medicine. "Joint motion" gaining momentum: worldwide effort to research, manage, and prevent musculoskeletal disorders. Journal of Musculoskeletal Medicine 18(1): 41-42.

National Association for Mental Health, Incorporated (1929). The American Foundation for Mental Hygiene: its origins, purposes, and personnel. Mental Hygiene 4: 673-677.

Nishii, Lisa H.; Lepak, David P. and Schneider, Benjamin (2008). EMPLOYEE ATTRIBUTIONS OF THE "WHY" OF HR PRACTICES: THEIR EFFECTS ON EMPLOYEE ATTITUDES AND BEHAVIORS, AND CUSTOMER SATISFACTION. Personnel Psychology 61(3): 503-545.

Oreg, Shaul; Michel, Alexandra and By, Rune Todnem (2013). The psychology of organizational change : viewing change from the employee's perspectiveCambridge, Cambridge University Press. [Electronic Book] 
Oswald, Margit E. and Grosjean, Stefan (2004). Confirmation Bias. Cognitive Illusions: A Handbook on Fallacies and Biases in Thinking, Judgement and Memory. Hove, UK, Psychology Press: 79-96.

Paauwe, Jaap and Richardson, Ray (1997). Introduction. The International Journal of Human Resource Management 8(3): 257-262.

Pate, Judy; Martin, Graeme and McGoldrick, Jim (2003). The impact of psychological contract violation on employee attitudes and behaviour. Employee Relations 25(6): 557-573.

Patterson, Malcolm; West, Michael; Lawthom, Rebecca and Nickell, S. (1997). Impact of people management practices on business performance-, Institute of Personnel and Development. [Manuscript]

Patton, M.Q. (2002). Qualitative research and evaluation methods. 3rd ed. ed., Thousand Oak: Sage.

Peccei, R.; Van de Voorde, K. and Van Veldhoven, M. (2013). HRM, well-being and performance: A theoretical and empirical review. in Paauwe, J., Guest, D. and Wright, P. HRM and performance: Achievements and Challenges. Cichester, Wiley.

Peccei, Riccardo (2004). Human Resource Management And The Search For The Happy Workplace. ed.

Pernerstorfer, Georg (2009). Konfidenzintervall für den Parameter $p$ einer Binomialverteilung ( ${ }^{*}$ Confidence interval for the parameter $p$ of a binomial distribution). mathe online. [Electronic Article]

Piening, Erk P.; Baluch, Alina M. and Ridder, Hans-Gerd (2014). Mind the IntendedImplemented Gap: Understanding Employees' Perceptions of HRM. Human Resource Management 53(4): 545-567.

Porter, M. (1980). Competitive Strategy. ed. New York, Free Press.

Preece, Richard (2012). Improving influenza immunisation uptake in healthcare workers. Occupational Health 64(4): 12-13.

Price, James L. (1972). Handbook of organizational measurement. ed. Lexington, Mass., Heath.

Price, James L. (1977). The study of turnover. 1st ed. ed. Ames, lowa State University Press.

Price, James L. and Mueller, Charles W. (1981). Professional turnover : the case of nurses. ed. New York, SP Medical \& Scientific Books.

Price, James L. and Mueller, Charles W. (1985). Handbook of organizational measurement. ed. Boston, Pitman.

Regus (2012) [Thesis]: Die Rolle des Hausarztes in der Prävention am Beispiel der Gesundheitsuntersuchung und präventiven Beratung. (The role of the general practitioner in prevention with the example of health examination and preventive counselling). Universitätsmedizin Berlin

Rhodes, Susan R and Steers, Richard M (1978). Major influences on employee attendance: A process model. Journal of applied Psychology 63(4): 391.

Rivilis, I; van Eerd, D; Cullen, K; Cole, DC; Irvin, E; Tyson, J and Mahood, Q (2008). Effectiveness of participatory ergonomic interventions on health outcomes: a systematic review. Applied Ergonomics: 342-358.

RKI (2017). [Web Page] Saisonale Influenzaimpfung: Häufig gestellte Fragen und 
Antworten. (Seasonal influenza vaccination: Frequently Asked Questions and Answers) Retrieved from

https://www.rki.de/SharedDocs/FAQ/Impfen/Influenza/faq_ges.html: 201710-04

Robinson, Sandra L.; Kraatz, Matthew S. and Rousseau, Denise M. (1994).

Changing Obligations and the Psychological Contract: A Longitudinal Study. The Academy of Management Journal 37(1): 137-152.

Rousseau, Denise M. (1989). Psychological and implied contracts in organizations. Employee Responsibilities and Rights Journal 2(2): 121-139.

Roy, B. (1981). The optimisation problem formulation: Criticism and overstepping. Journal of the Operational Research Society 32 (6): 427-436.

Runder Tisch Hannover a (2014). Mitarbeiter- und Patientenschutz in der Arztpraxis - Eine Handlungshilfe. ( ${ }^{*}$ Employee and patient protection in the doctor's office - An action aid). [Electronic Article]

Runder Tisch Hannover b (2014). Mitarbeiter- und Patientenschutz in der Arztpraxis - Checklistensammlung. ( ${ }^{*}$ Employee and patient protection in the doctor's office - Checklist collection). [Electronic Article]

Ryan, James; Zwerling, Craig and Orav, Endel John (1992). Occupational Risks Associated with Cigarette Smoking: A Prospective Study. American Journal of Public Health 82(1): 29-32.

Saari, Salli (1981). Ehkäisevän mielenterveystyön teoreettinen perusta. ( ${ }^{*}$ The theoretical basis of preventative mental health work). Psykologia 16(6): 392395.

Saaty, Thomas L. (1980). The Analytic Hierarchy Process: Planning, Priority Setting, Resource Allocation. ed., McGraw-Hill.

Salmón-Mulanovich, Gabriela; Utz, Gregory; Lescano, Andrés G.; Bentzel, David E. and Blazes, David L. (2009). Rapid response to a case of mumps: implications for preventing transmission at a medical research facility. Respuesta rápida a un caso de paperas: implicaciones para la prevención del contagio en una instalación de investigación biomédica. 51(1): 34-38.

Santos, Catarina; Freitas-Magalhães, A. and Castro, Érico (2010). Emotional literacy in health services: Functions, applications and implications. in Freitas-Magalhães, A. Emotional expression: The brain and the face (Vol 2). Porto Portugal, Edições Universidade Fernando Pessoa: 285-299.

Särndal, Carl-Erik; Swensson, Bengt and Wretman, Jan (2003). Model assisted survey sampling. ed. New York, Springer-Verlag.

Saunders, Mark; Lewis, Philip and Thornhill, Adrian (2012). Research methods for business students. 6th ed. Harlow, England ; New York, Pearson.

Scharf, Jessica; Loerbroks, Adrian; Vu-Eickmann, Patricia; Li, Jian; Müller, Andreas and Angerer, Peter (2019). Work-Related Intervention Needs and Potential Occupational Outcomes among Medical Assistants: A Cross-Sectional Study. International journal of environmental research and public health 2019.

Scharf, Jessica; Vu-Eickmann, Patricia; Li, Jian; Müller, Andreas; Wilm, Stefan; Angerer, Peter and Loerbroks, Adrian (2019). Desired improvements of working conditions among medical assistants in Germany: a cross-sectional study. Journal of Occupational Medicine and Toxicology 14.

Schempp, Nadine and Strippel, Harald (2016). Präventionsbericht 2016 - Leistungen 
der gesetzlichen Krankenversicherung: Primärprävention und betriebliche Gesundheitsförderung 2015. ( ${ }^{*}$ Prevention Report 2016 - Statutory Health Insurance Benefits: Primary Prevention and Workplace Health Promotion 2015). Medizinischer Dienst des Spitzenverbandes Bund der Krankenkassen e. V. (MDS), GKV-Spitzenverband

Schirrmann (2017). [Web Page] Der Deming-Kreis. Retrieved from http://www.schirrmann-consulting.de/index.htm?http://www.schirrmannconsulting.de/qualitaetsmanagement/demingkreis.html: 2017-06-17

Schuler, Randall S. and Jackson, Susan E. (1987). Linking Competitive Strategies with Human Resource Management Practices. The Academy of Management Executive (1987-1989) 1(3): 207-219.

Schwandt, T. A. (2001). Dictionary of qualitative inquiry-, Thousand Oaks: Sage. [Encyclopedia]

Schwartz, FW and Walter, U. (2003). Prävention. (The Public Health Book) in Schwartz, B., Busse, Leidl, Raspe, Siegrist, Walter. Das Public Health Buch, Urban und Schwarzenberg: 189-214.

Scott, Michael J. (1989). Management and industrial relations in small firms. ed. [London], [Dept. of Employment].

Seeck, Hannele and Parzefall, Marjo-Riitta (2010). From HRM to psychological contracting - the case of Finnish mobile content producing companies. The International Journal of Human Resource Management 21(15): 2677-2693.

Shackleton, Viv (1995). Business Leadership. ed., Routledge.

Shields, Patricia M. (1998). PRAGMATISM AS PHILOSOPHY OF SCIENCE: A TOOL FOR PUBLIC ADMINISTRATION. Rerearch in Public Adm/n/s/mtian 4: 195-225.

Shipton, H.; Sanders, K.; Atkinson, C. and Frenkel, S. (2015). Sense-giving in health care: the relationship between the HR roles of line managers and employee commitment. Wiley-Blackwell Publishing Ltd. [Electronic Article]

Sisson, Keith (1993). In search of HRM. British Journal of Industrial Relations 31(2): 201.

Sockoll, Ina; Kramer, Ina and Bödeker, Wolfgang (2006). IGA-Report 13 Wirksamkeit und Nutzen betrieblicher Gesundheitsförderung und Prävention - Zusammenstellung der wissenschaftlichen Evidenz 2000 bis 2006. (IGA Report 13 Effectiveness and Benefits of Workplace Health Promotion and Prevention - Compilation of Scientific Evidence 2000 to 2006). BKK Bundesverband

Steinert, W. and Wittmann, A. (2010). Arbeitsschutz in Arztpraxen - Unterschätztes Risiko. ( ${ }^{*}$ Occupational safety in medical practices - underestimated risk). Deutsches Ärzteblatt 107(3): 19-21.

Steinke, M. and Badura, B. (2011). Präsentismus - Ein Review zum Stand der Forschung. ( ${ }^{*}$ Presentism - A review of the state of research). baua: Bundesanstalt für Arbeitsschutz und Arbeitsmedizin

Szecsenyi, Joachim; Goetz, Katja; Campbell, Stephen; Broge, Bjoern; Reuschenbach, Bernd and Wensing, Michel (2011). Is the job satisfaction of primary care team members associated with patient satisfaction? BMJ Quality \& Safety 20 (6): 508-514.

Taylor, F.W. (1911). The Prinicples of Scientific Management-. New York, Dover Publications Inc. [Classical Work] 
Timmermans, S. and Berg, M. (2003). The Gold Standard. The Challenge of Evidence-Based Medicine and Standardization in Health Care. ed. Philadelphia, Temple University Press.

TK (2017). Iss was, Deutschland - TK-Studie zur Ernährung 2017. ( * "Iss was", Germany - TK study on nutrition 2017).

Torrington, Derek; Hall, Laura; Taylor, Stephen and Atkinson, Carol (2017). Human Resource Management. Tenth ed., Pearson Education Limited, Edinburgh.

Tyson, S. (1995). Human resource strategy : towards a general theory of human resource management. ed. London, Pitman Pub.

Tzafrir, Shay S. (2005). The relationship between trust, HRM practices and firm performance. International Journal of Human Resource Management 16(9): 1600-1622.

Ulich, E. (1981). Subjektive Tätigkeitsanalyse als Vorraussetzung autonomieorientierter Arbeitsgestaltung. ( ${ }^{*}$ Subjective activity analysis as a prerequisite for autonomy-oriented work design) in Ulich, F. F. E. Beiträge zur psychologischen Arbeitsanalyse. Bern, Huber: 327-347.

Ulrich, Dave and Smallwood, Norm (2002). Seven up. People Management 8(10).

Vagholkar, Sanjyot; Ng, Jude; Chan, Raymond C.; Bunker, Jeremy M. and Zwar, Nicholas A. (2008). Healthcare workers and immunity to infectious diseases. Australian \& New Zealand Journal of Public Health 32(4): 367-371.

Van De Voorde, Karina; Paauwe, Jaap and Van Veldhoven, Marc (2012). Employee Well-being and the HRM-Organizational Performance Relationship: A Review of Quantitative Studies. International Journal of Management Reviews 14(4): 391-407.

van Strien, Tatjana and Koenders, Paul (2010). How do physical activity, sports, and dietary restraint relate to overweight-associated absenteeism? Journal of Occupational and Environmental Medicine 52(9): 858-864.

Vester, Frederic (2009). Die Kunst vernetzt zu denken: Ideen und Werkzeuge für einen neuen Umgang mit Komplexität: Ideen und Werkzeuge für einen neuen Umgang mit Komplexität. Ein Bericht an den Club of Rome. ed. ( ${ }^{*}$ The art of networking: ideas and tools for a new approach to complexity: ideas and tools for a new approach to complexity. A report to the Club of Rome). dtv.

Vicente-Herrero, M. T.; Torre, M. V. Ramírez Iñiguez de la; García, L. M. Capdevila; López-González, A. A. and García, M. J. Terradillos Trabajando juntos: Las alergias en salud laboral. Aspectos preventivos. Allergies in occupational health. Prevention aspects (English).

Vu-Eickmann, Patricia and Loerbroks, Adrian (2017). Psychosoziale Arbeitsbedingungen und Versorgungsqualität: Ergebnisse einer qualitativen Studie unter Medizinischen Fachangestellten (MFAs). Das Gesundheitswesen.

Walter; Plaumann, M.; Dubben, S.; Nöcker, G. and Kliche, T. (2011). Gesundheitsökonomische Evaluationen in der Prävention und Gesundheitsförderung. ( ${ }^{*}$ Health economic evaluations in prevention and health promotion). Prävention und Gesundheitsförderung 6(2): 94-101.

Walter, Uta (2007) [Thesis]: Qualitätsentwicklung durch Standardisierung - am Beispiel des Betrieblichen Gesundheitsmanagements. ( ${ }^{*}$ Quality development through standardization - using the example of workplace 
health management). Universität Bielefeld

Wang, Yumei; Zhu, Zhen and Cong, Qing (2009). An Analysis on the Knowledge Workers' Turnover: A Psychological Contract Perspective. International Journal of Business and Management 3(3).

Warr, Peter; Cook, John and Wall, Toby (1979). Scales for the measurement of some work attitudes and aspects of psychological well-being. Journal of Occupational Psychology 52(2): 129-148.

Wason, Peter (1960). On the failure to eliminate hypotheses in a conceptual task. Journal of Experimental Psychology 12: 129-140.

Waters, T.; Collins, J.; Galinsky, T. and Caruso, C. (2006). NIOSH research efforts to prevent musculoskeletal disorders in the healthcare industry. Orthopaedic Nursing 25(6): 380-389.

Webendörfer, Stefan (2011). Prevention of bowel cancer.

Whitener, E. M. (1997). The Impact of Human Resource Activities on Employee Trust. HUMAN RESOURCE MANAGEMENT REVIEW 7(4): 389-404.

Whittlestone, Jess (2017) [Thesis]: The importance of making assumptions: why confirmation is not necessarily a bias. University of Warwick

Wilkinson, Adrian (1999). Employment relations in SMEs. Employee Relations 21(3): 206.

Wilms, Falko E. P. (2012). Wirkungsgefüge - Einsatzmöglichkeiten und Grenzen in der Unternehmensführung. ed. ( ${ }^{*}$ Impact structures - possible uses and limits in corporate governance). Haupt Verlag, Bern.

Wood, Stephen and de Menezes, Lilian (1998). High commitment management in the UK: Evidence from the workplace industrial relations survey, and employers' manpower and skills practices survey. Human Relations 51(4).

Wood, Stephen J. and Wall, Toby D. (2007). Work enrichment and employee voice in human resource management-performance studies. The International Journal of Human Resource Management 18(7): 1335-1372.

Wright, Patrick M. and Boswell, Wendy Robyn (2002). Desegregating HRM : a review and synthesis of micro and macro human resource management researchlthaca, N.Y., Center for Advanced Human Resource Studies, Cornell University. [Electronic Book]

Wright, Patrick M. and McMahan, Gary C. (1992). Theoretical Perspectives for Strategic Human Resource Management. Journal of Management 18(2): 295-320.

Wright, Patrick M.; McMahan, Gary C. and McWilliams, Abagail (1994). Human resources and sustained competitive advantage: a resource-based perspective. The International Journal of Human Resource Management 5(2): 301-326.

Zangemeister, Christof (2014). Nutzwertanalyse in der Systemtechnik: Eine Methodik zur multidimensionalen Bewertung und Auswahl von Projektalternativen. 5th ed. ( ${ }^{*}$ Value analysis in systems engineering: $A$ methodology for multidimensional evaluation and selection of project alternatives). Hamburg, Zangemeister \& Partner.

Zelfel, R. C.; Alles, T. and Weber, A. (2011). Health Management in Small and Medium-Sized Enterprises - Results of a Representative Survey. Das Gesundheitswesen 73(9): 515-519. 


\section{Appendices}

\section{Appendix 1: Abbreviations}

AGnES

AHP

ÄApprO

AMO

ANP

ArbSchG

ASiG

BÄK

BARMER GEK

BDSG

BetrVG

BG

BGB

BGW

BIBB

BMG

BSC

BZgA

destatis
Community-based, e-health-assisted, systemic intervention to reduce physicians' workloads (German: Arztentlastende, Gemeindenahe, E-Healthgestützte, Systemische Intervention)

Analytic hierarchy process

Medical Licensure Act for Physicians (German: Approbationsordnung für Ärzte)

Ability (A), Motivation (M) and Opportunity (O)

Analytic network process

German Occupational Safety and Health Act (German: Arbeitsschutzgesetz)

German Occupational Safety Act (German: Arbeitssicherheitsgesetz)

German Medical Association (German: Bundesärztekammer)

Name of a great health insurance company (German:

Barmer Ersatzkasse und ehemals Gmünder Ersatzkasse)

German law about data protection (German: Bundesdatenschutzgesetz)

Works Constitution Act (German: Betriebsverfassungsgesetz)

German employer's liability insurance association (German:

Berufsgenossenschaft)

German Civil Code (German: Bürgerliches Gesetzbuch)

Liability Insurance Association for Health Service and Welfare Work (German: Berufsgenossenschaft für Gesundheit und Wohlfahrtspflege)

Federal Institute for Vocational Education and Training (German: Bundesinstitut für Berufsbildung)

Federal Ministry of Health (German: Bundesministerium für Gesundheit)

Balanced Scorecard

Federal Centre for Health Education (German: Bundeszentrale für gesundheitliche Aufklärung)

Federal Statistical Office (German: Statistisches Bundesamt) 


\begin{tabular}{|c|c|}
\hline DGAUM & $\begin{array}{l}\text { German Society of Occupational and Environmental Medicine } \\
\text { (German: Deutsche Gesellschaft für Arbeitsmedizin und Um- } \\
\text { weltmedizin e. V.) }\end{array}$ \\
\hline DGUV & $\begin{array}{l}\text { German Social Accident Insurance (German: Deutsche Gesetz- } \\
\text { liche Unfallversicherung) }\end{array}$ \\
\hline DissOnline & $\begin{array}{l}\text { Thesis Online Service of DNB (German: Dissertationen } \\
\text { Online) }\end{array}$ \\
\hline DNB & $\begin{array}{l}\text { German National Library (German: Deutsche Nationalbibli- } \\
\text { othek) }\end{array}$ \\
\hline DVD & Digital Versatile Disc \\
\hline ENT & Ear-nose-threat \\
\hline EPA & $\begin{array}{l}\text { European Practice Assessment (German: Europäisches } \\
\text { Praxisassessment) }\end{array}$ \\
\hline EStG & Income Tax Act (German: Einkommensteuergesetz) \\
\hline EThOS & Electronic Thesis Online Service \\
\hline G-BA & $\begin{array}{l}\text { Federal Joint Committee of Insurance Companies and Phy- } \\
\text { sicians (German: Gemeinsamer Bundesausschuss) }\end{array}$ \\
\hline GewStG & German Trade Tax Act (German: Gewerbesteuergesetz) \\
\hline GKV & $\begin{array}{l}\text { Statutory health insurance (German: Gesetzliche Kranken- } \\
\text { versicherung) }\end{array}$ \\
\hline GOÄ & $\begin{array}{l}\text { German Medical fee schedule (German: Gebührenordnung } \\
\text { für Ärzte) }\end{array}$ \\
\hline GP & General practitioner \\
\hline HIV & $\begin{array}{l}\text { Human immunodeficiency virus (German: Humanes Im- } \\
\text { mundefizienz-Virus) }\end{array}$ \\
\hline HPV & $\begin{array}{l}\text { Human papillomaviruses (German: Humane Papillo- } \\
\text { maviren) }\end{array}$ \\
\hline HPWP & High Performance Work Practices \\
\hline HR & Human Resource \\
\hline HRM & Human Resource Management \\
\hline $\mathrm{HzV}$ & $\begin{array}{l}\text { Family doctor-centred health care (German: Hausarztzen- } \\
\text { trierte Versorgung) }\end{array}$ \\
\hline ICD10 & $\begin{array}{l}\text { International Statistical Classification of Diseases and Re- } \\
\text { lated Health Problems (Revision 10) }\end{array}$ \\
\hline IGA & $\begin{array}{l}\text { Health and Work Initiative (German: Initiative Gesundheit } \\
\text { und Arbeit) }\end{array}$ \\
\hline IGeL & $\begin{array}{l}\text { Individual Health Services (German: Individuelle Gesund- } \\
\text { heits-Leistungen) }\end{array}$ \\
\hline KeGL & Competence Development of Health Professionals in the \\
\hline
\end{tabular}


KMK

KTQ

KV

KVB

MACBETH

MAUT

MCDA

MFA

MQLD

MVZ

OHS

OP

$\mathrm{OSH}$

PKV

PTA

PrävG

QEP

QM

QM-RL

REC

RKI

SD
Context of Lifelong Learning (German: Kompetenzentwicklung von Gesundheitsfachpersonal im Kontext des Lebenslangen Lernens)

Standing Conference of the Ministers of Education and Cultural Affairs of the Länder in the Federal Republic of Germany (German: Kultusministerkonferenz - Kurzform für Ständige Konferenz der Kultusminister der Länder in der Bundesrepublik Deutschland)

Cooperation for transparency and quality in health care (German: Kooperation für Transparenz und Qualität im Gesundheitswesen)

Association of Statutory Health Insurance Physicians (German: Kassenärztliche Vereinigung)

Bavarian Association of Statutory Health Insurance Physicians (German: Kassenärztliche Vereinigung Bayern)

Measuring Attractiveness by a Categorical Based Evaluation Technique

Multi-attribute utility theory

Multi-criteria decision analysis

Medical Assistent (German: Medizinische Fachangestellte)

Quality Network Langen Dreieich (German: Medizinisches Qualitätsnetz Langen Dreieich)

Medical Service Centres (German: Medizinisches Versorgungszentrum)

Occupational Health and Security

Organizational Performance

Occupational Safety and Health

Private health insurance funds

Pharmaceutical technician (German: Pharmazeutischtechnische Assistenten)

Präventionsgesetz (Prevention Act)

Quality and development in surgeries (German: Qualität und Entwicklung in Praxen)

Quality management (German: Qualitätsmanagement)

Quality management guidelines of G-BA (German: Qualitätsmanagement-Richtlinie)

Record of ethical conduct

Robert Koch Institute (German: Robert Koch Institut)

Standard deviation 
SGB

SHI

SMC

SME

STIKO

TBE

TK

UK

UStG

VeraH

VVG

WB

WHO

WHP

ZFA
German social security code (German: Sozialgesetzbuch) Social health insurance

Small and medium sized companies

Small and medium sized enterprises

Standing Committee on Vaccination at the Robert Koch Institute (German: Ständige Impfkommission am RKI)

Tick-borne encephalitis (TBE)

Name of a great health insurance company (German: Technikerkrankenkasse)

United Kingdom

Value Added Tax Act (German: Umsatzsteuergesetz)

Care assistant in the domestic practice (German: Versorgungsassistentin in der Hausarztpraxis)

German Insurance Contract Act (German: Versicherungsvertragsgesetz)

Well-being

World Health Organization

Workplace health promotion

Dental (chairside) assistant (German: Zahnmedizinische Fachangestellte) 


\section{Appendix 2: Results of literature research "Personal health potentials"}

\begin{tabular}{|l|l|l|}
\hline Title & Relevant & Explanation \\
\hline $\begin{array}{l}\text { Workplace interventions to prevent musculoskeletal } \\
\text { and visual symptoms and disorders among computer } \\
\text { users: a systematic review (Brewer, Van Eerd et al., } \\
2006)\end{array}$ & Yes & $\begin{array}{l}\text { Systematic evaluation } \\
\text { of existing studies }\end{array}$ \\
\hline $\begin{array}{l}\text { A study of the experiences of participants following } \\
\text { attendance at a workshop on methods to prevent or } \\
\text { reduce work-related musculoskeletal disorders } \\
\text { amongst sonographers (Gibbs and Young, 2011) }\end{array}$ & No & $\begin{array}{l}\text { The focus is to narrow } \\
\text { only on sonographers }\end{array}$ \\
\hline $\begin{array}{l}\text { NIOSH research efforts to prevent musculoskeletal } \\
\text { disorders in the healthcare industry (Waters, Collins et } \\
\text { al., 2006) }\end{array}$ & Partly & $\begin{array}{l}\text { The focus is on the } \\
\text { healthcare sector but } \\
\text { on big organisations }\end{array}$ \\
\hline $\begin{array}{l}\text { A randomised controlled trial on whether a participa- } \\
\text { tory ergonomics intervention could prevent musculo- } \\
\text { skeletal disorders.(Haukka, Leino-Arjas et al., 2008) }\end{array}$ & No & $\begin{array}{l}\text { Only focused on } \\
\text { kitchen workers }\end{array}$ \\
\hline $\begin{array}{l}\text { How we prevent prevention of musculoskeletal disor- } \\
\text { ders in the workplace (Melhorn and Gardner, 2004) }\end{array}$ & Yes & $\begin{array}{l}\text { Example for costs of } \\
\text { employee illness }\end{array}$ \\
\hline $\begin{array}{l}\text { Elimination of manual patient handling to prevent work- } \\
\text { related musculoskeletal disorders (Bunke, 2008) }\end{array}$ & No & $\begin{array}{l}\text { No more information } \\
\text { available }\end{array}$ \\
\hline $\begin{array}{l}\text { Regulatory actions to prevent work-related musculo- } \\
\text { skeletal disorders--the use of research-based expo- } \\
\text { sure limits (Fallentin, 2003) }\end{array}$ & No & $\begin{array}{l}\text { Insufficient infor- } \\
\text { mation }\end{array}$ \\
\hline $\begin{array}{l}\text { Clinical update in musculoskeletal medicine. "Joint } \\
\text { motion" gaining momentum: worldwide effort to re- } \\
\text { search, manage, and prevent musculoskeletal disor- } \\
\text { ders (N., 2001) }\end{array}$ & No & $\begin{array}{l}\text { Insufficient infor- } \\
\text { mation }\end{array}$ \\
\hline $\begin{array}{l}\text { A test of two training interventions to prevent work- } \\
\text { related musculoskeletal disorders of the upper extremi- } \\
\text { ty (Faucett, Garry et al., 2002) }\end{array}$ & No & $\begin{array}{l}\text { Very focused small } \\
\text { group }\end{array}$ \\
\hline $\begin{array}{l}\text { Position statement: elimination of manual patient han- } \\
\text { dling to prevent work-related musculoskeletal disorders } \\
\text { (2004) }\end{array}$ & No & Focus on nursing \\
\hline
\end{tabular}

Results of search for 'prevent work-related musculoskeletal disorders'

\begin{tabular}{|l|l|l|}
\hline Title & Relevant & \multicolumn{1}{|c|}{ Explanation } \\
\hline $\begin{array}{l}\text { Work-related Violence and Incident Use of Psy- } \\
\text { chotropic (Madsen, Burr et al., 2011) }\end{array}$ & No & Insufficient information \\
\hline $\begin{array}{l}\text { Mental Health and Work: Issues and Perspectives } \\
\text { (Morrow, Verins et al., 2002) }\end{array}$ & No & Focus on Australia \\
\hline $\begin{array}{l}\text { Improving responses to depression and related } \\
\text { disorders: Evaluation of an innovative, general, } \\
\text { mental health care workers training program. } \\
\text { (Graham, Julian et al., 2010) }\end{array}$ & Partly & Focus on Australia \\
\hline $\begin{array}{l}\text { Staying Sane May Be Easier Than You Think. } \\
\text { (cover story) (Cloud, 2009) }\end{array}$ & No & $\begin{array}{l}\text { Focus on private life. Not } \\
\text { academic }\end{array}$ \\
\hline $\begin{array}{l}\text { Preventive Interventions under Managed Care: } \\
\text { Mental Health and Substance Abuse Services. } \\
\text { Special Report. (Dorfman, 2000) }\end{array}$ & No & $\begin{array}{l}\text { Too regional with a focus } \\
\text { on USA }\end{array}$ \\
\hline $\begin{array}{l}\text { Emotional literacy in health services: Functions, } \\
\text { applications and implications (Santos, Freitas- } \\
\text { Magalhães et al., 2010) }\end{array}$ & No & only for the nurses \\
\hline
\end{tabular}




\begin{tabular}{|l|l|l|}
\hline Title & Relevant & \multicolumn{1}{|c|}{ Explanation } \\
\hline $\begin{array}{l}\text { The theoretical basis of preventive mental health } \\
\text { work (Saari, 1981) }\end{array}$ & Yes & $\begin{array}{l}\text { Very theoretical and more } \\
\text { general prevention and not } \\
\text { work prevention }\end{array}$ \\
\hline $\begin{array}{l}\text { National Association for Mental Hygiene: its ori- } \\
\text { gins, purposes, and personnel (National } \\
\text { Association for Mental Health, 1929) }\end{array}$ & No & $\begin{array}{l}\text { Information about a psy- } \\
\text { chological foundation }\end{array}$ \\
\hline
\end{tabular}

Results of search for 'prevent mental disorder'

\begin{tabular}{|l|l|l|}
\hline Title & Relevant & \multicolumn{1}{|c|}{ Explanation } \\
\hline $\begin{array}{l}\text { Allergies in occupational health. Prevention as- } \\
\text { pects (Vicente-Herrero, Torre et al.) }\end{array}$ & No & Not available in English \\
\hline $\begin{array}{l}\text { Cost-benefit analysis in occupational health: a } \\
\text { comparison of intervention scenarios for occupa- } \\
\text { tional asthma and rhinitis among bakery workers. } \\
\text { (Meijster, van Duuren-Stuurman et al., 2011) }\end{array}$ & partly & $\begin{array}{l}\text { The focus is on bakery } \\
\text { worker and not on the ser- } \\
\text { vice sector }\end{array}$ \\
\hline $\begin{array}{l}\text { Occupational respiratory diseases in South Afri- } \\
\text { ca-results (Esterhuizen, Hnizdo et al., 2001) }\end{array}$ & No & $\begin{array}{l}\text { Irrelevant geographic re- } \\
\text { gion }\end{array}$ \\
\hline $\begin{array}{l}\text { Occupational asthma as identified by the Surveil- } \\
\text { lance of Work-related and Occupational Respira- } \\
\text { tory Diseases programme in South Africa } \\
\text { (Hnizdo, Esterhuizen et al., 2001) }\end{array}$ & No & $\begin{array}{l}\text { Irrelevant geographic re- } \\
\text { gion }\end{array}$ \\
\hline $\begin{array}{l}\text { Occupational medicine in the Slovene area } \\
\text { (Bilban, 2005) }\end{array}$ & No & $\begin{array}{l}\text { Many relevant figures but } \\
\text { for Slovenia and not Ger- } \\
\text { many }\end{array}$ \\
\hline $\begin{array}{l}\text { Occupational contact dermatitis I: incidence and } \\
\text { return to work pressures (Emmett, 2002) }\end{array}$ & No & $\begin{array}{l}\text { Focus on dermatitis, irrele- } \\
\text { vant geographic region }\end{array}$ \\
\hline $\begin{array}{l}\text { Respiratory diseases in confined non-industrial } \\
\text { working environments (Muzi, Murgia et al., 2006) }\end{array}$ & No & Medical focus \\
\hline $\begin{array}{l}\text { Chemical hazards in radiology (Byrns, Palatianos } \\
\text { et al., 2000) }\end{array}$ & No & Focus on physicians \\
\hline $\begin{array}{l}\text { Occurrence and causes of occupational asthma } \\
\text { in South Africa--results from SORDSA's Occupa- } \\
\text { tional Asthma Registry, 1997-1999 (Esterhuizen, } \\
\text { Hnizdo et al., 2001) }\end{array}$ & No & $\begin{array}{l}\text { Irrelevant geographic re- } \\
\text { gion }\end{array}$ \\
\hline
\end{tabular}

Results of search for 'prevent respiratory diseases'

\begin{tabular}{|l|l|l|}
\hline Title & Relevant & Explanation \\
\hline $\begin{array}{l}\text { Occupational vaccination of health care workers: } \\
\text { Uptake, attitudes and potential solutions } \\
\text { (Vagholkar, Ng, Chan, Bunker, \& Zwar, 2008) }\end{array}$ & Yes & \\
\hline $\begin{array}{l}\text { Knowledge, attitudes and beliefs of health care } \\
\text { workers towards influenza vaccination. (Mytton, } \\
\text { O'Moore et al., 2013) }\end{array}$ & partly & $\begin{array}{l}\text { Good study about the think- } \\
\text { ing of healthcare workers } \\
\text { about vaccines }\end{array}$ \\
\hline Flu -- to jab or not to jab? (Hall, 2013) & partly & \\
\hline $\begin{array}{l}\text { Sustained low influenza vaccination rates in US } \\
\text { healthcare workers (Caban-Martinez, Lee et al., } \\
\text { 2010) }\end{array}$ & No & Irrelevant geographic region \\
\hline $\begin{array}{l}\text { Containment of Polioviruses After Eradication } \\
\text { and OPV Cessation: Characterizing Risks to } \\
\text { Improve Management (Dowdle, Van der Avoort } \\
\text { et al., 2006) }\end{array}$ & No & $\begin{array}{l}\text { Not focused on occupational } \\
\text { medicine }\end{array}$ \\
\hline
\end{tabular}




\begin{tabular}{|l|l|l|}
\hline Title & Relevant & \multicolumn{1}{|c|}{ Explanation } \\
\hline $\begin{array}{l}\text { Rapid response to a case of mumps: implications } \\
\text { for preventing transmission at a medical research } \\
\text { facility. (Salmón-Mulanovich, Utz et al., 2009) }\end{array}$ & No & $\begin{array}{l}\text { Not focused on occupational } \\
\text { medicine }\end{array}$ \\
\hline Immunisation policy (Dunn, 2008) & Yes & \\
\hline A safe pair of hands (Hargreaves, 2008) & No & $\begin{array}{l}\text { The content is about training } \\
\text { for nurse }\end{array}$ \\
\hline $\begin{array}{l}\text { Improving influenza immunization uptake in } \\
\text { healthcare workers (Preece, 2012) }\end{array}$ & Yes & \\
\hline $\begin{array}{l}\text { Healthcare workers and immunity to infectious } \\
\text { diseases (Vagholkar, Ng et al., 2008) }\end{array}$ & No & Irrelevant geographic region \\
\hline $\begin{array}{l}\text { Specific immunization issues in the occupational } \\
\text { health setting. (Baxter, 2007) }\end{array}$ & No & $\begin{array}{l}\text { Irrelevant geographic region } \\
\text { and focus on legal issues }\end{array}$ \\
\hline $\begin{array}{l}\text { Hepatitis B in Moroccan health care workers } \\
\text { (Djeriri, Laurichesse et al., 2008) }\end{array}$ & No & \\
\hline
\end{tabular}

Results of database search in Ebsco for 'vaccines' 


\section{Appendix 3: Original (German) questionnaire}

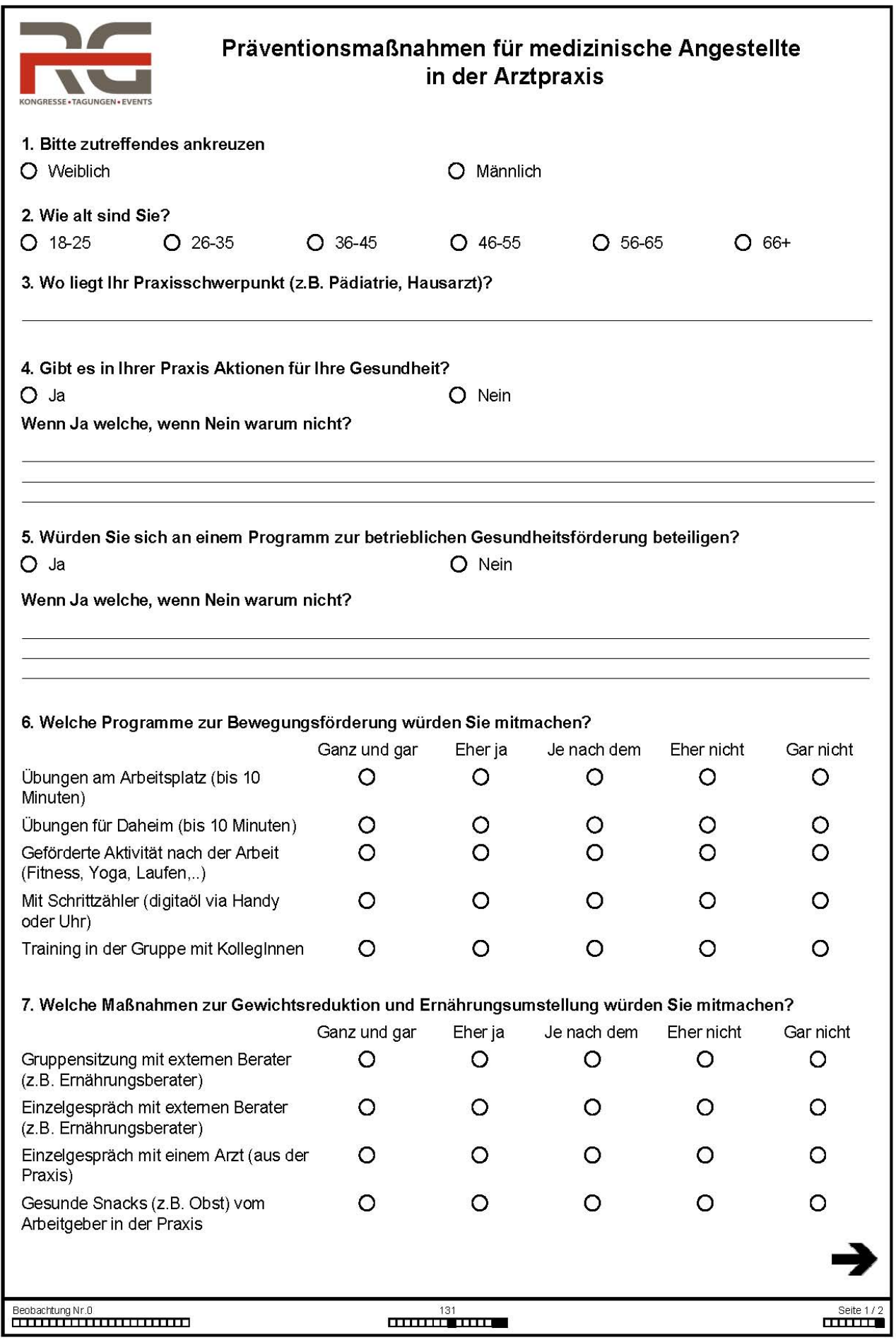




\section{Präventionsmaßnahmen für medizinische Angestellte in der Arztpraxis}

8. Lassen Sie sich grundsätzlich regelmäßig privat impfen (z.B. Grippe)?

$\bigcirc \mathrm{Ja} \quad O$ Nein

Wenn Ja welche Impfungen, wenn Nein warum nicht?

9. Würden Sie sich in der Praxis Ihres Arbeitgebers impfen lassen?

$\bigcirc \mathrm{Ja}$

Nein

Wenn Ja welche Impfung, wenn Nein warum nicht?

10. Lassen Sie Ihren Impfstatus regelmäßig prüfen?

O Ja

Nein

Wenn Nein warum nicht?

Bitte geben Sie den ausgefüllten Fragebogen an der Anmeldung ab.

VIELEN DANK für Ihre Mithilfe! 


\section{Appendix 4: Results of the questionnaires}

\section{$\underline{\text { Prevention activities for employees in general practitioners' surgeries }}$}

Returned questionnaires 203

$\begin{array}{ll}\text { Completed questionnaires } & 194\end{array}$

Gender

Female 194

Male 0

Age

$\begin{array}{ll}18-25 & 59\end{array}$

$26-35 \quad 34$

$36-45$

$46-55 \quad 35$

$\begin{array}{ll}56-65 & 15\end{array}$

$66+\quad 0$

No answer 2

What kind of general practitioner surgery you work for?

$\begin{array}{lrr}\text { General practitioner } & \mathbf{1 6 5} & \mathbf{8 5 \%} \\ \text { Gynaecologist } & 9 & 5 \% \\ \text { Other } & 6 & 3 \% \\ \text { No answer } & 14 & 7 \%\end{array}$

Are there activities for health management in your general practitioner surgery?

Yes

No

Sum answer

No answer

If yes what, if no why?

Check-up, Vaccines, nutrition advise

Would you join a health prevention program?

Yes

No

Sum answer

No answer

If yes what, if no why?

Healthy nutrition, fitness

$\begin{array}{rrr}45 & 26 & \% \\ 129 & 74 & \% \\ 174 & 100 & \%\end{array}$

$20 \quad 10 \quad \%$

$97 \quad 68 \quad \%$

$46 \quad 32 \%$

$143 \quad 100 \%$

$5126 \%$ 
Which programs for activity promotion you would join?

Training at the workplace (max. $10 \mathrm{~min}$ )

Training at home (max. $10 \mathrm{~min})$

Sponsored activity after work (Fitness, Yoga, etc.)

With a counter for the steps or a fitness watch

Training within a group of colleagues

Training at the workplace (max. $10 \mathrm{~min})$

Training at home (max. $10 \mathrm{~min})$

Sponsored activity after work (Fitness, Yoga, etc)

With a counter for the steps or a fitness watch

Training within a group of colleagues

Training at the workplace (max. $10 \mathrm{~min}$ )

Training at home (max. $10 \mathrm{~min})$

Sponsored activity after work (Fitness, Yoga, etc)

With a counter for the steps or a fitness watch

Training within a group of colleagues

\begin{tabular}{|c|c|c|c|c|c|}
\hline yes & $\begin{array}{l}\text { more } \\
\text { yes }\end{array}$ & depends & more no & no & sum \\
\hline 43 & 38 & 52 & 30 & 17 & 180 \\
\hline 26 & 64 & 44 & 34 & 11 & 179 \\
\hline 49 & 62 & 38 & 23 & 13 & 185 \\
\hline 36 & 51 & 31 & 40 & 24 & 182 \\
\hline 25 & 46 & 54 & 36 & 20 & 181 \\
\hline
\end{tabular}

more
yes yes

$24 \% \quad 21 \%$

$15 \% \quad 36 \%$

depends more no no sum

$26 \%$

$20 \%$

$14 \%$

$34 \%$

$28 \%$

$25 \%$

Yes No

$45 \% \quad 26 \%$

$50 \% \quad 25 \%$

$60 \% \quad 19 \%$

$48 \% \quad 35 \%$

$39 \% \quad 31 \%$

Which activities for weight reduction and change of nutrition would you support?

Would you join a group session with an external consultant?

Would you join a single session with an external consultant?

Would you join a session with a medical physician from your medical office?

Would you like to get healthy snacks/food at work?

Would you join a group session with an external consultant?

Would you join a single session with an external consultant?

Would you join a session with a medical physician from your medical office?

Would you like to get healthy snacks/food at work?
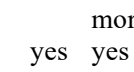

20

26

15

106

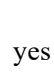

more

yes yes

$11 \%$

$14 \%$

$9 \%$

$58 \%$

more depends more no no sum

40

58

31

47

47

$22 \%$

depends more no no sum

$32 \%$

$27 \%$

$25 \%$

$23 \% \quad 16 \%$

$15 \% \quad 13 \%$

$18 \%$

$24 \%$

$30 \% \quad 20 \%$

$26 \%$

$8 \%$

$4 \% \quad 4 \%$

Would you join a group session with an external consultant?

$34 \% \quad 40 \%$

$47 \% \quad 28 \%$

$26 \% \quad 50 \%$

$84 \% \quad 8 \%$

Do you regularly use vaccine in your private life? 


$\begin{array}{lrrr}\text { Yes } & & \text { No } & \\ & 145 & 37 & 182\end{array}$

Please explain why or why not.

Polio

Flu

Tetanus

FMSE

Would you join a vaccine program at work?

\begin{tabular}{|c|c|c|}
\hline Yes & No & \\
\hline 171 & 12 & \\
\hline $93 \%$ & $7 \%$ & \\
\hline $\begin{array}{l}\text { No } \\
\text { answer }\end{array}$ & 11 & \\
\hline Yes & No & Sum \\
\hline 163 & 20 & \\
\hline $89 \%$ & $11 \%$ & \\
\hline
\end{tabular}




\section{Appendix 5: The structured interview guide for the general practitioners}

\section{$\underline{\text { Classification and characteristics of one's own practice }}$}

1.1 Is your general practitioner's surgery in the city centre, on the outskirts or in the country?

1.2 How many MFAs or medical assistants do you employ full-time or part-time and what is your age structure?

1.3 Does your surgery have a special range of services (acupuncture, travel medicine...)?

1.4 Does your surgery offer primary prevention services?

1.5 Take the MFAs tasks of primary prevention (vaccination, movement training, nutritional advice, stress avoidance, addiction prevention...)? Do you intend to delegate these tasks?

1.6 Do the MFAs take on home-visit tasks?

1.7 Are there any disturbances in the practice organization due to sickness-related absence? Are absences systematically recorded and evaluated?

1.8 What health-promoting measures are implemented in your surgery?

$\underline{\text { Reconciliation of the list of priorities with your own ideas }}$

My study, among other things, is about ten primary prevention measures that are to be investigated at MFAs in general practitioners' surgeries. The study will propose a standard recommendation for introduction.

2.1 For each primary prevention measure, you will find an entry for 
- Acceptance by the MFAs: Here I am interested in your assessment of the average acceptance by your employees (well accepted/accepted/neutral/not accepted/accepted)

- Effectiveness after evaluation of studies: Here I am interested in your assessment from a medical point of view regarding the effective avoidance of absence days. (High/medium/low/do not know)

- Cost level by experience: Here you will find the result of my research on estimation of the expected costs. How do you estimate the cost level? (rather higher/goes $\mathrm{OK} /$ rather lower).

\begin{tabular}{|c|c|c|c|c|c|c|}
\hline \multirow[t]{2}{*}{ Measure } & \multirow{2}{*}{$\begin{array}{c}\text { Assumed } \\
\text { Acceptance }\end{array}$} & \multicolumn{2}{|c|}{ Effectiveness } & \multicolumn{2}{|c|}{ Cost level } & \multirow[t]{2}{*}{ Own. } \\
\hline & & acc. Study & physician & Estimate & physician & \\
\hline $\begin{array}{l}\text { Workplace exercises } \\
\text { (up to } 10 \text { minutes) }\end{array}$ & & $\begin{array}{l}\text { Low } \\
\text { (33) }\end{array}$ & & $\begin{array}{l}\text { Low } \\
\text { (75) }\end{array}$ & & \\
\hline $\begin{array}{l}\text { Exercises for home } \\
\text { (up to } 10 \text { minutes) }\end{array}$ & & $\begin{array}{l}\text { medium } \\
(50)\end{array}$ & & $\begin{array}{c}\text { Very low } \\
(100)\end{array}$ & & \\
\hline $\begin{array}{l}\text { Sponsored activity } \\
\text { after work (fitness, } \\
\text { yoga, running } \\
\text { group...) }\end{array}$ & & $\begin{array}{l}\text { Medium } \\
\quad(50)\end{array}$ & & $\begin{array}{l}\text { Low } \\
\text { (75) }\end{array}$ & & \\
\hline $\begin{array}{l}\text { Training with step } \\
\text { counter (digital via } \\
\text { mobile phone or } \\
\text { clock) }\end{array}$ & & $\begin{array}{l}\text { Medium } \\
\text { (66) }\end{array}$ & & $\begin{array}{l}\text { Low } \\
\text { (75) }\end{array}$ & & \\
\hline $\begin{array}{l}\text { Training in the group } \\
\text { with colleagues }\end{array}$ & & $\begin{array}{l}\text { Very low } \\
\text { (16) }\end{array}$ & & $\begin{array}{l}\text { Medium } \\
\text { (62) }\end{array}$ & & \\
\hline $\begin{array}{l}\text { Group meeting with } \\
\text { external consultants } \\
\text { (e.g. nutritionist) }\end{array}$ & & $\begin{array}{l}\text { Medium } \\
\quad(42)\end{array}$ & & $\begin{array}{c}\text { Very high } \\
25)\end{array}$ & & \\
\hline $\begin{array}{l}\text { Individual discussion } \\
\text { with external consult- } \\
\text { ant (e.g. nutritionist) }\end{array}$ & & $\begin{array}{l}\text { Medium } \\
\quad(42)\end{array}$ & & $\begin{array}{c}\text { Very high } \\
25)\end{array}$ & & \\
\hline $\begin{array}{l}\text { Individual conversa- } \\
\text { tion with a physician }\end{array}$ & & $\begin{array}{l}\text { Medium } \\
\text { (42) }\end{array}$ & & $\begin{array}{l}\text { Medium } \\
\text { (37) }\end{array}$ & & \\
\hline
\end{tabular}




\begin{tabular}{|l|c|c|c|c|c|c|}
\hline Measure & \multirow{2}{*}{$\begin{array}{c}\text { Assumed } \\
\text { Acceptance }\end{array}$} & \multicolumn{2}{|c|}{ Effectiveness } & \multicolumn{2}{|c|}{ Cost level } & \multirow{2}{*}{ Own. } \\
\cline { 3 - 6 } & & acc. Study & physician & Estimate & physician & \\
\hline & & & & & & \\
\hline & & $\begin{array}{c}\text { Medium } \\
(58)\end{array}$ & & $\begin{array}{c}\text { Medium } \\
(50)\end{array}$ & & \\
\hline & & $\begin{array}{c}\text { Very high } \\
(100)\end{array}$ & & $\begin{array}{c}\text { Very low } \\
(100)\end{array}$ & & \\
\hline
\end{tabular}

Comments on individual measures

2.2 Do you believe that measures are lacking?

2.3 When deciding on the measures, how would you rate the criteria of acceptance, effectiveness and cost? (Suppose you have 100 points to allocate.) Are there more decision criteria?

2.4 If you introduce health management measures, would you ask for acceptance by the MFAs? (Or did you do this if measures were introduced?) If rather no, reasons for this?

2.5 The elaboration of one practice-specific introductory concept requires time. How many hours would you quote? - or what time savings can be made by using the standard recommendation?

2.6 In case of significant deviations from the proposed concept, would you use software (e.g. Excel) to prepare your specific decision?

$\underline{\text { Influencing factors to control productivity and performance }}$

3.1 What importance do you attach to the following levers for reducing absent days:

- health promoting measures for MFAs 
- work organization in the surgery

- profile/job design of the MFAs

- leadership quality/competence

3.2 In your opinion, what other starting points and measures to reduce absent days should be taken more into account in your surgery?

Implementation of health management in one's own surgery

4.1 In which order you would realize the four levers (of question 3.1)?

4.2 In your view, is there any reason why a general practitioner's surgery should introduce a health management system in comparison with other organisations and companies?

4.3 How do you practice health management measures so far and what significance do you attach to it?

- Not at all (name possible reasons.)

- As individual projects (name specific examples.)

- As a long-term initiative (how is success controlled?)

- Integrated in quality management (with what importance?)

4.4 What significance do concepts such as "healthy leadership", "health culture in the physician's office" or "Health competence MFA" (all taken from the training programs of medical chambers) have for you? 
The target system of health management in general practitioners' surgeries as causeeffect network

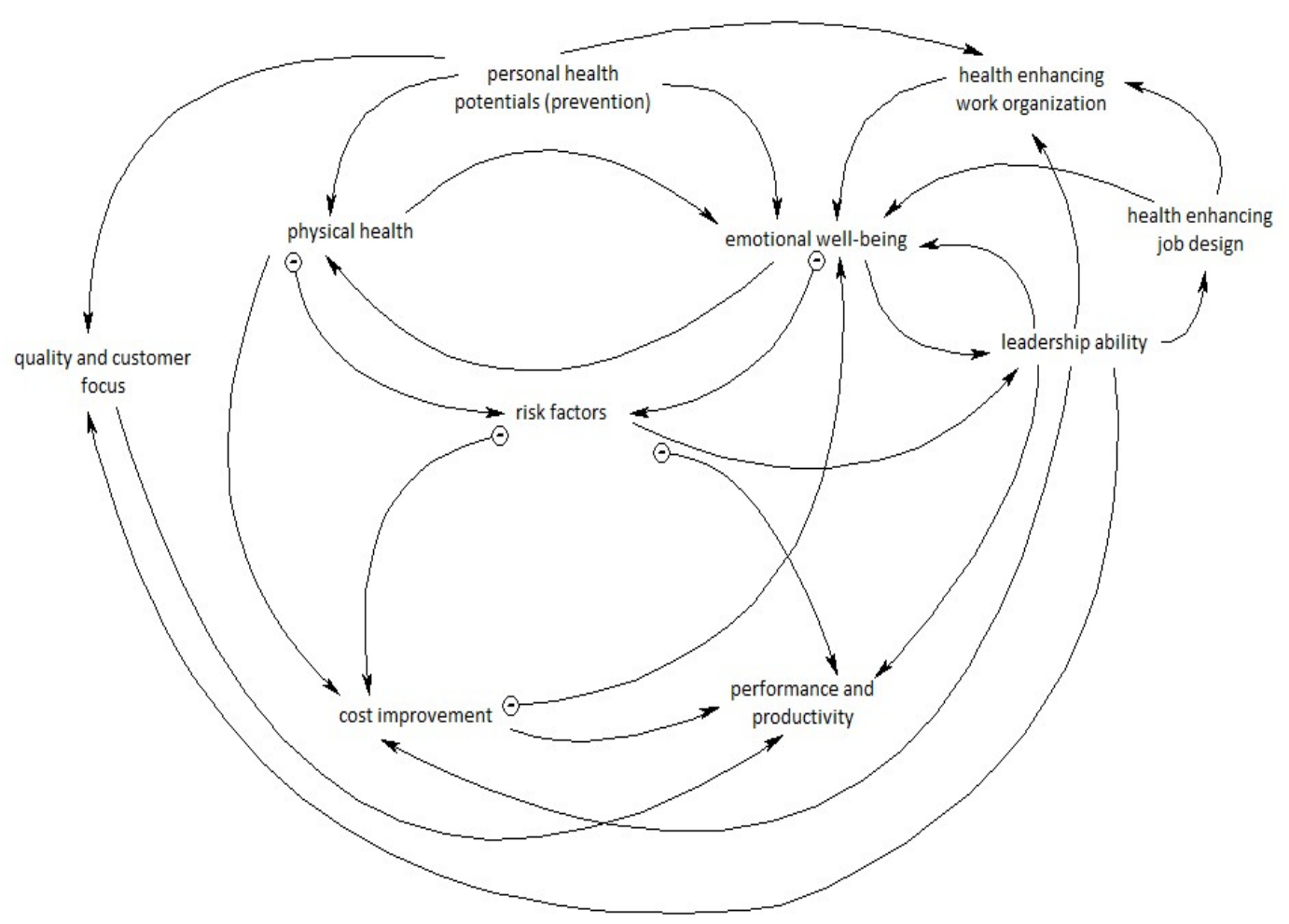




\section{Appendix 6: Documentation of the answers in the interview guide}

General practitioner A: The interview preparation documents were sent on 4 December 2017. The call took place on 8 December 2017 at $6.40 \mathrm{pm}$ to $7.35 \mathrm{pm}$. The answers were logged in the interview guide as follows:

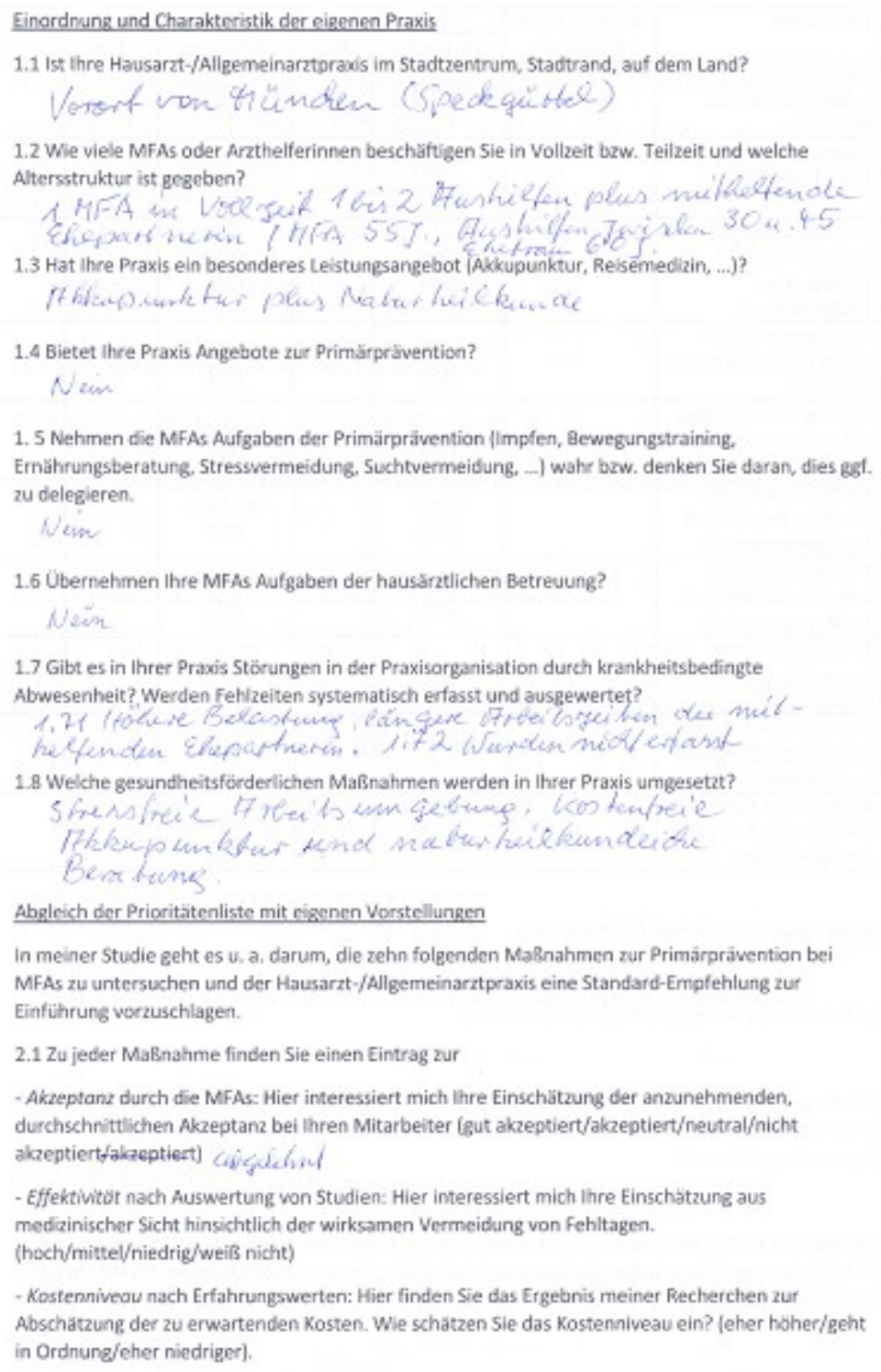

In meiner Studie geht es u. a. darum, die zehn folgenden Maßnahmen zur Primärpräwention bei MFAs zu untersuchen und der Hausarzt-/Algemeinaratpraxis eine Standard-Empfehlung zur Einführung vorzuschlagen.

$2.1 \mathrm{Zu} \mathrm{jeder} \mathrm{MaBnahme} \mathrm{finden} \mathrm{Sie} \mathrm{einen} \mathrm{Eintrag} \mathrm{zur}$

- Akzeptanz durch die MFAs: Hier interessiert mich lihre Einschätzung der anzunehmenden, durchschnittlichen Akzeptanz bei lihren Mitarbeiter Lgut akzeptiert/akzeptiert/neutral/nicht akreptiertfakaeptiert) cieguldial

- Effektivitöt nach Auswertung won Studien: Hier interessiert mich ithre Einschatzung aus mediainischer Sicht hinsichtlich der wirksamen Vermeidung von fehitager. (hoch/mittel/niedrig/weils nicht)

- Kostenniveau nach Erfahrungswerten: Hier finden Sie das Ergebnis meiner Recherchen zur Abschatzung der zu erwartenden Kosten. Wie schatzen Sie das Kostenniveav ein? (eher hoher/geht in Ordnung/eher niedriger]. 


\begin{tabular}{|c|c|c|c|c|c|c|}
\hline \multirow[t]{2}{*}{ Maßnahme } & \multirow{2}{*}{$\begin{array}{c}\text { angenommene } \\
\text { Akzeptanz }\end{array}$} & \multicolumn{2}{|c|}{ Effektivitat } & \multicolumn{2}{|c|}{ Kostenniveau } & \multirow[t]{2}{*}{ Prio. } \\
\hline & & Lt. Studie & Arzt & Schatzung & Arzt & \\
\hline $\begin{array}{l}\text { Übungen am } \\
\text { Arbeitsplatz (bis } 10 \\
\text { Minuten) }\end{array}$ & whorbia & $\begin{array}{c}\text { niedrig } \\
\text { (33) }\end{array}$ & movity & $\begin{array}{l}\text { niedrig } \\
(75)\end{array}$ & misitel & \\
\hline $\begin{array}{l}\text { Obungen für Daheim } \\
\text { (bis } 10 \text { Minuten) }\end{array}$ & cher thim: & $\begin{array}{c}\text { mittel } \\
(50)\end{array}$ & hoct & $\begin{array}{l}\text { seht niedrig } \\
\quad(100)\end{array}$ & medsy & \\
\hline $\begin{array}{l}\text { Geförderte Akthitat } \\
\text { nach der Arbeit } \\
\text { (Fitness, Yoga, } \\
\text { Laufgruppe, ...) }\end{array}$ & in & $\begin{array}{l}\text { mittel } \\
\text { (50) }\end{array}$ & $\Delta 4 x$ & $\begin{array}{c}\text { niedris } \\
{[7 S]}\end{array}$ & $\begin{array}{l}\text { ael } \\
\text { andel }\end{array}$ & \\
\hline $\begin{array}{l}\text { Training mit Schritt- } \\
\text { zahler (digital via Handy } \\
\text { oder Uhr) }\end{array}$ & hav: & $\begin{array}{l}\text { mittel } \\
{[66]}\end{array}$ & subs & $\begin{array}{c}\text { niedrig } \\
\text { (75) }\end{array}$ & $\sin 1 / 6$ & \\
\hline $\begin{array}{l}\text { Training in der Gruppe } \\
\text { mit Kolleginnen }\end{array}$ & $\omega$ & $\begin{array}{c}\text { sehr niedrig } \\
\text { (16) }\end{array}$ & wiolg & $\begin{array}{l}\text { mittel } \\
(62)\end{array}$ & muiter & \\
\hline $\begin{array}{l}\text { Gruppensitzung mit } \\
\text { externen Berater ( } 2 . \text { B. } \\
\text { Ernährungsberater) }\end{array}$ & & $\begin{array}{l}\text { mittel } \\
(42)\end{array}$ & heres on to & $\begin{array}{l}\text { sehr hoxh } \\
\text { (2S) }\end{array}$ & 100, & \\
\hline $\begin{array}{l}\text { Einzelgespräch mit } \\
\text { externen Berater (2. B. } \\
\text { Efnăhrungsberater) }\end{array}$ & eh & $\begin{array}{l}\text { mittel } \\
{[42]}\end{array}$ & merg & $\begin{array}{l}\text { sehr hoch } \\
\text { (25) }\end{array}$ & $k_{\phi}, C_{2}$ & \\
\hline $\begin{array}{l}\text { Einzelgesprach mit } \\
\text { einem Arzt (aus der } \\
\text { Praxis) }\end{array}$ & $e^{h}$ aen $f a$ & $\begin{array}{l}\text { mittel } \\
{[42]}\end{array}$ & $60 x_{2}^{2}$ & $\begin{array}{c}\text { mittel } \\
(37)\end{array}$ & $\sin \mathrm{Vac}$ & \\
\hline $\begin{array}{l}\text { Gesunde Snacks (z. B. } \\
\text { Obst) wom Arbeitgeber } \\
\text { in der Praxis }\end{array}$ & ehor ia & $\begin{array}{l}\text { mittel } \\
(58)\end{array}$ & miket & $\begin{array}{c}\text { mittel } \\
(50)\end{array}$ & meilla & \\
\hline $\begin{array}{l}\text { Regelmäßige Kontrolle } \\
\text { / Impfung (z. B. Grippe) } \\
\text { am Arbeitsplatz) }\end{array}$ & etw dean & $\begin{array}{l}\text { sehr hoch } \\
(100)\end{array}$ & 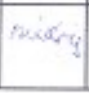 & $\begin{array}{l}\text { sehr niedrig } \\
{[100)}\end{array}$ & wedis & \\
\hline
\end{tabular}

Anmerkungen zu einzelnen Maßnahmen

2.2 Fehlen nach threm Dafürhalten Maßnahmen?

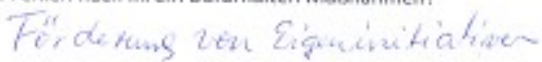
Lif 2.3 Whe worden Sie bei einer Entscheidung aber die Maßsnahmen die Kriterien Akzeptanz, Effektivität Entscheidungskriterien?

2.4 Wenn Sie Maßnahmen zum Gesundheitsmanagement einführen, würden Sie die Akzeptanz durch die MFAs erfragen? (bzw, haben Sie dies getan, falls Maßnahmen eingefüht wurden?) Falls eher nein, Grùnde datür?

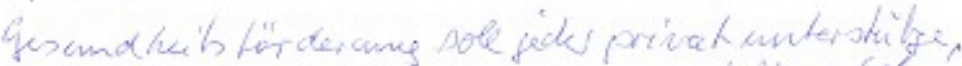

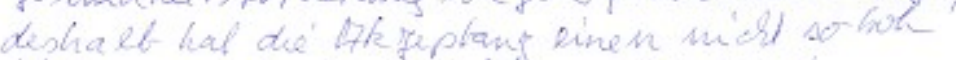

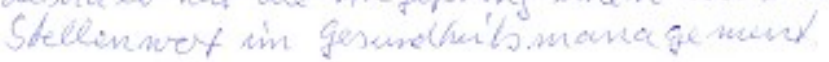


2.5 Die Ausarbeitung eirt's praxisspezifischen Einführungskonzepts erfordert Zeit. Wie viel Stunden würden Sie dafur veranschlagen? - Bzw. welche Zeitersparnis ist durch die Nutzung der StandardEmpfehlung moglich?

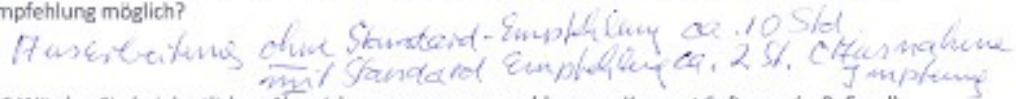

2.6 Wurden Sie bei deutlichen Abweichungen wom vorgeschlagenen Konzept Software (z. B. Excel) nutzen, um thre spezifische Entscheidung vorzubereiten?

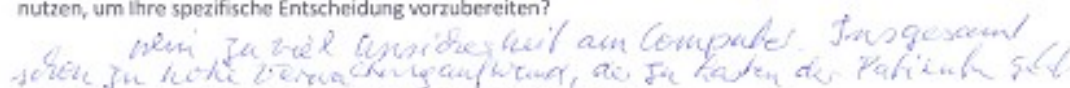

Einflussfaktoren zur Steverung der Produktivitatund der Performance

3.1 Welche Bedeutung messen Sie den folgenden Stellhebeln für die Reduzienung von krankheitsbedingten Fehltagen bei:

- Gesundheitsförderliche Maßnahmen für MFAs 3

- Arbeitsorpanisation der Praxis

- Stellenprofil / Job design der MFAs

- Führungsqualitàt/-kompetenz

3.2 Welche weiteren Ansatzpunkte und Maßnshmen für die Reduzierung von krankheitsbedingten Fehitagen sollten lhres Meinung nach für thre Praxis stärker bericksichtigt werden?

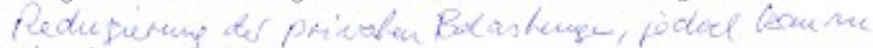
Leain fecos la

implementierung von Gesundheitsmansgement in der eigenen Praxis.

4,1 in welche Reihenfolge würden Sie die zuvor genannten vier Stellhebel angehen?

$$
\text { exlopedewa } 3,1
$$

4.2 Gibt es aus threr Sicht Gründe, warum gerade eine Arztpraxis im Vergleich zu anderen Organisationen und Unternehmen ein Gesundheitsmanagementsystem einfihren sollte?

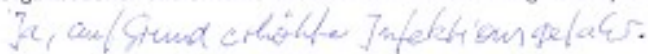

4.3 Wie praktizieren Sie bislang Maßnahmen zum Gesundheitsmanagement und welche Bedeutung messen Sie dem bei?

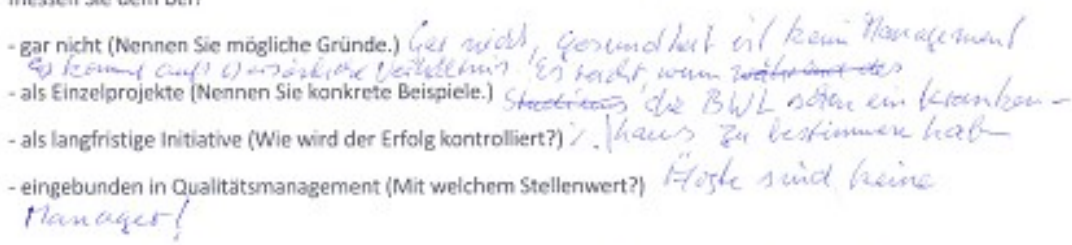

4.4 Weiche Bedeutung haben Konzepte wie „Gesunde Fuhrung", „Gesundheitskultur in der. Arztpraxis" oder „Gesundheitskompetenz MFA" (alle den Fortbildungsprogrammen von Ärstekammern entnommen) für Sie?

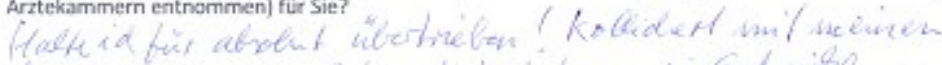

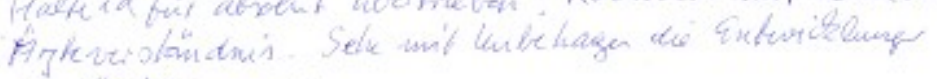

de rateleawnes 
General practitioner B: The interview preparation documents were sent on 4 December 2017. Because of the high workload in practice (flu outbreak), a telephone call was only possible during the holidays over Christmas. This took place on the 28th of December 2017 from 9.35am to 10.20am. The answers were first noted by the participating physician and then entered directly into the form via software:

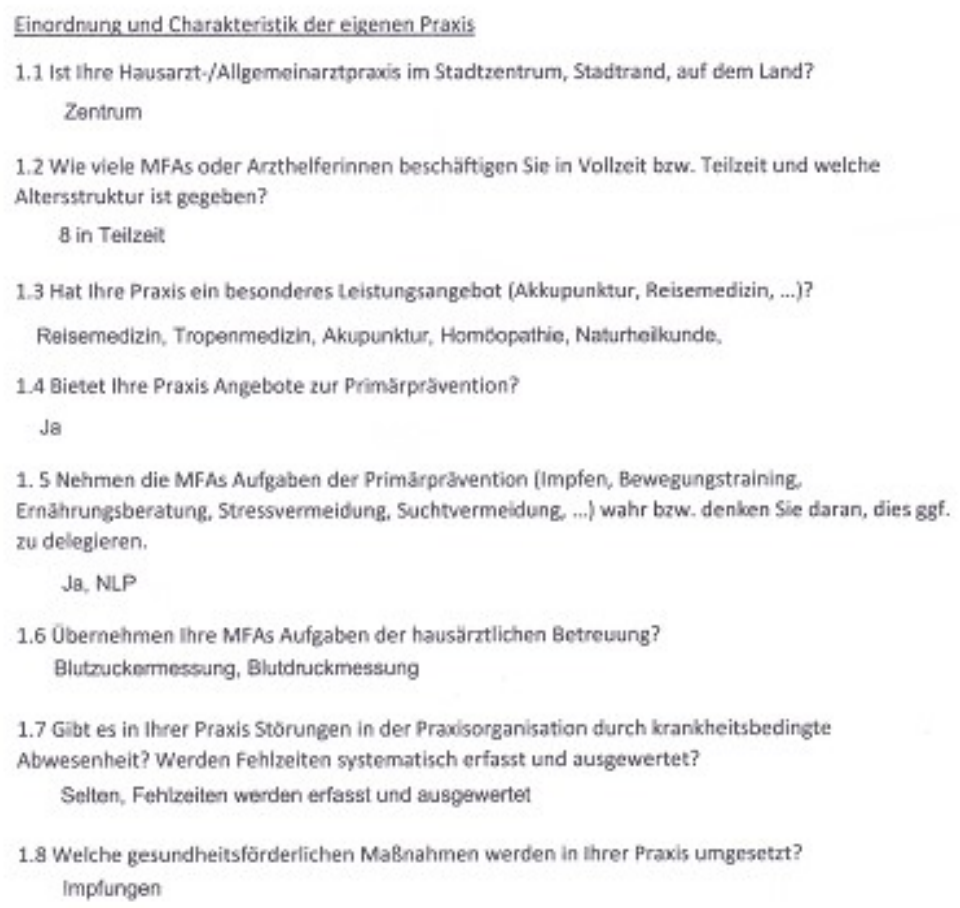

In meiner Studie geht es u. a. darum, die zehn folgenden Maßnahmen zur Primărpravention bel MFAs zu untersuchen und der Hausarzt-/Algemeinarztpraxis eine Standard-Empfehlung zur Einführung vorzuschlagen.

$2.1 \mathrm{Zu}$ jeder Maßnahme finden Sie einen Eintrag zur

- Akzeptanz durch die MFAs: Hier interessiert mich Ihre Einschatzung der anzunehmenden, durchschnittlichen Akzeptanz bei Ihren Mitarbeiter (gut akzeptiert/akzeptiert/neutral/nicht akzeptiert/gar nicht akzeptiert)

- Effektivitot nach Auswertung von Studien: Hier interessiert mich Ihre Einschătzung aus medizinischer Sicht hinsichtlich der wirksamen Vermeidung von Fehitagen. (hoch/mittel/niedrig/weis nicht]

- Kostennweau nach Erfahrungswerten: Hier finden Sie das Ergebnis meiner Recherchen zur Abschatzung der zu erwartenden Kosten. Wie schätzen Sie das Kostenniveau ein? leher hơher/geht in Ordnung/eher niedrigerl. 


\begin{tabular}{|c|c|c|c|c|c|c|}
\hline \multirow[t]{2}{*}{ Maßnahme } & \multirow{2}{*}{\begin{tabular}{|c|}
$\begin{array}{c}\text { angenommene } \\
\text { Akzeptanz }\end{array}$ \\
\end{tabular}} & \multicolumn{2}{|c|}{ Effektivität } & \multicolumn{2}{|c|}{ Kostenniveau } & \multirow[t]{2}{*}{ Prio. } \\
\hline & & Lt. Studie & Arst & Schätzung & Arzt & \\
\hline $\begin{array}{l}\text { Ubungen am } \\
\text { Arbeitsplatz (bis } 10 \\
\text { Minuten) }\end{array}$ & neutral & $\begin{array}{c}\text { niedrig } \\
{[33]}\end{array}$ & niedrig & $\begin{array}{c}\text { niedrig } \\
\text { (75) }\end{array}$ & niedrig & \\
\hline $\begin{array}{l}\text { Öbungen für Daheim } \\
\text { (bis } 10 \text { Minuten) }\end{array}$ & nicht akzepliert & $\begin{array}{l}\text { mittel } \\
(50)\end{array}$ & mittel & $\begin{array}{l}\text { sehr niedrie } \\
\qquad 100\}\end{array}$ & $\begin{array}{l}\text { sehr } \\
\text { niedrig }\end{array}$ & \\
\hline $\begin{array}{l}\text { Geförderte Aktivität } \\
\text { nach der Arbeit } \\
\text { (Fitness, Yoga, } \\
\text { Laufgruppe, ...) }\end{array}$ & akzeptiert & $\begin{array}{l}\text { mittel } \\
(\varsigma 0\rangle\end{array}$ & hech & $\begin{array}{c}\text { niedrig } \\
(75)\end{array}$ & mittel & \\
\hline $\begin{array}{l}\text { Training mit Schritt- } \\
\text { zăhler (digital via Handy } \\
\text { oder Uhr) }\end{array}$ & akzeptiert & $\begin{array}{c}\text { mittel } \\
(66)\end{array}$ & mittel & $\begin{array}{l}\text { niedrig } \\
\text { (75) }\end{array}$ & niedrig & \\
\hline $\begin{array}{l}\text { Training in der Gruppe } \\
\text { mit Kolleginnen }\end{array}$ & neutral & $\begin{array}{l}\text { sehr niedrig } \\
\qquad[16]\end{array}$ & niedrig & $\begin{array}{c}\text { mittel } \\
\langle 62\rangle\end{array}$ & mittel & \\
\hline $\begin{array}{l}\text { Gruppensitzung mit } \\
\text { externen Berater }[z, 8 . \\
\text { Ernahrungsberater }]\end{array}$ & nicht akzeptiert & $\begin{array}{l}\text { mittel } \\
{[42]}\end{array}$ & mittel & $\begin{array}{l}\text { seht hoch } \\
\text { (25) }\end{array}$ & sehr hoch & \\
\hline $\begin{array}{l}\text { Einzelzesprach mit } \\
\text { externen Berater ( } z \text {. B. } \\
\text { Ernahhrungsberater) }\end{array}$ & akzeptiert & $\begin{array}{l}\text { mittel } \\
(42)\end{array}$ & mittel & $\begin{array}{l}\text { sehr hoch } \\
\text { [25] }\end{array}$ & sehr hoch & \\
\hline $\begin{array}{l}\text { Einzeleesprach mit } \\
\text { einem Arzt (aus der } \\
\text { Praxis) }\end{array}$ & neutral & $\begin{array}{l}\text { mittel } \\
(42)\end{array}$ & mittel & $\begin{array}{l}\text { mittel } \\
(37)\end{array}$ & mittel & \\
\hline $\begin{array}{l}\text { Gesunde Snacks (z. B. } \\
\text { Obst) vom Arbeitgeber } \\
\text { in der Praxis }\end{array}$ & gut akzeptiert & $\begin{array}{l}\text { mittel } \\
\text { (58) }\end{array}$ & mittel & $\begin{array}{l}\text { mittel } \\
\text { (50) }\end{array}$ & hoch & \\
\hline $\begin{array}{l}\text { Rogelmäige Kontrolle } \\
\text { / Impfung (2. B. Grippe) } \\
\text { am Arbeitsplatz) }\end{array}$ & akzeptiert & $\begin{array}{l}\text { sehr hoch } \\
(100)\end{array}$ & $\begin{array}{l}\text { sehr } \\
\text { hoch }\end{array}$ & $\begin{array}{l}\text { sehr niedrig } \\
\text { (100) }\end{array}$ & niednig & \\
\hline
\end{tabular}

Anmerkungen zu einzelnen MaBnahmen

2.2 Fehlen nach lhrem Dafïrhalten Maßnahmen?

zuckerfreie Getranke am Arbeitsplatz

2.3 Wre würden Sie bei einer Entscheidung über die Maß̂nahmen die Kriterien Akzeptanz, Effektivităt und Kosten gewichten? (Angenommen Sie haben 100 Punkte zu wergeben.) Fehlen Entscheidungskriterien?

$$
40,40,20
$$

2.4 Wenn Sie Maßnahmen zum Gesundheitsmanagement einfuhren, würden Sie die Akzeptanz durch die MFAs erfragen? (baw. haben Sie dies getan, falls Maßnahmen eingefohrt wurden?) Falls eher nein, Gründe dafur?

Ja 
2.5 Die Ausarbeitung eines praxisspezifischen Einführungskonzepts erfordert Zelt. Wie viele Stunden würden Sie dafür veranschlagen? - Bzw. welche Zeitersparnis ist durch die Nutzung der StandardEmpfehlung mõglich?

$$
1 \text { Arbeitstag }
$$

2.6 Würden Sie bei deutlichen Abweichungen vom vorgeschlagenen Konzept Software ( 2 . B. Excel) nutzen, um thre spezifische Entscheidung vorzubereiten?

Einflussfaktoren zur Steuerung der Produktivităt und der Performance

3.1 Welche Bedeutung messen Sie den folgenden Stellhebeln fü die Reduzierung von krankheitsbedingten Fehltagen bei:

- Gesundheitsförderliche Maßnahmen für MFAs niedrig 4

- Arbeitsorganisation der Praxis hoch 2

-Stellenprofil / Job design der MFAs mittel 1

- Führungsqualitat/-kompetens hoch 3

3.2 Welche weiteren Ansatzpunkte und Maßnahmen fûr die Reduzierung von krankheitsbedingten Fehitagen soliten threr Meinung nach für thre Praxis stărker berücksichtigt werden?

Gutes Arbeitsklima, actaquate psychische und physische Belastung

Implementierung von Gesundheitsmanagement in der eigenen Praxis

4.1 In welcher Reihenfolge würden Sle die zuvor genannten vier Stellhebel angehen?

4.2 Gibt es aus threr Sicht Grônde, warum gerade eine Arztpraxis im Vergleich zu anderen Organisationen und Unternehmen ein Gesundheitsmanagementsystem einfuhren solite? Ein Unternehmen, dass mit der Gesundheit won Menschen arbeitet, sollie dies auch durch gesunde Mitarbehier verkorpern

4.3 Wie praktizieren Sie bislang Ma\&nahmen zum Gesundheitsmanagement und welche Bedeutung messen sie dem bei?

- gar nicht (Nennen Sie mógliche Grunde.)

- als Einzelprojekte [Nennen Sie konkrete Beispiele.) Grippeimpfung

- als langfristige initiative (Wie wird der Effolg kontrolliert?) Durchimplungsrate

- eingebunden in Qualitatsmanagement (Mit welchem Stellonwert?]

4.4 Welche Bedeutung haben Konzepte wie ,Gesunde Fuhrung", „Gesundheitskultur in der Arztpraxis" oder „Gesundheitskompetenz MFA" falle den fortbildungsprogrammen von Ärztekammern entnommen] für Sie?

Mittlere Bedeutung fơr den Erfolg der Praxis 
General practitioner C: The interview preparation documents were sent on 4 December 2017. After a telephone consultation, the doctor decided to answer the questions in peace, for a reply by fax. This was sent on the 11 th of December. Two open questions were sent by e-mail and answered on 20 and 27 December respectively. For reasons of anonymity, the following documents overwrite personal files in grey:

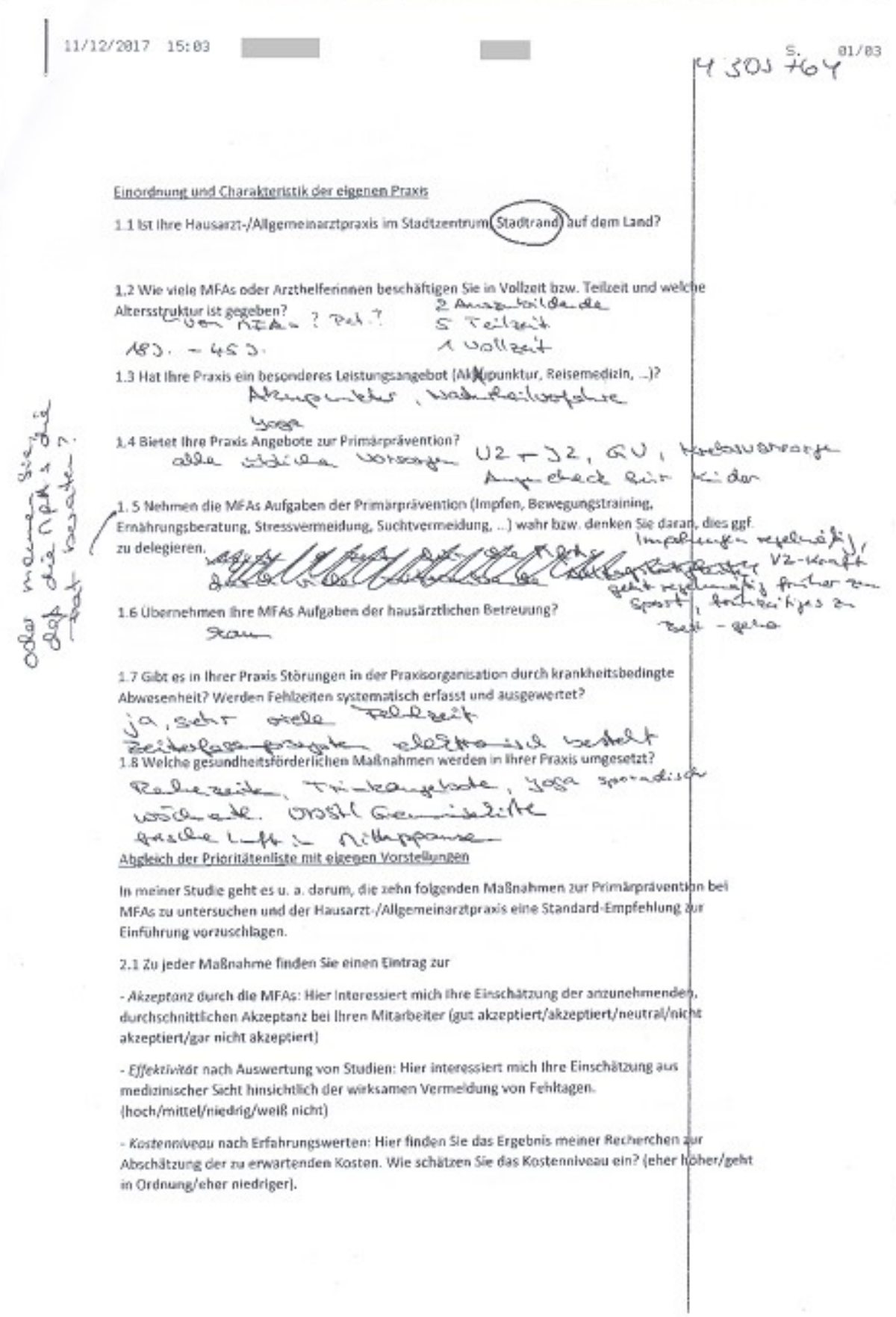


Jartingea? a $\theta$ ?

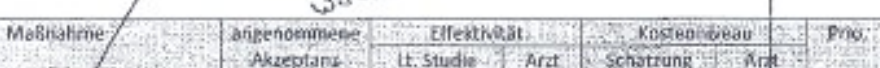

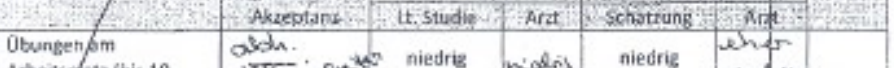

Arbeispotatz

Arbentspatz (bis 10 vo-jogen niedrie

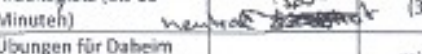

(bis 10 Minuten)

Geforderte Aktivitit

nach der Arbent

(Fisness, Yogs,

Loufgruppe, - )

zahler (digital via Hand

oder uhrs

Training in der Gruppe

mit Kolleginnen

Gruppensitaung mit

externen Berater $(2$. . B.

Emshrungsberater

Einzeisespróch mit

externen Berater /2. B.

Emahrungsberaterl

Einzeigesprasch mit

einem Arzt (aus der

Pravis)

Gesuede Snacks iz. B.

Obst) wom Arbengeter

in der Pravis

Regelmsikige Kontrolke

/ Inpfung (2. B. Grippe)

am Arbeitsplptz|

vermal

mittel

- 175

(75) wiedory

niedris

[100]

bermbited mittel witel niedrig wiedri

Anmerkungen wo eintelnen MaEnahsmen

2.2 Fehlen nach ihrem Dafurehalten Maß̈nahment?

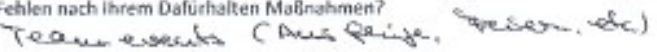

2.3 Wie wurden Sie bei einer Entscheidung uber die Maisnahmen die K.riterien Akzeptanz, Effteksivitat

und Kosten gewichten? (Angenommen Sie haben 100 Punkte zu vergeben.) Fehlen

Entscheidungsknterien?

2.4 Wenn Sie Mas̆nahmen zum Gesundheitsmanagement einfüren, wurden Sie die Akzeplesnz durch

die MFAs erfragen? (brw. haben Sie dies getan, falls Malnohmen eingetuhet wurden?) Falps eher

nein, Gründe dafur?

$$
\text { ja. wit Ptang vapelumsing }
$$




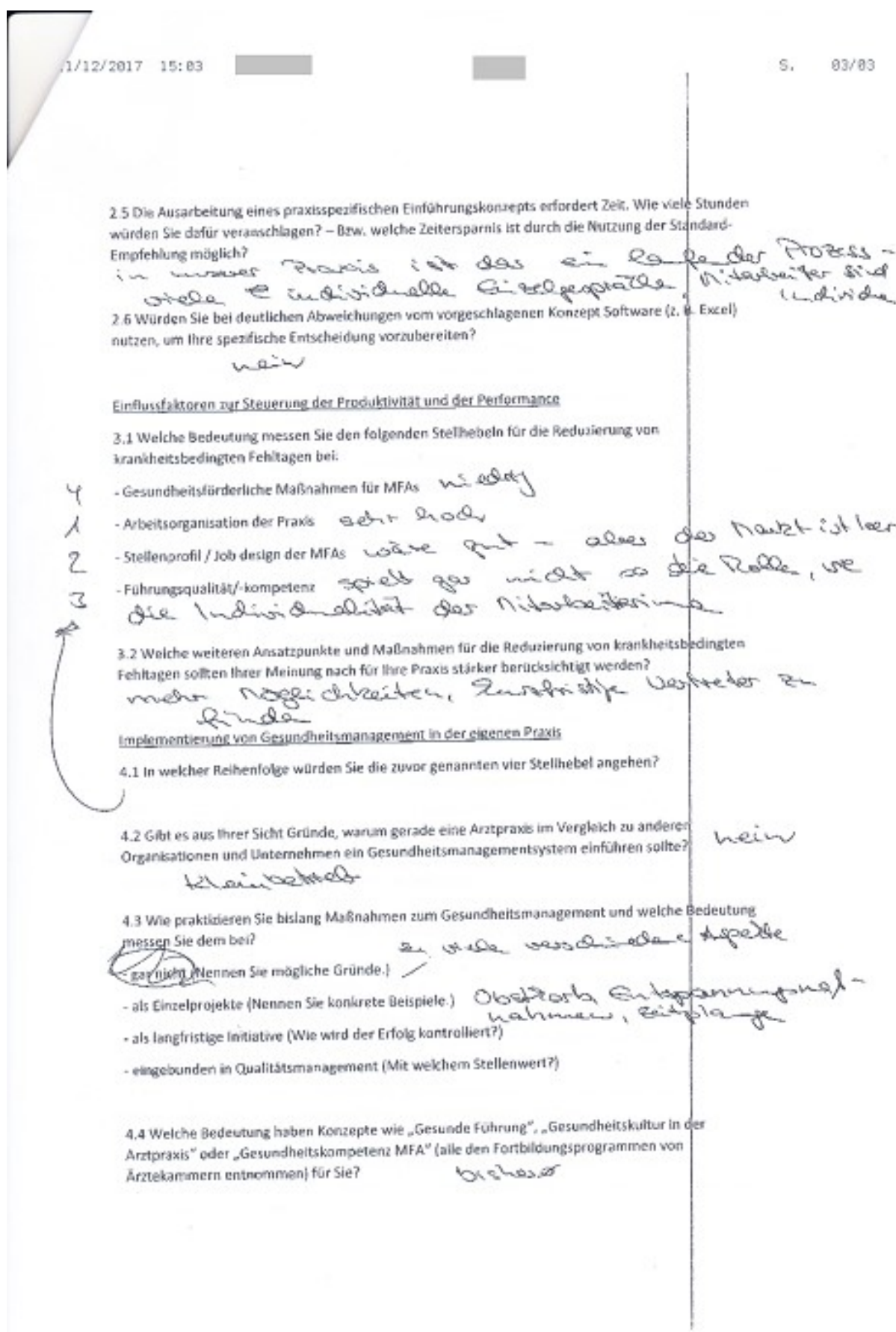


Von:

Cedric Ballin

Gesendet: Montag, 25. Dezember 2017 18:22

An:

Betreft: Fwd: AW: Gesundheitsmanagement / Entschuldigung

\begin{abstract}
Von:
Datum: 20, Dezember 2017 um 19:37:03 ME7

An: "

Betreff: AW: Gesundheitsmanagement / Entschuldigung

Lieber Herr Ballin.

schòn, dass ich Sie ein wenig unterstützen kann.

Zu 1.5. ist das bei uns nicht standardisiert, Die MFAs besprechen vieles während der

Routineabläufe. Da wird zu Sport geraten, ober gesunde Ernährung gesprochen, zu Yoga etc. getaten - erzählt, dass wir pel. auch furs Team Yoga machen. Aber das ist genauso wie meine Sprechstunde sehr individuell. Wir achten sehr darauf, was fur wen wie und wann passt. Einer gehgeschwächten alteren Dame wird etwas anderes geraten, als einen 22 jahhrigen jungen Mann

So wie es nicht, „das Entspannungsverfahren für jeden gibt, ist es erforderlich, den Einzelnen da abzuhalen, wo er ist. Und so kann das für einen Patienten das Zeitunglesen oder Sudakospielen sein, wahrend für einen anderen, der Spaziergang, der Kirchenchor, die Meditation, das Autogene Training zur Stressvermeidung dient, ich hatte schon MFAs, die sich da sehr engagjert haben. Eine Mitarbeiterin hat eine Ausbildung zum metabolic balancing gemacht und regelmãssig Einzelgespräche und Kurse angeboten. Derzeit bietet eine ehemaligu Mitarbeiterin am WE Kurse zur ausgewogenen Ernăhrung an. Wir haben Kurse zu Wickeln und Auflagen angeboten.

Eine Patientin bietet ab Januar $3 x$ wdchentlich Yoga in unseren Räumen an.

ich lasse das i.d.R. won den Mitarbeiterinnen kommen. Wenn sich eine MFA dafü interessiert, wird sie sehr unterstützt, aber zusätzlich möchte ich keiner Mitarbeiterin zusătzlich zu unserem .Altagswahnsinn" Zusatzaufgaben und -belastungen geben. Ich bin fast stols, keine, igelPravis" zu haben, wo Patienten Angst haben mussen, dass ihnen ständig etwas verkauft wird, wo sie unter Zugzwang sind. Dafur gibt es genug Angebote ausserhalb des vertrauten A.rztPatienten-Raumes. Individuelle geratung ist etwas anderes, was ich als Grundaufpabe eines Hausarztes sehe.

Zu 2.3. das ist wirklich eine frage, die ich mir (s.o.) nicht so oft stelle, weil ich mich ohen als Arzt verstehe und nicht als verkăufer. Aber vielleicht habe ich die Frage auch nicht exakt verstanden.
\end{abstract}

Schönen Abend

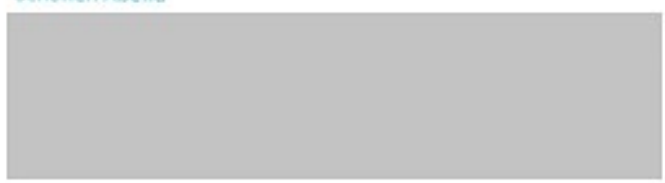

27.12 .2017 
Betreff: WG: Gesundheitsmanagement / Entschuidigung

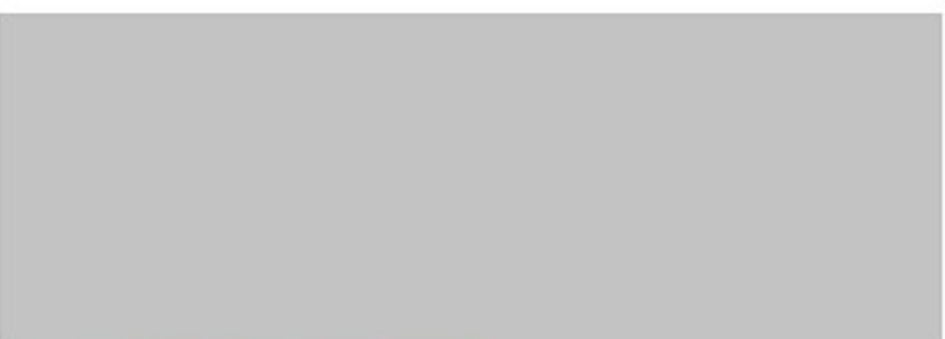

Zwei kleine Punkte waren noch von Interesse:

15. Nehmen die MFAs Aufgaben der Primärorävention (Impfen, Eewequngstraining Emàhrungsberatung. Stress-, Suchtvermeidung, ...) wahr bzw. denken Sie daran, dies ggf. zu delegieren?

Bei dieser Frage hatten Sie als Randbemerkung: ,Oder meinen Sie, dass die MFAs die Patienten beraten?" - Meine Frage ging tatsachlich in diese Richtung: denn lt, einer-

Studie denken ca. $80 \%$ der Allgemeinäzte daran, diese Aufgaben zu delegieren

2.3 Wie würden Sie bei einer Entscheidung über die Maßnahmen die Kriterien Akzeptanz, Effektivitat und Kosten gewichten? Fehlen Entscheidungskniterien?

Diese Frage ist offen geblieben. Da meine Arbeit sich mit dem Entscheidungsverhalten von Äzten (nicht bei Diagnosen und Therapien, da treffen sie ja standig

Entscheidungen) hefacst ware es wichtig zu wissen, welchem Kriterium Sie besondere

Bedeutung beimessen oder sehen Sie alle crei Kniterien als eher gleich wichtig an?

Oder wird eine Entscheidung gar nicht bewusst nach bestimmten Kriterien getroffen (Zu meinem Grundverstandnis: Entscheiden ist die Auswahl bewerteter Alternativen. Mir geht es $u$. a. darum herauszutinden, wie diese Bewertung erfolgt.

Ich würde mich freuen, zu diesen beiden Punkten noch lhre Sichtweise zu erfahree

Herzliche Graße

Cedric Ballin 
General practitioner D: The interview preparation documents were sent on 4 December 2017. The attending physician entered his answers during the phone call on 20 December 2017 from $1 \mathrm{pm}$ to $1.55 \mathrm{pm}$ and faxed them the following day.

Finordnungund Charakteristik der eigenen Praxis

1.1 Ist Ihre Hausarzt-/Aligemeinarztpraxis im Stadtzentrum, Stadtrand, auf dem Land?

1.2 Wie viele MFAs oder Arzthelferinnen beschăftigen Sie in Vollzeit brw. Teilzeit und weiche Altersstruktur ist gegeben?

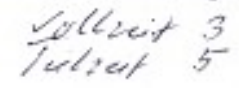

1.3 Hat Ihre Prawis ein besonderes Leistungsangebot (Akkupunktur, Reisemedizin, ....)?

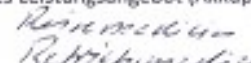

1.4 Bietet Ihre Prawis Angebote zur Primärprảvention?

$$
14
$$

1. 5 Nehmen die MFAs Aufgaben der Primärprävention (Implen, Bewegungstraining.

Ernährungsberatung, Stressvermeidung, Suchtvermeidung, ...) wahr bzw. denken Sie daran, dies gef.

zu delegieren.

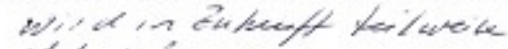

$$
\begin{aligned}
& \text { celpiot }
\end{aligned}
$$

1.6 Obernehmen Ihre MFAs Aufgaben der hausarztlichen Betreuung?

$$
\text { nim }
$$

1.7 Gibt es in Ihrer Praxis Störungen in der Praxisorganisation durch krankheitsbedingte Abwesenheit? Werden Fehlzeiten systematisch erfasst und ausgewertet?

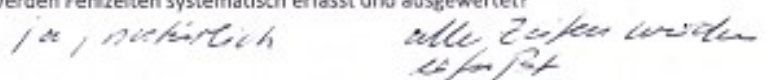

1.8 Welche gesundheitsförderlichen Maßnnahmen werden in Ihrer Praxis umgesetzt?

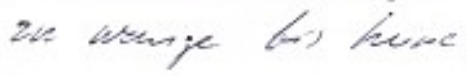

\section{Abgleich der Prioritatenliste mit eizenen Worstellungen}

In meiner Studie geht es u. a. darum, die zehn folgenden Maßnahmen zur Primărprãvention bei MFAs zu untersuchen und der Hausarzt-/Allgemeinarztpraxis eine Standard-Emptehlung zur Einführung vorzuschlagen.

$2.12 u$ jeder Maßnahme finden Sie einen Eintrag zur

- Akzeptanz durch die MFAs: Hier interessiert mich Ihre Einschätzung der anzunehmenden, durchschnittlichen Akzeptanz bei Ihren Mitarbeiter (gut akzeptiert/akzeptiert/neutral/nicht akzeptiert/gar nicht akzeptiert)

- Effektivität nach Auswertung von Studien: Hier interessiert mich Ihre Einschätzung aus medizinischer Sicht hinsichtlich der wirksamen Vermeidung wan Fehltagen. [hoch/mittel/niedrig/weiB nicht]

- Kostenniveau nach Erfahrungswerten: Hier finden Sie das Ergebnis meiner Recherchen zur Abschătzung der zu erwartenden Kosten. Wie schatzen Sle das Kostenniveau cin? (cher höher/geht in Ordnung/eher niedriger). 


\begin{tabular}{|c|c|c|c|c|c|c|}
\hline \multirow{2}{*}{ Maßnahme } & \multirow{2}{*}{\begin{tabular}{|c|} 
angenommene \\
Akzeptanz
\end{tabular}} & \multicolumn{2}{|c|}{ Effektivitat } & \multicolumn{2}{|c|}{ Kostenniveau } & \multirow[t]{2}{*}{ Prio. } \\
\hline & & Lt. Studie & Arrt & Schätzung & Arzt & \\
\hline $\begin{array}{l}\text { Arbeitsplatz (bis } 10 \\
\text { Minuten) }\end{array}$ & gor mont & $\begin{array}{c}\text { niedrig } \\
\text { (33) }\end{array}$ & Nuas & $\begin{array}{l}\text { niedrig } \\
\text { (75) }\end{array}$ & $\theta$ & 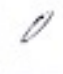 \\
\hline $\begin{array}{l}\text { Obungen fur Daheim } \\
\text { (bis } 10 \text { Minuten) }\end{array}$ & & $\begin{array}{c}\text { mittel } \\
{[50]}\end{array}$ & $\begin{array}{l}\text { werthe } \\
\text { jest }\end{array}$ & $\begin{array}{c}\text { sehr niedrig } \\
(100)\end{array}$ & phas & $/$ \\
\hline $\begin{array}{l}\text { Geforderte Aktivitat } \\
\text { nach der Arbeit } \\
\text { (Fitness, Yoga, } \\
\text { Lautgrupge, ...) }\end{array}$ & 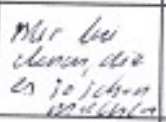 & $\begin{array}{l}\text { mittel } \\
(50)\end{array}$ & Borh & $\begin{array}{l}\text { niedrig } \\
\text { (75) }\end{array}$ & cathel & 2 \\
\hline $\begin{array}{l}\text { Training mit Schritt- } \\
\text { zahler (digital via Handy } \\
\text { oder Uhr) }\end{array}$ & the & $\begin{array}{c}\text { mittel } \\
(66)\end{array}$ & / & $\begin{array}{l}\text { niedrig } \\
(75)\end{array}$ & $/ 2$ & 2 \\
\hline $\begin{array}{l}\text { Training in der Gruppe } \\
\text { mit Kolleginnen }\end{array}$ & & $\begin{array}{c}\text { sehr niedrig } \\
\text { (16) }\end{array}$ & fork & $\begin{array}{c}\text { mittel } \\
(62)\end{array}$ & wetted & L \\
\hline $\begin{array}{l}\text { Gruppensitzung mit } \\
\text { externen Berater (z. B. } \\
\text { Emahrungsberater) }\end{array}$ & No & $\begin{array}{c}\text { mittel } \\
(42)\end{array}$ & $k+4 / n$ & $\begin{array}{l}\text { sehr hoch } \\
\text { (25) }\end{array}$ & nental & 2 \\
\hline $\begin{array}{l}\text { Einzelgespräch mit } \\
\text { externen Berater (2. B. } \\
\text { Ernshihrungsberater) }\end{array}$ & & $\begin{array}{l}\text { mittel } \\
(42)\end{array}$ & $x=2 y$ & $\begin{array}{l}\text { sehr hoch } \\
\text { [25] }\end{array}$ & 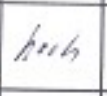 & \\
\hline $\begin{array}{l}\text { Einzelgesprăch mit } \\
\text { einem Arzt (aus der } \\
\text { Praxis) }\end{array}$ & & $\begin{array}{l}\text { mittel } \\
\text { (42) }\end{array}$ & KOAD & $\begin{array}{c}\text { mittel } \\
(37)\end{array}$ & wall & / \\
\hline $\begin{array}{l}\text { Gesunde Snacks }[2,8, \\
\text { OOst) vom Arbeitgeber } \\
\text { in der Praxis }\end{array}$ & hooh & $\begin{array}{l}\text { mittel } \\
\text { (58) }\end{array}$ & mall & $\begin{array}{l}\text { mittel } \\
\text { (50) }\end{array}$ & eothis & $/$ \\
\hline $\begin{array}{l}\text { RegelmaSige Kontrolle } \\
\text { /Impfung (z. B. Grippe) } \\
\text { am Arbeitsplatz) }\end{array}$ & neigel & $\begin{array}{l}\text { sehr hoch } \\
(100)\end{array}$ & kith & $\begin{array}{c}\text { sehr niedrig } \\
(100)\end{array}$ & 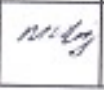 & I \\
\hline
\end{tabular}

Anmerkungen zu einzelnen Maßnahmen

2.2 Fehlen nach lhrem Dafürhalten Maßnahmen?

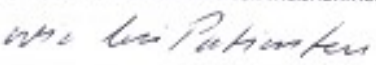

$$
\text { copelonis/se Lect ups }
$$

2.3 Wie würden Sie bei einer Entscheidung über die Maßnahmen die Kriterien Akzeptanz, Effektivität und Kosten gewichten? (Angenommen Sle haben 100 Punkte zu vergeben.) Fehlen. Entscheidungskriterien?

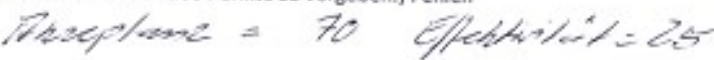

$$
\begin{aligned}
& \lim _{x \rightarrow t_{x \rightarrow 3}}=5
\end{aligned}
$$

2.4 Wenn Sie Maßnahmen zurn Gesundheitsmanagement einführen, würden Sie dic Algeptanz durch die MFAs erfragen? (brw, haben Sie dies getan, falls Maßnahmen eingefuhrt wurden?) Falls eher nein, Gründe dafür?

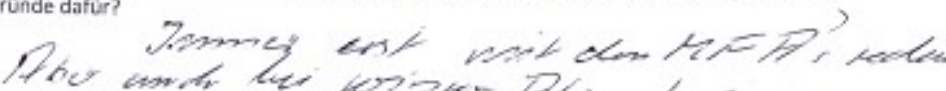

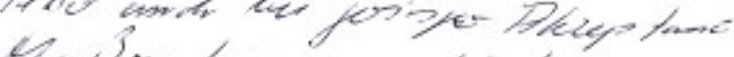

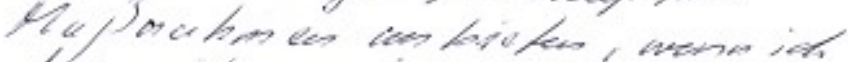

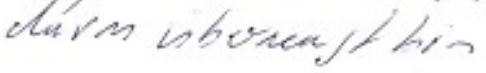


2.5 Die Ausarbeitung eines prawisspezifischen Einführungskonzepts erfordert Zeit. Wie viele Stunden würden Sie dafür veranschlagen? - Bzw. welche Zeitersparnis ist durch die Nutzung der StandardEmpfehlung möglich?

$$
\begin{aligned}
& 18.1 \%
\end{aligned}
$$

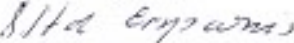

2.6 Würden Sie bei deutlichen Abweichungen vom vorgeschlagenen Konzept Software (z. B. Exce) nutzen, um thre spezifische Entscheidung vorzubereiten?

$$
\text { lix } \rightarrow
$$

Einflusstaktoren zur Steucrung der Produktivitat und der Performance

3.1 Welche Bedeutung messen Sie den folgenden Stellhebeln für die Redurierung von krankheitsbedingten Fehltagen bei:

- Gesundheitsförderliche Maßnahmen für MFAs

- Arbeitsorganisation der Praxis

$$
\begin{aligned}
& 948 \\
& \text { |lmint } \\
& \text { jers } \\
& \text { heck }
\end{aligned}
$$

- Stellenprafil / Job design der MFAs

- Führungsqualităt/-kompetenz

$$
\begin{aligned}
& \text { sel } k 004
\end{aligned}
$$

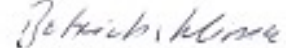

3.2 Welche weiteren Ansatzpunkte und Maßßnahmen für die Reduziefung von krankheitsbedingten Fehitagen sollten Ihrer Meinung nach für Ihre Praxis stärker berucksichtigt werden?

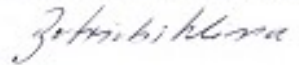

Imolementierung won Gesundheitsmanagement in der eigenen Praxis

4.1 In welcher Reihenfolge würden Sie die zuwor genannten vier Stellhebel angehen?

4.2 Gibt es aus Ihrer Sicht Gründe, warum gerade eine Arztpraxis im Vergleich zu anderen Organisationen und Unternehmen ein Gesundheitsmanagementsystem einfuhren sollte?

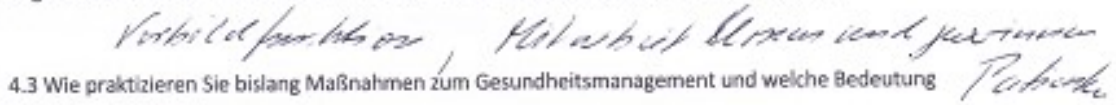
messen Sie dem bei?

- par nicht (Nennen Sie mõgliche Gründe.)

- als Einzelprojekte (Nennen Sie kenkrete Beisplele.)

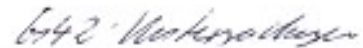

- als langfristige Initiative (Wie wird der Erfolg kontrolliert?)

- eingebunden in Qualitätsmanagement (Mit welchem Stellenwert?)

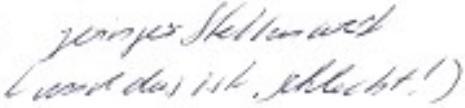

4.4 Welche Bedeutung haben Konzepte wir "Gesunde Fühnung", Gesundheitskultur in der Arztpravis" oder "Gesundheitskompetenz MFA* (alle den fortbildungsprogrammen won Ärztekammern entnommen) für Sie?

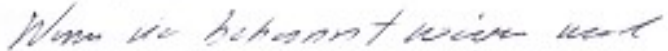

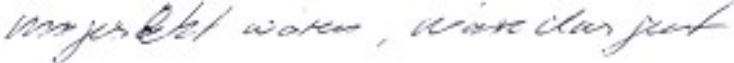




\section{Appendix 7: Data collection sheets for scoring results}

\begin{tabular}{|l|c|}
\hline \multirow{2}{*}{ Personal health promotion activities } & Acceptance \\
\cline { 2 - 2 } & \multicolumn{1}{|c|}{ Score } \\
\hline Programs for activity promotion & \\
\hline - Training at the workplace & \\
\hline - Training at home & \\
\hline - Sponsored activity after work & \\
\hline - Training with step counter or watch & \multicolumn{2}{|l|}{} \\
\hline - Training within a group of colleagues & \\
\hline Activities for weight reduction and change of nutrition & \multicolumn{2}{|l|}{} \\
\hline - Group session nutrition consulting (external) & \\
\hline - Single session nutrition consulting (external) & \\
\hline - Single session nutrition consulting (internal) & \multicolumn{2}{|l}{} \\
\hline - Healthy snacks/food at work & \\
\hline Vaccinating programs & \\
\hline - Vaccinating programs at work & \\
\hline - Vaccinating in private life & \\
\hline Further proposed measures & \\
\hline - Proposed in the MFA survey & - Proposed by the general practitioner \\
\hline
\end{tabular}

\begin{tabular}{|c|c|c|c|c|c|c|c|}
\hline \multirow{3}{*}{$\begin{array}{l}\text { Personal health } \\
\text { promotion activities }\end{array}$} & \multicolumn{7}{|c|}{ Effectivity } \\
\hline & \multicolumn{3}{|c|}{ Literature review (75\%) } & \multicolumn{3}{|c|}{ GP Interviews (25\%) } & \multirow[t]{2}{*}{ Total } \\
\hline & $\begin{array}{l}\text { Stu- } \\
\text { dies }\end{array}$ & $\begin{array}{c}\text { Level of } \\
\text { influence }\end{array}$ & Score & $\begin{array}{c}\text { Number } \\
\text { of GPs }\end{array}$ & $\begin{array}{l}\text { Rating } \\
\text { points }\end{array}$ & Score & \\
\hline \multicolumn{8}{|l|}{ Programs for activity promotion } \\
\hline \multicolumn{8}{|l|}{ - Training at the workplace } \\
\hline \multirow{2}{*}{\multicolumn{8}{|c|}{ - Training at home }} \\
\hline \multicolumn{2}{|l|}{ - Sponsored activity after work } & & & & & & \\
\hline \multicolumn{8}{|l|}{ - Training with step counter or watch } \\
\hline \multicolumn{8}{|c|}{\begin{tabular}{l|l} 
- Training within a & \\
group of colleagues &
\end{tabular}} \\
\hline \multicolumn{8}{|c|}{ Activities for weight reduction and change of nutrition } \\
\hline \multicolumn{8}{|l|}{$\begin{array}{l}\text { - Group session nutrition } \\
\text { consulting (external) }\end{array}$} \\
\hline \multicolumn{8}{|l|}{$\begin{array}{l}\text { - Single session nutrition } \\
\text { consulting (external) }\end{array}$} \\
\hline \multicolumn{8}{|l|}{$\begin{array}{l}\text { - Single session nutrition } \\
\text { consulting (internal) }\end{array}$} \\
\hline \multirow{2}{*}{\multicolumn{8}{|c|}{$\begin{array}{l}\text { - Healthy snacks/food at work } \\
\text { Vaccinating programs }\end{array}$}} \\
\hline & & & & & & & \\
\hline \multicolumn{8}{|l|}{ - Vaccinating programs at work } \\
\hline \multirow{2}{*}{\multicolumn{8}{|c|}{$\begin{array}{l}\text { - Vaccinating in private life } \\
\text { Further proposed measures }\end{array}$}} \\
\hline & & & & & & & \\
\hline \multicolumn{8}{|l|}{ - Proposed in the MFA survey } \\
\hline - Proposed by the general practitioner & & & & & & & \\
\hline
\end{tabular}




\begin{tabular}{|c|c|c|c|c|c|c|c|}
\hline \multirow{3}{*}{$\begin{array}{l}\text { Personal health promotion } \\
\text { activities }\end{array}$} & \multicolumn{7}{|c|}{ Cost level } \\
\hline & \multicolumn{4}{|c|}{$\begin{array}{c}\text { Experience and market situation } \\
(\mathbf{7 5 \% )}\end{array}$} & \multicolumn{2}{|c|}{$\begin{array}{l}\text { GP Interviews } \\
(25 \%)\end{array}$} & \multirow[t]{2}{*}{$\begin{array}{l}\text { Total } \\
\text { score }\end{array}$} \\
\hline & $\begin{array}{c}\text { Cost of } \\
\text { working } \\
\text { hours } \\
\end{array}$ & Score & $\begin{array}{l}\text { External } \\
\text { Costs }\end{array}$ & Score & $\begin{array}{c}\text { Rank- } \\
\text { ing }\end{array}$ & Score & \\
\hline \multicolumn{8}{|l|}{ Programs for activity promotion } \\
\hline \multicolumn{8}{|l|}{ - Training at the workplace } \\
\hline \multicolumn{8}{|l|}{ - Training at home } \\
\hline \multicolumn{8}{|l|}{ - Sponsored activity after work } \\
\hline \multicolumn{8}{|l|}{ - Training with step counter or watch } \\
\hline \multicolumn{8}{|l|}{ - Training within a group of colleagues } \\
\hline \multicolumn{8}{|c|}{ Activities for weight reduction and change of nutrition } \\
\hline \multicolumn{8}{|c|}{ - Group session nutrition consulting (external) } \\
\hline \multicolumn{8}{|c|}{ - Single session nutrition consulting (external) } \\
\hline \multicolumn{8}{|c|}{ - Single session nutrition consulting (internal) } \\
\hline \multicolumn{8}{|l|}{ - Healthy snacks/food at work } \\
\hline \multicolumn{8}{|l|}{ Vaccinating programs } \\
\hline \multicolumn{8}{|l|}{ - Vaccinating programs at work } \\
\hline \multicolumn{8}{|l|}{ - Vaccinating in private life } \\
\hline \multicolumn{8}{|l|}{ Further proposed measures } \\
\hline \multicolumn{8}{|l|}{ - Proposed in the MFA survey } \\
\hline - Proposed by the general practitioner & & & & & & & \\
\hline
\end{tabular}

